\title{
Photo-Induced Ortho-C-H Borylation of Arenes through In Situ Generation of Rhodium(II) Ate Complexes
}

Jin Tanaka, ${ }^{\dagger}$ Yuki Nagashima, ${ }^{*}+$ Antônio Junio Araujo Dias, and Ken Tanaka*,†

† Department of Chemical Science and Engineering, Tokyo Institute of Technology, O-okayama, Meguro-ku, Tokyo 1528550, Japan

\section{Table of Contents}

$\begin{array}{ll}\text { 1. General } & \text { S2 }\end{array}$

2. Rhodium-Catalyzed Photo-Induced C-H Borylation $\quad$ S3

3. Direct Various Elementalization Processes in One-Pot $\quad$ S17

$\begin{array}{ll}\text { 4. Control Experiments } & \text { S20 }\end{array}$

5. Synthesis of $\mathrm{Cp}^{\mathrm{A} 3} \mathrm{Rh}(\mathrm{cod})$ Complex and $\mathrm{Rh}(\mathrm{I})$ Catalyzed Experiments S21

6. UV-vis Absorption Study $\quad$ S22

$\begin{array}{ll}\text { 7. Computational Study } & \text { S24 }\end{array}$

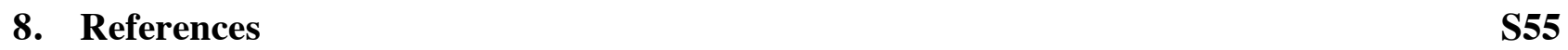

$\begin{array}{lr}\text { 9. NMR Spectra } & \text { S58 }\end{array}$ 


\section{General}

Materials: Anhydrous EtOH (No. 054-08705), THF (No. 200-00486), toluene (No. 202-18675), and 1,4-dioxane (No. 042-31655) were obtained from Wako and used as received. Anhydrous $\left(\mathrm{CH}_{2} \mathrm{Cl}\right)_{2}$ (No. 28,450-5), MeCN (No. 27,100-4), acetone (No. 27,072-5), and MeOH (No. 32,241-5) were obtained from Aldrich and used as received. Solvents for the synthesis of substrates were dried over Molecular Sieves $4 \AA$ (Wako) prior to use. Rh(III) complexes $\left[\mathrm{CpRhI}_{2}\right]_{\mathrm{n}},{ }^{1}\left[\mathrm{Cp}^{\mathrm{E} R h C l}\right]_{2},{ }^{2}$ $\left[\mathrm{Cp}^{\mathrm{A} 1} \mathrm{RhCl}_{2}\right]_{2},{ }^{3}\left[\mathrm{Cp}^{\mathrm{A} 2} \mathrm{RhCl}_{2}\right]_{2},{ }^{4}$ and $\left[\mathrm{Cp}^{\mathrm{A}} \mathrm{RhCl}_{2}\right]_{2},{ }^{3}$ arenes $\mathbf{1 f},{ }^{5} \mathbf{1 h},{ }^{6} \mathbf{1 l},{ }^{7} \mathbf{1 o},{ }^{8} \mathbf{1 q},{ }^{9} \mathbf{1 r},{ }^{10} \mathbf{1 s},{ }^{10} \mathbf{1 t},{ }^{11} \mathbf{1 v},{ }^{13}$ $\mathbf{1 x},{ }^{14} \mathbf{1 y},{ }^{14} \mathbf{1 z},{ }^{15}$ and $\mathbf{7}^{12}$ were already reported. All other reagents were obtained from commercial sources and used as received. Preparative thin-layer chromatography was performed with silica gel 60 from Merck.

Instrumentation: ${ }^{1} \mathrm{H},{ }^{13} \mathrm{C},{ }^{19} \mathrm{~F}$, and ${ }^{11} \mathrm{~B}$ NMR spectra were collected on a Bruker AVANCE III HD 400 spectrometer at ambient temperature. Chemical shifts are expressed in $\delta(\mathrm{ppm})$ values, and coupling constants are expressed in hertz $(\mathrm{Hz})$. The following abbreviations are used: $\mathrm{s}=$ singlet, $\mathrm{d}=$ doublet, $\mathrm{t}=$ triplet, $\mathrm{q}=$ quartet, $\mathrm{m}=$ multiplet, and $\mathrm{br}=$ broad. ESI mass spectra were measured on a Bruker micrOTOF Focus II spectrometer. EI mass spectra with gas chromatography were measured on an Agilent 5977B spectrometer. UV-vis absorption spectra were recorded on a JASCO V-630 spectrophotometer. Melting points were determined with a Mettler Toledo MP50 One Click Melting Point System and uncorrected. All reactions were carried out in oven-dried glassware with magnetic stirring. Photoreactions were performed in approximately $1 \mathrm{~cm}$ from a blue LED light (Kessil A160WE TUNA Blue). 


\section{Rhodium-Catalyzed Photo-Induced C-H Borylation}

Representative procedure for rhodium-catalyzed photo-induced ortho-borylation (3a, Scheme 1a): $\left[\mathrm{Cp}^{\mathrm{A} 3} \mathrm{RhCl}_{2}\right]_{2}(6.0 \mathrm{mg}, 0.0050 \mathrm{mmol}), \mathrm{NaO}{ }^{t} \mathrm{Bu}(1.9 \mathrm{mg}, 0.020 \mathrm{mmol})$ and $\mathrm{THF}(0.4 \mathrm{~mL})$ were placed in $3 \mathrm{~mL}$ screw cap vial and the mixture was stirred at room temperature for $10 \mathrm{~min}$. After stirring, to this mixture was added $2(101.6 \mathrm{mg}, 0.400 \mathrm{mmol}),\left[\operatorname{Ir}(\mathrm{dFppy})_{3}\right](1.5 \mathrm{mg}, 0.0020 \mathrm{mmol})$, $1 \mathbf{a}(26.4 \mu \mathrm{L}, 0.200 \mathrm{mmol})$ and THF $(1.6 \mathrm{~mL})$ in this order. The vial was capped and wrapped with a Teflon seal. The mixture was stirred at room temperature under Ar with blue LEDs irradiation for 18 $\mathrm{h}$. The resulting mixture was concentrated under reduced pressure. To the residue were added 1,3,5trimethylbenzene (as an internal standard) and $\mathrm{CDCl}_{3}$. Then NMR yield was analyzed by ${ }^{1} \mathrm{H} \mathrm{NMR}$.

\section{1-[2-(4,4,5,5-Tetramethyl-1,3,2-dioxaborolan-2-yl)phenyl]-1H-pyrazole (3a, Scheme 1a) ${ }^{16}$}<smiles>Brc1ccccc1-n1cccn1</smiles>

82\% NMR yield; ${ }^{1} \mathrm{H}$ NMR (400 MHz, $\left.\mathrm{CDCl}_{3}\right) \delta 7.87(\mathrm{~d}, J=2.1 \mathrm{~Hz}, 1 \mathrm{H}), 7.66(\mathrm{~d}, J=2.1 \mathrm{~Hz}, 1 \mathrm{H})$, $7.59(\mathrm{~d}, J=6.3 \mathrm{~Hz}, 1 \mathrm{H}), 7.44-7.37(\mathrm{~m}, 2 \mathrm{H}), 7.30(\mathrm{dd}, J=6.9,1.5 \mathrm{~Hz}, 1 \mathrm{H}), 6.45(\mathrm{dd}, J=2.1,2.1 \mathrm{~Hz}$, $1 \mathrm{H}), 1.36(\mathrm{~s}, 12 \mathrm{H})$.

Representative procedure for one-pot oxidation (4a, Scheme 1a): To the crude residue was added acetone $(5.0 \mathrm{~mL})$. Then, Oxone ${ }^{\circledR}(135.3 \mathrm{mg}, 0.400 \mathrm{mmol})$ in $1.0 \mathrm{~mL}$ of water was added dropwise. Upon the complete addition, the mixture was vigorously stirred at room temperature under air for $2 \mathrm{~h}$. Then saturated aqueous $\mathrm{Na}_{2} \mathrm{~S}_{2} \mathrm{O}_{3}(2.0 \mathrm{~mL})$ was added, and the aqueous layer was extracted with EtOAc. The combined organic layer was washed with brine, dried over $\mathrm{Na}_{2} \mathrm{SO}_{4}$, and concentrated. The residue was purified by preparative thin-layer chromatography (PTLC, $n$ hexane/dichloromethane $=1: 1)$ to give $\mathbf{4 a}(20.0 \mathrm{mg}, 0.125 \mathrm{mmol}, 62 \%$ yield $)$ as a colorless oil.

\section{2-(1H-Pyrazol-1-yl)phenol (4a, Scheme 1a $)^{8}$}

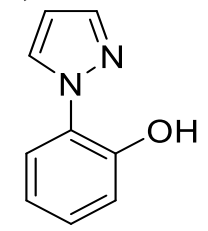

${ }^{1} \mathrm{H}$ NMR $\left(400 \mathrm{MHz}, \mathrm{CDCl}_{3}\right) \delta 11.37(\mathrm{~s}, 1 \mathrm{H}), 8.00(\mathrm{~d}, J=2.2 \mathrm{~Hz}, 1 \mathrm{H}), 7.72(\mathrm{~d}, J=2.2 \mathrm{~Hz}, 1 \mathrm{H})$, $7.37(\mathrm{dd}, J=7.9,1.5 \mathrm{~Hz}, 1 \mathrm{H}), 7.21-7.14(\mathrm{~m}, 1 \mathrm{H}), 7.10(\mathrm{dd}, J=7.9,1.5 \mathrm{~Hz}, 1 \mathrm{H}), 6.91(\mathrm{ddd}, J=7.9$, $7.9,1.5 \mathrm{~Hz}, 1 \mathrm{H}), 6.50(\mathrm{dd}, J=2.2,2.2 \mathrm{~Hz}, 1 \mathrm{H}) ;{ }^{13} \mathrm{C} \mathrm{NMR}\left(\mathrm{CDCl}_{3}, 100 \mathrm{MHz}\right) \delta 149.3,138.9,127.6$, $126.7,124.9,119.5,119.1,117.9,106.8$.

3,5-Dimethyl-1-[2-(4,4,5,5-tetramethyl-1,3,2-dioxaborolan-2-yl)phenyl]-1H-pyrazole $(3 \mathbf{b}$, Scheme 1a) ${ }^{16}$<smiles>Cc1cc(C)n(-c2ccccc2Br)n1</smiles>

95\% NMR yield, reaction time: $18 \mathrm{~h} ;{ }^{1} \mathrm{H}$ NMR $\left(400 \mathrm{MHz}, \mathrm{CDCl}_{3}\right) \delta 7.74(\mathrm{dd}, J=7.4,1.4 \mathrm{~Hz}, 1 \mathrm{H})$, 7.47 (ddd, $J=7.6,7.6,1.4 \mathrm{~Hz}, 1 \mathrm{H}), 7.37$ (ddd, $J=7.6,7.4,1.4 \mathrm{~Hz}, 1 \mathrm{H}), 7.29-7,24$ (m, 1H), 5.93 (s, 1H), 2.27 (s, 3H), 2.19 (s, 3H), 1.22 (s, 12H). 


\section{2-(3,5-Dimethyl-1H-pyrazol-1-yl)phenol (4b, Scheme 1a) $)^{17}$}<smiles>Cc1cc(C)n(-c2ccccc2O)n1</smiles>

Colorless solid; $31.0 \mathrm{mg}, 0.165 \mathrm{mmol}, 83 \%$ yield, purified by PTLC ( $n$-hexane/EtOAc $=4: 1) ;{ }^{1} \mathrm{H}$ NMR $\left(400 \mathrm{MHz}, \mathrm{CDCl}_{3}\right) \delta 9.80(\mathrm{~s}, 1 \mathrm{H}), 7.23-7.17(\mathrm{~m}, 2 \mathrm{H}), 7.09(\mathrm{dd}, J=8.5,1.4 \mathrm{~Hz}, 1 \mathrm{H}), 6.93-6.87$ $(\mathrm{m}, 1 \mathrm{H}), 6.03(\mathrm{~s}, 1 \mathrm{H}), 2.39(\mathrm{~s}, 3 \mathrm{H}), 2.30(\mathrm{~s}, 3 \mathrm{H}) ;{ }^{13} \mathrm{C} \mathrm{NMR}\left(\mathrm{CDCl}_{3}, 100 \mathrm{MHz}\right) \delta 150.8,149.3,140.6$, $128.2,125.7,122.6,119.2,118.3,107.5,13.4,13.0$.

2-[2-(4,4,5,5-Tetramethyl-1,3,2-dioxaborolan-2-yl)phenyl]pyridine (3c, Scheme 1a) ${ }^{16}$<smiles>Brc1ccccc1-c1ncccc1Br</smiles>

>99\% NMR yield, reaction time: $18 \mathrm{~h} ;{ }^{1} \mathrm{H}$ NMR $\left(400 \mathrm{MHz}, \mathrm{CDCl}_{3}\right) \delta 8.68(\mathrm{~d}, J=5.4 \mathrm{~Hz}, 1 \mathrm{H})$, $7.97(\mathrm{dd}, J=7.9,7.9 \mathrm{~Hz}, 1 \mathrm{H}), 7.81-7.75(\mathrm{~m}, 1 \mathrm{H}), 7.73(\mathrm{~d}, J=7.2 \mathrm{~Hz}, 1 \mathrm{H}), 7.66(\mathrm{~d}, J=7.6 \mathrm{~Hz}, 1 \mathrm{H})$, 7.43-7.25 (m, 3H), $1.43(\mathrm{~s}, 12 \mathrm{H})$.

\section{2-(Pyridin-2-yl)phenol (4c, Scheme 1a) $)^{18}$}<smiles>Oc1ccccc1-c1ccccn1</smiles>

Yellow oil; $22.2 \mathrm{mg}, 0.130 \mathrm{mmol}, 65 \%$ yield, purified by PTLC deactivated with triethylamine ( $n$ hexane/EtOAc $=2: 1) ;{ }^{1} \mathrm{H} \mathrm{NMR}\left(\mathrm{CDCl}_{3}, 400 \mathrm{MHz}\right) \delta 14.35(\mathrm{~s}, 1 \mathrm{H}), 8.49(\mathrm{~d}, J=4.4 \mathrm{~Hz}, 1 \mathrm{H}), 7.90$ $(\mathrm{d}, J=8.3 \mathrm{~Hz}, 1 \mathrm{H}), 7.85-7.76(\mathrm{~m}, 2 \mathrm{H}), 7.30(\mathrm{ddd}, J=8.4,7.2,1.6 \mathrm{~Hz}, 1 \mathrm{H}), 7.25-7.19(\mathrm{~m}, 1 \mathrm{H}), 7.03$ $(\mathrm{dd}, J=8.4,1.1 \mathrm{~Hz}, 1 \mathrm{H}), 6.94-6.87(\mathrm{~m}, 1 \mathrm{H}) ;{ }^{13} \mathrm{C} \mathrm{NMR}\left(\mathrm{CDCl}_{3}, 100 \mathrm{MHz}\right) \delta 160.0,157.9,145.8$, $137.8,131.5,126.1,121.5,119.1,118.83,118.79,118.6$.

3-Methyl-2-[2-(4,4,5,5-tetramethyl-1,3,2-dioxaborolan-2-yl)phenyl]pyridine (3d, Scheme 1a) ${ }^{19}$<smiles>Cc1cccnc1-c1ccccc1Br</smiles>

$>99 \%$ NMR yield, reaction time: $18 \mathrm{~h} ;{ }^{1} \mathrm{H}$ NMR $\left(400 \mathrm{MHz}, \mathrm{CDCl}_{3}\right) \delta 8.59(\mathrm{~d}, J=4.7 \mathrm{~Hz}, 1 \mathrm{H})$, 7.84-7.70 (m, 3H), 7.39 (ddd, $J=7.3,7.3,0.7 \mathrm{~Hz}, 1 \mathrm{H}), 7.32-7.24(\mathrm{~m}, 2 \mathrm{H}), 2.70(\mathrm{~s}, 3 \mathrm{H}), 1.42(\mathrm{~s}$, $12 \mathrm{H})$.

2-(3-Methylpyridin-2-yl)phenol (4d, Scheme 1a) ${ }^{18}$ 
<smiles>Cc1cccnc1-c1ccccc1O</smiles>

Yellow solid; $32.4 \mathrm{mg}, 0.175 \mathrm{mmol}, 87 \%$ yield, purified by PTLC deactivated with triethylamine $(n$-hexane/EtOAc $=2: 1) ;{ }^{1} \mathrm{H}$ NMR $\left(\mathrm{CDCl}_{3}, 400 \mathrm{MHz}\right) \delta 12.28(\mathrm{br}, 1 \mathrm{H}), 8.42(\mathrm{dd}, J=4.8,1.1 \mathrm{~Hz}$, $1 \mathrm{H}), 7.68(\mathrm{~d}, J=7.7 \mathrm{~Hz}, 1 \mathrm{H}), 7.54(\mathrm{dd}, J=7.9,1.6 \mathrm{~Hz}, 1 \mathrm{H}), 7.32-7.24(\mathrm{~m}, 1 \mathrm{H}), 7.19(\mathrm{dd}, J=7.7$, $4.8 \mathrm{~Hz}, 1 \mathrm{H}), 7.07(\mathrm{dd}, J=8.2,1.2 \mathrm{~Hz}, 1 \mathrm{H}), 6.94-6.88(\mathrm{~m}, 1 \mathrm{H}), 2.56(\mathrm{~s}, 3 \mathrm{H}) ;{ }^{13} \mathrm{C} \mathrm{NMR}\left(\mathrm{CDCl}_{3}, 100\right.$ $\mathrm{MHz}) \delta 157.8,156.4,144.4,141.2,131.7,130.5,129.7,121.7,121.6,118.4,118.0,21.8$.

2-Methyl-6-[2-(4,4,5,5-tetramethyl-1,3,2-dioxaborolan-2-yl)phenyl]pyridine (3e, Scheme 1a)<smiles>Cc1cccc(-c2ccccc2Br)n1</smiles>

43\% NMR yield, reaction time: $18 \mathrm{~h}$; ${ }^{1} \mathrm{H}$ NMR $\left(400 \mathrm{MHz}, \mathrm{CDCl}_{3}\right) \delta 8.00-7.92(\mathrm{~m}, 1 \mathrm{H}), 7.83-7.69$ (m, 2H), 7.65-7.30 (m, 2H), $7.23(\mathrm{dd}, J=7.5,7.5,1 \mathrm{H}), 7.10-7.02(\mathrm{~m}, 1 \mathrm{H}), 2.63(\mathrm{~s}, 3 \mathrm{H}), 1.27$ (s, $12 \mathrm{H})$.

\section{2-(6-Methylpyridin-2-yl)phenol (4e, Scheme 1a) ${ }^{20}$}<smiles>Cc1cccc(-c2ccccc2O)n1</smiles>

Yellow oil; $14.6 \mathrm{mg}, 0.0790 \mathrm{mmol}, 39 \%$ yield, purified by PTLC ( $n$-hexane/dichloromethane $=$ 1:1); ${ }^{1} \mathrm{H} \mathrm{NMR}\left(\mathrm{CDCl}_{3}, 400 \mathrm{MHz}\right) \delta 14.74(\mathrm{br}, 1 \mathrm{H}), 7.78(\mathrm{dd}, J=8.1,1.6 \mathrm{~Hz}, 1 \mathrm{H}), 7.74-7.67(\mathrm{~m}, 2 \mathrm{H})$, $7.29(\mathrm{ddd}, J=8.1,7.4,1.7 \mathrm{~Hz}, 1 \mathrm{H}), 7.10-7.04(\mathrm{~m}, 1 \mathrm{H}), 7.01(\mathrm{dd}, J=8.1,1.1 \mathrm{~Hz}, 1 \mathrm{H}), 6.92-6.86(\mathrm{~m}$, $1 \mathrm{H}), 2.59(\mathrm{~s}, 3 \mathrm{H}) ;{ }^{13} \mathrm{C} \mathrm{NMR}\left(\mathrm{CDCl}_{3}, 100 \mathrm{MHz}\right) \delta 160.2,157.3,155.1,138.0,131.3,126.2,121.1$, $118.9,118.7,118.6,116.0,23.8$.

\section{2-[2-(4,4,5,5-Tetramethyl-1,3,2-dioxaborolan-2-yl)phenoxy]pyridine (3f, Scheme 1a)}<smiles>Brc1ccccc1Oc1ccccn1</smiles>

45\% NMR yield, reaction time: $18 \mathrm{~h} ;{ }^{1} \mathrm{H}$ NMR $\left(400 \mathrm{MHz}, \mathrm{CDCl}_{3}\right) \delta 8.51(\mathrm{dd}, J=5.6,1.8,1 \mathrm{H})$, $7.82(\mathrm{ddd}, J=7.0,7.0,1.6,1 \mathrm{H}), 7.75(\mathrm{dd}, J=7.5,2.0,1 \mathrm{H}), 7.43-7.31(\mathrm{~m}, 2 \mathrm{H}), 7.25-7.07(\mathrm{~m}, 3 \mathrm{H})$, $1.21(\mathrm{~s}, 12 \mathrm{H})$.

2-(Pyridin-2-yloxy)phenol (4f, Scheme 1a) $)^{21}$<smiles>Oc1ccccc1Oc1ccccn1</smiles> 
Colorless solid; $10.1 \mathrm{mg}, 0.0538 \mathrm{mmol}, 27 \%$ yield, purified by PTLC (dichloromethane/EtOAc = 3:1); ${ }^{1} \mathrm{H}$ NMR $\left(\mathrm{CDCl}_{3}, 400 \mathrm{MHz}\right) \delta 8.19-8.13(\mathrm{~m}, 1 \mathrm{H}), 8.06-7.92(\mathrm{~m}, 1 \mathrm{H}), 7.78-7.69(\mathrm{~m}, 1 \mathrm{H}), 7.18$ $7.07(\mathrm{~m}, 3 \mathrm{H}), 7.07-6.98(\mathrm{~m}, 2 \mathrm{H}), 6.90(\mathrm{ddd}, J=7.1,6.1,1.9 \mathrm{~Hz}, 1 \mathrm{H}) ;{ }^{13} \mathrm{C} \mathrm{NMR}\left(\mathrm{CDCl}_{3}, 100 \mathrm{MHz}\right)$ $\delta 163.5,148.7,147.1,142.9,140.4,126.6,122.2,120.7,119.2,118.7,112.1$.

2-[2-(4,4,5,5-Tetramethyl-1,3,2-dioxaborolan-2-yl)benzyl]pyridine $(3 g, \text { Scheme } 1 \mathrm{a})^{16}$<smiles>Brc1ccccc1Cc1ccccn1</smiles>

37\% NMR yield, reaction time: $72 \mathrm{~h}$; ${ }^{1} \mathrm{H}$ NMR $\left(400 \mathrm{MHz}, \mathrm{CDCl}_{3}\right) \delta 9.11(\mathrm{~d}, J=4.8 \mathrm{~Hz}, 1 \mathrm{H}), 7.83$ $(\mathrm{ddd}, J=7.5,7.5,1.5 \mathrm{~Hz}, 1 \mathrm{H}), 7.70(\mathrm{~d}, J=6.6 \mathrm{~Hz}, 1 \mathrm{H}), 7.48(\mathrm{~d}, J=7.5 \mathrm{~Hz}, 1 \mathrm{H}), 7.39(\mathrm{dd}, J=6.6$, $6.6 \mathrm{~Hz}, 1 \mathrm{H}), 7.29-7.12(\mathrm{~m}, 3 \mathrm{H}), 4.34(\mathrm{~s}, 2 \mathrm{H}), 1.25(\mathrm{~s}, 12 \mathrm{H})$.

\section{2-(Pyridin-2-ylmethyl)phenol (4g, Scheme 1a) $)^{22}$}<smiles>Oc1ccccc1Cc1ccccn1</smiles>

Colorless solid; $6.0 \mathrm{mg}, 0.032 \mathrm{mmol}, 16 \%$ yield, purified by PTLC (dichloromethane $/$ EtOAc $=$ 3:1); ${ }^{1} \mathrm{H}$ NMR $\left(\mathrm{CDCl}_{3}, 400 \mathrm{MHz}\right) \delta 11.64(\mathrm{br}, 1 \mathrm{H}), 8.46(\mathrm{dd}, J=4.9,0.6 \mathrm{~Hz}, 1 \mathrm{H}), 7.69(\mathrm{ddd}, J=7.7$, $7.7,1.8 \mathrm{~Hz}, 1 \mathrm{H}), 7.31(\mathrm{~d}, J=7.8 \mathrm{~Hz}, 1 \mathrm{H}), 7.22-7.11(\mathrm{~m}, 3 \mathrm{H}), 7.02-6.95(\mathrm{~m}, 1 \mathrm{H}), 6.81(\mathrm{ddd}, J=7.4$, 7.4, 1.2 Hz, 1H), $4.09(\mathrm{~s}, 2 \mathrm{H}) ;{ }^{13} \mathrm{C} \mathrm{NMR}\left(\mathrm{CDCl}_{3}, 100 \mathrm{MHz}\right) \delta 161.0,156.6,147.8,138.2,130.2,128.6$, $126.2,122.8,121.9,119.9,118.5,41.7$.

1-[2-(4,4,5,5-Tetramethyl-1,3,2-dioxaborolan-2-yl)benzyl]-1H-pyrazole $(3 \mathrm{~h}, \text { Scheme } 1 \mathrm{a})^{23}$<smiles>Brc1ccccc1Cn1cccn1</smiles>

$46 \%$ NMR yield, reaction time: $18 \mathrm{~h} ;{ }^{1} \mathrm{H}$ NMR $\left(400 \mathrm{MHz}, \mathrm{CDCl}_{3}\right) \delta 7.86(\mathrm{dd}, J=7.1,1.6 \mathrm{~Hz}, 1 \mathrm{H})$, $7.58-7.52(\mathrm{~m}, 1 \mathrm{H}), 7.49-7.16(\mathrm{~m}, 3 \mathrm{H}), 7.04(\mathrm{dd}, J=7.6 \mathrm{~Hz}, 1 \mathrm{H}), 6.25(\mathrm{dd}, J=2.2,1.1 \mathrm{~Hz}, 1 \mathrm{H}), 5.66$ $(\mathrm{s}, 2 \mathrm{H}), 1.34(\mathrm{~s}, 12 \mathrm{H})$.

\section{2-[(1H-Pyrazol-1-yl)methyl]phenol (4h, Scheme 1a) ${ }^{24}$}<smiles>Oc1ccccc1Cn1cccn1</smiles>

Colorless solid; $11.4 \mathrm{mg}, 0.0655 \mathrm{mmol}, 33 \%$ yield, purified by PTLC deactivated with triethylamine ( $n$-hexane/EtOAc $=2: 1) ;{ }^{1} \mathrm{H} \mathrm{NMR}\left(\mathrm{CDCl}_{3}, 400 \mathrm{MHz}\right) \delta 10.29(\mathrm{~s}, 1 \mathrm{H}), 7.53(\mathrm{~d}, J=2.2$ $\mathrm{Hz}, 1 \mathrm{H}), 7.47(\mathrm{~d}, J=2.2 \mathrm{~Hz}, 1 \mathrm{H}), 7.28-7.21(\mathrm{~m}, 1 \mathrm{H}), 7.17(\mathrm{dd}, J=7.5,1.5 \mathrm{~Hz}, 1 \mathrm{H}), 7.00(\mathrm{dd}, J=$ 8.1, $1.1 \mathrm{~Hz}, 1 \mathrm{H}), 6.86(\mathrm{ddd}, J=8.1,7.5,1.1 \mathrm{~Hz}, 1 \mathrm{H}), 6.24(\mathrm{dd}, J=2.2,2.2 \mathrm{~Hz}, 1 \mathrm{H}), 5.22(\mathrm{~s}, 2 \mathrm{H}) ;{ }^{13} \mathrm{C}$ $\mathrm{NMR}\left(\mathrm{CDCl}_{3}, 100 \mathrm{MHz}\right) \delta 156.6,139.2,130.6,130.1,129.5,123.3,120.2,118.9,105.7,53.4$. 
<smiles>Brc1ccccc1-c1nccc2ccccc12</smiles>

$>99 \%$ NMR yield, reaction time: $18 \mathrm{~h} ;{ }^{1} \mathrm{H}$ NMR $\left(400 \mathrm{MHz}, \mathrm{CDCl}_{3}\right) \delta 8.76(\mathrm{~d}, J=5.0 \mathrm{~Hz}, 1 \mathrm{H})$, $8.33(\mathrm{~d}, J=8.5 \mathrm{~Hz}, 1 \mathrm{H}), 8.28(\mathrm{~d}, J=8.0 \mathrm{~Hz}, 1 \mathrm{H}), 8.00(\mathrm{ddd}, J=8.0,8.0,1.5 \mathrm{~Hz}, 1 \mathrm{H}), 7.90(\mathrm{~d}, J=$ $8.0 \mathrm{~Hz}, 1 \mathrm{H}), 7.89-7.87(\mathrm{~m}, 2 \mathrm{H}), 7.55(\mathrm{dd}, J=8.0 \mathrm{~Hz}, 1 \mathrm{H}), 7.47(\mathrm{dd}, J=8.0,8.0 \mathrm{~Hz}, 1 \mathrm{H}), 7.38(\mathrm{dd}, J$ $=8.0,5.0 \mathrm{~Hz}, 1 \mathrm{H}), 1.43(\mathrm{~s}, 12 \mathrm{H})$.

\section{2-(Isoquinolin-1-yl)phenol (4i, Scheme 1a) $)^{22}$}<smiles>Oc1ccccc1-c1nccc2ccccc12</smiles>

Yellow solid; $30.3 \mathrm{mg}, \quad 0.137 \mathrm{mmol}, 69 \%$ yield, purified by PTLC ( $n$ hexane/EtOAc/dichloromethane = 2:1:1); ${ }^{1} \mathrm{H} \mathrm{NMR}\left(\mathrm{CDCl}_{3}, 400 \mathrm{MHz}\right) \delta 12.03(\mathrm{br}, 1 \mathrm{H}), 8.51-8.41$ $(\mathrm{m}, 2 \mathrm{H}), 7.88(\mathrm{~d}, J=8.2 \mathrm{~Hz}, 1 \mathrm{H}), 7.77-7.69(\mathrm{~m}, 2 \mathrm{H}), 7.65-7.57(\mathrm{~m}, 2 \mathrm{H}), 7.38(\mathrm{ddd}, J=8.3,7.4,1.7$ $\mathrm{Hz}, 1 \mathrm{H}), 7.17(\mathrm{dd}, J=8.2,1.1 \mathrm{~Hz}, 1 \mathrm{H}), 7.00(\mathrm{ddd}, J=7.7,7.7,1.2 \mathrm{~Hz}, 1 \mathrm{H}) ;{ }^{13} \mathrm{C} \mathrm{NMR}\left(\mathrm{CDCl}_{3}, 100\right.$ MHz) $\delta 158.6,157.8,139.4,138.0,131.6,131.0,130.6,127.7,127.6,127.4,126.2,121.3,120.2$, $118.7,118.1$.

\section{0-(4,4,5,5-Tetramethyl-1,3,2-dioxaborolan-2-yl)benzo $[h]$ quinoline $(3 \mathbf{j}, \text { Scheme } 1 \mathrm{a})^{16}$}<smiles>Brc1cccc2ccc3cccnc3c12</smiles>

$>99 \%$ NMR yield, reaction time: $72 \mathrm{~h} ;{ }^{1} \mathrm{H}$ NMR $\left(400 \mathrm{MHz}, \mathrm{CDCl}_{3}\right) \delta 8.90(\mathrm{dd}, J=4.5,1.5 \mathrm{~Hz}$, $1 \mathrm{H}), 8.19(\mathrm{dd}, J=7.8,1.5 \mathrm{~Hz}, 1 \mathrm{H}), 7.88(\mathrm{dd}, J=7.5,1.5 \mathrm{~Hz}, 1 \mathrm{H}), 7.81(\mathrm{~d}, J=8.7 \mathrm{~Hz}, 1 \mathrm{H}), 7.77(\mathrm{dd}$, $J=6.9,1.5 \mathrm{~Hz}, 1 \mathrm{H}), 7.65-7.72(\mathrm{~m}, 2 \mathrm{H}), 7.52(\mathrm{dd}, J=7.8,4.5 \mathrm{~Hz}, 1 \mathrm{H}), 1.55(\mathrm{~s}, 12 \mathrm{H})$.

\section{Benzo $[h]$ quinolin-10-ol $(4 \mathbf{j} \text {, Scheme 1a })^{26}$}<smiles>Oc1cccc2ccc3cccnc3c12</smiles>

Yellow solid; $27.1 \mathrm{mg}, 0.139 \mathrm{mmol}, 69 \%$ yield, purified by PTLC deactivated with triethylamine (n-hexane/EtOAc $=4: 1) ;{ }^{1} \mathrm{H} \mathrm{NMR}\left(\mathrm{CDCl}_{3}, 400 \mathrm{MHz}\right) \delta 14.88(\mathrm{~s}, 1 \mathrm{H}), 8.82-8.74(\mathrm{~m}, 1 \mathrm{H}), 8.23-8.15$ $(\mathrm{m}, 1 \mathrm{H}), 7.80-7.72(\mathrm{~m}, 1 \mathrm{H}), 7.64-7.54(\mathrm{~m}, 2 \mathrm{H}), 7.54-7.47(\mathrm{~m}, 1 \mathrm{H}), 7.38(\mathrm{~d}, J=7.8 \mathrm{~Hz}, 1 \mathrm{H}), 7.27-$ $7.21(\mathrm{~m}, 1 \mathrm{H}) ;{ }^{13} \mathrm{C} \mathrm{NMR}\left(\mathrm{CDCl}_{3}, 100 \mathrm{MHz}\right) \delta 159.5,148.4,145.0,136.2,135.1,129.9,129.1,126.3$, $124.6,120.8,118.1,116.0,114.0$.

$N, N$-Dimethyl-1-[2-(4,4,5,5-tetramethyl-1,3,2-dioxaborolan-2-yl)phenyl]methanamine $\quad(3 \mathrm{k}$, Scheme 1a) $)^{27}$ 
<smiles>CN(C)Cc1ccccc1Br</smiles>

49\% NMR yield, reaction time: $18 \mathrm{~h} ;{ }^{1} \mathrm{H}$ NMR $\left(400 \mathrm{MHz}, \mathrm{CDCl}_{3}\right) \delta 7.53(\mathrm{~d}, J=7.1 \mathrm{~Hz}, 1 \mathrm{H}), 7.23-$ $7.13(\mathrm{~m}, 2 \mathrm{H}), 7.00(\mathrm{~d}, J=7.6 \mathrm{~Hz}, 1 \mathrm{H}), 3.86(\mathrm{~s}, 2 \mathrm{H}), 2.56(\mathrm{~s}, 6 \mathrm{H}), 1.31(\mathrm{~s}, 12 \mathrm{H})$.

\section{2-[(Dimethylamino)methyl]phenol (4k, Scheme 1a) $)^{28}$}<smiles>CN(C)Cc1ccccc1O</smiles>

Colorless oil; $5.3 \mathrm{mg}, 0.035 \mathrm{mmol}, 18 \%$ yield, purified by PTLC (toluene/EtOAc $=10: 1) ;{ }^{1} \mathrm{H}$ NMR $\left(\mathrm{CDCl}_{3}, 400 \mathrm{MHz}\right) \delta 7.19-7.13(\mathrm{~m}, 1 \mathrm{H}), 6.95(\mathrm{dd}, J=7.8,7.4 \mathrm{~Hz}, 1 \mathrm{H}), 6.82(\mathrm{dd}, J=7.8,1.0 \mathrm{~Hz}, 1 \mathrm{H})$, $6.77(\mathrm{ddd}, J=7.4,6.8,1.0 \mathrm{~Hz}, 1 \mathrm{H}), 3.64(\mathrm{~s}, 2 \mathrm{H}), 2.32(\mathrm{~s}, 6 \mathrm{H}) ;{ }^{13} \mathrm{C} \mathrm{NMR}\left(\mathrm{CDCl}_{3}, 100 \mathrm{MHz}\right) \delta 158.1$, $128.7,128.3,122.0,118.9,116.0,62.9,44.5$.

$N$-Benzyl- $N$-methyl-1-[2-(4,4,5,5-tetramethyl-1,3,2-dioxaborolan-2-yl)phenyl]methanamine (31, Scheme 1a)<smiles>CN(Cc1ccccc1)Cc1ccccc1Br</smiles>

$31 \%$ NMR yield, reaction time: $72 \mathrm{~h} ;{ }^{1} \mathrm{H}$ NMR $\left(400 \mathrm{MHz}, \mathrm{CDCl}_{3}\right) \delta 7.69-7.63(\mathrm{~m}, 1 \mathrm{H}), 7.41-7.18$ $(\mathrm{m}, 8 \mathrm{H}), 3.72(\mathrm{~s}, 2 \mathrm{H}), 3.59(\mathrm{~s}, 2 \mathrm{H}), 2.26(\mathrm{~s}, 3 \mathrm{H}), 1.35(\mathrm{~s}, 12 \mathrm{H})$.

\section{2-\{[Benzyl(methyl)amino]methyl $\}$ phenol (4l, Scheme 1a) ${ }^{29}$}<smiles>CN(Cc1ccccc1)Cc1ccccc1O</smiles>

Colorless oil; $9.7 \mathrm{mg}, 0.043 \mathrm{mmol}, 21 \%$ yield, purified by PTLC deactivated with triethylamine ( $n$-hexane/EtOAc $=4: 1) ;{ }^{1} \mathrm{H} \mathrm{NMR}\left(\mathrm{CDCl}_{3}, 400 \mathrm{MHz}\right) \delta 11.16(\mathrm{br}, 1 \mathrm{H}), 7.37-7.26(\mathrm{~m}, 5 \mathrm{H}), 7.17$ (ddd, $J=8.1,7.7,1.6 \mathrm{~Hz}, 1 \mathrm{H}), 6.99(\mathrm{~d}, J=7.4 \mathrm{~Hz}, 1 \mathrm{H}), 6.85(\mathrm{dd}, J=8.1,1.0 \mathrm{~Hz}, 1 \mathrm{H}), 6.78$ (ddd, $J=7.7$, $7.4,1.0 \mathrm{~Hz}, 1 \mathrm{H}), 3.75(\mathrm{~s}, 2 \mathrm{H}), 3.60(\mathrm{~s}, 2 \mathrm{H}), 2.24(\mathrm{~s}, 3 \mathrm{H}) ;{ }^{13} \mathrm{C} \mathrm{NMR}\left(\mathrm{CDCl}_{3}, 100 \mathrm{MHz}\right) \delta 157.9,136.9$, $129.4,128.8,128.6,128.6,127.7,121.9,119.1,116.1,61.5,60.9,41.3$.

1-[2-Methyl-6-(4,4,5,5-tetramethyl-1,3,2-dioxaborolan-2-yl)phenyl]-1H-pyrazole $(3 n$, Scheme 1a) $)^{30}$<smiles>Cc1cccc(Br)c1-n1cccn1</smiles>

$63 \%$ NMR yield, reaction time: $72 \mathrm{~h}$; ${ }^{1} \mathrm{H}$ NMR $\left(400 \mathrm{MHz}, \mathrm{CDCl}_{3}\right) \delta 7.66(\mathrm{dd}, J=1.9,0.6 \mathrm{~Hz}, 1 \mathrm{H})$, 7.59-7.56 (m, 2H), 7.35-7.29 (m, 2H), $6.38(\mathrm{dd}, J=2.1,1.9 \mathrm{~Hz}, 1 \mathrm{H}), 2.13(\mathrm{~s}, 3 \mathrm{H}), 1.19(\mathrm{~s}, 12 \mathrm{H})$. 
<smiles>Cc1cccc(O)c1-n1cccn1</smiles>

Yellow solid; $16.8 \mathrm{mg}, 0.0963 \mathrm{mmol}, 48 \%$ yield, purified by PTLC ( $n$-hexane/EtOAc $=4: 1) ;{ }^{1} \mathrm{H}$ NMR $\left(\mathrm{CDCl}_{3}, 400 \mathrm{MHz}\right) \delta 8.21(\mathrm{~s}, 1 \mathrm{H}), 7.79(\mathrm{~d}, J=1.9 \mathrm{~Hz}, 1 \mathrm{H}), 7.67(\mathrm{~d}, J=2.3 \mathrm{~Hz}, 1 \mathrm{H}), 7.15(\mathrm{dd}$, $J=8.0,7.8 \mathrm{~Hz}, 1 \mathrm{H}), 6.92(\mathrm{~d}, J=8.0 \mathrm{~Hz}, 1 \mathrm{H}), 6.83(\mathrm{~d}, J=7.8 \mathrm{~Hz}, 1 \mathrm{H}), 6.52(\mathrm{dd}, J=2.3,1.9 \mathrm{~Hz}, 1 \mathrm{H})$, $2.28(\mathrm{~s}, 3 \mathrm{H}) ;{ }^{13} \mathrm{C} \mathrm{NMR}\left(\mathrm{CDCl}_{3}, 100 \mathrm{MHz}\right) \delta 151.4,140.8,132.1,132.0,128.9,126.6,122.6,115.4$, $106.7,18.8$.

1-[5-Methyl-2-(4,4,5,5-tetramethyl-1,3,2-dioxaborolan-2-yl)phenyl]-1H-pyrazole

(3o, Scheme 1a)<smiles>Cc1ccc(Br)c(-n2cccn2)c1</smiles>

$71 \%$ NMR yield, reaction time: $18 \mathrm{~h}$; colorless amorphous; $13.6 \mathrm{mg}, 0.048 \mathrm{mmol}, 24 \%$ isolated yield, purified separately by PTLC deactivated with triethylamine ( $n$-hexane/EtOAc $=7: 3) ;{ }^{1} \mathrm{H}$ NMR $\left(400 \mathrm{MHz}, \mathrm{CDCl}_{3}\right) \delta 7.80(\mathrm{dd}, J=2.3 \mathrm{~Hz}, J=0.3 \mathrm{~Hz}, 1 \mathrm{H}), 7.64(\mathrm{~d}, J=1.6 \mathrm{~Hz}, 1 \mathrm{H}), 7.49(\mathrm{~d}, J=7.5$ $\mathrm{Hz}, 1 \mathrm{H}), 7.21(\mathrm{~s}, 1 \mathrm{H}), 7.10(\mathrm{dd}, J=7.5 \mathrm{~Hz}, J=0.7 \mathrm{~Hz}, 1 \mathrm{H}), 6.40(\mathrm{dd}, J=2.3,2.0 \mathrm{~Hz}, 1 \mathrm{H}), 2.38(\mathrm{~s}$, $3 \mathrm{H}), 1.33(\mathrm{~s}, 12 \mathrm{H}) ;{ }^{13} \mathrm{C} \mathrm{NMR}\left(\mathrm{CDCl}_{3}, 100 \mathrm{MHz}\right) \delta 143.5,140.6,139.4,134.3,127.4,127.3,120.0$, $107.3,83.6,21.6$. The carbon directly attached to the boron atom was not detected, likely due to quadrupolar relaxation; ${ }^{11} \mathrm{~B}$ NMR $\left(\mathrm{CDCl}_{3}, 160 \mathrm{MHz}\right) \delta 29.0 ; \mathrm{HRMS}(\mathrm{ESI}) \mathrm{m} / \mathrm{z}$ : $[\mathrm{M}+\mathrm{Na}]^{+} \mathrm{Calcd}$ for $\mathrm{C}_{16} \mathrm{H}_{21} \mathrm{BN}_{2} \mathrm{NaO}_{2}$ 307.1588; Found 307.1588.

\section{4-Methyl-2-(1H-pyrazol-1-yl)phenol (4o, Scheme 1a $)^{32}$}<smiles>Cc1ccc(O)c(-n2cccn2)c1</smiles>

Yellow oil; $17.2 \mathrm{mg}, 0.0987 \mathrm{mmol}, 49 \%$ yield, purified by PTLC ( $n$-hexane/dichloromethane $=$ 1:1); ${ }^{1} \mathrm{H} \mathrm{NMR}\left(\mathrm{CDCl}_{3}, 400 \mathrm{MHz}\right) \delta 11.10(\mathrm{~s}, 1 \mathrm{H}), 7.98(\mathrm{~d}, J=2.4 \mathrm{~Hz}, 1 \mathrm{H}), 7.71(\mathrm{~d}, J=2.0 \mathrm{~Hz}, 1 \mathrm{H})$, $7.17(\mathrm{~s}, 1 \mathrm{H}), 7.02-6.94(\mathrm{~m}, 2 \mathrm{H}), 6.48(\mathrm{dd}, J=2.4,2.0 \mathrm{~Hz}, 1 \mathrm{H}), 2.32(\mathrm{~s}, 3 \mathrm{H}) ;{ }^{13} \mathrm{C} \mathrm{NMR}\left(\mathrm{CDCl}_{3}, 100\right.$ MHz) $\delta 147.0,138.9,128.9,128.2,126.6,124.5,118.8,118.3,106.7,20.6$.

2-[4-Methyl-2-(4,4,5,5-tetramethyl-1,3,2-dioxaborolan-2-yl)phenyl]pyridine $\quad(3 p, \quad$ Scheme 1a) ${ }^{11}$<smiles>Cc1ccc(-c2ccccn2)c(Br)c1</smiles>

$>99 \%$ NMR yield, reaction time: $18 \mathrm{~h} ;{ }^{1} \mathrm{H}$ NMR $\left(400 \mathrm{MHz}, \mathrm{CDCl}_{3}\right) \delta 8.61(\mathrm{~d}, J=5.5 \mathrm{~Hz}, 1 \mathrm{H})$, $7.89(\mathrm{dd}, J=7.9,7.6 \mathrm{~Hz}, 1 \mathrm{H}), 7.71(\mathrm{~d}, J=7.9 \mathrm{~Hz}, 1 \mathrm{H}), 7.59-7.47(\mathrm{~m}, 2 \mathrm{H}), 7.28(\mathrm{dd}, J=6.5 \mathrm{~Hz}, 1 \mathrm{H})$, $7.08(\mathrm{~d}, J=7.6 \mathrm{~Hz}, 1 \mathrm{H}), 2.38(\mathrm{~s}, 3 \mathrm{H}), 1.43(\mathrm{~s}, 12 \mathrm{H})$. 
<smiles>Cc1ccc(-c2ccccn2)c(O)c1</smiles>

Yellow oil; $15.5 \mathrm{mg}, 0.138 \mathrm{mmol}, 69 \%$ yield, purified by PTLC deactivated with triethylamine ( $n$ hexane/EtOAc $=4: 1) ;{ }^{1} \mathrm{H} \mathrm{NMR}\left(\mathrm{CDCl}_{3}, 400 \mathrm{MHz}\right) \delta 14.31(\mathrm{~s}, 1 \mathrm{H}), 8.46(\mathrm{~d}, J=5.0 \mathrm{~Hz}, 1 \mathrm{H}), 7.84(\mathrm{~d}$, $J=8.3 \mathrm{~Hz}, 1 \mathrm{H}), 7.80-7.74(\mathrm{~m}, 1 \mathrm{H}), 7.66(\mathrm{~d}, J=8.1 \mathrm{~Hz}, 1 \mathrm{H}), 7.20-7.15(\mathrm{~m}, 1 \mathrm{H}), 6.84(\mathrm{~d}, J=1.2 \mathrm{~Hz}$, $1 \mathrm{H}), 6.71(\mathrm{dd}, J=8.1,1.2 \mathrm{~Hz}, 1 \mathrm{H}), 2.33(\mathrm{~s}, 3 \mathrm{H}) ;{ }^{13} \mathrm{C} \mathrm{NMR}\left(\mathrm{CDCl}_{3}, 100 \mathrm{MHz}\right) \delta 159.9,158.0,145.8$, $142.1,137.6,125.9,121.1,119.9,118.9,118.7,116.3,21.4$.

2-[2-Fluoro-6-(4,4,5,5-tetramethyl-1,3,2-dioxaborolan-2-yl)phenyl]pyridine $\quad(3 q, \quad$ Scheme 1a) $)^{33}$<smiles>Fc1cccc(Br)c1-c1ncccc1Br</smiles>

93\% NMR yield, $\left[\mathrm{Cp}^{\mathrm{A} 3} \mathrm{RhCl}_{2}\right]_{2}: 0.0100 \mathrm{mmol}, \mathrm{NaO}{ }^{t} \mathrm{Bu}: 0.040 \mathrm{mmol}, \mathrm{Ir}(\mathrm{dFppy})_{3}: 0.0040 \mathrm{mmol}$, reaction time: $72 \mathrm{~h} ;{ }^{1} \mathrm{H}$ NMR $\left(400 \mathrm{MHz}, \mathrm{CDCl}_{3}\right) \delta 8.68(\mathrm{~d}, J=5.5 \mathrm{~Hz}, 1 \mathrm{H}), 8.05(\mathrm{~d}, J=8.1 \mathrm{~Hz}, 1 \mathrm{H})$, $7.98(\mathrm{dd}, J=7.4,7.4 \mathrm{~Hz}, 1 \mathrm{H}), 7.49(\mathrm{~d}, J=7.1 \mathrm{~Hz}, 1 \mathrm{H}), 7.43-7.32(\mathrm{~m}, 2 \mathrm{H}), 6.95$ (dd, $J=11.0,8.1$ $\mathrm{Hz}, 1 \mathrm{H}), 1.41(\mathrm{~s}, 12 \mathrm{H})$.

\section{3-Fluoro-2-(pyridin-2-yl)phenol (4q, Scheme 1a) ${ }^{26}$}<smiles>Oc1cccc(F)c1-c1ccccn1</smiles>

Colorless solid; $22.2 \mathrm{mg}, 0.117 \mathrm{mmol}, 59 \%$ yield, purified by PTLC deactivated with triethylamine $(n$-hexane/EtOAc $=4: 1) ;{ }^{1} \mathrm{H}$ NMR $\left(\mathrm{CDCl}_{3}, 400 \mathrm{MHz}\right): \delta 14.84(\mathrm{~s}, 1 \mathrm{H}), 8.54(\mathrm{~d}, J=4.3 \mathrm{~Hz}, 1 \mathrm{H}), 8.16$ $(\mathrm{d}, J=8.4 \mathrm{~Hz}, 1 \mathrm{H}), 7.85(\mathrm{ddd}, J=8.4,8.3,0.8 \mathrm{~Hz}, 1 \mathrm{H}), 7.31-7.17(\mathrm{~m}, 2 \mathrm{H}), 6.83(\mathrm{~d}, J=8.3 \mathrm{~Hz}, 1 \mathrm{H})$, $6.64(\mathrm{ddd}, J=12.7,7.8,1.1 \mathrm{~Hz}, 1 \mathrm{H}) ;{ }^{13} \mathrm{C} \mathrm{NMR}\left(\mathrm{CDCl}_{3}, 100 \mathrm{MHz}\right) \delta 162.0(\mathrm{~d}, J=250.2 \mathrm{~Hz}), 161.4$ $(\mathrm{d}, J=5.4 \mathrm{~Hz}), 154.6(\mathrm{~d}, J=3.5 \mathrm{~Hz}), 145.4,138.0(\mathrm{~d}, J=2.0 \mathrm{~Hz}), 130.9(\mathrm{~d}, J=12.7 \mathrm{~Hz}), 124.6(\mathrm{~d}, J$ $=21.2 \mathrm{~Hz}), 122.0(\mathrm{~d}, J=1.1 \mathrm{~Hz}), 114.2(\mathrm{~d}, J=2.9 \mathrm{~Hz}), 108.8(\mathrm{~d}, J=11.3 \mathrm{~Hz}), 106.2(\mathrm{~d}, J=25.0$ $\mathrm{Hz}) ;{ }^{19} \mathrm{~F} \mathrm{NMR}\left(\mathrm{CDCl}_{3}, 376 \mathrm{MHz}\right) \delta-111.9$.

\section{1-[2-(4,4,5,5-Tetramethyl-1,3,2-dioxaborolan-2-yl)-5-(trifluoromethyl)phenyl]-1H-pyrazole} (3r, Scheme 1a)<smiles>FC(F)(F)c1ccc(Br)c(-n2cccn2)c1</smiles>

$80 \%$ NMR yield, reaction time: $18 \mathrm{~h}$; brown solid; $8.4 \mathrm{mg}, 0.025 \mathrm{mmol}, 12 \%$ isolated yield, purified separately by PTLC deactivated with triethylamine ( $n$-hexane/EtOAc $=7: 3) ; \mathrm{mp} .130 .2-132.0{ }^{\circ} \mathrm{C} ;{ }^{1} \mathrm{H}$ NMR $\left(400 \mathrm{MHz}, \mathrm{CDCl}_{3}\right) \delta 7.94(\mathrm{~d}, J=2.5 \mathrm{~Hz}, 1 \mathrm{H}), 7.71(\mathrm{~d}, J=1.7 \mathrm{~Hz}, 1 \mathrm{H}), 7.68(\mathrm{~d}, J=7.7 \mathrm{~Hz}$, $1 \mathrm{H}), 7.59(\mathrm{~s}, 1 \mathrm{H}), 7.51(\mathrm{~d}, J=7.7 \mathrm{~Hz}, 1 \mathrm{H}), 6.49(\mathrm{dd}, J=2.5,1.7 \mathrm{~Hz}, 1 \mathrm{H}), 1.38(\mathrm{~s}, 12 \mathrm{H}) ;{ }^{13} \mathrm{C} \mathrm{NMR}$ 
$\left(\mathrm{CDCl}_{3}, 100 \mathrm{MHz}\right) \delta 142.8,139.6,134.5,132.3(\mathrm{~d}, J=32.9 \mathrm{~Hz}), 126.3,123.8(\mathrm{q}, J=272.4 \mathrm{~Hz})$, $123.0(\mathrm{~d}, J=3.7 \mathrm{~Hz}), 113.6(\mathrm{~d}, J=3.8 \mathrm{~Hz}), 108.8,83.8,25.0$. The carbon directly attached to the boron atom was not detected, likely due to quadrupolar relaxation; ${ }^{19} \mathrm{~F}$ NMR $\left(\mathrm{CDCl}_{3}, 376 \mathrm{MHz}\right) \delta$ $-62.8 ;{ }^{11} \mathrm{~B}$ NMR $\left(\mathrm{CDCl}_{3}, 160 \mathrm{MHz}\right) \delta 26.7$; HRMS (ESI) m/z: $[\mathrm{M}+\mathrm{Na}]^{+} \mathrm{Calcd}$ for $\mathrm{C}_{16} \mathrm{H}_{18} \mathrm{BF}_{3} \mathrm{~N}_{2} \mathrm{NaO}_{2}$ 361.1306; Found 361.1311.

\section{2-(1H-Pyrazol-1-yl)-4-(trifluoromethyl)phenol (4r, Scheme 1a)}<smiles>Oc1ccc(C(F)(F)F)cc1-n1cccn1</smiles>

Colorless solid; $27.6 \mathrm{mg}, 0.121 \mathrm{mmol}, 60 \%$ yield, purified by PTLC ( $n$-hexane/dichloromethane $=$ 1:1); mp. 77.1-77.8 ${ }^{\circ} \mathrm{C} ;{ }^{1} \mathrm{H} \mathrm{NMR}\left(\mathrm{CDCl}_{3}, 400 \mathrm{MHz}\right) \delta 11.93(\mathrm{~s}, 1 \mathrm{H}), 8.06(\mathrm{~d}, J=2.5 \mathrm{~Hz}, 1 \mathrm{H}), 7.76$ $(\mathrm{d}, J=2.0 \mathrm{~Hz}, 1 \mathrm{H}), 7.62(\mathrm{~d}, J=1.6 \mathrm{~Hz}, 1 \mathrm{H}), 7.43(\mathrm{dd}, J=8.6,1.6 \mathrm{~Hz}, 1 \mathrm{H}), 7.17(\mathrm{dd}, J=8.6,0.4 \mathrm{~Hz}$, $1 \mathrm{H}), 6.56(\mathrm{dd}, J=2.5,2.0 \mathrm{~Hz}, 1 \mathrm{H}) ;{ }^{13} \mathrm{C} \mathrm{NMR}\left(\mathrm{CDCl}_{3}, 100 \mathrm{MHz}\right) \delta 152.2,152.2,139.4,126.9,124.6$ $(\mathrm{q}, J=4.2 \mathrm{~Hz}), 124.1(\mathrm{q}, J=271.2 \mathrm{~Hz}), 121.9(\mathrm{q}, J=33.3 \mathrm{~Hz}), 119.5,115.1(\mathrm{q}, J=3.9 \mathrm{~Hz}), 107.4$; ${ }^{19} \mathrm{~F}$ NMR $\left(\mathrm{CDCl}_{3}, 376 \mathrm{MHz}\right) \delta-61.5$; HRMS (ESI) m/z: [M-H] $]^{-}$Calcd for $\mathrm{C}_{10} \mathrm{H}_{6} \mathrm{~F}_{3} \mathrm{~N}_{2} \mathrm{O} 227.0438$; Found 227.0456.

1-[5-Chloro-2-(4,4,5,5-tetramethyl-1,3,2-dioxaborolan-2-yl)phenyl]-1H-pyrazole (3s, Scheme 1a)<smiles>Clc1ccc(Br)c(-n2cccn2)c1</smiles>

54\% NMR yield, reaction time: $72 \mathrm{~h}$; brown amorphous; $6.0 \mathrm{mg}, 0.0196 \mathrm{mmol}, 10 \%$ isolated yield; purified separately as a mixture of 3s and pinacol (3s: $85 \mathrm{wt} \%$ ) by PTLC deactivated with triethylamine ( $n$-hexane/EtOAc $=7: 3 ;{ }^{1} \mathrm{H} \mathrm{NMR}\left(\mathrm{CDCl}_{3}, 400 \mathrm{MHz}\right): \delta 7.83(\mathrm{~d}, J=2.3 \mathrm{~Hz}, 1 \mathrm{H}), 7.66$ $(\mathrm{d}, J=1.4 \mathrm{~Hz}, 1 \mathrm{H}), 7.50(\mathrm{~d}, J=7.9 \mathrm{~Hz}, 1 \mathrm{H}), 7.40(\mathrm{~d}, J=1.5 \mathrm{~Hz}, 1 \mathrm{H}), 7.31-7.22(\mathrm{~m}, 1 \mathrm{H}), 6.46(\mathrm{dd}, J$ $=2.3,1.4 \mathrm{~Hz}, 1 \mathrm{H}), 1.35(\mathrm{~s}, 12 \mathrm{H}) ;{ }^{13} \mathrm{C} \mathrm{NMR}\left(\mathrm{CDCl}_{3}, 100 \mathrm{MHz}\right) \delta 143.8,139.7,135.8,135.1,126.7$, $126.4,118.3,108.1,83.7,24.8$. The carbon directly attached to the boron atom was not detected, likely due to quadrupolar relaxation; ${ }^{11} \mathrm{~B} \mathrm{NMR}\left(\mathrm{CDCl}_{3}, 160 \mathrm{MHz}\right) \delta 28.5 ; \mathrm{HRMS}(\mathrm{ESI}) \mathrm{m} / \mathrm{z}:[\mathrm{M}+\mathrm{Na}]^{+}$ Calcd for $\mathrm{C}_{15} \mathrm{H}_{18} \mathrm{BClN}_{2} \mathrm{NaO}_{2}$ 327.1042; Found 327.1057.

2-[5-Methoxy-2-(4,4,5,5-tetramethyl-1,3,2-dioxaborolan-2-yl)phenyl]pyridine (3t, Scheme 1a) ${ }^{11}$<smiles>COc1ccc(Br)c(-c2ccccn2)c1</smiles>

74\% NMR yield, reaction time: $72 \mathrm{~h} ;{ }^{1} \mathrm{H}$ NMR $\left(400 \mathrm{MHz}, \mathrm{CDCl}_{3}\right) \delta 8.63(\mathrm{~d}, J=5.4 \mathrm{~Hz}, 1 \mathrm{H}), 7.95-$ $7.83(\mathrm{~m}, 1 \mathrm{H}), 7.71(\mathrm{~d}, J=7.9 \mathrm{~Hz}, 1 \mathrm{H}), 7.61(\mathrm{~d}, J=7.9 \mathrm{~Hz}, 1 \mathrm{H}), 7.34-7.28(\mathrm{~m}, 1 \mathrm{H}), 7.15(\mathrm{~s}, 1 \mathrm{H})$, $7.00-6.92(\mathrm{~m}, 1 \mathrm{H}), 3.84(\mathrm{~s}, 3 \mathrm{H}), 1.41(\mathrm{~s}, 12 \mathrm{H})$. 
<smiles>COc1ccc(O)c(-c2ccccn2)c1</smiles>

Yellow oil; $20.3 \mathrm{mg}, 0.101 \mathrm{mmol}, 50 \%$ yield, purified by a PTLC (toluene/EtOAc $=3: 1$ ); ${ }^{1} \mathrm{H}$ NMR $\left(\mathrm{CDCl}_{3}, 400 \mathrm{MHz}\right): \delta 13.80(\mathrm{~s}, 1 \mathrm{H}), 8.53-8.48(\mathrm{~m}, 1 \mathrm{H}), 7.89-7.78(\mathrm{~m}, 2 \mathrm{H}), 7.30(\mathrm{~d}, J=2.7 \mathrm{~Hz}, 1 \mathrm{H})$, 7.27-7.21 (m, 1H), 6.99-6.90 (m, 2H), $3.82(\mathrm{~s}, 3 \mathrm{H}) ;{ }^{13} \mathrm{C} \mathrm{NMR}\left(\mathrm{CDCl}_{3}, 100 \mathrm{MHz}\right) \delta 157.6,154.1$, $152.1,146.0,137.8,121.6,119.1,118.8,117.9,111.0,56.0$.

2-[2-(4,4,5,5-Tetramethyl-1,3,2-dioxaborolan-2-yl)-4-(trifluoromethyl)phenyl]pyridine (3u, Scheme 1a) $)^{35}$<smiles>FC(F)(F)c1ccc(-c2ccccn2)c(Br)c1</smiles>

$62 \%$ NMR yield, reaction time: $48 \mathrm{~h} ;{ }^{1} \mathrm{H}$ NMR $\left(400 \mathrm{MHz}, \mathrm{CDCl}_{3}\right) \delta 8.73(\mathrm{~d}, J=5.6 \mathrm{~Hz}, 1 \mathrm{H}), 8.06-$ $8.00(\mathrm{~m}, 1 \mathrm{H}), 7.94(\mathrm{~s}, 1 \mathrm{H}), 7.87(\mathrm{~d}, J=8.0 \mathrm{~Hz}, 1 \mathrm{H}), 7.75(\mathrm{~d}, J=8.0 \mathrm{~Hz}, 1 \mathrm{H}), 7.55(\mathrm{~d}, J=8.0 \mathrm{~Hz}, 1 \mathrm{H})$, 7.49-7.55 (m, 1H), $1.44(\mathrm{~s}, 12 \mathrm{H})$.

\section{2-(Pyridin-2-yl)-5-(trifluoromethyl)phenol (4u, Scheme 1a) ${ }^{26}$}<smiles>Oc1cc(C(F)(F)F)ccc1-c1ccccn1</smiles>

Yellow solid; $19.0 \mathrm{mg}, 0.0794 \mathrm{mmol}, 40 \%$ yield, purified by PTLC deactivated with triethylamine $(n$-hexane/EtOAc $=4: 1) ;{ }^{1} \mathrm{H}$ NMR $\left(\mathrm{CDCl}_{3}, 400 \mathrm{MHz}\right) \delta 14.59(\mathrm{br}, 1 \mathrm{H}), 8.55(\mathrm{~d}, J=4.7 \mathrm{~Hz}, 1 \mathrm{H})$, $8.00-7.83(\mathrm{~m}, 3 \mathrm{H}), 7.33(\mathrm{dd}, J=6.1,6.1 \mathrm{~Hz}, 1 \mathrm{H}), 7.27(\mathrm{~s}, 1 \mathrm{H}), 7.13(\mathrm{~d}, J=8.2 \mathrm{~Hz}, 1 \mathrm{H}) ;{ }^{13} \mathrm{C}$ NMR $\left(\mathrm{CDCl}_{3}, 100 \mathrm{MHz}\right) \delta 160.1,156.6,146.0,138.1,133.0(\mathrm{q}, J=32.6 \mathrm{~Hz}), 126.6,123.8(\mathrm{q}, J=272.4$ $\mathrm{Hz}), 122.5,121.5,119.6,115.8(\mathrm{q}, J=3.9 \mathrm{~Hz}), 115.0(\mathrm{q}, J=3.8 \mathrm{~Hz}) ;{ }^{19} \mathrm{~F} \mathrm{NMR}\left(\mathrm{CDCl}_{3}, 376 \mathrm{MHz}\right) \delta$ -63.2 .

1-\{3-(4,4,5,5-Tetramethyl-1,3,2-dioxaborolan-2-yl)-[1,1'-biphenyl]-4-yl\}-1H-pyrazole Scheme 1a)

$(3 \mathbf{v}$<smiles>Brc1cccc(-n2cccn2)c1</smiles>

$63 \%$ NMR yield, reaction time: $18 \mathrm{~h}$; brown solid; $33.0 \mathrm{mg}, 0.0953 \mathrm{mmol}, 48 \%$ isolated yield, purified separately as a mixture of $\mathbf{3 v}$ and pinacol (3v: $95 \mathrm{wt} \%)$ by PTLC deactivated with triethylamine ( $n$-hexane/EtOAc = 7:3); mp. 102.3-103.9 ${ }^{\circ} \mathrm{C} ;{ }^{1} \mathrm{H} \mathrm{NMR}\left(\mathrm{CDCl}_{3}, 400 \mathrm{MHz}\right) \delta 7.89(\mathrm{~d}$, $J=2.1 \mathrm{~Hz}, 1 \mathrm{H}), 7.79-7.76(\mathrm{~m}, 1 \mathrm{H}), 7.67(\mathrm{~d}, J=1.6 \mathrm{~Hz}, 1 \mathrm{H}), 7.64-7.59(\mathrm{~m}, 3 \mathrm{H}), 7.47-7.41(\mathrm{~m}, 3 \mathrm{H})$, $7.38-7.31(\mathrm{~m}, 1 \mathrm{H}), 6.46(\mathrm{dd}, J=2.1,1.6 \mathrm{~Hz}, 1 \mathrm{H}), 1.38(\mathrm{~s}, 12 \mathrm{H}) ;{ }^{13} \mathrm{C} \mathrm{NMR}\left(\mathrm{CDCl}_{3}, 100 \mathrm{MHz}\right) \delta 142.1$, 
$140.5,139.3,139.2,132.6,128.8,127.3,127.2,126.5,118.2,118.1,107.7,83.6,24.9$. The carbon directly attached to the boron atom was not detected, likely due to quadrupolar relaxation; ${ }^{11} \mathrm{~B}$ NMR $\left(\mathrm{CDCl}_{3}, 160 \mathrm{MHz}\right) \delta 28.4$; HRMS (ESI) m/z: $[\mathrm{M}+\mathrm{Na}]^{+}$Calcd for $\mathrm{C}_{21} \mathrm{H}_{23} \mathrm{BN}_{2} \mathrm{NaO}_{2}$ 369.1745; Found 369.1752 .

2-[4-Bromo-2-(4,4,5,5-tetramethyl-1,3,2-dioxaborolan-2-yl)phenyl]pyridine $\quad(3 \mathrm{w}$, Scheme 1a) $)^{35}$<smiles>Brc1ccc(-c2ccccn2)c(Br)c1</smiles>

64\% NMR yield, reaction time: $18 \mathrm{~h}$; ${ }^{1} \mathrm{H}$ NMR (400 MHz, $\left.\mathrm{CDCl}_{3}\right) \delta 8.65(\mathrm{~d}, J=5.2 \mathrm{~Hz}, 1 \mathrm{H}), 7.97$ $(\mathrm{dd}, J=7.6,7.6 \mathrm{~Hz}, 1 \mathrm{H}), 7.83-7.67(\mathrm{~m}, 2 \mathrm{H}), 7.51(\mathrm{~d}, J=8.0 \mathrm{~Hz}, 1 \mathrm{H}), 7.45-7.36(\mathrm{~m}, 2 \mathrm{H}), 1.42(\mathrm{~s}$, $12 \mathrm{H})$.

\section{5-Bromo-2-(pyridin-2-yl)phenol (4w, Scheme 1a) $)^{26}$}<smiles>Oc1cc(Br)ccc1-c1ccccn1</smiles>

Yellow oil; $20.9 \mathrm{mg}, 0.0836 \mathrm{mmol}, 42 \%$ yield, purified by PTLC deactivated with triethylamine ( $n$-hexane/EtOAc $=4: 1) ;{ }^{1} \mathrm{H}$ NMR $\left(\mathrm{CDCl}_{3}, 400 \mathrm{MHz}\right) \delta 14.61(\mathrm{br}, 1 \mathrm{H}), 8.52-8.46(\mathrm{~m}, 1 \mathrm{H}), 7.90$ $7.77(\mathrm{~m}, 2 \mathrm{H}), 7.65-7.59(\mathrm{~m}, 1 \mathrm{H}), 7.33-7.22(\mathrm{~m}, 1 \mathrm{H}), 7.21-7.17(\mathrm{~m}, 1 \mathrm{H}), 7.05-6.98(\mathrm{~m}, 1 \mathrm{H}) ;{ }^{13} \mathrm{C}$ NMR $\left(\mathrm{CDCl}_{3}, 100 \mathrm{MHz}\right) \delta 160.9,157.1,145.8,138.0,127.1,124.9,122.0,121.9,121.6,119.0,117.8$.

1-[4-Methoxy-2-(4,4,5,5-tetramethyl-1,3,2-dioxaborolan-2-yl)phenyl]-1H-pyrazole

$(3 x$, Scheme 1a)<smiles>COc1ccc(-n2cccn2)c(Br)c1</smiles>

60\% NMR yield, reaction time: $18 \mathrm{~h}$; brown amorphous; $10.0 \mathrm{mg}, 0.0331 \mathrm{mmol}, 16 \%$ isolated yield, purified separately as a mixture of $\mathbf{3 x}$ and pinacol (3x:91 wt\%) by PTLC deactivated with triethylamine $(n$-hexane/EtOAc $=3: 7) ;{ }^{1} \mathrm{H} \mathrm{NMR}\left(\mathrm{CDCl}_{3}, 400 \mathrm{MHz}\right) \delta 7.75(\mathrm{~d}, J=2.2 \mathrm{~Hz}, 1 \mathrm{H}), 7.63$ $(\mathrm{d}, J=1.9 \mathrm{~Hz}, 1 \mathrm{H}), 7.30(\mathrm{~d}, J=8.7 \mathrm{~Hz}, 1 \mathrm{H}), 7.11(\mathrm{~d}, J=2.9 \mathrm{~Hz}, 1 \mathrm{H}), 6.93(\mathrm{dd}, J=8.7,2.9 \mathrm{~Hz}, 1 \mathrm{H})$, $6.40(\mathrm{dd}, J=2.2,1.9 \mathrm{~Hz}, 1 \mathrm{H}), 3.85(\mathrm{~s}, 3 \mathrm{H}), 1.33(\mathrm{~s}, 12 \mathrm{H}) ;{ }^{13} \mathrm{C} \mathrm{NMR}\left(\mathrm{CDCl}_{3}, 100 \mathrm{MHz}\right) \delta 158.2$, $138.7,136.8,126.9,120.3,118.5,115.6,107.0,83.5,55.6,24.9$. The carbon directly attached to the boron atom was not detected, likely due to quadrupolar relaxation; ${ }^{11} \mathrm{~B} \mathrm{NMR}\left(\mathrm{CDCl}_{3}, 160 \mathrm{MHz}\right) \delta$ 28.0; HRMS (ESI) m/z: [M+Na] ${ }^{+}$Calcd for $\mathrm{C}_{16} \mathrm{H}_{21} \mathrm{BN}_{2} \mathrm{NaO}_{3} 323.1537$; Found 323.1543. Scheme 1a) 
<smiles>CC(C)(C)c1ccc(-n2cccn2)c(Br)c1</smiles>

73\% NMR yield, reaction time: $18 \mathrm{~h}$; brown amorphous; $7.8 \mathrm{mg}, 0.024 \mathrm{mmol}, 12 \%$ isolated yield, purified separately as a mixture of $\mathbf{3 y}$ and pinacol (3y: 46 wt $\%$ ) by PTLC deactivated with triethylamine ( $n$-hexane/EtOAc $=7: 3) ;{ }^{1} \mathrm{H}$ NMR $\left(400 \mathrm{MHz}, \mathrm{CDCl}_{3}\right) \delta 7.79(\mathrm{~d}, J=2.3 \mathrm{~Hz}, 1 \mathrm{H}), 7.62$ $(\mathrm{d}, J=1.6 \mathrm{~Hz}, 1 \mathrm{H}), 7.57(\mathrm{~d}, J=2.2 \mathrm{~Hz}, 1 \mathrm{H}), 7.42(\mathrm{dd}, J=8.4,2.2 \mathrm{~Hz}, 1 \mathrm{H}), 7.30(\mathrm{~d}, J=8.4 \mathrm{~Hz}, 1 \mathrm{H})$, $6.40(\mathrm{dd}, J=2.2,2.0 \mathrm{~Hz}, 1 \mathrm{H}), 1.35(\mathrm{~s}, 12 \mathrm{H}), 1.34(\mathrm{~s}, 9 \mathrm{H}) ;{ }^{13} \mathrm{C} \mathrm{NMR}\left(\mathrm{CDCl}_{3}, 100 \mathrm{MHz}\right) \delta 149.3$, $141.0,139.2,130.7,127.4,127.0,118.7,107.2,83.7,34.7,31.5,25.0$. The carbon directly attached to the boron atom was not detected, likely due to quadrupolar relaxation; ${ }^{11} \mathrm{~B} \mathrm{NMR}\left(\mathrm{CDCl}_{3}, 160 \mathrm{MHz}\right)$ $\delta$ 29.0; HRMS (ESI) m/z: [M+Na] ${ }^{+}$Calcd for $\mathrm{C}_{19} \mathrm{H}_{27} \mathrm{BN}_{2} \mathrm{NaO}_{2}$ 349.2058; Found 349.2059.

\section{5-(tert-Butyl)-2-(1H-pyrazol-1-yl)phenol (4y, Scheme 1a)}<smiles>CC(C)(C)c1ccc(-n2cccn2)c(O)c1</smiles>

Colorless oil; $22.9 \mathrm{mg}, 0.106 \mathrm{mmol}, 53 \%$ yield, purified by PTLC deactivated with triethylamine $(n$-hexane/EtOAc $=4: 1) ;{ }^{1} \mathrm{H} \mathrm{NMR}\left(\mathrm{CDCl}_{3}, 400 \mathrm{MHz}\right) \delta 11.25(\mathrm{~s}, 1 \mathrm{H}), 7.97(\mathrm{~d}, J=2.3 \mathrm{~Hz}, 1 \mathrm{H}), 7.70$ $(\mathrm{d}, J=2.0 \mathrm{~Hz}, 1 \mathrm{H}), 7.29(\mathrm{~d}, J=8.5 \mathrm{~Hz}, 1 \mathrm{H}), 7.14(\mathrm{~d}, J=2.1 \mathrm{~Hz}, 1 \mathrm{H}), 6.93(\mathrm{dd}, J=8.5,2.1 \mathrm{~Hz}, 1 \mathrm{H})$, $6.47(\mathrm{dd}, J=2.3,2.0 \mathrm{~Hz}, 1 \mathrm{H}), 1.32(\mathrm{~s}, 9 \mathrm{H}) ;{ }^{13} \mathrm{C} \mathrm{NMR}\left(\mathrm{CDCl}_{3}, 100 \mathrm{MHz}\right) \delta 151.3,148.8,138.7,126.4$, 122.5, 117.4, 116.6, 116.1, 106.5, 34.6, 31.2; HRMS (ESI) m/z: [M-H] $]^{-}$Calcd for $\mathrm{C}_{13} \mathrm{H}_{15} \mathrm{~N}_{2} \mathrm{O}$ 215.1190; Found 215.1199.

\section{1-[2-(4,4,5,5-Tetramethyl-1,3,2-dioxaborolan-2-yl)naphthalen-1-yl]-1H-pyrazole} Scheme 1a)<smiles>Brc1ccc2ccccc2c1-n1cccn1</smiles>

$>99 \%$ NMR yield, reaction time: $18 \mathrm{~h}$; brown amorphous; $30.4 \mathrm{mg}, 0.095 \mathrm{mmol}, 47 \%$ isolated yield, purified separately as a mixture of $\mathbf{3 z}$ and pinacol (3z: $54 \mathrm{wt} \%$ ) by PTLC deactivated with triethylamine ( $n$-hexane/EtOAc $=7: 3) ;{ }^{1} \mathrm{H} \mathrm{NMR}\left(400 \mathrm{MHz}, \mathrm{CDCl}_{3}\right) \delta 7.91(\mathrm{~d}, J=8.2,1 \mathrm{H}), 7.89(\mathrm{~d}$, $J=8.3,1 \mathrm{H}), 7.79(\mathrm{~d}, J=8.3,1 \mathrm{H}), 7.77(\mathrm{~d}, J=1.5,1 \mathrm{H}), 7.72(\mathrm{~d}, J=2.3 \mathrm{~Hz}, 1 \mathrm{H}), 7.52(\mathrm{ddd}, J=8.1$, $6.7,1.4 \mathrm{~Hz}, 1 \mathrm{H}), 7.45(\mathrm{ddd}, J=8.5,6.7,1.2 \mathrm{~Hz}, 1 \mathrm{H}), 7.39(\mathrm{~d}, J=8.5,1 \mathrm{H}), 6.48(\mathrm{dd}, J=2.1,2.1 \mathrm{~Hz}$, $1 \mathrm{H}), 1.22(\mathrm{~s}, 12 \mathrm{H}) ;{ }^{13} \mathrm{C} \mathrm{NMR}\left(\mathrm{CDCl}_{3}, 100 \mathrm{MHz}\right) \delta 142.7,139.9,135.4,132.8,130.4,130.0,128.4$, $127.9,127.3,127.3,123.6,105.7,84.0,25.0$. The carbon directly attached to the boron atom was not detected, likely due to quadrupolar relaxation; ${ }^{11} \mathrm{~B} \mathrm{NMR}\left(\mathrm{CDCl}_{3}, 160 \mathrm{MHz}\right) \delta 30.9$; HRMS (ESI) $\mathrm{m} / \mathrm{z}:[\mathrm{M}+\mathrm{Na}]^{+}$Calcd for $\mathrm{C}_{19} \mathrm{H}_{21} \mathrm{BN}_{2} \mathrm{NaO}_{2} 343.1588$; Found 343.1588 .

\section{1-(1H-pyrazol-1-yl)naphthalen-2-ol $(4 z, \text { Scheme } 1 a)^{36}$}


<smiles>Oc1ccc2ccccc2c1-n1cccn1</smiles>

Colorless solid; $30.7 \mathrm{mg}, 0.146 \mathrm{mmol}, 73 \%$ yield, purified by PTLC ( $n$-hexane/EtOAc $=4: 1) ;{ }^{1} \mathrm{H}$ $\operatorname{NMR}\left(\mathrm{CDCl}_{3}, 400 \mathrm{MHz}\right) \delta 8.66(\mathrm{~s}, 1 \mathrm{H}), 7.92(\mathrm{~d}, J=2.3 \mathrm{~Hz}, 1 \mathrm{H}), 7.90(\mathrm{~d}, J=2.0 \mathrm{~Hz}, 1 \mathrm{H}), 7.86-7.71$ (m, 3H), 7.51-7.44 (m, 1H), 7.41-7.35 (m, 1H), $7.30(\mathrm{~d}, J=8.9 \mathrm{~Hz}, 1 \mathrm{H}), 6.63(\mathrm{dd}, J=2.3,2.0 \mathrm{~Hz}$, $1 \mathrm{H}) ;{ }^{13} \mathrm{C} \mathrm{NMR}\left(\mathrm{CDCl}_{3}, 100 \mathrm{MHz}\right) \delta 148.9,141.2,132.8,129.8,129.0,128.5,128.4,127.5,123.9$, $120.8,119.5,119.0,107.0$.

\section{1-[2-(5,5-Dimethyl-1,3,2-dioxaborinan-2-yl)phenyl]-1H-pyrazole (6a, Scheme 1a)}<smiles>CC1(C)COB(c2ccccc2-n2cccn2)OC1</smiles>

29\% NMR yield, reaction time: $72 \mathrm{~h}$; yellow amorphous; $4.9 \mathrm{mg}, 0.020 \mathrm{mmol}, 10 \%$ isolated yield, purified separately as a mixture of 6a and 2,2-dimethylpropane-1,3-diol (6a: 14 wt\%) by PTLC deactivated with triethylamine ( $n$-hexane/EtOAc $=3: 7) ;{ }^{1} \mathrm{H} \mathrm{NMR}\left(\mathrm{CDCl}_{3}, 400 \mathrm{MHz}\right) \delta 7.87(\mathrm{~d}, J=$ $2.3 \mathrm{~Hz}, 1 \mathrm{H}), 7.68(\mathrm{~d}, J=1.4 \mathrm{~Hz}, 1 \mathrm{H}), 7.60(\mathrm{~d}, J=7.3 \mathrm{~Hz}, 1 \mathrm{H}), 7.42-7.34(\mathrm{~m}, 2 \mathrm{H}), 7.32-7.25(\mathrm{~m}, 1 \mathrm{H})$, $6.46(\mathrm{dd}, J=2.3,1.4 \mathrm{~Hz}, 1 \mathrm{H}), 3.68(\mathrm{~s}, 4 \mathrm{H}), 1.08(\mathrm{~s}, 6 \mathrm{H}) ;{ }^{13} \mathrm{C} \mathrm{NMR}\left(\mathrm{CDCl}_{3}, 100 \mathrm{MHz}\right) \delta 141.1,138.9$, $133.0,129.5,127.0,126.6,119.4,107.6,72.4,22.2,14.7$. The carbon directly attached to the boron atom was not detected, likely due to quadrupolar relaxation; ${ }^{11} \mathrm{~B} \mathrm{NMR}\left(\mathrm{CDCl}_{3}, 160 \mathrm{MHz}\right) \delta 17.9$; HRMS (ESI) m/z: [M+Na] ${ }^{+}$Calcd for $\mathrm{C}_{14} \mathrm{H}_{17} \mathrm{BN}_{2} \mathrm{NaO}_{2}$ 279.1275; Found 279.1272.

\section{Methyl 4-(1H-pyrazol-1-yl)-3-(4,4,5,5-tetramethyl-1,3,2-dioxaborolan-2-yl)benzoate} Scheme 1b)<smiles>COC(=O)c1ccc(-n2cccn2)c(Br)c1</smiles>

72\% NMR yield, reaction time: $18 \mathrm{~h}$; yellow amorphous; $15.9 \mathrm{mg}, 0.0485 \mathrm{mmol}, 24 \%$ isolated yield, purified separately as a mixture of $\mathbf{8}$ and pinacol (8: 62 wt\%) by PTLC deactivated with triethylamine $(n$-hexane/EtOAc $=3: 7) ;{ }^{1} \mathrm{H} \mathrm{NMR}\left(\mathrm{CDCl}_{3}, 400 \mathrm{MHz}\right) \delta 8.21(\mathrm{~d}, J=1.8 \mathrm{~Hz}, 1 \mathrm{H}), 8.09$ $(\mathrm{dd}, J=8.5,1.9 \mathrm{~Hz}, 1 \mathrm{H}), 7.94(\mathrm{~d}, J=2.3 \mathrm{~Hz}, 1 \mathrm{H}), 7.70(\mathrm{~d}, J=1.9 \mathrm{~Hz}, 1 \mathrm{H}), 7.43(\mathrm{~d}, J=8.5 \mathrm{~Hz}, 1 \mathrm{H})$, $6.50(\mathrm{dd}, J=2.3,1.8 \mathrm{~Hz}, 1 \mathrm{H}), 3.93(\mathrm{~s}, 3 \mathrm{H}), 1.40(\mathrm{~s}, 12 \mathrm{H}) ;{ }^{13} \mathrm{C} \mathrm{NMR}\left(\mathrm{CDCl}_{3}, 100 \mathrm{MHz}\right) \delta 166.5$, $145.4,139.7,135.3,131.6,127.7,126.1,116.1,108.7,83.7,52.1,24.9$. The carbon directly attached to the boron atom was not detected, likely due to quadrupolar relaxation; ${ }^{11} \mathrm{~B} \mathrm{NMR}\left(\mathrm{CDCl}_{3}, 160 \mathrm{MHz}\right)$ $\delta$ 27.5; HRMS (ESI) m/z: [M+Na] ${ }^{+}$Calcd for $\mathrm{C}_{17} \mathrm{H}_{21} \mathrm{BN}_{2} \mathrm{NaO}_{4}$ 351.1487; Found 351.1495.

Procedure for $[\operatorname{Ir}(\mathrm{OMe})(\operatorname{cod})]_{2} /$ Silica-SMAP-catalyzed ortho-borylation of 7 (Scheme 1b): To a Schlenk flask were added $[\mathrm{Ir}(\mathrm{OMe})(\mathrm{cod})]_{2}(0.2 \mathrm{mg}, 0.00025 \mathrm{mmol})$, Silica-SMAP $(0.08 \mathrm{mmol} / \mathrm{g}$, $6.3 \mathrm{mg}, 0.00050 \mathrm{mmol}), 2(25.4 \mathrm{mg}, 0.100 \mathrm{mmol}), 7(40.4 \mathrm{mg}, 0.200 \mathrm{mmol})$, and $n$-hexane $(0.3 \mathrm{~mL})$ or THF $(0.3 \mathrm{~mL})$ in this order. After the mixture was stirred at room temperature or $80{ }^{\circ} \mathrm{C}$ under $\mathrm{Ar}$ for $18 \mathrm{~h}$, the resulting mixture was diluted with EtOAc and filtered through a glass pipet equipped with a cotton filter. The solvent was removed under reduced pressure. To the residue were added 1,3,5-trimethylbenzene (as an internal standard) and $\mathrm{CDCl}_{3}$, then the reaction was analyzed by ${ }^{1} \mathrm{H}$ NMR and GC/MS. 
Figure S1. Results of GC/MS measurement of Silica-SMAP-catalyzed ortho-borylation of 7
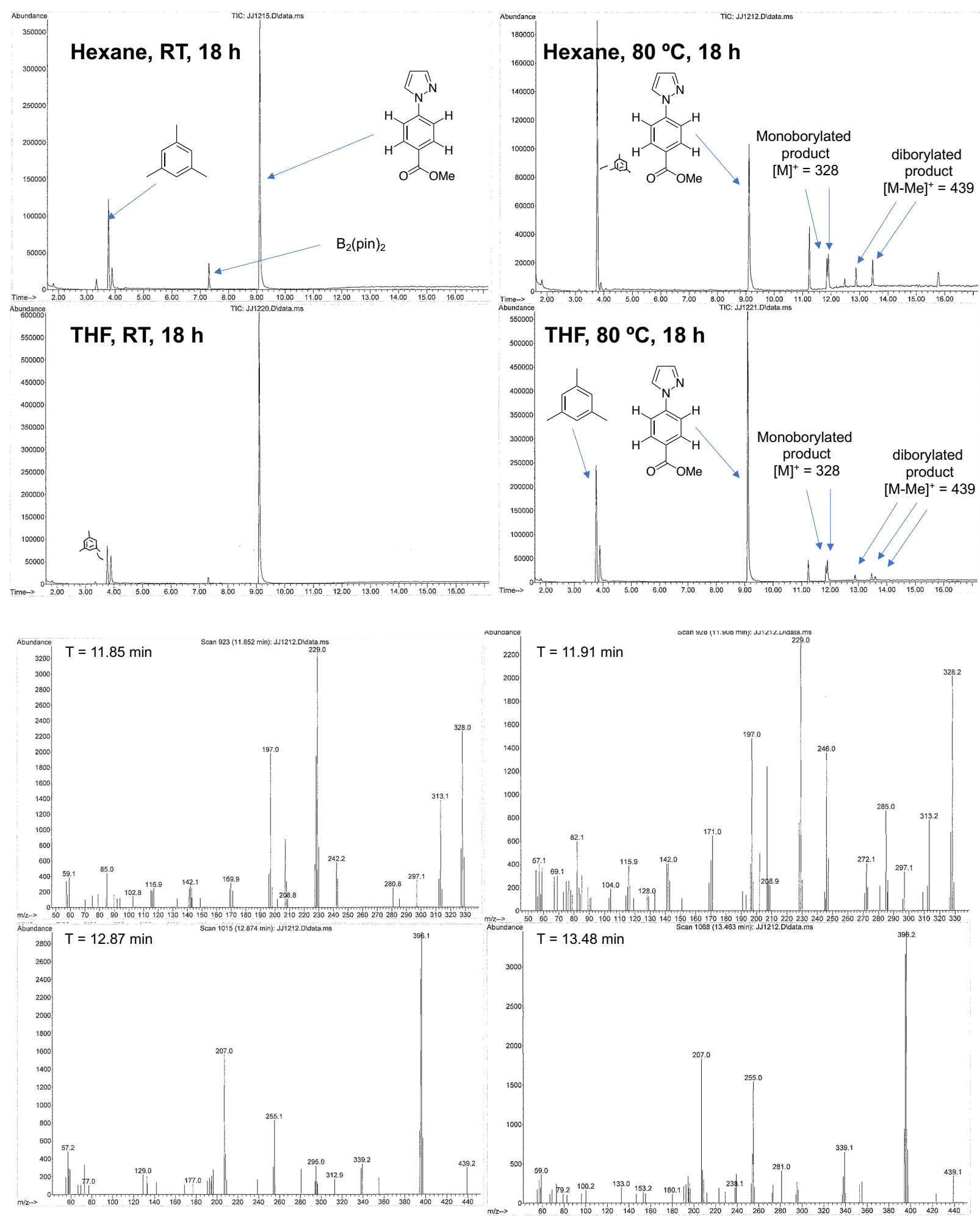


\section{Direct Various Elementalization Processes in One-Pot}

Procedure for borylation (Scheme 2): The borylation reactions were carried out according to the general procedure for Scheme 1 using $\left[\mathrm{Cp}^{\mathrm{A} 3} \mathrm{RhCl}_{2}\right]_{2}(6.0 \mathrm{mg}, 0.0050 \mathrm{mmol}), \mathrm{NaO}^{t} \mathrm{Bu}(1.9 \mathrm{mg}, 0.020$ mmol), $\operatorname{Ir}(\mathrm{dFppy})_{3}(1.5 \mathrm{mg}, 0.0020 \mathrm{mmol}), 2$ (101.6 mg, $\left.0.400 \mathrm{mmol}\right), \mathbf{1 b}(26.4 \mu \mathrm{L}, 0.200 \mathrm{mmol})$, and THF (2.0 mL) at room temperature under Ar with blue LEDs irradiation for $18 \mathrm{~h}$.

Procedure for one-pot chlorination (Scheme 2 condition b): To a Schlenk flask were added the crude residue, $\mathrm{CuCl}_{2}(80.7 \mathrm{mg}, 0.600 \mathrm{mmol}), \mathrm{MeCN}(2.4 \mathrm{~mL})$, and $\mathrm{H}_{2} \mathrm{O}(2.4 \mathrm{~mL})$. The mixture was stirred at $90{ }^{\circ} \mathrm{C}$ under air for $20 \mathrm{~h}$. The reaction was quenched with water, and the aqueous layer was extracted with EtOAc. The combined organic layer was washed with brine, dried over $\mathrm{Na}_{2} \mathrm{SO}_{4}$, and concentrated. The residue was purified by PTLC (toluene/EtOAc $=3: 1)$ to give $\mathbf{9 b}(18.1 \mathrm{mg}, 0.0876$ mmol, $44 \%$ yield) as a yellow oil.

\section{1-(2-Chlorophenyl)-3,5-dimethyl-1H-pyrazole (9b, Scheme 2$)^{37}$}

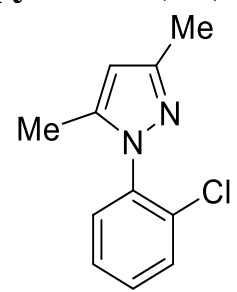

${ }^{1} \mathrm{H} \mathrm{NMR}\left(\mathrm{CDCl}_{3}, 400 \mathrm{MHz}\right) \delta$ 7.55-7.47 (m, 1H), 7.48-7.31 (m, 3H), $5.99(\mathrm{~s}, 1 \mathrm{H}), 2.30(\mathrm{~s}, 3 \mathrm{H})$, $2.11(\mathrm{~s}, 3 \mathrm{H}) ;{ }^{13} \mathrm{C} \mathrm{NMR}\left(\mathrm{CDCl}_{3}, 100 \mathrm{MHz}\right) \delta 149.4,141.2,137.6,132.6,130.2,130.1,130.0,127.5$, $105.5,13.6,11.3$.

Procedure for one-pot deuterium labeling reaction (Scheme 2, condition c): To a Schlenk flask were added the crude residue, $\mathrm{AgNO}_{3}(2.0 \mathrm{mg}, 0.012 \mathrm{mmol})$, triethylamine $(27.7 \mu \mathrm{L}, 0.2 \mathrm{mmol})$, $\mathrm{MeOD}(0.5 \mathrm{~mL})$, and $\mathrm{D}_{2} \mathrm{O}(0.5 \mathrm{~mL})$. The mixture was stirred at $80^{\circ} \mathrm{C}$ under air for $5 \mathrm{~h}$. The resulting solution was poured into brine, and the aqueous layer was extracted with EtOAc. The combined organic layer was washed with brine, dried over $\mathrm{Na}_{2} \mathrm{SO}_{4}$, and concentrated. The residue was purified by PTLC ( $n$-hexane/EtOAc $=4: 1)$ to give $10 b(27.5 \mathrm{mg}, 0.159 \mathrm{mmol}, 79 \%$ yield $)$ as a colorless oil.

\section{3,5-Dimethyl-1-(phenyl-2-d)-1H-pyrazole (10b, Scheme 2)}

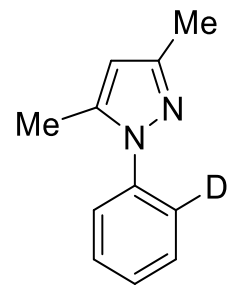

${ }^{1} \mathrm{H}$ NMR $\left(\mathrm{CDCl}_{3}, 400 \mathrm{MHz}\right) \delta$ 7.47-7.40 (m, 3H), 7.37-7.29 (m, 1H), $5.99(\mathrm{~s}, 1 \mathrm{H}), 2.30(\mathrm{~s}, 6 \mathrm{H})$; ${ }^{13} \mathrm{C} \mathrm{NMR}\left(\mathrm{CDCl}_{3}, 100 \mathrm{MHz}\right) \delta 149.0,140.0(\mathrm{~d}, J=6.2 \mathrm{~Hz}), 139.4,129.0(\mathrm{~d}, J=11.3 \mathrm{~Hz}), 127.2(\mathrm{~d}$, $J=1.4 \mathrm{~Hz}), 124.8(\mathrm{~d}, J=3.1 \mathrm{~Hz}), 106.9,13.5,12.4$; HRMS (ESI) m/z: $[\mathrm{M}+\mathrm{H}]{ }^{+}$Calcd for $\mathrm{C}_{11} \mathrm{H}_{12} \mathrm{DN}_{2}$ 174.1136; Found 174.1136.

Procedure for one-pot alkynylation (Scheme 2, condition d): To a Schlenk flask were added the crude residue, $\mathrm{Pd}_{2}(\mathrm{dba})_{3}(4.6 \mathrm{mg}, 0.0050 \mathrm{mmol})$, Xantphos $(11.6 \mathrm{mg}, 0.0200 \mathrm{mmol}), \mathrm{K}_{3} \mathrm{PO}_{4}(66.3 \mathrm{mg}$, $0.480 \mathrm{mmol})$, (bromoethynyl)triisopropylsilane $(191.8 \mu \mathrm{L}, 0.800 \mathrm{mmol}), 1,4$-dioxane $(0.9 \mathrm{~mL})$, and $\mathrm{H}_{2} \mathrm{O}(0.1 \mathrm{~mL})$. The mixture was stirred at $100{ }^{\circ} \mathrm{C}$ under $\mathrm{Ar}$ for $24 \mathrm{~h}$. The reaction was quenched with water, and the aqueous layer was extracted with EtOAc. The combined organic layer was washed with brine, dried over $\mathrm{Na}_{2} \mathrm{SO}_{4}$, and concentrated. The residue was purified by PTLC (toluene/EtOAc $=4: 1)$ to give $11 \mathrm{~b}(37.9 \mathrm{mg}, 0.107 \mathrm{mmol}, 54 \%$ yield $)$ as a colorless oil. 


\section{3,5-Dimethyl-1-\{2-[(triisopropylsilyl)ethynyl]phenyl\}-1H-pyrazole (11b, Scheme 2)}<smiles></smiles>

${ }^{1} \mathrm{H}$ NMR $\left(\mathrm{CDCl}_{3}, 400 \mathrm{MHz}\right) \delta 7.60-7.54(\mathrm{~m}, 1 \mathrm{H}), 7.43-7.32(\mathrm{~m}, 3 \mathrm{H}), 5.91(\mathrm{~s}, 1 \mathrm{H}), 2.25(\mathrm{~s}, 3 \mathrm{H})$, $2.15(\mathrm{~s}, 3 \mathrm{H}), 1.03-0.97(\mathrm{~m}, 21 \mathrm{H}) ;{ }^{13} \mathrm{C} \mathrm{NMR}\left(\mathrm{CDCl}_{3}, 100 \mathrm{MHz}\right) \delta 148.7,141.6,140.8,133.3,129.0$, 128.4, 128.2 , 122.7 , 105.5, 102.4, 95.6, 18.5, 13.5, 11.5, 11.2; HRMS (ESI) m/z: [M+Na]+ Calcd for $\mathrm{C}_{22} \mathrm{H}_{32} \mathrm{~N}_{2} \mathrm{NaSi}$ 375.2227; Found 375.2226.

Procedure for one-pot Suzuki-Miyaura coupling (Scheme 2, condition e): To a Schlenk flask were added the crude residue, $\mathrm{Pd}(\mathrm{OAc})_{2}(2.2 \mathrm{mg}, 0.010 \mathrm{mmol})$, SPhos (4.1 mg, $\left.0.010 \mathrm{mmol}\right), p$ bromoanisole $(37.7 \mu \mathrm{L}, 0.300 \mathrm{mmol}), 1$,4-dioxane $(1.8 \mathrm{~mL})$, and $2 \mathrm{~N} \mathrm{NaOH}(0.2 \mathrm{~mL})$. The mixture was stirred at $80^{\circ} \mathrm{C}$ under Ar for $19 \mathrm{~h}$. The reaction was quenched with water, and the aqueous layer was extracted with EtOAc. The combined organic layer was washed with brine, dried over $\mathrm{Na}_{2} \mathrm{SO}_{4}$, and concentrated. The residue was purified by PTLC $(n$-hexane/EtOAc $=4: 1)$ to give $\mathbf{1 2 b}(21.7 \mathrm{mg}$, $0.0780 \mathrm{mmol}, 39 \%$ yield) as a yellow oil.

\section{1-\{4'-Methoxy-[1,1'-biphenyl]-2-yl $\}-3,5-d i m e t h y l-1 H$-pyrazole $(12 b, \text { Scheme } 2)^{38}$}<smiles>COc1ccc(-c2ccccc2-n2nc(C)cc2C)cc1</smiles>

${ }^{1} \mathrm{H}$ NMR $\left(\mathrm{CDCl}_{3}, 400 \mathrm{MHz}\right) \delta 7.52-7.36(\mathrm{~m}, 4 \mathrm{H}), 7.05-6.97(\mathrm{~m}, 2 \mathrm{H}), 6.83-6.75(\mathrm{~m}, 2 \mathrm{H}), 5.76(\mathrm{~s}$, $1 \mathrm{H}), 3.79(\mathrm{~s}, 3 \mathrm{H}), 2.30(\mathrm{~s}, 3 \mathrm{H}), 1.62(\mathrm{~s}, 3 \mathrm{H}) ;{ }^{13} \mathrm{C} \mathrm{NMR}\left(\mathrm{CDCl}_{3}, 100 \mathrm{MHz}\right) \delta 158.9,148.6,140.6$, $138.8,137.4,130.9,130.2,129.6,129.0,128.9,127.7,113.8,105.4,55.2,13.6,11.1$.

Procedure for one-pot alkylation (Scheme 2, condition f): To a Schlenk flask were added the crude residue, $\mathrm{Pd}_{2}(\mathrm{dba})_{3}(4.6 \mathrm{mg}, 0.0050 \mathrm{mmol})$, DavePhos $(7.9 \mathrm{mg}, 0.020 \mathrm{mmol})$, benzyl bromide (47.5 $\mu \mathrm{L}, 0.400 \mathrm{mmol}), \mathrm{K}_{2} \mathrm{CO}_{3}(103.7 \mathrm{mg}, 0.750 \mathrm{mmol})$, toluene $(1.3 \mathrm{~mL})$, EtOH $(1.3 \mathrm{~mL})$, and $\mathrm{H}_{2} \mathrm{O}$ $(0.16 \mathrm{~mL})$. The mixture was stirred at $100{ }^{\circ} \mathrm{C}$ under Ar for $24 \mathrm{~h}$. The resulting solution was poured into brine, and the aqueous layer was extracted with EtOAc. The combined organic layer was washed with brine, dried over $\mathrm{Na}_{2} \mathrm{SO}_{4}$, and concentrated. The residue was purified by a PTLC ( $n$ hexane $/$ EtOAc $=4: 1)$ to give $\mathbf{1 3 b}(21.7 \mathrm{mg}, 0.0780 \mathrm{mmol}, 39 \%$ yield $)$ as a yellow oil.

\section{1-(2-Benzylphenyl)-3,5-dimethyl-1H-pyrazole (13b, Scheme 2)}<smiles>Cc1cc(C)n(-c2ccccc2Cc2ccccc2)n1</smiles>

${ }^{1} \mathrm{H}$ NMR $\left(\mathrm{CDCl}_{3}, 400 \mathrm{MHz}\right) \delta$ 7.38-7.32 (m, 1H), 7.32-7.24 (m, 2H), 7.22-7.09 (m, 4H), $6.94(\mathrm{~d}$, $J=6.9 \mathrm{~Hz}, 2 \mathrm{H}), 5.88(\mathrm{~s}, 1 \mathrm{H}), 3.80(\mathrm{~s}, 2 \mathrm{H}), 2.31(\mathrm{~s}, 3 \mathrm{H}), 1.77(\mathrm{~s}, 3 \mathrm{H}) ;{ }^{13} \mathrm{C} \mathrm{NMR}\left(\mathrm{CDCl}_{3}, 100 \mathrm{MHz}\right) \delta$ 148.6, 140.6, 139.9, 139.8, 138.5, 130.9, 129.1, 129.0, 128.2, 128.2, 126.9, 126.0, 105.0, 37.3, 13.6, 11.1; HRMS (ESI) m/z: [M+Na] ${ }^{+}$Calcd for $\mathrm{C}_{18} \mathrm{H}_{18} \mathrm{~N}_{2} \mathrm{Na} 285.1362$; Found 285.1362. 
Procedure for one-pot homocoupling (Scheme 2, condition g): To a Schlenk flask were added the crude residue, $\mathrm{CuF}_{2}(60.9 \mathrm{mg}, 0.600 \mathrm{mmol}), 1$,4-dioxane $(2.4 \mathrm{~mL}), \mathrm{MeOH}(1.2 \mathrm{~mL})$, and $\mathrm{H}_{2} \mathrm{O}(1.2$ $\mathrm{mL})$. The mixture was stirred at $100{ }^{\circ} \mathrm{C}$ under air for $24 \mathrm{~h}$. The reaction was quenched with water, and the aqueous layer was extracted with EtOAc. The combined organic layer was washed with brine, dried over $\mathrm{Na}_{2} \mathrm{SO}_{4}$, and concentrated. The residue was purified by PTLC (dichloromethane/EtOAc $=$ 10:1) to give 14b (30.8 $\mathrm{mg}, 0.0898 \mathrm{mmol}, 90 \%$ yield) as a brown solid.

\section{2,2'-Bis(3,5-dimethyl-1H-pyrazol-1-yl)-1,1'-biphenyl (14b, Scheme 2)}<smiles>Cc1cc(C)n(-c2ccccc2-c2ccccc2-n2nc(C)cc2C)n1</smiles>

Mp. $120.5^{\circ} \mathrm{C}$ (decomposition); ${ }^{1} \mathrm{H}$ NMR $\left(\mathrm{CDCl}_{3}, 400 \mathrm{MHz}\right) \delta 7.36-7.19(\mathrm{~m}, 6 \mathrm{H}), 7.03(\mathrm{~d}, J=7.5$ $\mathrm{Hz}, 2 \mathrm{H}), 5.80(\mathrm{~s}, 2 \mathrm{H}), 2.13(\mathrm{~s}, 6 \mathrm{H}), 2.00(\mathrm{~s}, 6 \mathrm{H}) ;{ }^{13} \mathrm{C} \mathrm{NMR}\left(\mathrm{CDCl}_{3}, 100 \mathrm{MHz}\right) \delta 148.3,140.9,137.8$, 135.8, 131.1, 128.2, 127.9, 127.9, 105.6, 13.5, 11.8; HRMS (ESI) $\mathrm{m} / \mathrm{z}:[\mathrm{M}+\mathrm{Na}]^{+}$Calcd for $\mathrm{C}_{22} \mathrm{H}_{22} \mathrm{~N}_{4} \mathrm{Na} 365.1737$; Found 365.1744. 


\section{Control Experiments}

Procedure for $\mathbf{C}-\mathbf{H}$ borylation with additives (Scheme 3a): $\left[\mathrm{Cp}^{\mathrm{A} 3} \mathrm{RhCl}_{2}\right]_{2}(3.0 \mathrm{mg}, 0.0025$ $\mathrm{mmol}), \mathrm{NaO}^{t} \mathrm{Bu}(1.0 \mathrm{mg}, 0.010 \mathrm{mmol})$ and THF $(0.4 \mathrm{~mL})$ were placed in $3 \mathrm{~mL}$ screw cap vial and the mixture was stirred at room temperature for $10 \mathrm{~min}$. After stirring, to this mixture were added 2 (50.8 $\mathrm{mg}, 0.200 \mathrm{mmol}),\left[\operatorname{Ir}(\mathrm{dFppy})_{3}\right](0.8 \mathrm{mg}, 0.0010 \mathrm{mmol}), \mathbf{1 a}(13.2 \mu \mathrm{L}, 0.100 \mathrm{mmol}), \mathbf{1 5}(10.3 \mu \mathrm{L}, 0.100$ $\mathrm{mmol})$ or $16(15.6 \mathrm{mg}, 0.100 \mathrm{mmol})$ or $17(18.0 \mathrm{mg}, 0.100 \mathrm{mmol})$, and THF $(0.6 \mathrm{~mL})$ in this order. The vial was capped and wrapped with a Teflon seal. The mixture was stirred at room temperature under Ar with blue LEDs irradiation for $18 \mathrm{~h}$. The resulting mixture was concentrated under reduced pressure. To the residue were added 1,3,5-trimethylbenzene (as an internal standard) and $\mathrm{CDCl}_{3}$. Then NMR yield was analyzed by ${ }^{1} \mathrm{H}$ NMR.

Procedure for $\mathbf{C}-\mathbf{H}$ borylation under air (Scheme 3a): The borylation reactions were carried out according to the general procedure for Scheme 1 using $\left[\mathrm{Cp}^{\mathrm{A} 3} \mathrm{RhCl}_{2}\right]_{2}(3.0 \mathrm{mg}, 0.0025 \mathrm{mmol})$, $\mathrm{NaO}^{t} \mathrm{Bu}(1.0 \mathrm{mg}, 0.010 \mathrm{mmol}),\left[\operatorname{Ir}(\mathrm{dFppy})_{3}\right](0.8 \mathrm{mg}, 0.0010 \mathrm{mmol}), 2(50.8 \mathrm{mg}, 0.200 \mathrm{mmol}), \mathbf{1 a}$ $(13.2 \mu \mathrm{L}, 0.100 \mathrm{mmol})$, and THF $(1.0 \mathrm{~mL})$ at room temperature under air with blue LEDs irradiation for $18 \mathrm{~h}$. Then NMR yield was analyzed by ${ }^{1} \mathrm{H}$ NMR. 


\section{Synthesis of $\mathrm{Cp}^{\mathrm{A} 3} \mathrm{Rh}(\operatorname{cod})$ Complex and $\mathrm{Rh}(\mathrm{I})$ Catalyzed Experiments}

2-\{4,6-Diphenyl-3,5-dihydro-1H-cyclopenta[c]furan-5-yl $\}-N$-(4-methoxyphenyl)acetamide (Scheme 3b)

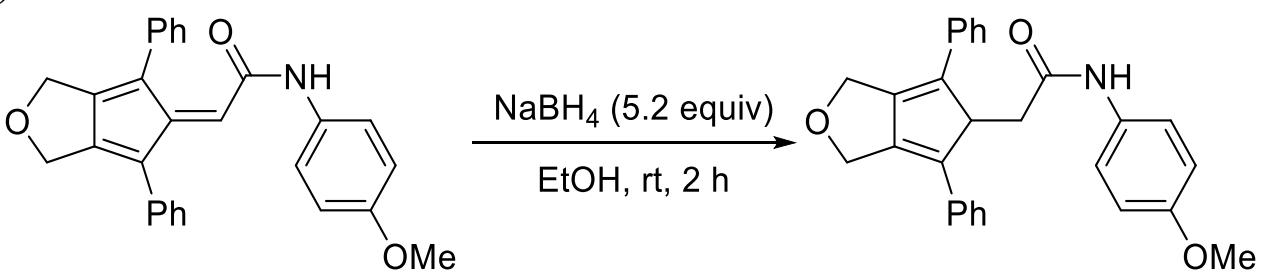

To a solution of 2-\{4,6-diphenyl- $1 H$-cyclopenta $[c]$ furan-5(3H)-ylidene $\}-N$-(4methoxyphenyl)acetamide $3(210.8 \mathrm{mg}, 0.500 \mathrm{mmol})$ in EtOH $(5 \mathrm{~mL})$ was added $\mathrm{NaBH}_{4}(98.4 \mathrm{mg}$, $2.60 \mathrm{mmol}$ ) at $0{ }^{\circ} \mathrm{C}$, and the mixture was stirred at room temperature under Ar for $2 \mathrm{~h}$. The reaction was quenched with saturated aqueous $\mathrm{NH}_{4} \mathrm{Cl}$ and extracted with EtOAc. The organic layer was washed with brine, dried over $\mathrm{Na}_{2} \mathrm{SO}_{4}$, and concentrated. The residue was diluted with $\mathrm{CH}_{2} \mathrm{Cl}_{2}(10$ $\mathrm{mL})$ and poured into $n$-hexane $(100 \mathrm{~mL})$, and the resulting precipitates were collected, washed with a $n$-hexane/ $\mathrm{Et}_{2} \mathrm{O}$ solution ( $n$-hexane/ $\left.\mathrm{Et}_{2} \mathrm{O}=3: 1\right)(10 \mathrm{~mL})$ three times, and dried under vacuum to give the title compound (139.2 $\mathrm{mg}, 0.329 \mathrm{mmol}, 66 \%$ yield) as a colorless solid.

Mp. 198.2 ${ }^{\circ} \mathrm{C}$ (decomposition); ${ }^{1} \mathrm{H} \mathrm{NMR}\left(\mathrm{CDCl}_{3}, 400 \mathrm{MHz}\right) \delta 7.37(\mathrm{dd}, J=8.0,7.3 \mathrm{~Hz}, 4 \mathrm{H}), 7.33-$ $7.28(\mathrm{~m}, 4 \mathrm{H}), 7.24-7.18(\mathrm{~m}, 2 \mathrm{H}), 7.10-7.04(\mathrm{~m}, 2 \mathrm{H}), 6.81-6.75(\mathrm{~m}, 2 \mathrm{H}), 6.63(\mathrm{br}, 1 \mathrm{H}), 5.11-5.03(\mathrm{~m}$, $1 \mathrm{H}), 4.96(\mathrm{dd}, J=14.1,1.9 \mathrm{~Hz}, 2 \mathrm{H}), 4.74(\mathrm{dd}, J=14.5,2.5 \mathrm{~Hz}, 2 \mathrm{H}), 3.77$ (s, 3H), $2.73(\mathrm{~d}, J=5.5 \mathrm{~Hz}$, $2 \mathrm{H}) ;{ }^{13} \mathrm{C} \mathrm{NMR}\left(\mathrm{CDCl}_{3}, 100 \mathrm{~Hz}\right) \delta 168.8,156.5,146.9,136.7,134.2,130.4,129.1,126.9,126.8,122.0$, 114.0, 66.7, 55.5, 55.1, 39.3; HRMS (ESI) m/z: [M+Na] ${ }^{+}$Calcd for $\mathrm{C}_{28} \mathrm{H}_{25} \mathrm{NNaO}_{3}$ 446.1727; Found 446.1723 .

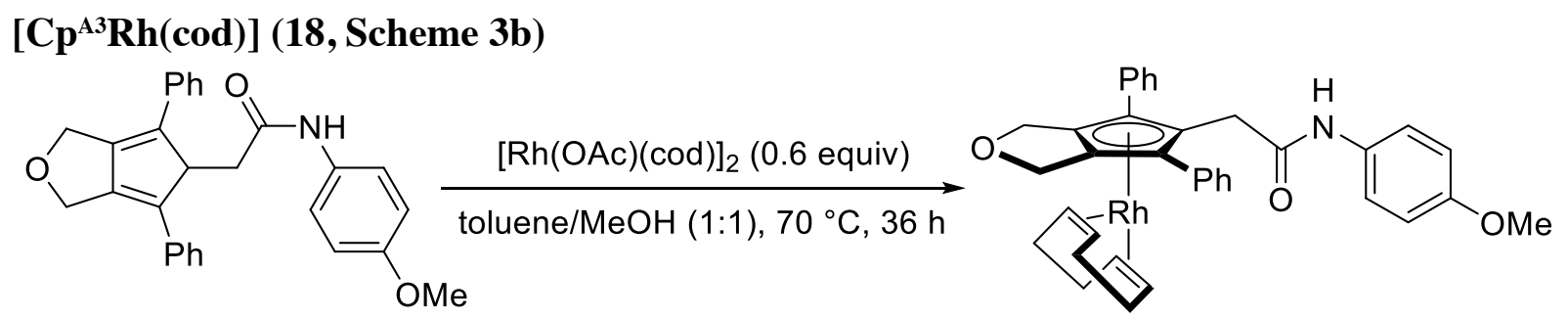

To a Schlenk flask were added 2-\{4,6-diphenyl-3,5-dihydro- $1 H$-cyclopenta[ $[c]$ furan-5-yl $\}-N$-(4methoxyphenyl)acetamide $(84.7 \mathrm{mg}, 0.200 \mathrm{mmol}),[\mathrm{Rh}(\mathrm{OAc})(\mathrm{cod})]_{2}{ }^{39}(64.8 \mathrm{mg}, 0.120 \mathrm{mmol})$, toluene $(1 \mathrm{~mL})$, and $\mathrm{MeOH}(1 \mathrm{~mL})$ in this order. The mixture was stirred at $70{ }^{\circ} \mathrm{C}$ for $36 \mathrm{~h}$. The resulting mixture was concentrated under reduced pressure, and the residue was purified by a silica gel column chromatography $(n$-hexane/EtOAc/dichloromethane $=1: 1: 1)$ to give $\mathbf{1 8}(104.6 \mathrm{mg}, 0.165$ mmol, $83 \%$ yield) as a yellow solid.

Mp. $185.4{ }^{\circ} \mathrm{C}$ (decomposition); ${ }^{1} \mathrm{H} \mathrm{NMR}\left(\mathrm{CDCl}_{3}, 400 \mathrm{MHz}\right) \delta 7.62-7.59(\mathrm{~m}, 4 \mathrm{H}), 7.41-7.33$ (m, 5H), 7.31-7.24 (m, 2H), 7.15-7.09 (m, 2H), 6.83-6.77 (m, 2H), $4.78(\mathrm{~d}, J=10.3 \mathrm{~Hz}, 2 \mathrm{H}), 4.69(\mathrm{~d}, J$ $=10.3 \mathrm{~Hz}, 2 \mathrm{H}), 3.77(\mathrm{~s}, 3 \mathrm{H}), 3.49(\mathrm{br}, 4 \mathrm{H}), 3.28(\mathrm{~s}, 2 \mathrm{H}), 2.32-2.21(\mathrm{~m}, 4 \mathrm{H}), 2.02-1.92(\mathrm{~m}, 4 \mathrm{H}) ;{ }^{13} \mathrm{C}$ $\mathrm{NMR}\left(\mathrm{CDCl}_{3}, 100 \mathrm{MHz}\right) \delta 168.6,156.8,134.1,130.4,129.1,128.8,127.0,122.5,114.1,108.1,108.1$, 100.3, 95.3, 73.4, 73.2, 66.7, 55.5, 34.6, 32.1; HRMS (ESI) m/z: [M+Na] ${ }^{+}$Calcd for $\mathrm{C}_{36} \mathrm{H}_{36} \mathrm{NNaO}_{3} \mathrm{Rh}$ 656.1642; Found 656.1643.

Procedure for $\mathrm{Cp}^{\mathrm{A} 3} \mathbf{R h}(\operatorname{cod})$ catalyzed $\mathrm{C}-\mathrm{H}$ borylation (Figure $3 \mathrm{~b}$ ): The borylation reactions were carried out according to the general procedure for Scheme 1 using [C $\left.\mathrm{Cp}^{\mathrm{A} 3} \mathrm{Rh}(\mathrm{cod})\right](3.2 \mathrm{mg}$, $0.0050 \mathrm{mmol}), \mathrm{NaO}^{t} \mathrm{Bu}(1.0 \mathrm{mg}, 0.010 \mathrm{mmol}),\left[\operatorname{Ir}(\mathrm{dFppy})_{3}\right](0.8 \mathrm{mg}, 0.0010 \mathrm{mmol}), 2(50.8 \mathrm{mg}$, $0.200 \mathrm{mmol}), \mathbf{1 a}(13.2 \mu \mathrm{L}, 0.100 \mathrm{mmol})$, and THF $(1.0 \mathrm{~mL})$ at room temperature under visible light irradiation or without irradiation for $18 \mathrm{~h}$. Then NMR yield was analyzed by ${ }^{1} \mathrm{H}$ NMR. 


\section{UV-vis Absorption Study}

\section{[2-(1H-Pyrazol-1-yl)phenyl](pentamethylcyclopentadienyl)rhodium(III) acetate (A1) ${ }^{40}$}

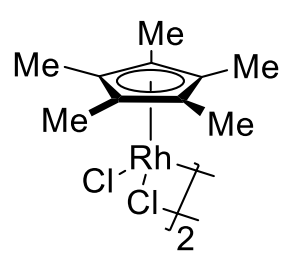

$\left[\mathrm{Cp}^{*} \mathrm{RhCl}_{2}\right]_{2}$

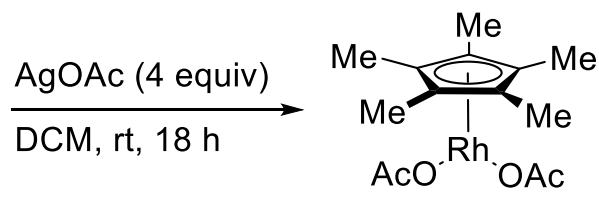

$\mathrm{Cp}^{*} \mathrm{Rh}(\mathrm{OAc})_{2}$<smiles>c1ccc(-n2cccn2)cc1</smiles>

(1.5 equiv)

$\mathrm{MeOH}, \mathrm{rt}, 48 \mathrm{~h}$

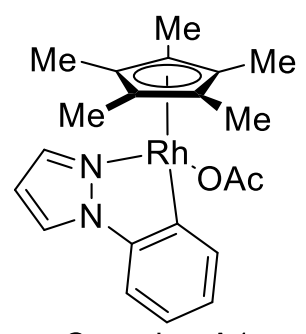

Complex A1

$\left[\mathrm{Cp} * \mathrm{RhCl}_{2}\right]_{2}(61.8 \mathrm{mg}, 0.100 \mathrm{mmol})$ and $\mathrm{AgOAc}(66.8 \mathrm{mg}, 0.400 \mathrm{mmol})$ were stirred in dichloromethane $(7 \mathrm{~mL})$ overnight at room temperature. Precipitate formation occurred upon trituration/sonification in $n$-hexane/EtOAc solution. The solid was filtered and washed with $n$-hexane to give the $\mathrm{Cp} * \mathrm{Rh}(\mathrm{OAc})_{2}$ intermediate as an orange solid (40.0 mg, $56 \%$ ). Then, 1-phenylpyrazole $(8.0 \mathrm{mg}, 0.055 \mathrm{mmol}), \mathrm{Cp} * \mathrm{Rh}(\mathrm{OAc})_{2}(18.0 \mathrm{mg}, 0.0505 \mathrm{mmol})$, and dry $\mathrm{MeOH}(1.0 \mathrm{~mL})$ were added to a vial under argon atmosphere. The vial was sealed, and the reaction mixture was stirred overnight at room temperature. The solvent was removed under reduced pressure. Precipitate formation occurred upon trituration/sonification in $n$-hexane/ EtOAc solution. The solid was filtered and washed with $n$-hexane to give the complex A1 as an orange solid $(7.3 \mathrm{mg}, 16 \%)$.

${ }^{1} \mathrm{H}$ NMR $\left(400 \mathrm{MHz}, \mathrm{CDCl}_{3}\right) \delta 8.06-8.01(\mathrm{~m}, 2 \mathrm{H}), 7.91(\mathrm{~d}, J=2.6 \mathrm{~Hz}, 1 \mathrm{H}), 7.16(\mathrm{~m}, 2 \mathrm{H}), 7.07$ (ddd, $J=8.1,6.9,1.2 \mathrm{~Hz}, 1 \mathrm{H}), 6.49(\mathrm{~d}, J=2.4,2.4 \mathrm{~Hz}, 1 \mathrm{H}), 1.69(\mathrm{~s}, 3 \mathrm{H}), 1.65(\mathrm{~s}, 15 \mathrm{H}) ;{ }^{13} \mathrm{C}$ NMR $\left(100 \mathrm{MHz}, \mathrm{CDCl}_{3}\right) \delta 177.3\left(\mathrm{C}_{\mathrm{q}}\right), 160.4\left(\mathrm{~d},{ }^{1} J_{\mathrm{C}-\mathrm{Rh}}=34.4 \mathrm{~Hz}, \mathrm{C}_{\mathrm{q}}\right), 142.7\left(\mathrm{C}_{\mathrm{q}}\right), 140.8(\mathrm{CH}), 137.6(\mathrm{CH})$, $126.7(\mathrm{CH}), 124.4(\mathrm{CH}), 123.5(\mathrm{CH}), 110.8(\mathrm{CH}), 107.7(\mathrm{CH}), 94.7\left(\mathrm{~d},{ }^{1} J_{\mathrm{C}-\mathrm{Rh}}=6.9 \mathrm{~Hz}, \mathrm{Cq}\right), 24.8(\mathrm{~d}$, $\left.{ }^{1} J_{\mathrm{C}-\mathrm{Rh}}=34.4 \mathrm{~Hz}, \mathrm{CH}_{3}\right), 9.5\left(\mathrm{CH}_{3}\right)$; HRMS (ESI) m/z: [M-OAc] ${ }^{+} \mathrm{Calcd}$ for $\mathrm{C}_{19} \mathrm{H}_{22} \mathrm{~N}_{2} \mathrm{Rh} 381.0833$; Found: 381.0835 .

\section{[2-(2-pyridinyl)phenyl](pentamethylcyclopentadienyl)rhodium(III) acetate (A3) ${ }^{41}$}

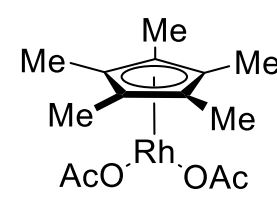

$C p^{*} \operatorname{Rh}(\mathrm{OAc})_{2}$

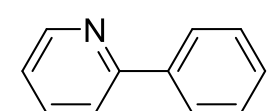

(1.5 equiv)

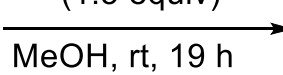

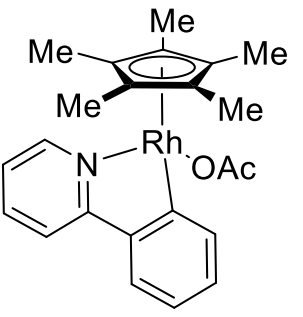

Complex A3

2-phenylpyridine $(7.8 \mathrm{mg}, 0.050 \mathrm{mmol})$, above synthesized $\mathrm{Cp} * \mathrm{Rh}(\mathrm{OAc})_{2}$ intermediate $(10.7 \mathrm{mg}$, $0.0300 \mathrm{mmol})$, and dry $\mathrm{MeOH}(1.0 \mathrm{~mL})$ were added to a vial under argon atmosphere. The vial was sealed, and the reaction mixture was stirred overnight at room temperature. The solvent was removed under reduced pressure. Precipitate formation occurred upon trituration/sonification in $n$-hexane/ EtOAc solution. The solid was filtered and washed with $n$-hexane to give the complex A3 as an orange solid $(2.7 \mathrm{mg}, 20 \%)$.

Mp. $112.3{ }^{\circ} \mathrm{C}$ (decomposition); ${ }^{1} \mathrm{H}$ NMR $\left(\mathrm{CDCl}_{3}, 400 \mathrm{MHz}\right) \delta 9.23(\mathrm{~d}, J=5.5 \mathrm{~Hz}, 1 \mathrm{H}), 8.08(\mathrm{~d}, J$ $=7.5,1 \mathrm{H}), 7.75-7.65(\mathrm{~m}, 2 \mathrm{H}), 7.60(\mathrm{dd}, J=7.6,0.8 \mathrm{~Hz}, 1 \mathrm{H}), 7.30-7.24(\mathrm{~m}, 1 \mathrm{H}), 7.16-7.12(\mathrm{~m}, 1 \mathrm{H})$, $7.08(\mathrm{t}, J=7.3,1 \mathrm{H}), 1.70(\mathrm{~s}, 3 \mathrm{H}), 1.59(\mathrm{~s}, 15 \mathrm{H}) ;{ }^{13} \mathrm{C}$ NMR $\left(\mathrm{CDCl}_{3}, 100 \mathrm{MHz}\right) \delta 179.9(\mathrm{~d}, J=33.7$ Hz), 177.8, 165.7 (d, $J=1.6 \mathrm{~Hz}), 153.2,144.4,137.0,136.3,130.2,123.2,122.9,121.3,118.2(\mathrm{~d}, J$ $=1.3 \mathrm{~Hz}), 94.9(\mathrm{~d}, J=6.5 \mathrm{~Hz}), 24.9(\mathrm{~d}, J=1.8 \mathrm{~Hz}), 9.4$; HRMS (ESI) m/z: [M-OAc] ${ }^{+}$Calcd for $\mathrm{C}_{21} \mathrm{H}_{23} \mathrm{NRh}$ 392.0880; Found 392.0884. 
Figure S2. Absorption spectrum of Rh complexes A1 and A3 in $\mathrm{CHCl}_{3}\left(1.0 \times 10^{-5} \mathrm{M}\right)$.

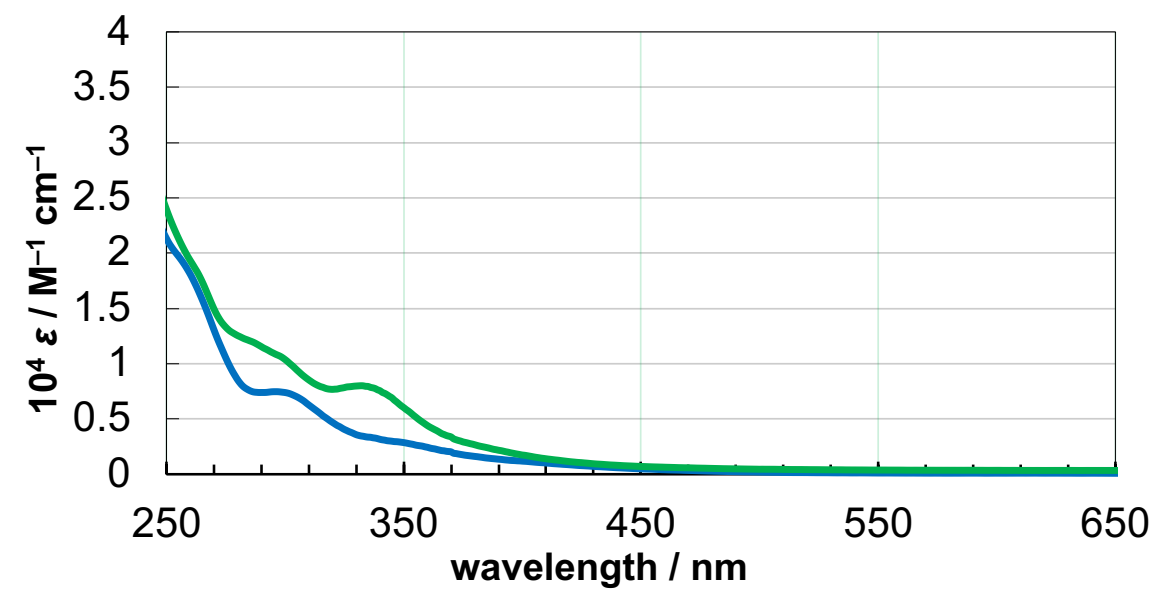

A1: Blue

A3: Green 


\section{Computational Study}

All calculations were carried with the Gaussian 16 program package. ${ }^{42}$ The hybrid density functional method based on M06 ${ }^{43}$ with a standard 6-31g* basis set (LANL2DZ basis set for Rh) was used for geometry optimizations. The $6-311+\mathrm{g}^{* *}$ basis set (SDD basis set for Rh) was used to calculate the single-point energies because it was envisaged that this strategy would provide greater accuracy with regard to the energetic information. The solvation effect was examined by performing single-point self-consistent reaction field (SCRF) calculations ${ }^{44}$ based on the polarizable continuum model (PCM) for gas-phase optimized structures. Geometry optimization and vibrational analysis were performed at the same level. All stationary points were optimized without any symmetry assumptions, and characterized by normal coordinate analysis at the same level of theory (number of imaginary frequencies, NIMAG, 0 for minima and 1 for TSs). The intrinsic reaction coordinate (IRC) method was used to track minimum energy paths from transition structures to the corresponding local minima.${ }^{45}$ Excitation wavelengths and oscillator strengths were obtained at the density functional level using time-dependent perturbation theory (TD-DFT) approach.

Table S1. The Gibbs-free energies and imaginary frequencies for Figure 2.

\begin{tabular}{|c|c|c|c|}
\hline & $\begin{array}{l}G \text { (hartree)@M06/6-311++g** } \\
\& \text { SDD +PCM [THF] }\end{array}$ & $\begin{array}{l}G \text { (hartree)@M06/6-31g* } \\
\& \text { LANL2DZ }\end{array}$ & $\begin{array}{l}\text { imaginary } \\
\text { frequency }\left(\mathrm{cm}^{-1}\right)\end{array}$ \\
\hline$B_{2}(\mathbf{e g})_{2}$ & -507.756923 & -507.613820 & \\
\hline HB(eg) & -254.474635 & -228.923053 & \\
\hline $\mathbf{N a H}$ & -162.897159 & -162.885853 & \\
\hline NaOMe & -277.417583 & -277.379956 & \\
\hline МeOH & -115.664594 & -115.629596 & \\
\hline MeO-B(eg) & -368.999126 & -368.894212 & \\
\hline$(\mathrm{MeO})(\mathrm{eg}) \mathrm{B}-\mathrm{B}(\mathrm{eg})$ & & -622.620784 & \\
\hline radical anion & -622.793846 & & \\
\hline$(\mathrm{MeO})(\mathrm{eg}) \mathrm{B}-\mathrm{B}(\mathrm{eg})$ anion & -622.939302 & -622.684758 & \\
\hline $\mathrm{Cp}^{\mathrm{A} 3} \mathrm{Rh}(\mathrm{III})(\mathrm{OMe})_{2}$ & -1701.504316 & -1699.989514 & \\
\hline $\mathrm{Cp}^{\mathrm{A} 3} \mathrm{Rh}(\mathrm{II})(\mathrm{OMe})_{2}$ anion & -1701.625457 & -1700.050429 & \\
\hline $\mathrm{Cp}^{\mathrm{A} 3} \mathbf{R h}(\mathrm{II})(\mathrm{OMe})$ & -1586.438365 & -1584.964736 & \\
\hline $\mathrm{Cp}^{\mathrm{A} 3} \mathrm{Rh}(\mathrm{I})(\mathrm{OMe})$ anion & -1586.536744 & -1585.004216 & \\
\hline INT1 & -1950.338342 & -1948.780432 & \\
\hline TS1 & -1950.340859 & -1948.778046 & -501.00 \\
\hline INT2 & -1950.349446 & -1948.786808 & \\
\hline INT1' & -2180.443249 & -2178.806346 & \\
\hline TS1' & -2180.391978 & -2178.754664 & -498.65 \\
\hline INT2' & -2180.47486 & -2178.835003 & \\
\hline INT3' & -2064.813062 & -2063.215211 & \\
\hline INT3-Rh(III) & -2458.100839 & -2456.396295 & \\
\hline TS2-Rh(III) & -2458.036679 & -2456.335181 & -811.52 \\
\hline INT4-Rh(III) & -2458.109115 & -2456.411275 & \\
\hline INT3-Rh(IV) & -2457.368052 & -2456.193194 & \\
\hline TS2-Rh(IV) & -2457.318016 & -2456.146396 & -261.99 \\
\hline INT4-Rh(IV) & -2457.410621 & -2456.246168 & \\
\hline INT3-Rh(II) & -2457.623093 & -2456.420782 & \\
\hline TS2A-Rh(II) & -2457.606952 & -2456.393419 & -58.21 \\
\hline INT3A-Rh(II) & -2457.607389 & -2456.393783 & \\
\hline
\end{tabular}




\begin{tabular}{|c|c|c|c|}
\hline INT3B-Rh(II) & -2457.608713 & -2456.398025 & \\
\hline TS2B-Rh(II) & -2457.606127 & -2456.396988 & -38.36 \\
\hline INT4-Rh(II) & -2457.644084 & -2456.447588 & \\
\hline INT5 & -2203.651757 & -2202.024934 & \\
\hline TS3 & -2203.636838 & -2202.014659 & -66.02 \\
\hline INT6 & -2203.646445 & -2202.026153 & \\
\hline INT2-Rh(II) & -1950.425419 & -1948.807632 & \\
\hline${ }^{3}$ INT2* & -1950.274451 & -1948.708854 & \\
\hline${ }^{3}$ INT3* & -2458.024092 & -2456.328503 & \\
\hline${ }^{3} \mathbf{I N T} 4 *$ & -2458.038255 & -2456.343166 & \\
\hline $\operatorname{Ir}(\mathrm{III})(\mathrm{dFppy})_{3}$ & -2135.020159 & -2134.773401 & \\
\hline${ }^{3} \operatorname{Ir}(\mathrm{III})(\mathbf{d F p p y})_{3} *$ & -2134.913824 & -2134.67674 & \\
\hline $\operatorname{Ir}(\mathbf{I I})(\mathbf{d F p p y})_{3}$ & -2135.102137 & -2134.797806 & \\
\hline
\end{tabular}

Figure S3. Chemical structures of TS2-Rh(III), TS2-Rh(II), and TS2A-Rh(II)
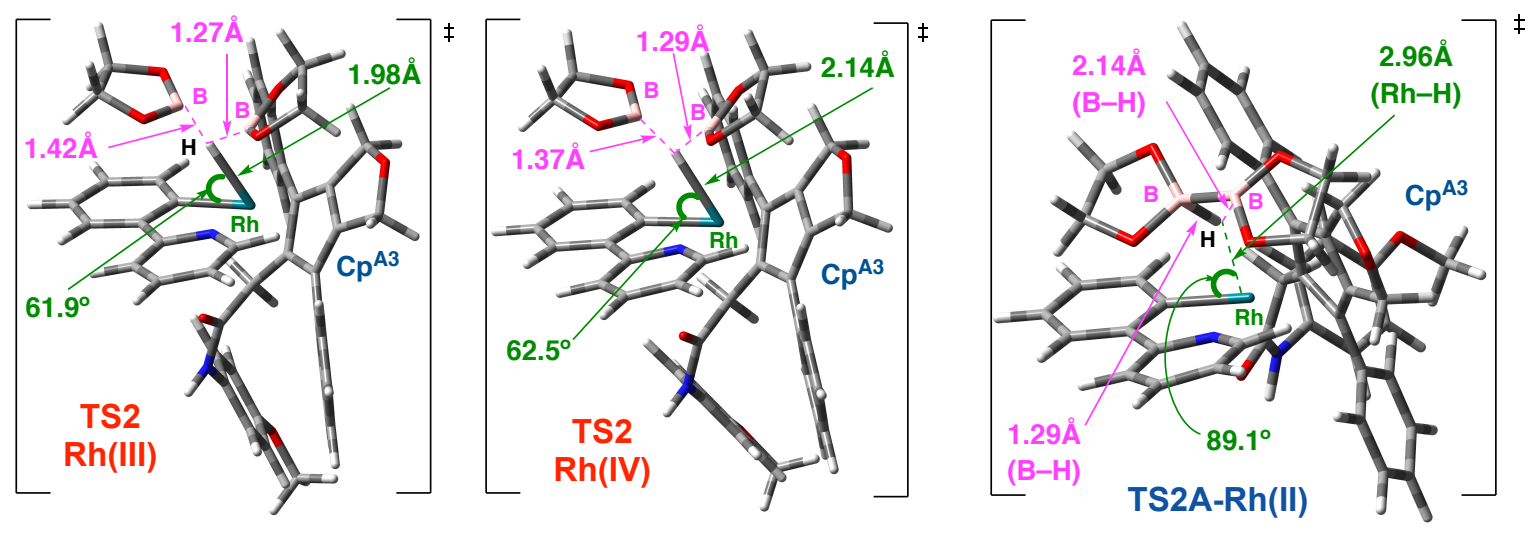
Figure S4. TD-DFT calculations for theoretical UV/vis absorption spectrum and molecular orbitals contributing dominant transitions of Rh(III) complexes.

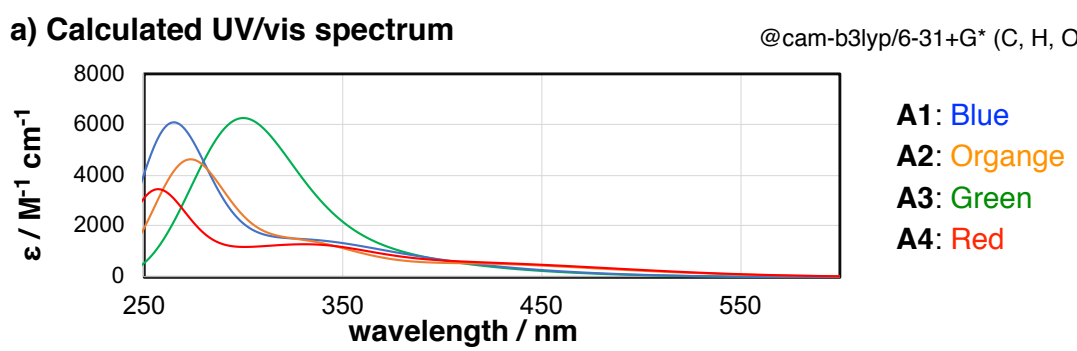

b) Comparision of molecular orbitals contributing dominant transitions

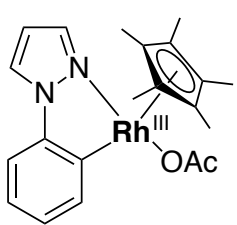

A1

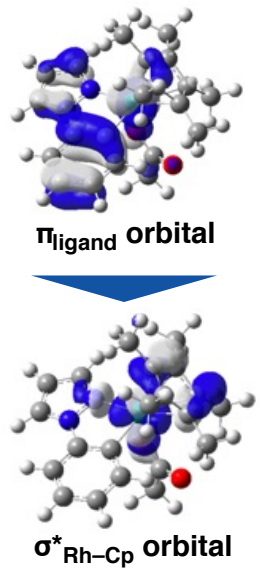

ligand-to-metal CT transition (452 $\mathrm{nm})$

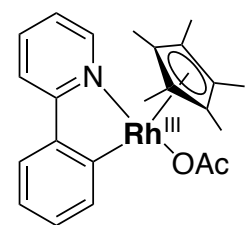

A3

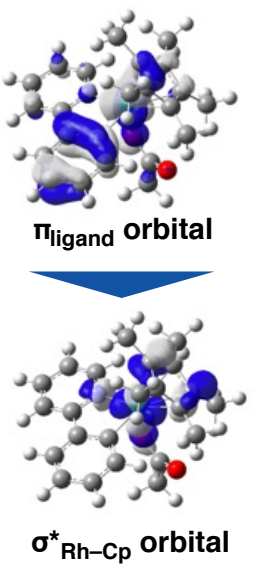

ligand-to-metal CT transition (457 $\mathrm{nm})$

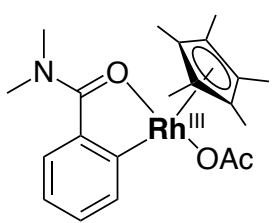

A2

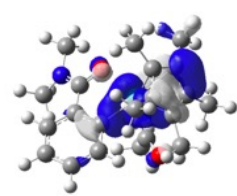

$\sigma_{\mathrm{Rh}-\mathrm{OAc}}$ orbital

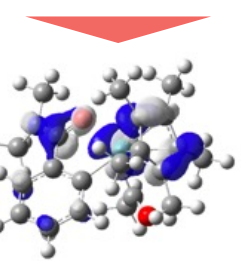

Rh $4 d^{*}$ orbital

metal-centered transition

$(481 \mathrm{~nm})$

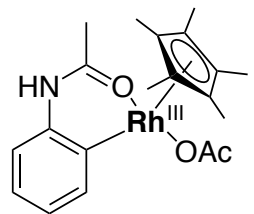

A4
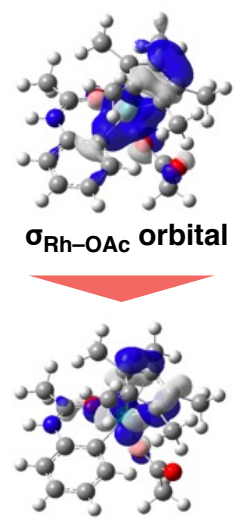

Rh 4d* orbital

metal-centered transition

(474 nm)

Table S2. TD-DFT vertical one-electron excitations of A1-A4 (Figure S4).

\begin{tabular}{|c|ccccc|}
\hline Compound & $\begin{array}{c}\text { excited } \\
\text { state }\end{array}$ & $\begin{array}{c}\text { Energy } \\
(\mathrm{eV})\end{array}$ & $\begin{array}{c}\text { Wavelength } \\
(\mathrm{nm})\end{array}$ & $\begin{array}{c}\text { oscillator } \\
\text { strength }(f)\end{array}$ & $\begin{array}{c}\text { description } \\
\text { [Relative major contribution on the excited state by } \\
\text { blue light (445-455 nm) is listed.] }\end{array}$ \\
\hline $\mathrm{A} 1$ & 1 & 2.7425 & 452.09 & 0.0029 & HOMO $\rightarrow$ LUMO (0.61348, LMCT) \\
& 2 & 3.1337 & 395.64 & 0.0091 & \\
& 3 & 3.4857 & 355.70 & 0.0139 & \\
& 4 & 3.7322 & 332.20 & 0.0162 & \\
& 5 & 4.0357 & 307.22 & 0.0162 & \\
& 6 & 4.3866 & 282.64 & 0.0031 & \\
& 7 & 4.4780 & 276.87 & 0.0041 & \\
& 8 & 4.5521 & 272.37 & 0.0695 & \\
& 9 & 4.7477 & 261.14 & 0.0822 & \\
\hline $\mathbf{A 3}$ & 10 & 4.9337 & 251.30 & 0.0004 & \\
& 1 & 2.7151 & 456.65 & 0.0022 & HOMO $\rightarrow$ LUMO +2 (0.61749, LMCT) \\
& 2 & 3.1402 & 394.83 & 0.0089 & \\
& 3 & 3.4676 & 357.55 & 0.0114 & \\
& 4 & 3.7357 & 331.89 & 0.0218 &
\end{tabular}




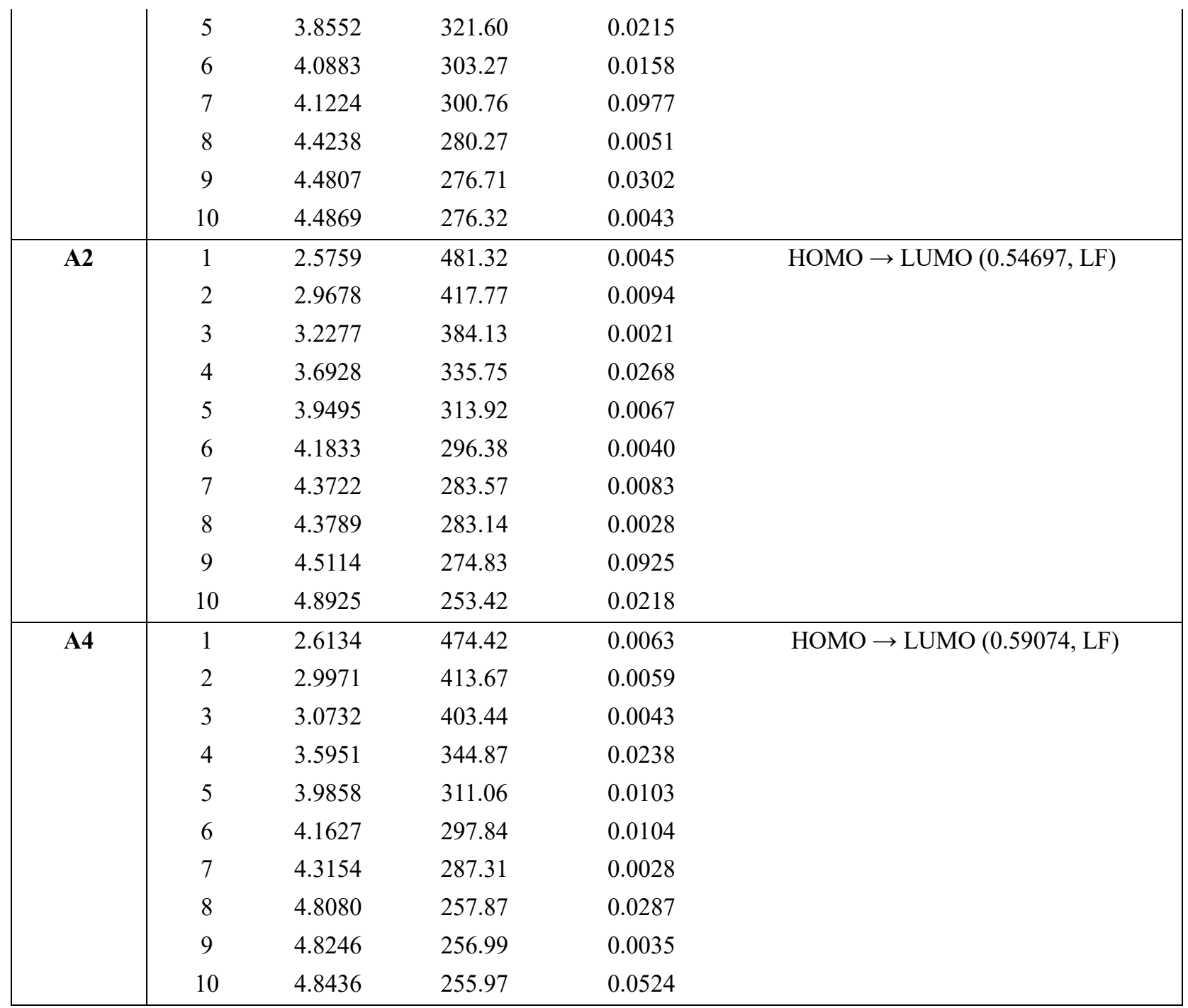

Figure S5. Molecular orbitals of A1-A4 (Figure S4).

\section{A1}

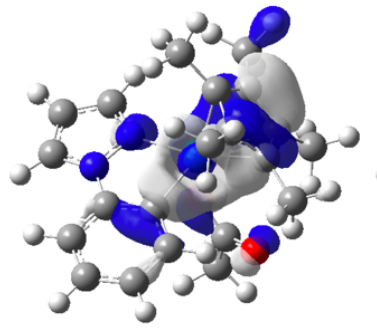

HOMO-1

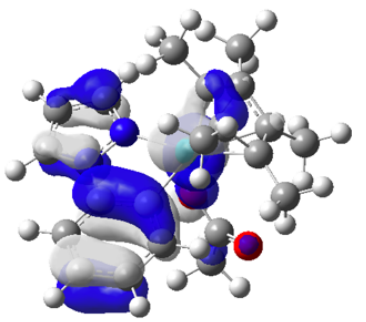

HOMO

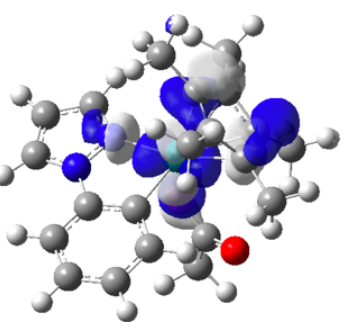

LUMO

\section{A3}

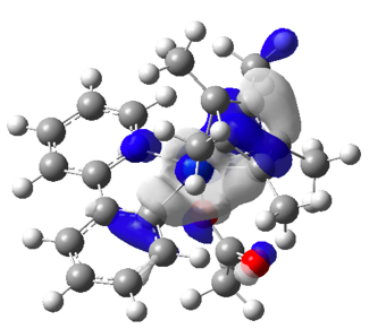

HOMO-1

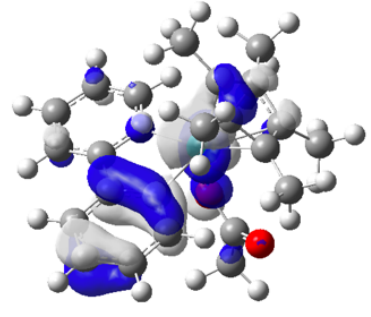

HOMO

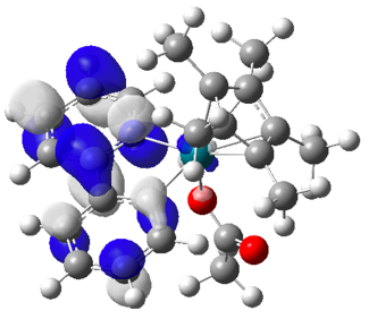

LUMO

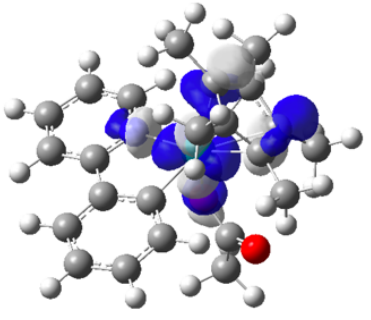

LUMO+2 


\section{A2}

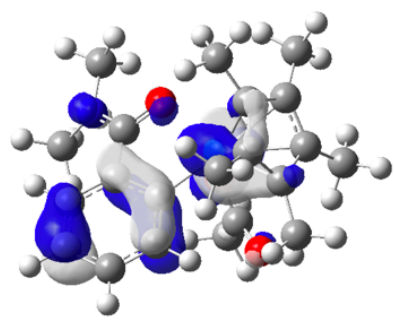

HOMO-1

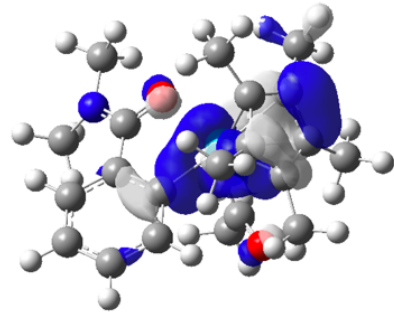

HOMO

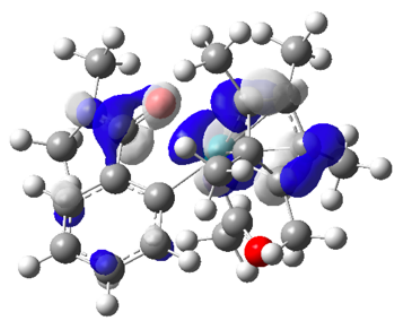

LUMO

A4

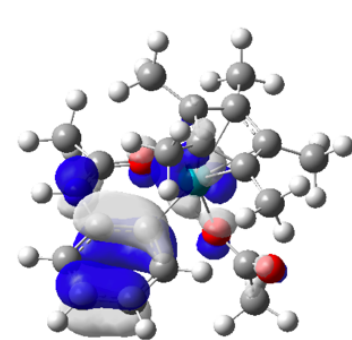

HOMO-1

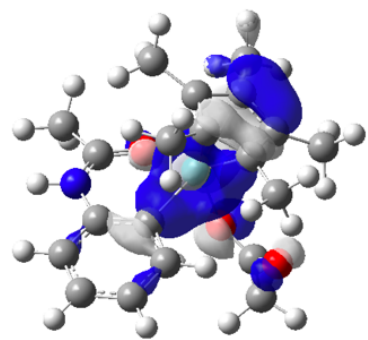

HOMO

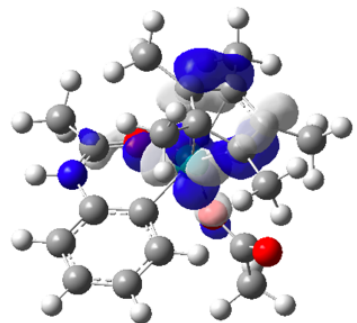

LUMO

Figure S6. Potential energy diagram from ${ }^{3}$ INT3* to ${ }^{3}$ INT4*

While ${ }^{3}$ TS2 $*$ could not be identified, we obtained the potential energy diagram by calculating some optimized structures around the transition state.

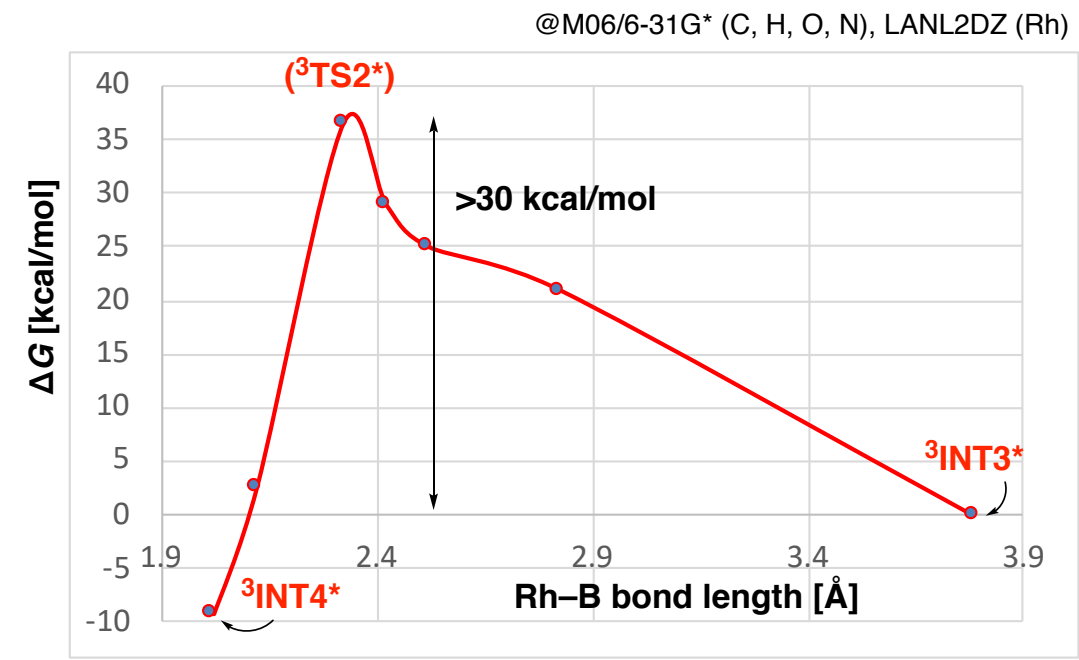


$B_{2}(\mathbf{e g})_{2}$

Sum of electronic and zero-point Energies = $-507.578902$

Sum of electronic and thermal Free Energies = $-507.613820$

$\begin{array}{lrrr}\mathrm{C} & 0.76907200 & 0.08068200 & 3.00387600 \\ \mathrm{C} & -0.76907200 & -0.08068200 & 3.00387600 \\ \mathrm{H} & 1.28472400 & -0.71138900 & 3.55461700 \\ \mathrm{H} & 1.09290900 & 1.05221200 & 3.39287100 \\ \mathrm{H} & -1.09290900 & -1.05221200 & 3.39287100 \\ \mathrm{H} & -1.28472400 & 0.71138900 & 3.55461700 \\ \mathrm{~B} & 0.00000000 & 0.00000000 & 0.85170400 \\ \mathrm{O} & 1.14561100 & 0.00000000 & 1.61313700 \\ \mathrm{O} & -1.14561100 & 0.00000000 & 1.61313700 \\ \mathrm{C} & -0.08068200 & 0.76907200 & -3.00387600 \\ \mathrm{C} & 0.08068200 & -0.76907200 & -3.00387600 \\ \mathrm{H} & -1.05221200 & 1.09290900 & -3.39287100 \\ \mathrm{H} & 0.71138900 & 1.28472400 & -3.55461700 \\ \mathrm{H} & -0.71138900 & -1.28472400 & -3.55461700 \\ \mathrm{H} & 1.05221200 & -1.09290900 & -3.39287100 \\ \mathrm{~B} & 0.00000000 & 0.00000000 & -0.85170400 \\ \mathrm{O} & 0.00000000 & -1.14561100 & -1.61313700 \\ \mathrm{O} & 0.00000000 & 1.14561100 & -1.61313700\end{array}$

\section{$\mathrm{HB}(\mathrm{eg})$}

Sum of electronic and zero-point Energies = $-254.375500$

Sum of electronic and thermal Free Energies = $-254.405010$

$\begin{array}{lrrr}\mathrm{C} & -0.89225700 & -0.76988300 & -0.01722100 \\ \mathrm{C} & -0.89142000 & 0.77084000 & 0.01729000 \\ \mathrm{H} & -1.40673400 & -1.21475100 & 0.84362600 \\ \mathrm{H} & -1.34880000 & -1.17262200 & -0.93066400 \\ \mathrm{H} & -1.34746000 & 1.17413700 & 0.93074000 \\ \mathrm{H} & -1.40547100 & 1.21621400 & -0.84354500 \\ \mathrm{O} & 0.48066700 & -1.14317400 & 0.01595600 \\ \mathrm{O} & 0.48191000 & 1.14265700 & -0.01608100 \\ \mathrm{~B} & 1.22095800 & -0.00065500 & 0.00001300 \\ \mathrm{H} & 2.40511800 & -0.00130200 & 0.00036600\end{array}$

\section{$\mathbf{N a H}$}

Sum of electronic and zero-point Energies= 162.867645

Sum of electronic and thermal Free Energies= 162.885853

$\begin{array}{cccc}\mathrm{Na} & 0.00000000 & 0.00000000 & 0.17200800 \\ \mathrm{H} & 0.00000000 & 0.00000000 & -1.89209200\end{array}$

\section{NaOMe}

Sum of electronic and zero-point Energies= 277.352645

Sum of electronic and thermal Free Energies= 277.379956

$\begin{array}{cccc}\mathrm{Na} & 1.76137100 & 0.00023700 & 0.00001300 \\ \mathrm{O} & -0.33972600 & -0.00074600 & -0.00005600 \\ \mathrm{C} & -1.69900800 & 0.00034100 & 0.00007000 \\ \mathrm{H} & -2.15423000 & -0.72773600 & 0.71899600 \\ \mathrm{H} & -2.15460700 & 0.98755700 & 0.27072200 \\ \mathrm{H} & -2.15439100 & -0.25851300 & -0.98983500\end{array}$

\section{$\mathrm{MeOH}$}

Sum of electronic and zero-point Energies= 115.606828

Sum of electronic and thermal Free Energies=

$\begin{array}{lccc}115.629596 & & & \\ \mathrm{O} & 0.74527400 & 0.12365700 & 0.00000000 \\ \mathrm{C} & -0.66316900 & -0.02050900 & 0.00000000 \\ \mathrm{H} & -1.02200200 & -0.54932800 & 0.89502700 \\ \mathrm{H} & -1.08674700 & 0.98842800 & -0.00000100 \\ \mathrm{H} & -1.02200200 & -0.54933000 & -0.89502600 \\ \mathrm{H} & 1.14757700 & -0.75596900 & 0.00000000\end{array}$

$\mathrm{MeO}-\mathrm{B}(\mathrm{eg})$

Sum of electronic and zero-point Energies= 368.862408

Sum of electronic and thermal Free Energies= 368.894212

$\begin{array}{lrrr}\mathrm{C} & 1.56697200 & 1.00562700 & -0.08532600 \\ \mathrm{C} & 1.97017500 & -0.46712800 & 0.09909900 \\ \mathrm{H} & 1.82609000 & 1.38341200 & -1.08512300 \\ \mathrm{H} & 2.02291900 & 1.67046800 & 0.65836700 \\ \mathrm{H} & 2.71840000 & -0.79840900 & -0.63136500 \\ \mathrm{H} & 2.36865200 & -0.66136000 & 1.10586900 \\ \mathrm{~B} & -0.25960100 & -0.29612200 & -0.01114100 \\ \mathrm{O} & 0.15596600 & 1.01435900 & 0.06257600 \\ \mathrm{O} & 0.77021600 & -1.19921200 & -0.07627400 \\ \mathrm{O} & -1.55207900 & -0.67799300 & -0.02012400 \\ \mathrm{C} & -2.55775600 & 0.30946400 & 0.01824700 \\ \mathrm{H} & -2.45360200 & 1.02221100 & -0.81066200 \\ \mathrm{H} & -3.52613600 & -0.19390000 & -0.06331700 \\ \mathrm{H} & -2.52748500 & 0.87317300 & 0.96038600\end{array}$

\section{(MeO)(eg)B-B(eg) anion}

Sum of electronic and zero-point Energies= 622.642702

Sum of electronic and thermal Free Energies= 622.684758

$\begin{array}{lrrr}\mathrm{C} & -2.31801900 & -1.06934900 & 1.02691700 \\ \mathrm{C} & -2.59190000 & -1.18067900 & -0.47254000 \\ \mathrm{H} & -3.23498800 & -0.82920800 & 1.60282500 \\ \mathrm{H} & -1.94489400 & -2.04478200 & 1.41403300 \\ \mathrm{H} & -3.37434000 & -0.44580500 & -0.76358300 \\ \mathrm{H} & -2.95081700 & -2.18354600 & -0.77175700 \\ \mathrm{~B} & -0.77387500 & 0.15856100 & -0.22300000 \\ \mathrm{C} & 3.20632700 & 0.35399800 & 0.15865500 \\ \mathrm{C} & 2.96459400 & -1.15290700 & 0.02361600 \\ \mathrm{H} & 3.74962800 & 0.76302800 & -0.71137500 \\ \mathrm{H} & 3.78620300 & 0.61371400 & 1.05833300 \\ \mathrm{H} & 3.59447100 & -1.61582000 & -0.75250800 \\ \mathrm{H} & 3.16567600 & -1.68601700 & 0.96962000 \\ \mathrm{~B} & 0.96018000 & -0.04608600 & -0.11940800 \\ \mathrm{O} & -1.36817300 & -0.89490800 & -1.06244100 \\ \mathrm{O} & -1.36109000 & -0.07339400 & 1.14529200 \\ \mathrm{O} & 1.91838000 & 0.91809800 & 0.22650900 \\ \mathrm{O} & 1.60465700 & -1.27193100 & -0.31279400 \\ \mathrm{O} & -1.20133600 & 1.46940900 & -0.74261500 \\ \mathrm{C} & -0.91866400 & 2.55419000 & 0.05755700 \\ \mathrm{H} & -1.26197400 & 3.48295100 & -0.43633100 \\ \mathrm{H} & -1.41958600 & 2.50117700 & 1.04502700 \\ \mathrm{H} & 0.16555900 & 2.67221800 & 0.26090400\end{array}$

\section{$(\mathrm{MeO})(\mathrm{eg}) \mathrm{B}-\mathrm{B}(\mathrm{eg})$ radical anion}

Sum of electronic and zero-point Energies= 622.576305

Sum of electronic and thermal Free Energies= 622.620784

$\begin{array}{llll}\mathrm{C} & -1.67947200 & -1.62771900 & 0.84145100 \\ \mathrm{C} & -1.45908600 & -1.95328300 & -0.64363500 \\ \mathrm{H} & -2.50175100 & -2.19635100 & 1.29220600\end{array}$




$\begin{array}{lrrr}\mathrm{H} & -0.76493100 & -1.80455300 & 1.42822000 \\ \mathrm{H} & -2.34615000 & -2.41131400 & -1.10590000 \\ \mathrm{H} & -0.60089800 & -2.61760500 & -0.80335100 \\ \mathrm{~B} & -1.56404200 & 0.26751200 & -0.34330900 \\ \mathrm{C} & 3.16239600 & 0.62029700 & -0.32280100 \\ \mathrm{C} & 3.04137900 & -0.83525900 & 0.15765400 \\ \mathrm{H} & 3.33488600 & 0.69566600 & -1.40359600 \\ \mathrm{H} & 3.95011600 & 1.17917900 & 0.19456500 \\ \mathrm{H} & 3.33694200 & -1.56490300 & -0.60435900 \\ \mathrm{H} & 3.62074500 & -1.03042500 & 1.06898200 \\ \mathrm{~B} & 1.07464400 & 0.21344200 & 0.36017000 \\ \mathrm{O} & -1.21034800 & -0.69906600 & -1.25798200 \\ \mathrm{O} & -1.98308200 & -0.24163400 & 0.86684900 \\ \mathrm{O} & 1.89172100 & 1.21795200 & -0.02418300 \\ \mathrm{O} & 1.64678800 & -1.01232100 & 0.45582000 \\ \mathrm{O} & -1.53102000 & 1.58253200 & -0.64948000 \\ \mathrm{C} & -1.60515700 & 2.50867700 & 0.41017500 \\ \mathrm{H} & -1.57004700 & 3.51546400 & -0.01751600 \\ \mathrm{H} & -2.53301500 & 2.39195200 & 0.98598700 \\ \mathrm{H} & -0.75174800 & 2.38213500 & 1.09519300\end{array}$

\section{$\mathrm{Cp}^{\mathrm{A} 3} \mathrm{Rh}(\mathrm{III})(\mathrm{OMe})_{2}$}

Sum of electronic and zero-point Energies= 1699.920438

Sum of electronic and thermal Free Energies= 1699.989514

$\begin{array}{lrrr}\mathrm{Rh} & 1.56312700 & -0.68745600 & 1.01107500 \\ \mathrm{C} & 0.67741300 & -1.15949600 & -0.93872500 \\ \mathrm{C} & 0.70392800 & 0.29365900 & -0.72886700 \\ \mathrm{C} & 2.05516300 & 0.74391700 & -0.63982100 \\ \mathrm{C} & 2.85085800 & -0.44446600 & -0.69976000 \\ \mathrm{C} & 2.02173800 & -1.57941600 & -0.95944800 \\ \mathrm{C} & 2.90620500 & -2.76267400 & -1.14912700 \\ \mathrm{H} & 2.60723600 & -3.65563400 & -0.58589300 \\ \mathrm{H} & 2.97175200 & -3.03303000 & -2.21905100 \\ \mathrm{O} & 1.40834000 & -2.08967600 & 2.37672700 \\ \mathrm{O} & 1.68416000 & 0.55429400 & 2.52819500 \\ \mathrm{C} & 1.58942700 & 0.13044900 & 3.84633900 \\ \mathrm{H} & 2.37273400 & -0.59773300 & 4.11701000 \\ \mathrm{H} & 1.69526600 & 1.01626500 & 4.49532000 \\ \mathrm{H} & 0.62392200 & -0.35242400 & 4.07561000 \\ \mathrm{C} & 1.55183900 & -3.41334600 & 1.99695900 \\ \mathrm{H} & 1.37217800 & -4.06712700 & 2.86770200 \\ \mathrm{H} & 0.83879000 & -3.72481600 & 1.20539100 \\ \mathrm{H} & 2.57307500 & -3.63421200 & 1.62802400 \\ \mathrm{C} & 4.26677300 & -0.90703800 & -0.72027700 \\ \mathrm{H} & 4.87336200 & -0.56391200 & 0.12594300 \\ \mathrm{H} & 4.75957500 & -0.58096200 & -1.65557000 \\ \mathrm{O} & 4.16854100 & -2.32483900 & -0.65780900 \\ \mathrm{C} & -0.54796200 & -1.95793000 & -1.14422200 \\ \mathrm{C} & -1.27742100 & -2.46118200 & -0.06280500 \\ \mathrm{C} & -1.00115500 & -2.18764800 & -2.44727200 \\ \mathrm{C} & -2.43626800 & -3.19856700 & -0.28588100 \\ \mathrm{H} & -0.92173700 & -2.26852500 & 0.95138200 \\ \mathrm{C} & -2.15951000 & -2.92653800 & -2.66568100 \\ \mathrm{H} & -0.44180900 & -1.77263200 & -3.28601700 \\ \mathrm{C} & -2.87680000 & -3.43543400 & -1.58496900 \\ \mathrm{H} & -2.99639600 & -3.58971900 & 0.56188200 \\ \mathrm{H} & -2.50262400 & -3.10601700 & -3.68327900 \\ \mathrm{H} & -3.78114400 & -4.01718800 & -1.75713800 \\ \mathrm{C} & 2.52406000 & 2.12550500 & -0.46052100 \\ \mathrm{C} & 2.13690300 & 3.10753100 & -1.37874400 \\ \mathrm{C} & 3.37952100 & 2.45930300 & 0.59457500 \\ \mathrm{C} & 2.61156800 & 4.40788800 & -1.24494800 \\ \mathrm{H} & 1.47500800 & 2.83193000 & -2.20154900 \\ \mathrm{C} & 3.85041200 & 3.76043300 & 0.71908700\end{array}$

$\begin{array}{lrrr}\mathrm{H} & 3.61963200 & 1.70480600 & 1.34318200 \\ \mathrm{C} & 3.47149800 & 4.73481200 & -0.20071900 \\ \mathrm{H} & 2.31204400 & 5.16803100 & -1.96488200 \\ \mathrm{H} & 4.50801700 & 4.01842900 & 1.54763100 \\ \mathrm{H} & 3.84296600 & 5.75349800 & -0.09899400 \\ \mathrm{C} & -0.52911800 & 1.12102100 & -0.64654500 \\ \mathrm{H} & -1.28152500 & 0.62201000 & -0.02147600 \\ \mathrm{H} & -0.30529700 & 2.08300200 & -0.16017100 \\ \mathrm{C} & -1.06777900 & 1.39931100 & -2.04161700 \\ \mathrm{O} & -0.33252400 & 1.49261000 & -3.01284100 \\ \mathrm{~N} & -2.41947800 & 1.57064800 & -2.14726800 \\ \mathrm{H} & -2.72603500 & 1.74246900 & -3.10015200 \\ \mathrm{C} & -3.39231300 & 1.32973000 & -1.13209400 \\ \mathrm{C} & -3.90502900 & 0.04270800 & -0.94435600 \\ \mathrm{C} & -3.82196900 & 2.36294100 & -0.30828900 \\ \mathrm{C} & -4.83275300 & -0.19957300 & 0.05145600 \\ \mathrm{H} & -3.55197800 & -0.77087200 & -1.57962100 \\ \mathrm{C} & -4.75781600 & 2.13108900 & 0.69653000 \\ \mathrm{H} & -3.41172000 & 3.36142900 & -0.45436900 \\ \mathrm{C} & -5.26701600 & 0.84369900 & 0.87803600 \\ \mathrm{H} & -5.23542300 & -1.19680500 & 0.21931300 \\ \mathrm{H} & -5.07484000 & 2.95512800 & 1.33024900 \\ \mathrm{O} & -6.17491800 & 0.50518000 & 1.82105300 \\ \mathrm{C} & -6.62270900 & 1.51037700 & 2.69740000 \\ \mathrm{H} & -7.13284200 & 2.32127900 & 2.15586500 \\ \mathrm{H} & -7.33239200 & 1.03235200 & 3.37715900 \\ \mathrm{H} & -5.79308000 & 1.93502400 & 3.28222700\end{array}$

\section{$\mathrm{Cp}^{\mathrm{A} 3} \mathrm{Rh}(\mathrm{II})(\mathrm{OMe})_{2}$ anion}

Sum of electronic and zero-point Energies= 1699.979865

Sum of electronic and thermal Free Energies= 1700.050429

\begin{tabular}{crrr}
$\mathrm{Rh}$ & 1.49121600 & -1.05119800 & 0.74356300 \\
$\mathrm{C}$ & 0.31905400 & -0.77377600 & -1.16271800 \\
$\mathrm{C}$ & 0.77164100 & 0.50910000 & -0.65042500 \\
$\mathrm{C}$ & 2.20926800 & 0.54660000 & -0.67433500 \\
$\mathrm{C}$ & 2.62079700 & -0.73638000 & -1.16171800 \\
$\mathrm{C}$ & 1.48908700 & -1.48294700 & -1.54609000 \\
$\mathrm{C}$ & 1.93613200 & -2.77565600 & -2.12906300 \\
$\mathrm{H}$ & 1.54686400 & -3.65492500 & -1.59098900 \\
$\mathrm{H}$ & 1.64432400 & -2.88307700 & -3.19163300 \\
$\mathrm{O}$ & 0.05118500 & -1.27031300 & 2.22296100 \\
$\mathrm{O}$ & 3.03456400 & -1.56046100 & 1.96232700 \\
$\mathrm{C}$ & 2.95507100 & -1.20458200 & 3.29121000 \\
$\mathrm{H}$ & 1.92002200 & -1.21301200 & 3.68095600 \\
$\mathrm{H}$ & 3.55586200 & -1.89400900 & 3.92229900 \\
$\mathrm{H}$ & 3.35541100 & -0.17993800 & 3.48266000 \\
$\mathrm{C}$ & -0.14507000 & -2.58385200 & 2.57310300 \\
$\mathrm{H}$ & -0.86060000 & -2.66055100 & 3.42194800 \\
$\mathrm{H}$ & -0.57576400 & -3.20715800 & 1.75048800 \\
$\mathrm{H}$ & 0.78252600 & -3.10320900 & 2.90154800 \\
$\mathrm{C}$ & 3.84497500 & -1.52252500 & -1.47722500 \\
$\mathrm{H}$ & 4.44117700 & -1.74690100 & -0.57893400 \\
$\mathrm{H}$ & 4.50050600 & -1.02442400 & -2.21388100 \\
$\mathrm{O}$ & 3.35701800 & -2.74775300 & -2.03246200 \\
$\mathrm{C}$ & -1.07711400 & -1.21284600 & -1.33923000 \\
$\mathrm{C}$ & -1.92561000 & -1.37232300 & -0.23340400 \\
$\mathrm{C}$ & -1.56251000 & -1.50993900 & -2.61729900 \\
$\mathrm{C}$ & -3.22900200 & -1.82000500 & -0.41899900 \\
$\mathrm{H}$ & -1.51208400 & -1.19096000 & 0.76794500 \\
$\mathrm{C}$ & -2.86695900 & -1.96223200 & -2.79518200 \\
$\mathrm{H}$ & -0.89950000 & -1.38341300 & -3.47487200 \\
$\mathrm{C}$ & -3.70437200 & -2.11808700 & -1.69462800 \\
$\mathrm{H}$ & -3.88122100 & -1.94725800 & 0.44625900 \\
$\mathrm{H}$ & -3.22806800 & -2.19709000 & -3.79696300 \\
& & & \\
\hline
\end{tabular}




\begin{tabular}{lrrr}
$\mathrm{H}$ & -4.72751000 & -2.47110900 & -1.82940200 \\
$\mathrm{C}$ & 3.08876600 & 1.61802200 & -0.19274000 \\
$\mathrm{C}$ & 2.92722500 & 2.93865500 & -0.62984600 \\
$\mathrm{C}$ & 4.11876000 & 1.31720900 & 0.71312800 \\
$\mathrm{C}$ & 3.78422900 & 3.93708800 & -0.17521100 \\
$\mathrm{H}$ & 2.12707600 & 3.16469500 & -1.33667900 \\
$\mathrm{C}$ & 4.97498100 & 2.31915900 & 1.15130000 \\
$\mathrm{H}$ & 4.18303400 & 0.29798100 & 1.10873100 \\
$\mathrm{C}$ & 4.81458400 & 3.63199000 & 0.70887700 \\
$\mathrm{H}$ & 3.64820900 & 4.96174400 & -0.52271200 \\
$\mathrm{H}$ & 5.76390100 & 2.07657000 & 1.86354300 \\
$\mathrm{H}$ & 5.48611100 & 4.41561100 & 1.06077200 \\
$\mathrm{C}$ & -0.14896000 & 1.58237600 & -0.16670900 \\
$\mathrm{H}$ & -0.92853700 & 1.12632900 & 0.45273900 \\
$\mathrm{H}$ & 0.41124200 & 2.27754900 & 0.47804900 \\
$\mathrm{C}$ & -0.72643400 & 2.38817500 & -1.31158600 \\
$\mathrm{O}$ & -0.03150600 & 2.89165800 & -2.18098500 \\
$\mathrm{~N}$ & -2.09706900 & 2.56243800 & -1.37981500 \\
$\mathrm{H}$ & -2.35876600 & 2.99965300 & -2.25786600 \\
$\mathrm{C}$ & -3.14462600 & 2.01116200 & -0.61042800 \\
$\mathrm{C}$ & -4.21896900 & 1.40063500 & -1.26443400 \\
$\mathrm{C}$ & -3.16703600 & 2.09372500 & 0.77922500 \\
$\mathrm{C}$ & -5.26917400 & 0.86035800 & -0.54548400 \\
$\mathrm{H}$ & -4.20048100 & 1.31305100 & -2.35074100 \\
$\mathrm{C}$ & -4.19966500 & 1.51527000 & 1.51279400 \\
$\mathrm{H}$ & -2.36234800 & 2.60808800 & 1.30129100 \\
$\mathrm{C}$ & -5.25468900 & 0.89164200 & 0.85029800 \\
$\mathrm{H}$ & -6.09519200 & 0.35664400 & -1.04449300 \\
$\mathrm{H}$ & -4.16514200 & 1.56596100 & 2.59799600 \\
$\mathrm{O}$ & -6.30401300 & 0.28383800 & 1.47044500 \\
$\mathrm{C}$ & -6.23829200 & 0.15569600 & 2.86583300 \\
$\mathrm{H}$ & -6.26890300 & 1.13460700 & 3.37052400 \\
$\mathrm{H}$ & -7.11527200 & -0.42485700 & 3.16694300 \\
$\mathrm{H}$ & -5.32663000 & -0.37578400 & 3.18048900 \\
& & & \\
\hline & & & \\
& & &
\end{tabular}

\section{$\mathrm{Cp}^{\mathrm{A} 3} \mathrm{Rh}(\mathrm{II})(\mathrm{OMe})$}

Sum of electronic and zero-point Energies= 1584.898741

Sum of electronic and thermal Free Energies= 1584.964736

$\begin{array}{crrr}\mathrm{Rh} & 1.48302000 & -0.98222200 & 1.23280700 \\ \mathrm{C} & 0.52273200 & -1.09740600 & -0.77182900 \\ \mathrm{C} & 0.93244000 & 0.26535400 & -0.43760800 \\ \mathrm{C} & 2.37928600 & 0.35192500 & -0.44283300 \\ \mathrm{C} & 2.82269900 & -0.99288100 & -0.57272100 \\ \mathrm{C} & 1.71926500 & -1.84642800 & -0.83957000 \\ \mathrm{C} & 2.23968400 & -3.21181800 & -1.13054600 \\ \mathrm{H} & 1.74331400 & -4.02264100 & -0.58282700 \\ \mathrm{H} & 2.16857800 & -3.43766000 & -2.21175300 \\ \mathrm{O} & 0.40645100 & -0.58418400 & 2.86603900 \\ \mathrm{C} & 1.18783100 & -1.46107700 & 3.55056700 \\ \mathrm{H} & 1.77833400 & -1.00718900 & 4.36664200 \\ \mathrm{H} & 0.65803000 & -2.35723200 & 3.92098400 \\ \mathrm{H} & 2.02632400 & -1.92409200 & 2.86039600 \\ \mathrm{C} & 4.06570600 & -1.80868500 & -0.71833800 \\ \mathrm{H} & 4.79425600 & -1.70465900 & 0.09494700 \\ \mathrm{H} & 4.57955500 & -1.56837400 & -1.66807000 \\ \mathrm{O} & 3.59994600 & -3.15419900 & -0.71671800 \\ \mathrm{C} & -0.85061000 & -1.59591200 & -0.95744100 \\ \mathrm{C} & -1.79650700 & -1.54185500 & 0.07504000 \\ \mathrm{C} & -1.21286900 & -2.16289000 & -2.18360300 \\ \mathrm{C} & -3.07470600 & -2.04960400 & -0.12191800 \\ \mathrm{H} & -1.50522500 & -1.13594600 & 1.04611200 \\ \mathrm{C} & -2.49360400 & -2.67143600 & -2.37543200 \\ \mathrm{H} & -0.47882000 & -2.19909200 & -2.98943800 \\ \mathrm{C} & -3.42623300 & -2.61680200 & -1.34428500\end{array}$

$\begin{array}{rcc}-3.80192600 & -2.00526300 & 0.68773100 \\ -2.76093200 & -3.11576800 & -3.33312900 \\ -4.42885100 & -3.01635200 & -1.49175000 \\ 3.21911800 & 1.53326800 & -0.22662300 \\ 2.94470500 & 2.74264200 & -0.87771900 \\ 4.34315600 & 1.45338300 & 0.60733200 \\ 3.77037200 & 3.84491800 & -0.68109600 \\ 2.10266500 & 2.79300500 & -1.56883400 \\ 5.16624900 & 2.55442200 & 0.79495300 \\ 4.54711500 & 0.52040900 & 1.13471700 \\ 4.87986100 & 3.75768800 & 0.15316500 \\ 3.54863900 & 4.77819200 & -1.19677700 \\ 6.03061000 & 2.47735900 & 1.45281800 \\ 5.52314300 & 4.62340600 & 0.30251700 \\ -0.04696100 & 1.38425900 & -0.28637100 \\ -0.87552800 & 1.06114200 & 0.35307300 \\ 0.43552900 & 2.23603600 & 0.21576000 \\ -0.53052300 & 1.85082700 & -1.64868900 \\ 0.25129300 & 2.07378000 & -2.56106300 \\ -1.87744900 & 2.02081200 & -1.84584200 \\ -2.07982900 & 2.24574800 & -2.81566200 \\ -2.98071600 & 1.69821000 & -1.01771500 \\ -3.99974900 & 0.89144000 & -1.53244600 \\ -3.09971800 & 2.19125300 & 0.27768400 \\ -5.10265400 & 0.57535200 & -0.76205900 \\ -3.90214500 & 0.48651400 & -2.53982300 \\ -4.18677100 & 1.84293700 & 1.07612500 \\ -2.33667600 & 2.86107800 & 0.67165900 \\ -5.19679100 & 1.03406800 & 0.55578500 \\ -5.89843800 & -0.05972300 & -1.14701300 \\ -4.24045200 & 2.22781900 & 2.09110000 \\ -6.29775000 & 0.63940100 & 1.24058400 \\ -6.43430300 & 1.07658900 & 2.56966400 \\ -6.49024200 & 2.17408200 & 2.63161900 \\ -7.36970500 & 0.64985600 & 2.94055800 \\ -5.60293200 & 0.72485300 & 3.19944900\end{array}$

\section{$\mathrm{Cp}^{\mathrm{A} 3} \mathrm{Rh}(\mathrm{I})(\mathrm{OMe})$ anion}

Sum of electronic and zero-point Energies= 1584.938960

Sum of electronic and thermal Free Energies= 1585.004216

$\begin{array}{crrr}\mathrm{Rh} & 0.95680000 & -0.68416700 & 1.54750500 \\ \mathrm{C} & 0.79111300 & -1.37082900 & -0.46516500 \\ \mathrm{C} & 1.09547600 & 0.09057900 & -0.46644300 \\ \mathrm{C} & 2.49895100 & 0.30682000 & -0.23339900 \\ \mathrm{C} & 2.91406100 & -0.94261600 & 0.33087300 \\ \mathrm{C} & 1.98016900 & -1.96742100 & 0.04187000 \\ \mathrm{C} & 2.63686800 & -3.28622200 & 0.26852400 \\ \mathrm{H} & 2.05583500 & -3.99744300 & 0.87283500 \\ \mathrm{H} & 2.88990100 & -3.78940600 & -0.68886200 \\ \mathrm{O} & -0.64147400 & 0.31487400 & 2.28803700 \\ \mathrm{C} & -1.73271000 & -0.42043500 & 2.66875400 \\ \mathrm{H} & -2.52090600 & 0.24379100 & 3.10018300 \\ \mathrm{H} & -2.22479400 & -0.96011100 & 1.82546000 \\ \mathrm{H} & -1.52556900 & -1.18774300 & 3.44839400 \\ \mathrm{C} & 4.17070900 & -1.59828100 & 0.79552300 \\ \mathrm{H} & 4.55643200 & -1.22523100 & 1.75596200 \\ \mathrm{H} & 4.97918300 & -1.48422100 & 0.04508700 \\ \mathrm{O} & 3.83452400 & -2.97165700 & 0.97283300 \\ \mathrm{C} & -0.36536700 & -2.08526900 & -1.00334400 \\ \mathrm{C} & -1.68228600 & -1.60526900 & -0.90650300 \\ \mathrm{C} & -0.17354700 & -3.31821700 & -1.64965600 \\ \mathrm{C} & -2.74808300 & -2.32473400 & -1.42928200 \\ \mathrm{H} & -1.87907300 & -0.67939000 & -0.36673900 \\ \mathrm{C} & -1.24182600 & -4.04034800 & -2.16531500\end{array}$




$\begin{array}{lrrr}\mathrm{H} & 0.84149500 & -3.70093900 & -1.76015800 \\ \mathrm{C} & -2.54046700 & -3.54909400 & -2.06218500 \\ \mathrm{H} & -3.75734400 & -1.92588200 & -1.31878200 \\ \mathrm{H} & -1.05537500 & -4.99449500 & -2.65951200 \\ \mathrm{H} & -3.38064500 & -4.11457500 & -2.46443200 \\ \mathrm{C} & 3.26118200 & 1.52359900 & -0.33254400 \\ \mathrm{C} & 2.98107900 & 2.50418900 & -1.31101700 \\ \mathrm{C} & 4.36121200 & 1.76558700 & 0.51978100 \\ \mathrm{C} & 3.73635200 & 3.66541800 & -1.39803800 \\ \mathrm{H} & 2.19499100 & 2.30835800 & -2.04146400 \\ \mathrm{C} & 5.11908600 & 2.92067600 & 0.41512500 \\ \mathrm{H} & 4.58729000 & 1.03906700 & 1.30155000 \\ \mathrm{C} & 4.81168000 & 3.89154100 & -0.53927100 \\ \mathrm{H} & 3.49396700 & 4.39861400 & -2.16895000 \\ \mathrm{H} & 5.95538400 & 3.07476600 & 1.09886400 \\ \mathrm{H} & 5.40479100 & 4.80232400 & -0.61732300 \\ \mathrm{C} & 0.08502000 & 1.17316900 & -0.70187300 \\ \mathrm{H} & -0.72307900 & 1.03366100 & 0.02425900 \\ \mathrm{H} & 0.54623300 & 2.13761000 & -0.42980100 \\ \mathrm{C} & -0.42722600 & 1.32486800 & -2.10959900 \\ \mathrm{O} & 0.26648100 & 1.18564200 & -3.10289600 \\ \mathrm{~N} & -1.75655300 & 1.71132900 & -2.26867300 \\ \mathrm{H} & -2.01534000 & 1.67551300 & -3.24997800 \\ \mathrm{C} & -2.81975000 & 1.64545900 & -1.34086800 \\ \mathrm{C} & -3.98516000 & 0.95055900 & -1.68336900 \\ \mathrm{C} & -2.74665300 & 2.23846700 & -0.08196300 \\ \mathrm{C} & -5.01737000 & 0.80201300 & -0.77250200 \\ \mathrm{H} & -4.05318700 & 0.47869900 & -2.66440200 \\ \mathrm{C} & -3.76137100 & 2.05952100 & 0.85334200 \\ \mathrm{H} & -1.87259700 & 2.82333600 & 0.19381800 \\ \mathrm{C} & -4.89762400 & 1.32786600 & 0.51616700 \\ \mathrm{H} & -5.91699300 & 0.24234300 & -1.02333700 \\ \mathrm{H} & -3.63714900 & 2.48877100 & 1.84409400 \\ \mathrm{O} & -5.93645700 & 1.08414000 & 1.36156400 \\ \mathrm{C} & -5.75601000 & 1.43029500 & 2.71026600 \\ \mathrm{H} & -5.68944600 & 2.52150000 & 2.84811500 \\ \mathrm{H} & -6.63449900 & 1.05918100 & 3.24664300 \\ \mathrm{H} & -4.84928700 & 0.96477500 & 3.12783300\end{array}$

\begin{tabular}{|c|c|c|c|}
\hline $\mathrm{H}$ & 1.57392000 & -0.69735200 & -4.25851800 \\
\hline $\mathrm{H}$ & 2.84660500 & -1.31634100 & -3.18225800 \\
\hline $\mathrm{C}$ & -1.41765000 & -0.71090100 & 1.13925100 \\
\hline $\mathrm{N}$ & -2.65147300 & -1.19690000 & 1.53123700 \\
\hline $\mathrm{H}$ & -2.55478000 & -2.07391200 & 2.03358900 \\
\hline $\mathrm{C}$ & -3.95121600 & -0.82718400 & 1.11164100 \\
\hline $\mathrm{C}$ & -4.77835000 & -1.76965800 & 0.50645400 \\
\hline $\mathrm{C}$ & -4.45122400 & 0.46031900 & 1.33770500 \\
\hline $\mathrm{C}$ & -6.07824100 & -1.44785500 & 0.12407000 \\
\hline $\mathrm{H}$ & -4.39079800 & -2.77325600 & 0.32943200 \\
\hline $\mathrm{C}$ & -5.73061200 & 0.79891200 & 0.93490800 \\
\hline $\mathrm{H}$ & -3.82425300 & 1.19362200 & 1.84365900 \\
\hline $\mathrm{C}$ & -6.55628100 & -0.15346700 & 0.32869200 \\
\hline $\mathrm{H}$ & -6.69964700 & -2.20918900 & -0.34076600 \\
\hline $\mathrm{H}$ & -6.13010100 & 1.79771500 & 1.09833700 \\
\hline $\mathrm{O}$ & 3.00647900 & 0.68285800 & -3.60318600 \\
\hline $\mathrm{C}$ & -0.30800800 & -1.93237700 & -1.52014400 \\
\hline $\mathrm{C}$ & -1.67397500 & -2.23901400 & -1.57520800 \\
\hline $\mathrm{C}$ & 0.60950800 & -2.99254000 & -1.51677700 \\
\hline $\mathrm{C}$ & -2.10677200 & -3.56004600 & -1.61094900 \\
\hline $\mathrm{H}$ & -2.40500900 & -1.43055900 & -1.62210300 \\
\hline $\mathrm{C}$ & 0.17607200 & -4.31011900 & -1.55705500 \\
\hline $\mathrm{H}$ & 1.67421800 & -2.76600100 & -1.44382900 \\
\hline $\mathrm{C}$ & -1.18574000 & -4.60264100 & -1.60252900 \\
\hline $\mathrm{H}$ & -3.17430900 & -3.77394500 & -1.67083600 \\
\hline $\mathrm{H}$ & 0.90661300 & -5.11796900 & -1.54336200 \\
\hline $\mathrm{H}$ & -1.52459800 & -5.63685700 & -1.63598100 \\
\hline $\mathrm{C}$ & 0.13783300 & 3.13744300 & -0.73263900 \\
\hline $\mathrm{C}$ & 1.18392100 & 4.02617000 & -0.44976300 \\
\hline $\mathrm{C}$ & -1.17559700 & 3.61581900 & -0.65602200 \\
\hline $\mathrm{C}$ & 0.92534600 & 5.34437700 & -0.09844500 \\
\hline $\mathrm{H}$ & 2.20901100 & 3.65452200 & -0.49109200 \\
\hline $\mathrm{C}$ & -1.43563500 & 4.93442500 & -0.29566400 \\
\hline $\mathrm{H}$ & -2.00257900 & 2.95149100 & -0.90656100 \\
\hline $\mathrm{C}$ & -0.38740500 & 5.80376100 & -0.01475400 \\
\hline $\mathrm{H}$ & 1.75373900 & 6.01676300 & 0.12044700 \\
\hline $\mathrm{H}$ & -2.46543200 & 5.28558600 & -0.24413800 \\
\hline $\mathrm{H}$ & -0.59060500 & 6.83563600 & 0.26713600 \\
\hline $\mathrm{O}$ & -7.79572100 & 0.27365400 & -0.01503800 \\
\hline $\mathrm{C}$ & -8.66145300 & -0.64995700 & -0.62456500 \\
\hline $\mathrm{H}$ & -9.59488400 & -0.11606900 & -0.82033500 \\
\hline $\mathrm{H}$ & -8.25303300 & -1.02149000 & -1.57686700 \\
\hline $\mathrm{H}$ & -8.86883300 & -1.50736600 & 0.03403600 \\
\hline $\mathrm{C}$ & 3.77685200 & -1.10835000 & 1.55654000 \\
\hline $\mathrm{C}$ & 4.80988600 & -1.96975900 & 1.93617700 \\
\hline $\mathrm{C}$ & 5.68199400 & -2.46311900 & 0.98196600 \\
\hline $\mathrm{H}$ & 4.92672500 & -2.23263200 & 2.98558700 \\
\hline $\mathrm{C}$ & 4.48771200 & -1.20717000 & -0.66415900 \\
\hline $\mathrm{C}$ & 5.52205500 & -2.06794800 & -0.34744200 \\
\hline $\mathrm{H}$ & 6.48976800 & -3.13369900 & 1.26824600 \\
\hline $\mathrm{H}$ & 4.33665600 & -0.83754000 & -1.67855200 \\
\hline $\mathrm{H}$ & 6.19762200 & -2.41043800 & -1.12728100 \\
\hline $\mathrm{N}$ & 3.61921000 & -0.74602800 & 0.25335500 \\
\hline $\mathrm{O}$ & -0.40533600 & -1.24530600 & 1.54564000 \\
\hline $\mathrm{C}$ & 2.11749900 & 0.62787900 & 2.00629600 \\
\hline $\mathrm{H}$ & 2.65468700 & 1.34124800 & 1.25959100 \\
\hline
\end{tabular}

\section{INT1}

Sum of electronic and zero-point Energies = $-1948.706363$

Sum of electronic and thermal Free Energies = $-1948.780432$

$\begin{array}{crrr}\text { Rh } & 1.96973100 & 0.44701500 & -0.18812000 \\ \mathrm{C} & 2.81991700 & -0.50631100 & 2.47525800 \\ \mathrm{C} & 1.16611600 & 1.24104400 & 2.83207200 \\ \mathrm{C} & -0.34509800 & 0.54802200 & -0.79777000 \\ \mathrm{C} & 0.43096100 & 1.75442100 & -1.14704500 \\ \mathrm{C} & 1.36950500 & 1.36316300 & -2.14057200 \\ \mathrm{C} & 1.31849000 & -0.04462300 & -2.22643900 \\ \mathrm{C} & 0.15150300 & -0.54680300 & -1.51904800 \\ \mathrm{C} & -1.42204400 & 0.51888500 & 0.24198200 \\ \mathrm{C} & 2.51721600 & -1.02894200 & 3.73374700 \\ \mathrm{C} & 1.54619100 & -0.43099400 & 4.52509300 \\ \mathrm{C} & 0.87071400 & 0.70670300 & 4.07579400 \\ \mathrm{C} & 2.32361700 & 1.86298900 & -3.17427900 \\ \mathrm{H} & 0.66553800 & 2.14412500 & 2.47889300 \\ \mathrm{H} & 3.01292400 & -1.93502100 & 4.08253000 \\ \mathrm{H} & 0.11439100 & 1.17208100 & 4.70635500 \\ \mathrm{H} & 1.31014000 & -0.85397700 & 5.50004000 \\ \mathrm{H} & -1.29316400 & 1.38924300 & 0.90674400 \\ \mathrm{H} & 1.78973400 & 2.32303800 & -4.02848100 \\ \mathrm{H} & -2.41302500 & 0.64767800 & -0.21822600 \\ \mathrm{H} & 3.07760600 & 2.57765800 & -2.82248500 \\ \mathrm{C} & 2.18734400 & -0.45648500 & -3.36788200\end{array}$

\section{TS1}

Sum of electronic and zero-point Energies = $-1948.704942$

Sum of electronic and thermal Free Energies = $-1948.778046$

$\begin{array}{cccc}\mathrm{Rh} & 1.97050400 & 0.36696300 & -0.26483300 \\ \mathrm{C} & 2.68028400 & 0.09237400 & 2.55903100 \\ \mathrm{C} & 1.24414400 & 2.01528700 & 2.24651100\end{array}$




\begin{tabular}{|c|c|c|c|}
\hline $\mathrm{C}$ & -0.27045900 & 0.38946700 & -0.93106000 \\
\hline $\mathrm{C}$ & 0.49315100 & 1.55578300 & -1.38788800 \\
\hline $\mathrm{C}$ & 1.42709700 & 1.07292500 & -2.36390500 \\
\hline $\mathrm{C}$ & 1.36310100 & -0.31939100 & -2.37398900 \\
\hline $\mathrm{C}$ & 0.26285100 & -0.76548900 & -1.54423100 \\
\hline $\mathrm{C}$ & -1.34096600 & 0.43499600 & 0.11456000 \\
\hline $\mathrm{C}$ & 2.54943900 & 0.22881900 & 3.94409900 \\
\hline $\mathrm{C}$ & 1.76229900 & 1.23697100 & 4.47793800 \\
\hline $\mathrm{C}$ & 1.11253400 & 2.13115700 & 3.62395100 \\
\hline $\mathrm{C}$ & 2.36986100 & 1.51732400 & -3.43130700 \\
\hline $\mathrm{H}$ & 0.75253800 & 2.73730500 & 1.59047900 \\
\hline $\mathrm{H}$ & 3.04829500 & -0.47198400 & 4.61425200 \\
\hline $\mathrm{H}$ & 0.50003500 & 2.92986200 & 4.04204500 \\
\hline $\mathrm{H}$ & 1.65842600 & 1.33426100 & 5.55707900 \\
\hline $\mathrm{H}$ & -1.23162300 & 1.37012100 & 0.68868100 \\
\hline $\mathrm{H}$ & 1.82790500 & 1.97345800 & -4.28202000 \\
\hline $\mathrm{H}$ & -2.33823700 & 0.49006600 & -0.34702600 \\
\hline $\mathrm{H}$ & 3.14563400 & 2.22408000 & -3.11038600 \\
\hline $\mathrm{C}$ & 2.23428500 & -0.81892600 & -3.47752000 \\
\hline $\mathrm{H}$ & 1.62837700 & -1.17641100 & -4.33322500 \\
\hline $\mathrm{H}$ & 2.92371500 & -1.63384300 & -3.20957100 \\
\hline $\mathrm{C}$ & -1.30683100 & -0.69713100 & 1.13372900 \\
\hline $\mathrm{N}$ & -2.52941400 & -1.12767700 & 1.61149200 \\
\hline $\mathrm{H}$ & -2.42550900 & -1.94054600 & 2.21075800 \\
\hline $\mathrm{C}$ & -3.83993200 & -0.76399600 & 1.22055700 \\
\hline $\mathrm{C}$ & -4.71681500 & -1.73663900 & 0.74767400 \\
\hline $\mathrm{C}$ & -4.29846400 & 0.55272900 & 1.34399200 \\
\hline $\mathrm{C}$ & -6.02582000 & -1.41698800 & 0.39583500 \\
\hline $\mathrm{H}$ & -4.36371200 & -2.76380700 & 0.65325000 \\
\hline $\mathrm{C}$ & -5.58733200 & 0.88652200 & 0.96904500 \\
\hline $\mathrm{H}$ & -3.63110000 & 1.31314700 & 1.74778100 \\
\hline $\mathrm{C}$ & -6.46315000 & -0.09614600 & 0.49592800 \\
\hline $\mathrm{H}$ & -6.68654800 & -2.20172400 & 0.03605800 \\
\hline $\mathrm{H}$ & -5.95535200 & 1.90697100 & 1.05354400 \\
\hline $\mathrm{O}$ & 3.01349700 & 0.31283300 & -3.84104000 \\
\hline $\mathrm{C}$ & -0.19581600 & -2.15051300 & -1.41765800 \\
\hline $\mathrm{C}$ & -1.56292700 & -2.45332100 & -1.40546300 \\
\hline $\mathrm{C}$ & 0.71809200 & -3.20974100 & -1.36073900 \\
\hline $\mathrm{C}$ & -2.00033800 & -3.77021200 & -1.31995900 \\
\hline $\mathrm{H}$ & -2.29286900 & -1.64725900 & -1.49000200 \\
\hline $\mathrm{C}$ & 0.28119600 & -4.52469600 & -1.27714900 \\
\hline $\mathrm{H}$ & 1.78565800 & -2.98789900 & -1.35148100 \\
\hline $\mathrm{C}$ & -1.08137900 & -4.81251200 & -1.25419900 \\
\hline $\mathrm{H}$ & -3.06944800 & -3.98252300 & -1.32557100 \\
\hline $\mathrm{H}$ & 1.01004200 & -5.33221400 & -1.22324900 \\
\hline $\mathrm{H}$ & -1.42336900 & -5.84428600 & -1.19145000 \\
\hline $\mathrm{C}$ & 0.18648700 & 2.97381900 & -1.12882400 \\
\hline $\mathrm{C}$ & 1.22166400 & 3.90523400 & -0.97154000 \\
\hline $\mathrm{C}$ & -1.13345900 & 3.43538800 & -1.07109500 \\
\hline $\mathrm{C}$ & 0.94590700 & 5.24850200 & -0.75499800 \\
\hline $\mathrm{H}$ & 2.25337700 & 3.55155300 & -0.99121400 \\
\hline $\mathrm{C}$ & -1.41039400 & 4.78035600 & -0.84530100 \\
\hline $\mathrm{H}$ & -1.95354600 & 2.73496300 & -1.22737600 \\
\hline $\mathrm{C}$ & -0.37303700 & 5.69189900 & -0.68507100 \\
\hline $\mathrm{H}$ & 1.76641400 & 5.95330500 & -0.62894200 \\
\hline $\mathrm{H}$ & -2.44534800 & 5.11708100 & -0.80389500 \\
\hline $\mathrm{H}$ & -0.58934300 & 6.74406900 & -0.50753400 \\
\hline $\mathrm{O}$ & -7.70707200 & 0.33061800 & 0.16922000 \\
\hline $\mathrm{C}$ & -8.62303700 & -0.62213500 & -0.30782100 \\
\hline $\mathrm{H}$ & -9.55189000 & -0.08311300 & -0.51105000 \\
\hline $\mathrm{H}$ & -8.27019400 & -1.09399100 & -1.23763300 \\
\hline $\mathrm{H}$ & -8.81851500 & -1.40658400 & 0.43930500 \\
\hline $\mathrm{C}$ & 3.45797000 & -0.95162100 & 1.91537200 \\
\hline $\mathrm{C}$ & 4.29777600 & -1.86078500 & 2.56822600 \\
\hline $\mathrm{C}$ & 5.00711300 & -2.79484100 & 1.83650100 \\
\hline $\mathrm{H}$ & 4.38885900 & -1.82381400 & 3.65140300 \\
\hline
\end{tabular}

$\begin{array}{lrrr}\mathrm{C} & 4.04844900 & -1.87663000 & -0.14392000 \\ \mathrm{C} & 4.88685000 & -2.80404100 & 0.44640500 \\ \mathrm{H} & 5.65889500 & -3.50610200 & 2.34034600 \\ \mathrm{H} & 3.93515100 & -1.81767800 & -1.22665200 \\ \mathrm{H} & 5.44010500 & -3.50756700 & -0.17023600 \\ \mathrm{~N} & 3.34009000 & -0.98349000 & 0.56180600 \\ \mathrm{O} & -0.28540500 & -1.20215200 & 1.55583200 \\ \mathrm{C} & 2.00505700 & 0.97928600 & 1.68620800 \\ \mathrm{H} & 2.85490400 & 1.47702800 & 0.54428000\end{array}$

\section{INT2}

Sum of electronic and zero-point Energies = $-1948.713116$

Sum of electronic and thermal Free Energies $=$ $-1948.786808$

\begin{tabular}{|c|c|c|c|}
\hline $\mathrm{Rh}$ & 2.04689300 & 0.38031400 & -0.25272200 \\
\hline $\mathrm{C}$ & 2.51499100 & 0.05303000 & 2.60291000 \\
\hline $\mathrm{C}$ & 1.21177400 & 2.03317900 & 2.18173600 \\
\hline $\mathrm{C}$ & -0.21534500 & 0.40352800 & -0.93291400 \\
\hline $\mathrm{C}$ & 0.54802300 & 1.57902700 & -1.34939300 \\
\hline $\mathrm{C}$ & 1.50166600 & 1.10327000 & -2.31294000 \\
\hline $\mathrm{C}$ & 1.40165900 & -0.28515700 & -2.40915600 \\
\hline $\mathrm{C}$ & 0.31047000 & -0.73760500 & -1.58651600 \\
\hline $\mathrm{C}$ & -1.29533200 & 0.41601700 & 0.10329500 \\
\hline $\mathrm{C}$ & 2.39708800 & 0.26426000 & 3.98044800 \\
\hline $\mathrm{C}$ & 1.68107500 & 1.35052600 & 4.45920800 \\
\hline $\mathrm{C}$ & 1.09420000 & 2.23556900 & 3.55504000 \\
\hline $\mathrm{C}$ & 2.46088400 & 1.58187600 & -3.35225300 \\
\hline $\mathrm{H}$ & 0.75862100 & 2.75350800 & 1.49731400 \\
\hline $\mathrm{H}$ & 2.86244300 & -0.42439300 & 4.68697900 \\
\hline $\mathrm{H}$ & 0.54121100 & 3.09913000 & 3.92565900 \\
\hline $\mathrm{H}$ & 1.58663800 & 1.51452900 & 5.53129200 \\
\hline $\mathrm{H}$ & -1.19067000 & 1.33056600 & 0.71050100 \\
\hline $\mathrm{H}$ & 1.92505700 & 2.08634800 & -4.17913500 \\
\hline $\mathrm{H}$ & -2.28967200 & 0.48434200 & -0.36282700 \\
\hline $\mathrm{H}$ & 3.24347500 & 2.26061000 & -2.99251300 \\
\hline $\mathrm{C}$ & 2.27806200 & -0.74418500 & -3.52688300 \\
\hline $\mathrm{H}$ & 1.68028400 & -1.03704700 & -4.41216400 \\
\hline $\mathrm{H}$ & 2.94623700 & -1.58632800 & -3.29469400 \\
\hline $\mathrm{C}$ & -1.26390500 & -0.75281600 & 1.07905600 \\
\hline $\mathrm{N}$ & -2.48349700 & -1.18204700 & 1.56090300 \\
\hline $\mathrm{H}$ & -2.38344600 & -2.01558600 & 2.13173400 \\
\hline $\mathrm{C}$ & -3.79350000 & -0.79206600 & 1.19199100 \\
\hline $\mathrm{C}$ & -4.67978600 & -1.73889800 & 0.68583200 \\
\hline $\mathrm{C}$ & -4.23990400 & 0.52263400 & 1.36812000 \\
\hline $\mathrm{C}$ & -5.98751400 & -1.39563500 & 0.35238600 \\
\hline $\mathrm{H}$ & -4.33491100 & -2.76420100 & 0.54967200 \\
\hline $\mathrm{C}$ & -5.52767300 & 0.88070900 & 1.01211900 \\
\hline $\mathrm{H}$ & -3.56430900 & 1.26151600 & 1.79726300 \\
\hline $\mathrm{C}$ & -6.41343700 & -0.07596800 & 0.50537600 \\
\hline $\mathrm{H}$ & -6.65629900 & -2.16044100 & -0.03441000 \\
\hline $\mathrm{H}$ & -5.88684300 & 1.90013900 & 1.13712500 \\
\hline $\mathrm{O}$ & 3.08748200 & 0.38928800 & -3.81430100 \\
\hline $\mathrm{C}$ & -0.17340000 & -2.12058900 & -1.50370400 \\
\hline $\mathrm{C}$ & -1.54407300 & -2.40150200 & -1.53827300 \\
\hline $\mathrm{C}$ & 0.72329200 & -3.19259800 & -1.43948100 \\
\hline $\mathrm{C}$ & -2.00304400 & -3.71331500 & -1.49276000 \\
\hline $\mathrm{H}$ & -2.25860300 & -1.58202100 & -1.62556100 \\
\hline $\mathrm{C}$ & 0.26574800 & -4.50251400 & -1.39391100 \\
\hline $\mathrm{H}$ & 1.79267400 & -2.98657300 & -1.39329500 \\
\hline $\mathrm{C}$ & -1.10081200 & -4.76956100 & -1.41874600 \\
\hline $\mathrm{H}$ & -3.07428900 & -3.90981700 & -1.53388800 \\
\hline $\mathrm{H}$ & 0.98059000 & -5.32182500 & -1.33265000 \\
\hline $\mathrm{H}$ & -1.45934800 & -5.79711000 & -1.38578200 \\
\hline $\mathrm{C}$ & 0.22928600 & 2.99278000 & -1.09212600 \\
\hline
\end{tabular}




\begin{tabular}{lrrr}
$\mathrm{C}$ & 1.25408500 & 3.93329200 & -0.92065900 \\
$\mathrm{C}$ & -1.09545400 & 3.44242000 & -1.05186800 \\
$\mathrm{C}$ & 0.96325500 & 5.27458900 & -0.71405800 \\
$\mathrm{H}$ & 2.28804700 & 3.58657600 & -0.91210600 \\
$\mathrm{C}$ & -1.38732700 & 4.78538300 & -0.83275300 \\
$\mathrm{H}$ & -1.90720700 & 2.73414200 & -1.21658000 \\
$\mathrm{C}$ & -0.36035200 & 5.70692900 & -0.66379000 \\
$\mathrm{H}$ & 1.77583100 & 5.98635900 & -0.57641900 \\
$\mathrm{H}$ & -2.42580700 & 5.11251800 & -0.80424800 \\
$\mathrm{H}$ & -0.58821800 & 6.75763200 & -0.49212200 \\
$\mathrm{O}$ & -7.65479200 & 0.37316800 & 0.20046400 \\
$\mathrm{C}$ & -8.58046800 & -0.55254400 & -0.31004500 \\
$\mathrm{H}$ & -9.50487700 & 0.00212800 & -0.49001700 \\
$\mathrm{H}$ & -8.23406800 & -0.99166100 & -1.25813600 \\
$\mathrm{H}$ & -8.78121100 & -1.36310100 & 0.40719600 \\
$\mathrm{C}$ & 3.25720300 & -1.04961400 & 2.01621900 \\
$\mathrm{C}$ & 3.97713300 & -2.02364600 & 2.71593600 \\
$\mathrm{C}$ & 4.67601500 & -2.99642900 & 2.02430700 \\
$\mathrm{H}$ & 3.98327800 & -2.00767700 & 3.80328400 \\
$\mathrm{C}$ & 3.93962000 & -1.99438300 & -0.00739900 \\
$\mathrm{C}$ & 4.66790700 & -2.98323500 & 0.63021900 \\
$\mathrm{H}$ & 5.23490300 & -3.75741900 & 2.56603500 \\
$\mathrm{H}$ & 3.91114100 & -1.91441100 & -1.09417100 \\
$\mathrm{H}$ & 5.21893800 & -3.71645300 & 0.04733500 \\
$\mathrm{~N}$ & 3.23973400 & -1.06930000 & 0.65984300 \\
$\mathrm{O}$ & -0.24225500 & -1.28990600 & 1.46134600 \\
$\mathrm{C}$ & 1.90562000 & 0.93056700 & 1.68067800 \\
$\mathrm{H}$ & 3.29672300 & 1.32697900 & -0.12496300 \\
& & & \\
\hline
\end{tabular}

\begin{tabular}{|c|c|c|c|}
\hline $\mathrm{C}$ & -5.79565100 & 1.85976000 & -1.06531900 \\
\hline $\mathrm{H}$ & -4.22814900 & 3.29152600 & -1.43249300 \\
\hline $\mathrm{C}$ & -6.25145200 & 0.83341600 & -0.23262700 \\
\hline $\mathrm{H}$ & -5.78960800 & -0.40791500 & 1.48241300 \\
\hline $\mathrm{H}$ & -6.43027200 & 2.17694200 & -1.89038500 \\
\hline $\mathrm{O}$ & 1.92829800 & -3.28110200 & -1.49586300 \\
\hline $\mathrm{C}$ & -2.26028000 & -1.37357100 & -0.77657600 \\
\hline $\mathrm{C}$ & -3.29017200 & -1.07400300 & -1.67241900 \\
\hline $\mathrm{C}$ & -2.49248900 & -2.28915500 & 0.25766600 \\
\hline $\mathrm{C}$ & -4.52915600 & -1.69451900 & -1.55057100 \\
\hline $\mathrm{H}$ & -3.11170600 & -0.37684600 & -2.49203300 \\
\hline $\mathrm{C}$ & -3.73135200 & -2.90705200 & 0.37426100 \\
\hline $\mathrm{H}$ & -1.67930600 & -2.50873900 & 0.95504300 \\
\hline $\mathrm{C}$ & -4.74668500 & -2.62147400 & -0.53599900 \\
\hline $\mathrm{H}$ & -5.32512900 & -1.45763800 & -2.25601700 \\
\hline $\mathrm{H}$ & -3.90051600 & -3.62699300 & 1.17381300 \\
\hline $\mathrm{H}$ & -5.70844500 & -3.12899100 & -0.45818900 \\
\hline $\mathrm{C}$ & 2.03135900 & 1.37354900 & -1.90053000 \\
\hline $\mathrm{C}$ & 3.01592900 & 1.05546900 & -2.84787600 \\
\hline $\mathrm{C}$ & 2.07198700 & 2.62975600 & -1.28434300 \\
\hline $\mathrm{C}$ & 4.01480600 & 1.96362500 & -3.17117500 \\
\hline $\mathrm{H}$ & 2.99015400 & 0.08830900 & -3.34903100 \\
\hline $\mathrm{C}$ & 3.07987300 & 3.53218900 & -1.60618900 \\
\hline $\mathrm{H}$ & 1.32509400 & 2.88813200 & -0.53321800 \\
\hline $\mathrm{C}$ & 4.05430700 & 3.20595600 & -2.54420100 \\
\hline $\mathrm{H}$ & 4.76646500 & 1.69752200 & -3.91268700 \\
\hline $\mathrm{H}$ & 3.10434000 & 4.50013000 & -1.10749300 \\
\hline $\mathrm{H}$ & 4.84228900 & 3.91704800 & -2.78814400 \\
\hline $\mathrm{O}$ & -7.45954500 & 0.30829800 & -0.55597700 \\
\hline $\mathrm{C}$ & -7.98700600 & -0.68713600 & 0.28443000 \\
\hline $\mathrm{H}$ & -8.96309600 & -0.95509900 & -0.12857200 \\
\hline $\mathrm{H}$ & -7.34546500 & -1.58154500 & 0.30581700 \\
\hline $\mathrm{H}$ & -8.11888000 & -0.31976300 & 1.31363500 \\
\hline $\mathrm{C}$ & 3.51692100 & 0.60542200 & 1.42592900 \\
\hline $\mathrm{C}$ & 4.33297100 & 1.62156500 & 1.93667400 \\
\hline $\mathrm{C}$ & 3.77713700 & 2.69469300 & 2.61402000 \\
\hline $\mathrm{H}$ & 5.41056500 & 1.54025200 & 1.81768300 \\
\hline $\mathrm{C}$ & 1.64651600 & 1.68356900 & 2.27571200 \\
\hline $\mathrm{C}$ & 2.40261300 & 2.72505900 & 2.79166800 \\
\hline $\mathrm{H}$ & 4.41349700 & 3.48654600 & 3.00614400 \\
\hline $\mathrm{H}$ & 0.55753900 & 1.66242800 & 2.34261700 \\
\hline $\mathrm{H}$ & 1.90593100 & 3.54050800 & 3.31105800 \\
\hline $\mathrm{N}$ & 2.17672700 & 0.64936000 & 1.60282500 \\
\hline $\mathrm{O}$ & -0.35521000 & 3.08862500 & 0.53459300 \\
\hline $\mathrm{C}$ & 3.78061800 & -1.85232700 & 0.95088800 \\
\hline $\mathrm{H}$ & 2.86574400 & -2.06885800 & 1.49794400 \\
\hline $\mathrm{C}$ & -1.24112200 & -0.13216300 & 2.90676800 \\
\hline $\mathrm{H}$ & -1.63455000 & -1.16467700 & 2.81536400 \\
\hline $\mathrm{H}$ & -0.45701400 & -0.14775800 & 3.69239900 \\
\hline $\mathrm{H}$ & -2.06803500 & 0.48864700 & 3.31285500 \\
\hline $\mathrm{C}$ & 1.00897300 & -2.22338500 & 3.00434400 \\
\hline $\mathrm{H}$ & 1.68517100 & -3.07899100 & 3.20634400 \\
\hline $\mathrm{H}$ & 1.54972300 & -1.31612300 & 3.34196400 \\
\hline $\mathrm{H}$ & 0.13880800 & -2.34248700 & 3.67816700 \\
\hline $\mathrm{O}$ & 0.66769100 & -2.19917200 & 1.66649400 \\
\hline $\mathrm{O}$ & -0.82387800 & 0.41441100 & 1.71766800 \\
\hline
\end{tabular}

\section{INT1'}

Sum of electronic and zero-point Energies = $-2178.731298$

Sum of electronic and thermal Free Energies = $-2178.806346$

$\begin{array}{crrr}\text { Rh } & 0.57025000 & -0.50274800 & 0.48858500 \\ \mathrm{C} & 4.17385200 & -0.52714200 & 0.73742900 \\ \mathrm{C} & 4.52151200 & -2.89752100 & 0.40808000 \\ \mathrm{C} & -0.36771000 & 0.46099300 & -1.24805800 \\ \mathrm{C} & 1.03482000 & 0.34388700 & -1.56458400 \\ \mathrm{C} & 1.35302600 & -1.06136600 & -1.57277400 \\ \mathrm{C} & 0.20367600 & -1.78691900 & -1.24178800 \\ \mathrm{C} & -0.88793400 & -0.89138300 & -1.00720700 \\ \mathrm{C} & -1.20016800 & 1.71127400 & -1.25928300 \\ \mathrm{C} & 5.28685700 & -0.27486900 & -0.07718500 \\ \mathrm{C} & 6.01421100 & -1.32120800 & -0.63058300 \\ \mathrm{C} & 5.64531100 & -2.63912800 & -0.37031200 \\ \mathrm{C} & 2.40315500 & -2.03597100 & -1.98586700 \\ \mathrm{H} & 4.19523700 & -3.92185100 & 0.57643000 \\ \mathrm{H} & 5.56804600 & 0.75556300 & -0.29629000 \\ \mathrm{H} & 6.21891000 & -3.46185400 & -0.79434200 \\ \mathrm{H} & 6.87114100 & -1.10624000 & -1.26726800 \\ \mathrm{H} & -0.75946000 & 2.40951800 & -1.98740500 \\ \mathrm{H} & 2.48161800 & -2.06516400 & -3.09146800 \\ \mathrm{H} & -2.20358900 & 1.45630400 & -1.62068300 \\ \mathrm{H} & 3.40509900 & -1.85198600 & -1.58191200 \\ \mathrm{C} & 0.53339600 & -3.23378600 & -1.20104600 \\ \mathrm{H} & -0.04860200 & -3.81042200 & -1.94096800 \\ \mathrm{H} & 0.37379800 & -3.65697300 & -0.19765800 \\ \mathrm{C} & -1.31222100 & 2.51529800 & 0.03707900 \\ \mathrm{~N} & -2.56180300 & 2.70386900 & 0.55487200 \\ \mathrm{H} & -2.51242000 & 3.18563500 & 1.44796300 \\ \mathrm{C} & -3.77955200 & 2.03348600 & 0.25016000 \\ \mathrm{C} & -4.22607100 & 0.99382300 & 1.05939500 \\ \mathrm{C} & -4.57612700 & 2.46510100 & -0.81308800 \\ \mathrm{C} & -5.46624500 & 0.40559500 & 0.83828300 \\ \mathrm{H} & -3.58410900 & 0.63802300 & 1.86394900\end{array}$

\section{TS1'}

Sum of electronic and zero-point Energies = $-2178.678107$

Sum of electronic and thermal Free Energies $=$ $-2178.754664$

$\begin{array}{cccc}\mathrm{Rh} & 0.64984100 & -0.32140600 & 0.30736100 \\ \mathrm{C} & 3.82468100 & -1.05704700 & 0.92523800 \\ \mathrm{C} & 3.35312300 & -3.32002800 & 0.33539000\end{array}$




$\begin{array}{lrrr}\mathrm{C} & 1.80026400 & 1.72321600 & 2.22201700 \\ \mathrm{C} & 2.70815500 & 2.54333500 & 2.86963400 \\ \mathrm{H} & 4.79915400 & 2.78477900 & 3.35806600 \\ \mathrm{H} & 0.73475700 & 1.94100900 & 2.19507500 \\ \mathrm{H} & 2.36037600 & 3.44373300 & 3.36907100 \\ \mathrm{~N} & 2.16516300 & 0.61004000 & 1.57068600 \\ \mathrm{O} & -0.29754400 & 3.30465500 & 0.78116000 \\ \mathrm{C} & 2.88687500 & -2.10700500 & 0.85962200 \\ \mathrm{H} & 1.49813400 & -2.39320200 & 1.19215900 \\ \mathrm{C} & -0.64209600 & -0.38894600 & 2.94001300 \\ \mathrm{H} & -1.18248900 & -1.35180900 & 2.87444700 \\ \mathrm{H} & 0.37133200 & -0.61270900 & 3.32792800 \\ \mathrm{H} & -1.15259000 & 0.23140500 & 3.70310600 \\ \mathrm{C} & 0.34974000 & -3.47687900 & 2.49617000 \\ \mathrm{H} & 0.71665000 & -4.51611800 & 2.39598800 \\ \mathrm{H} & 0.95180200 & -2.99826200 & 3.29373500 \\ \mathrm{H} & -0.69186900 & -3.53288200 & 2.85498500 \\ \mathrm{O} & 0.42652200 & -2.78390400 & 1.29329400 \\ \mathrm{O} & -0.63033700 & 0.30376200 & 1.74376100\end{array}$

\section{INT2,}

Sum of electronic and zero-point Energies = $-2178.757032$

Sum of electronic and thermal Free Energies = $-2178.835003$

$\begin{array}{crrr}\mathrm{Rh} & 0.69961300 & 0.51537500 & 0.00434400 \\ \mathrm{C} & 3.05716100 & 1.53354400 & -1.38568900 \\ \mathrm{C} & 2.09161700 & 3.22349200 & 0.04040300 \\ \mathrm{C} & -0.21317500 & -1.23538100 & 1.45640800 \\ \mathrm{C} & 1.23194400 & -0.96681500 & 1.61315000 \\ \mathrm{C} & 1.34361300 & 0.36879100 & 2.08537700 \\ \mathrm{C} & 0.06442500 & 0.97325800 & 2.05219800 \\ \mathrm{C} & -0.91674700 & -0.04542400 & 1.75619500 \\ \mathrm{C} & -0.88684000 & -2.54432800 & 1.13448900 \\ \mathrm{C} & 4.08385000 & 2.41680500 & -1.74200700 \\ \mathrm{C} & 4.11919700 & 3.69402200 & -1.20484100 \\ \mathrm{C} & 3.11614800 & 4.09598900 & -0.32412600 \\ \mathrm{C} & 2.28763100 & 1.29160000 & 2.78165800 \\ \mathrm{H} & 1.30972100 & 3.56840100 & 0.71352700 \\ \mathrm{H} & 4.84679400 & 2.11330500 & -2.45956500 \\ \mathrm{H} & 3.12579400 & 5.10586700 & 0.08562800 \\ \mathrm{H} & 4.91564800 & 4.38179200 & -1.48340800 \\ \mathrm{H} & -0.69856000 & -3.26306000 & 1.94735800 \\ \mathrm{H} & 2.60754300 & 0.85467800 & 3.74549300 \\ \mathrm{H} & -1.96650000 & -2.37348300 & 1.10584800 \\ \mathrm{H} & 3.19026800 & 1.55327900 & 2.21222500 \\ \mathrm{C} & 0.13533700 & 2.27233100 & 2.78220600 \\ \mathrm{H} & -0.39666800 & 2.20593300 & 3.74964500 \\ \mathrm{H} & -0.26494400 & 3.13077800 & 2.22363900 \\ \mathrm{C} & -0.49230000 & -3.27257600 & -0.13843500 \\ \mathrm{~N} & -1.39890200 & -3.20529300 & -1.16895400 \\ \mathrm{H} & -1.14461400 & -3.79943400 & -1.95335300 \\ \mathrm{C} & -2.58214400 & -2.41185500 & -1.26139300 \\ \mathrm{C} & -2.49644600 & -1.03231700 & -1.41216900 \\ \mathrm{C} & -3.83957500 & -3.01963100 & -1.20149000 \\ \mathrm{C} & -3.64579400 & -0.25355200 & -1.49618000 \\ \mathrm{H} & -1.53065100 & -0.52362800 & -1.43717700 \\ \mathrm{C} & -4.98836300 & -2.25467500 & -1.29874500 \\ \mathrm{H} & -3.90520000 & -4.09993100 & -1.07548000 \\ \mathrm{C} & -4.89857800 & -0.86485600 & -1.44492500 \\ \mathrm{H} & -3.52993200 & 0.82483200 & -1.57842700 \\ \mathrm{H} & -5.97761100 & -2.70603900 & -1.25749700 \\ \mathrm{C} & 1.53281000 & 2.47861700 & 2.98666200 \\ \mathrm{C} & -2.36258100 & 0.21436200 & 1.79688600 \\ & -3.26678100 & -0.71391900 & 2.33225000\end{array}$




\begin{tabular}{|c|c|c|c|}
\hline 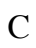 & -2.85376000 & 1.45730600 & 1.37376700 \\
\hline 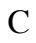 & -4.62640700 & -0.43317100 & 2.38938900 \\
\hline $\mathrm{H}$ & -2.90164100 & -1.65161000 & 2.74803200 \\
\hline $\mathrm{C}$ & -4.21149200 & 1.74034800 & 1.45207600 \\
\hline H & -2.17971600 & 2.20791500 & 0.96364700 \\
\hline $\mathrm{C}$ & -5.10658600 & 0.79340800 & 1.94190500 \\
\hline $\mathrm{H}$ & -5.31093600 & -1.17198100 & 2.80261600 \\
\hline $\mathrm{H}$ & -4.56820100 & 2.71737300 & 1.12648800 \\
\hline 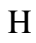 & -6.17206900 & 1.01480400 & 1.98795000 \\
\hline $\mathrm{C}$ & 2.37109300 & -1.90037400 & 1.54587400 \\
\hline $\mathrm{C}$ & 2.29333800 & -3.19704200 & 2.05952400 \\
\hline $\mathrm{C}$ & 3.59546500 & -1.45774900 & 1.02969300 \\
\hline $\mathrm{C}$ & 3.40430700 & -4.03073100 & 2.04924700 \\
\hline $\mathrm{H}$ & 1.35619500 & -3.55839600 & 2.47713700 \\
\hline $\mathrm{C}$ & 4.70414600 & -2.29316100 & 1.01205800 \\
\hline $\mathrm{H}$ & 3.66761200 & -0.44771000 & 0.62383500 \\
\hline $\mathrm{C}$ & 4.61163400 & -3.58520500 & 1.52181600 \\
\hline H & 3.32246400 & -5.03857200 & 2.45247800 \\
\hline $\mathrm{H}$ & 5.64408200 & -1.93253800 & 0.59658000 \\
\hline $\mathrm{H}$ & 5.47887300 & -4.24345700 & 1.50810500 \\
\hline $\mathrm{O}$ & -6.07908700 & -0.20710100 & -1.52474800 \\
\hline $\mathrm{C}$ & -6.03857000 & 1.18424900 & -1.73184600 \\
\hline $\mathrm{H}$ & -7.07835600 & 1.51576200 & -1.79747300 \\
\hline $\mathrm{H}$ & -5.54297700 & 1.70127000 & -0.89615000 \\
\hline H & -5.51827600 & 1.43694600 & -2.66810300 \\
\hline $\mathrm{C}$ & 2.89461400 & 0.21253500 & -1.97792900 \\
\hline $\mathrm{C}$ & 3.80875400 & -0.41529700 & -2.82589900 \\
\hline $\mathrm{C}$ & 3.52283600 & -1.67217900 & -3.33448300 \\
\hline $\mathrm{H}$ & 4.74591600 & 0.07968200 & -3.06953300 \\
\hline $\mathrm{C}$ & 1.44025900 & -1.59099600 & -2.18119700 \\
\hline $\mathrm{C}$ & 2.30848700 & -2.27142800 & -3.01939400 \\
\hline $\mathrm{H}$ & 4.24045600 & -2.17798100 & -3.97788200 \\
\hline $\mathrm{H}$ & 0.46810600 & -2.00160900 & -1.92785300 \\
\hline $\mathrm{H}$ & 2.03362500 & -3.25298200 & -3.39577800 \\
\hline $\mathrm{N}$ & 1.73205000 & -0.40175500 & -1.64869700 \\
\hline $\mathrm{O}$ & 0.53293800 & -3.92220000 & -0.25333300 \\
\hline $\mathrm{C}$ & 2.06455300 & 1.92686500 & -0.46669200 \\
\hline $\mathrm{H}$ & -0.83715300 & 3.02116400 & -0.39522100 \\
\hline $\mathrm{C}$ & -0.38881600 & 1.74463200 & -2.54361600 \\
\hline $\mathrm{H}$ & 0.55347100 & 2.29552500 & -2.73316800 \\
\hline $\mathrm{H}$ & -0.33752900 & 0.79831600 & -3.12013500 \\
\hline $\mathrm{H}$ & -1.20841100 & 2.34281400 & -2.98452400 \\
\hline $\mathrm{C}$ & -0.82474400 & 4.93125400 & -0.76606600 \\
\hline $\mathrm{H}$ & 0.21162600 & 4.99144500 & -1.14673100 \\
\hline $\mathrm{H}$ & -1.50066100 & 4.88779800 & -1.63838500 \\
\hline $\mathrm{H}$ & -1.03841800 & 5.86250600 & -0.22631000 \\
\hline $\mathrm{O}$ & -1.00788500 & 3.85915200 & 0.11217900 \\
\hline $\mathrm{O}$ & -0.65228300 & 1.54339600 & -1.19784600 \\
\hline
\end{tabular}

\section{INT3,}

Sum of electronic and zero-point Energies= 2063.141067

Sum of electronic and thermal Free Energies= 2063.215211

$\begin{array}{crrr}\text { Rh } & 0.69503000 & -0.67471100 & 0.01044000 \\ \mathrm{C} & 2.97195100 & -1.73155200 & 1.49264100 \\ \mathrm{C} & 1.97802000 & -3.42287500 & 0.09113500 \\ \mathrm{C} & -0.24356500 & 1.00747300 & -1.48990100 \\ \mathrm{C} & 1.21152100 & 0.82266600 & -1.63102200 \\ \mathrm{C} & 1.41177000 & -0.51381200 & -2.06446700 \\ \mathrm{C} & 0.16782000 & -1.18929100 & -2.05000500 \\ \mathrm{C} & -0.87009400 & -0.23523100 & -1.75867200 \\ \mathrm{C} & -1.00460900 & 2.27787700 & -1.21571600 \\ \mathrm{C} & 3.94743400 & -2.64596600 & 1.90824300 \\ \mathrm{C} & 3.94425800 & -3.93893600 & 1.40810200 \\ \mathrm{C} & 2.95129000 & -4.32737500 & 0.50911400\end{array}$

\begin{tabular}{|c|c|c|c|}
\hline $\mathrm{C}$ & 2.42910700 & -1.40225700 & -2.69875600 \\
\hline H & 1.20195600 & -3.75321500 & -0.59719700 \\
\hline $\mathrm{H}$ & 4.69892400 & -2.35495100 & 2.64304700 \\
\hline H & 2.93164700 & -5.34942400 & 0.13183700 \\
\hline $\mathrm{H}$ & 4.70156500 & -4.65098400 & 1.73111400 \\
\hline $\mathrm{H}$ & -0.84353500 & 2.98800900 & -2.04175100 \\
\hline H & 2.78296000 & -0.97465200 & -3.65383700 \\
\hline H & -2.07245100 & 2.03694200 & -1.20751400 \\
\hline $\mathrm{H}$ & 3.30854700 & -1.61682000 & -2.07569300 \\
\hline $\mathrm{C}$ & 0.33018900 & -2.48754100 & -2.76258600 \\
\hline $\mathrm{H}$ & -0.17968100 & -2.46854800 & -3.74352800 \\
\hline $\mathrm{H}$ & -0.04455400 & -3.36555800 & -2.21486200 \\
\hline $\mathrm{C}$ & -0.66293500 & 3.04907400 & 0.04664500 \\
\hline $\mathrm{N}$ & -1.56644600 & 2.95611300 & 1.07510000 \\
\hline $\mathrm{H}$ & -1.33362000 & 3.56458200 & 1.85501000 \\
\hline $\mathrm{C}$ & -2.73747900 & 2.14461800 & 1.16495700 \\
\hline $\mathrm{C}$ & -2.63131500 & 0.77748000 & 1.39276000 \\
\hline $\mathrm{C}$ & -4.00160600 & 2.72635100 & 1.03259000 \\
\hline $\mathrm{C}$ & -3.77180100 & -0.01191000 & 1.49828200 \\
\hline $\mathrm{H}$ & -1.65688300 & 0.28785500 & 1.45766900 \\
\hline $\mathrm{C}$ & -5.14086900 & 1.94859900 & 1.13876500 \\
\hline $\mathrm{H}$ & -4.07947000 & 3.79686100 & 0.84494800 \\
\hline $\mathrm{C}$ & -5.03192900 & 0.57321000 & 1.37644000 \\
\hline $\mathrm{H}$ & -3.64533500 & -1.08082800 & 1.65297300 \\
\hline $\mathrm{H}$ & -6.13569500 & 2.37882300 & 1.04246400 \\
\hline $\mathrm{O}$ & 1.73653500 & -2.62706400 & -2.92440900 \\
\hline $\mathrm{C}$ & -2.29226300 & -0.60481200 & -1.74536100 \\
\hline $\mathrm{C}$ & -3.27016500 & 0.18447500 & -2.36317200 \\
\hline $\mathrm{C}$ & -2.67193200 & -1.82517600 & -1.16877100 \\
\hline $\mathrm{C}$ & -4.59891700 & -0.22272300 & -2.38561100 \\
\hline $\mathrm{H}$ & -2.98362300 & 1.10779700 & -2.86571800 \\
\hline $\mathrm{C}$ & -3.99858500 & -2.23547100 & -1.21151400 \\
\hline $\mathrm{H}$ & -1.92594200 & -2.41076900 & -0.63043200 \\
\hline $\mathrm{C}$ & -4.96745200 & -1.43596900 & -1.81276500 \\
\hline $\mathrm{H}$ & -5.34662200 & 0.40410300 & -2.86852100 \\
\hline $\mathrm{H}$ & -4.27875800 & -3.18934700 & -0.76401900 \\
\hline $\mathrm{H}$ & -6.00796500 & -1.75769200 & -1.83586600 \\
\hline $\mathrm{C}$ & 2.29461300 & 1.81897600 & -1.54824100 \\
\hline $\mathrm{C}$ & 2.16391300 & 3.09178900 & -2.10842400 \\
\hline $\mathrm{C}$ & 3.51807000 & 1.46636800 & -0.96587900 \\
\hline $\mathrm{C}$ & 3.22246800 & 3.99037200 & -2.07977700 \\
\hline $\mathrm{H}$ & 1.22530800 & 3.38200600 & -2.57607000 \\
\hline $\mathrm{C}$ & 4.57480300 & 2.36687300 & -0.93029100 \\
\hline $\mathrm{H}$ & 3.62943000 & 0.47601900 & -0.52184600 \\
\hline $\mathrm{C}$ & 4.42949100 & 3.63415700 & -1.48717100 \\
\hline $\mathrm{H}$ & 3.10008700 & 4.97872200 & -2.51942200 \\
\hline $\mathrm{H}$ & 5.51469700 & 2.07695600 & -0.46261900 \\
\hline $\mathrm{H}$ & 5.25522400 & 4.34337200 & -1.45865800 \\
\hline $\mathrm{O}$ & -6.20413300 & -0.09982500 & 1.46886300 \\
\hline $\mathrm{C}$ & -6.14764900 & -1.46154000 & 1.81564200 \\
\hline $\mathrm{H}$ & -7.18304400 & -1.80730200 & 1.87803500 \\
\hline $\mathrm{H}$ & -5.61290600 & -2.05001900 & 1.05535800 \\
\hline $\mathrm{H}$ & -5.65658600 & -1.60869900 & 2.78958100 \\
\hline $\mathrm{C}$ & 2.83238300 & -0.39019200 & 2.04450400 \\
\hline $\mathrm{C}$ & 3.73099000 & 0.23154900 & 2.91312500 \\
\hline $\mathrm{C}$ & 3.46677500 & 1.51157600 & 3.37390200 \\
\hline $\mathrm{H}$ & 4.63896100 & -0.28789300 & 3.21099800 \\
\hline $\mathrm{C}$ & 1.43457000 & 1.47065000 & 2.13010200 \\
\hline $\mathrm{C}$ & 2.29067400 & 2.14439500 & 2.98608900 \\
\hline $\mathrm{H}$ & 4.17219000 & 2.01063800 & 4.03586700 \\
\hline $\mathrm{H}$ & 0.49392000 & 1.91004600 & 1.81397000 \\
\hline $\mathrm{H}$ & 2.03620700 & 3.14553400 & 3.32286500 \\
\hline $\mathrm{N}$ & 1.70524200 & 0.25338300 & 1.65082300 \\
\hline $\mathrm{O}$ & 0.33429300 & 3.74316900 & 0.15686400 \\
\hline $\mathrm{C}$ & 1.99500100 & -2.11055200 & 0.55199900 \\
\hline & -0.45713800 & -1.88269300 & 2.50487800 \\
\hline
\end{tabular}


H $\quad 0.46483000 \quad-2.45410500 \quad 2.73195800$

$\mathrm{H} \quad-0.40714600 \quad-0.93721300 \quad 3.08959700$

$\mathrm{H} \quad-1.29953700 \quad-2.46712700 \quad 2.92108000$

$\begin{array}{llll}\mathrm{O} & -0.67818200 & -1.68922000 & 1.15706200\end{array}$

\section{INT3-Rh(III)}

Sum of electronic and zero-point Energies = $-2456.310194$

Sum of electronic and thermal Free Energies = $-2456.396295$

\begin{tabular}{|c|c|c|c|}
\hline $\mathrm{Rh}$ & -1.08582900 & 0.24701900 & -0.14698800 \\
\hline $\mathrm{C}$ & -1.47331100 & -0.04922200 & 2.72675300 \\
\hline $\mathrm{C}$ & -0.37559800 & -1.98997300 & 1.82047900 \\
\hline $\mathrm{C}$ & 1.10113200 & 0.02764600 & -0.96298700 \\
\hline $\mathrm{C}$ & 0.13798800 & -0.88104600 & -1.58489300 \\
\hline $\mathrm{C}$ & -0.74229600 & -0.04768600 & -2.35598700 \\
\hline $\mathrm{C}$ & -0.41433700 & 1.29050800 & -2.13468300 \\
\hline $\mathrm{C}$ & 0.75808200 & 1.36085800 & -1.30312800 \\
\hline $\mathrm{C}$ & 2.19677700 & -0.39624900 & -0.03626500 \\
\hline $\mathrm{C}$ & -1.35967900 & -0.58304700 & 4.01388400 \\
\hline $\mathrm{C}$ & -0.74853100 & -1.81423000 & 4.20823600 \\
\hline $\mathrm{C}$ & -0.26350900 & -2.51814500 & 3.10597200 \\
\hline $\mathrm{C}$ & -1.79816600 & -0.11634300 & -3.40626200 \\
\hline $\mathrm{H}$ & -0.00955400 & -2.57678500 & 0.97405000 \\
\hline $\mathrm{H}$ & -1.74562100 & -0.03515700 & 4.87438900 \\
\hline $\mathrm{H}$ & 0.20505300 & -3.49244800 & 3.24857700 \\
\hline $\mathrm{H}$ & -0.65500500 & -2.22689500 & 5.21138000 \\
\hline $\mathrm{H}$ & 1.95056700 & -1.39297000 & 0.36668500 \\
\hline $\mathrm{H}$ & -1.37795500 & -0.48075400 & -4.36211000 \\
\hline $\mathrm{H}$ & 3.14461100 & -0.52515300 & -0.58001800 \\
\hline $\mathrm{H}$ & -2.67645800 & -0.72518300 & -3.15638600 \\
\hline $\mathrm{C}$ & -1.23736100 & 2.13774700 & -3.04540000 \\
\hline $\mathrm{H}$ & -0.63204600 & 2.55236800 & -3.87402100 \\
\hline $\mathrm{H}$ & -1.75451200 & 2.98442800 & -2.56658700 \\
\hline $\mathrm{C}$ & 2.41192300 & 0.51545700 & 1.16529700 \\
\hline $\mathrm{N}$ & 3.70613500 & 0.61456200 & 1.63065400 \\
\hline $\mathrm{H}$ & 3.78526500 & 1.30152300 & 2.37387500 \\
\hline $\mathrm{C}$ & 4.90100500 & 0.06658800 & 1.10372500 \\
\hline $\mathrm{C}$ & 5.93139100 & 0.91018800 & 0.69843800 \\
\hline $\mathrm{C}$ & 5.08942600 & -1.31752900 & 1.01969400 \\
\hline $\mathrm{C}$ & 7.13258600 & 0.39788400 & 0.21450500 \\
\hline $\mathrm{H}$ & 5.78497300 & 1.98862800 & 0.76187300 \\
\hline $\mathrm{C}$ & 6.26705600 & -1.83660300 & 0.51304000 \\
\hline $\mathrm{H}$ & 4.29992000 & -1.98376600 & 1.36537100 \\
\hline $\mathrm{C}$ & 7.30022700 & -0.98328200 & 0.11131700 \\
\hline $\mathrm{H}$ & 7.92033400 & 1.08334900 & -0.08785700 \\
\hline $\mathrm{H}$ & 6.42620400 & -2.91016800 & 0.43573800 \\
\hline $\mathrm{O}$ & -2.22852400 & 1.24285700 & -3.54113100 \\
\hline $\mathrm{C}$ & 1.47201800 & 2.59187800 & -0.94343000 \\
\hline $\mathrm{C}$ & 2.86470800 & 2.66651800 & -1.05700600 \\
\hline $\mathrm{C}$ & 0.77519200 & 3.72990000 & -0.52277700 \\
\hline $\mathrm{C}$ & 3.54135100 & 3.84065900 & -0.74422800 \\
\hline $\mathrm{H}$ & 3.42074800 & 1.79996700 & -1.41763600 \\
\hline $\mathrm{C}$ & 1.45047600 & 4.90193000 & -0.21060000 \\
\hline $\mathrm{H}$ & -0.30772900 & 3.67531700 & -0.40649400 \\
\hline $\mathrm{C}$ & 2.83777200 & 4.96248700 & -0.31846200 \\
\hline $\mathrm{H}$ & 4.62492800 & 3.88353400 & -0.85272700 \\
\hline $\mathrm{H}$ & 0.89123300 & 5.77318600 & 0.12732900 \\
\hline $\mathrm{H}$ & 3.36664900 & 5.88264500 & -0.07529200 \\
\hline $\mathrm{C}$ & 0.17758700 & -2.35263800 & -1.62779000 \\
\hline $\mathrm{C}$ & -1.01162200 & -3.09339900 & -1.56003600 \\
\hline $\mathrm{C}$ & 1.38769200 & -3.04403000 & -1.74845000 \\
\hline $\mathrm{C}$ & -0.98431600 & -4.48057800 & -1.59968900 \\
\hline $\mathrm{H}$ & -1.96360600 & -2.57629700 & -1.42541900 \\
\hline $\mathrm{C}$ & 1.41350400 & -4.43571500 & -1.77774500 \\
\hline$U_{2}$ & 2.31916900 & -2.48472000 & 18360560 \\
\hline
\end{tabular}

$\begin{array}{ccc}0.22876200 & -5.15933100 & -1.70188100 \\ -1.92189000 & -5.03208200 & -1.53614500 \\ 2.36687900 & -4.95459900 & -1.86876200 \\ 0.24917200 & -6.24787100 & -1.72255300 \\ 8.41765700 & -1.59043800 & -0.35565400 \\ 9.48589500 & -0.77416400 & -0.76453900 \\ 10.27950400 & -1.44777800 & -1.09770700 \\ 9.19786500 & -0.11704500 & -1.59932700 \\ 9.86327400 & -0.15530900 & 0.06392500 \\ -2.12018200 & 1.22050100 & 2.43384200 \\ -2.77078300 & 2.04276900 & 3.35731700 \\ -3.37556100 & 3.21244300 & 2.93303600 \\ -2.80402200 & 1.75265000 & 4.40476600 \\ -2.68699200 & 2.69008000 & 0.71466100 \\ -3.33885100 & 3.54860000 & 1.58159300 \\ -3.88393600 & 3.85587400 & 3.64879900 \\ -2.64516600 & 2.88475800 & -0.35767000 \\ -3.81664300 & 4.44781900 & 1.20211000 \\ -2.08018800 & 1.57160300 & 1.12535300 \\ 1.51890400 & 1.11974900 & 1.72769900 \\ -0.96132100 & -0.73963300 & 1.60763300 \\ -2.43181600 & -0.56005200 & -0.16589600 \\ -4.06886900 & -2.54034200 & 2.34225400 \\ -3.61367700 & -3.45731800 & 1.19707500 \\ -3.26887000 & -2.34292300 & 3.06801200 \\ -4.94043000 & -2.93870300 & 2.88145900 \\ -2.52080800 & -3.58582800 & 1.18269700 \\ -4.08210500 & -4.44974300 & 1.22566200 \\ -5.21377700 & 0.54565800 & -2.83359500 \\ -5.05170500 & 1.65905900 & -1.80198400 \\ -4.59642100 & 0.70950200 & -3.72376900 \\ -6.26039600 & 0.40175400 & -3.14025200 \\ -4.10009900 & 2.19290400 & -1.95489800 \\ -5.87262000 & 2.38632200 & -1.80400400 \\ -4.39306900 & -1.51122300 & 0.35678200 \\ -4.73962300 & -0.33393700 & -0.80675200 \\ -4.44168100 & -1.31893800 & 1.71076300 \\ -4.00885000 & -2.78368400 & 0.00656300 \\ -4.77488500 & -0.62739700 & -2.15012300 \\ -5.00726300 & 0.98776900 & -0.54730700\end{array}$

\section{TS2-Rh(III)}

Sum of electronic and zero-point Energies = $-2456.249400$

Sum of electronic and thermal Free Energies = $-2456.335181$

$\begin{array}{crrr}\text { Rh } & -1.20762700 & 0.24694800 & -0.33857700 \\ \mathrm{C} & -1.49590000 & -0.00397400 & 2.57241700 \\ \mathrm{C} & -0.88056800 & -2.12948800 & 1.62616300 \\ \mathrm{C} & 1.07072900 & -0.06657300 & -1.05018800 \\ \mathrm{C} & 0.11036900 & -0.98158200 & -1.67391000 \\ \mathrm{C} & -0.70007200 & -0.17204000 & -2.52283800 \\ \mathrm{C} & -0.36045400 & 1.17538100 & -2.33743800 \\ \mathrm{C} & 0.80570300 & 1.24828600 & -1.49739100 \\ \mathrm{C} & 2.08200500 & -0.48084300 & -0.02622300 \\ \mathrm{C} & -1.49120400 & -0.56898100 & 3.85138400 \\ \mathrm{C} & -1.17577500 & -1.91067000 & 4.02117700 \\ \mathrm{C} & -0.87073300 & -2.68836000 & 2.90411500 \\ \mathrm{C} & -1.57531700 & -0.27923500 & -3.71995900 \\ \mathrm{H} & -0.66251600 & -2.77021300 & 0.76923600 \\ \mathrm{H} & -1.73543300 & 0.03822300 & 4.72430100 \\ \mathrm{H} & -0.62748200 & -3.74413700 & 3.02789400 \\ \mathrm{H} & -1.16855800 & -2.34918000 & 5.01807800 \\ \mathrm{H} & 1.77505800 & -1.45355500 & 0.39257400 \\ \mathrm{H} & -0.99351800 & -0.66030300 & -4.58111900 \\ \mathrm{H} & 3.06543200 & -0.65854400 & -0.48742300\end{array}$




\begin{tabular}{|c|c|c|c|}
\hline $\mathrm{H}$ & -2.46602200 & -0.90284300 & -3.59411400 \\
\hline $\mathrm{C}$ & -1.06675800 & 1.97930900 & -3.38172600 \\
\hline $\mathrm{H}$ & -0.36344700 & 2.35730500 & -4.14890200 \\
\hline $\mathrm{H}$ & -1.64136600 & 2.84166400 & -3.01002000 \\
\hline $\mathrm{C}$ & 2.23642600 & 0.46088400 & 1.15902000 \\
\hline $\mathrm{N}$ & 3.49345600 & 0.53413800 & 1.72571500 \\
\hline $\mathrm{H}$ & 3.53461200 & 1.24921700 & 2.44508300 \\
\hline $\mathrm{C}$ & 4.71494200 & -0.04135200 & 1.30195700 \\
\hline $\mathrm{C}$ & 5.80048100 & 0.77891900 & 1.00709900 \\
\hline $\mathrm{C}$ & 4.87613900 & -1.42900400 & 1.21547900 \\
\hline $\mathrm{C}$ & 7.02814500 & 0.24130700 & 0.62822300 \\
\hline $\mathrm{H}$ & 5.67770300 & 1.86018100 & 1.07494700 \\
\hline $\mathrm{C}$ & 6.08232600 & -1.97212300 & 0.81140500 \\
\hline $\mathrm{H}$ & 4.04271000 & -2.07964200 & 1.47742800 \\
\hline $\mathrm{C}$ & 7.16947000 & -1.14205200 & 0.51880200 \\
\hline $\mathrm{H}$ & 7.85726300 & 0.90997000 & 0.41072000 \\
\hline $\mathrm{H}$ & 6.22033700 & -3.04853800 & 0.73291300 \\
\hline $\mathrm{O}$ & -1.99728500 & 1.06395700 & -3.95619300 \\
\hline $\mathrm{C}$ & 1.53367400 & 2.47431600 & -1.17076700 \\
\hline $\mathrm{C}$ & 2.92960100 & 2.47599600 & -1.04723400 \\
\hline $\mathrm{C}$ & 0.85841200 & 3.69360600 & -1.03354000 \\
\hline $\mathrm{C}$ & 3.61998100 & 3.64923800 & -0.76937400 \\
\hline $\mathrm{H}$ & 3.48483900 & 1.54986700 & -1.19998600 \\
\hline $\mathrm{C}$ & 1.54799900 & 4.86625100 & -0.75550000 \\
\hline $\mathrm{H}$ & -0.22740600 & 3.70891200 & -1.12577400 \\
\hline $\mathrm{C}$ & 2.93339200 & 4.85032200 & -0.61775800 \\
\hline $\mathrm{H}$ & 4.70698200 & 3.62683000 & -0.69076900 \\
\hline $\mathrm{H}$ & 0.99914400 & 5.80036200 & -0.64291600 \\
\hline $\mathrm{H}$ & 3.47466800 & 5.77031100 & -0.40297800 \\
\hline $\mathrm{C}$ & 0.13189400 & -2.45216800 & -1.68990300 \\
\hline $\mathrm{C}$ & -1.07122800 & -3.16922300 & -1.78580800 \\
\hline $\mathrm{C}$ & 1.33086000 & -3.17295600 & -1.63945200 \\
\hline $\mathrm{C}$ & -1.06864400 & -4.55695400 & -1.81681900 \\
\hline $\mathrm{H}$ & -2.01719000 & -2.62348500 & -1.80021800 \\
\hline $\mathrm{C}$ & 1.32941700 & -4.56466400 & -1.65895000 \\
\hline $\mathrm{H}$ & 2.27979000 & -2.63825400 & -1.60706300 \\
\hline $\mathrm{C}$ & 0.13076100 & -5.26321000 & -1.74545300 \\
\hline $\mathrm{H}$ & -2.01493300 & -5.09202600 & -1.88248500 \\
\hline $\mathrm{H}$ & 2.27506000 & -5.10380700 & -1.61634000 \\
\hline $\mathrm{H}$ & 0.12925300 & -6.35206700 & -1.75950800 \\
\hline $\mathrm{O}$ & 8.30962600 & -1.77295000 & 0.14644100 \\
\hline $\mathrm{C}$ & 9.42828100 & -0.97925600 & -0.15773700 \\
\hline $\mathrm{H}$ & 10.23040200 & -1.66841500 & -0.43401900 \\
\hline $\mathrm{H}$ & 9.22804700 & -0.30290800 & -1.00286300 \\
\hline $\mathrm{H}$ & 9.74993400 & -0.38204800 & 0.70932800 \\
\hline $\mathrm{C}$ & -1.86029400 & 1.38312600 & 2.31073300 \\
\hline $\mathrm{C}$ & -2.16488900 & 2.34329300 & 3.27703500 \\
\hline $\mathrm{C}$ & -2.52822800 & 3.62087400 & 2.88676400 \\
\hline $\mathrm{H}$ & -2.10571400 & 2.08448300 & 4.33161400 \\
\hline $\mathrm{C}$ & -2.30271700 & 2.92525000 & 0.61952400 \\
\hline $\mathrm{C}$ & -2.60688500 & 3.92288800 & 1.52993200 \\
\hline $\mathrm{H}$ & -2.75798200 & 4.37698700 & 3.63550400 \\
\hline $\mathrm{H}$ & -2.38509800 & 3.08307400 & -0.45457500 \\
\hline $\mathrm{H}$ & -2.90678100 & 4.90705000 & 1.17963000 \\
\hline $\mathrm{N}$ & -1.91906500 & 1.70072500 & 0.99414500 \\
\hline $\mathrm{O}$ & 1.32492900 & 1.10988700 & 1.63513600 \\
\hline $\mathrm{C}$ & -1.18357700 & -0.77833300 & 1.43054000 \\
\hline $\mathrm{H}$ & -2.89948100 & -0.57660500 & 0.28470300 \\
\hline $\mathrm{C}$ & -5.11781100 & -1.32306300 & 2.79542800 \\
\hline $\mathrm{C}$ & -4.69038600 & -2.67585100 & 2.20044200 \\
\hline $\mathrm{H}$ & -4.67475800 & -1.13868000 & 3.78182400 \\
\hline $\mathrm{H}$ & -6.20775100 & -1.21360700 & 2.87157600 \\
\hline $\mathrm{H}$ & -3.84425500 & -3.11685600 & 2.74631600 \\
\hline $\mathrm{H}$ & -5.50565900 & -3.40701800 & 2.15487900 \\
\hline $\mathrm{C}$ & -4.85346200 & -0.29909300 & -2.51666500 \\
\hline $\mathrm{C}$ & -4.73668200 & 1.16399900 & -2.10441900 \\
\hline
\end{tabular}

$\begin{array}{lrrr}\text { H } & -4.75603200 & -0.44906400 & -3.60064000 \\ \mathrm{H} & -5.80990600 & -0.73935500 & -2.18463700 \\ \mathrm{H} & -4.04563500 & 1.69454500 & -2.78002500 \\ \mathrm{H} & -5.69966400 & 1.68957200 & -2.07995700 \\ \mathrm{~B} & -4.13266900 & -1.02524300 & 0.81442700 \\ \mathrm{~B} & -3.46796200 & -0.09912600 & -0.74924600 \\ \mathrm{O} & -4.61982500 & -0.34425700 & 1.88050700 \\ \mathrm{O} & -4.24899900 & -2.37393500 & 0.87419300 \\ \mathrm{O} & -3.78038300 & -0.93553400 & -1.84387800 \\ \mathrm{O} & -4.18626200 & 1.11215200 & -0.79904000\end{array}$

\section{INT4-Rh(III)}

Sum of electronic and zero-point Energies = -2456.325533

Sum of electronic and thermal Free Energies = -2456.411275

\begin{tabular}{|c|c|c|c|}
\hline $\mathrm{Rh}$ & -1.07011700 & -0.10732300 & -0.45314100 \\
\hline $\mathrm{C}$ & -1.64428900 & 0.42360800 & 2.35239100 \\
\hline $\mathrm{C}$ & -1.05822300 & -1.89118600 & 2.04578000 \\
\hline $\mathrm{C}$ & 1.29892800 & -0.47862500 & -1.04474600 \\
\hline $\mathrm{C}$ & 0.41437300 & -1.60479800 & -1.32648900 \\
\hline $\mathrm{C}$ & -0.47677700 & -1.15819700 & -2.35084500 \\
\hline $\mathrm{C}$ & -0.22379000 & 0.19589900 & -2.62362300 \\
\hline $\mathrm{C}$ & 0.92452200 & 0.61173400 & -1.86443500 \\
\hline $\mathrm{C}$ & 2.28381900 & -0.46068200 & 0.08361900 \\
\hline $\mathrm{C}$ & -1.75472200 & 0.21923700 & 3.73069800 \\
\hline $\mathrm{C}$ & -1.50843500 & -1.03525800 & 4.26800400 \\
\hline $\mathrm{C}$ & -1.16044000 & -2.08787500 & 3.42147800 \\
\hline $\mathrm{C}$ & -1.41439000 & -1.67633100 & -3.39085800 \\
\hline $\mathrm{H}$ & -0.79990500 & -2.73509900 & 1.40439000 \\
\hline $\mathrm{H}$ & -2.05245800 & 1.03809800 & 4.38675700 \\
\hline $\mathrm{H}$ & -0.97326000 & -3.07795800 & 3.83801500 \\
\hline $\mathrm{H}$ & -1.59745200 & -1.19789500 & 5.34092400 \\
\hline $\mathrm{H}$ & 2.06534900 & -1.31700500 & 0.74325400 \\
\hline $\mathrm{H}$ & -0.86220600 & -2.26311400 & -4.15068700 \\
\hline $\mathrm{H}$ & 3.31809600 & -0.61520200 & -0.25991100 \\
\hline $\mathrm{H}$ & -2.24478100 & -2.28874400 & -3.01971900 \\
\hline $\mathrm{C}$ & -1.03156300 & 0.57913700 & -3.82381800 \\
\hline $\mathrm{H}$ & -0.39827400 & 0.67244100 & -4.72835000 \\
\hline $\mathrm{H}$ & -1.61614500 & 1.50613000 & -3.73457800 \\
\hline $\mathrm{C}$ & 2.21724500 & 0.77993400 & 0.96392000 \\
\hline $\mathrm{N}$ & 3.38922700 & 1.16163600 & 1.57977300 \\
\hline $\mathrm{H}$ & 3.28131900 & 2.03659500 & 2.08305000 \\
\hline $\mathrm{C}$ & 4.69974200 & 0.64655400 & 1.42503400 \\
\hline $\mathrm{C}$ & 5.71583100 & 1.47321800 & 0.95407600 \\
\hline $\mathrm{C}$ & 5.01107900 & -0.67049900 & 1.78055100 \\
\hline $\mathrm{C}$ & 7.02316300 & 1.00912100 & 0.83182000 \\
\hline $\mathrm{H}$ & 5.47431700 & 2.49991900 & 0.67795700 \\
\hline $\mathrm{C}$ & 6.30030200 & -1.15004500 & 1.63588600 \\
\hline $\mathrm{H}$ & 4.22828200 & -1.31354400 & 2.18070500 \\
\hline $\mathrm{C}$ & 7.31773100 & -0.31309700 & 1.16529700 \\
\hline $\mathrm{H}$ & 7.79482100 & 1.68236200 & 0.46710900 \\
\hline $\mathrm{H}$ & 6.55696900 & -2.17325900 & 1.90226500 \\
\hline $\mathrm{O}$ & -1.95907400 & -0.49267800 & -3.96776400 \\
\hline $\mathrm{C}$ & 1.55549800 & 1.93137200 & -1.94442400 \\
\hline $\mathrm{C}$ & 2.93221000 & 2.09283600 & -1.73850700 \\
\hline $\mathrm{C}$ & 0.80798400 & 3.06254700 & -2.29490100 \\
\hline $\mathrm{C}$ & 3.52998600 & 3.34196300 & -1.84975900 \\
\hline $\mathrm{H}$ & 3.55157100 & 1.22337100 & -1.51867200 \\
\hline $\mathrm{C}$ & 1.40369600 & 4.31219500 & -2.40369000 \\
\hline $\mathrm{H}$ & -0.26115200 & 2.95471300 & -2.47328500 \\
\hline $\mathrm{C}$ & 2.76887500 & 4.46023100 & -2.17670500 \\
\hline $\mathrm{H}$ & 4.60501000 & 3.43709600 & -1.69761300 \\
\hline $\mathrm{H}$ & 0.79728300 & 5.17693000 & -2.66980200 \\
\hline $\mathrm{H}$ & 3.23793600 & 5.43872200 & -2.26564600 \\
\hline $\mathrm{C}$ & 0.63257200 & -3.00944400 & -0.94328000 \\
\hline
\end{tabular}




\begin{tabular}{|c|c|c|c|}
\hline $\mathrm{C}$ & -0.44485900 & -3.89297500 & -0.78564700 \\
\hline $\mathrm{C}$ & 1.93066800 & -3.51735000 & -0.80301600 \\
\hline 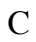 & -0.22544800 & -5.23149300 & -0.48472900 \\
\hline $\mathrm{H}$ & -1.46195800 & -3.50883200 & -0.86858300 \\
\hline $\mathrm{C}$ & 2.14700100 & -4.85624900 & -0.49539800 \\
\hline $\mathrm{H}$ & 2.78474400 & -2.86155500 & -0.96887900 \\
\hline $\mathrm{C}$ & 1.06969200 & -5.72015000 & -0.33264500 \\
\hline $\mathrm{H}$ & -1.07774000 & -5.89814900 & -0.35955000 \\
\hline $\mathrm{H}$ & 3.16634200 & -5.22683900 & -0.39434000 \\
\hline $\mathrm{H}$ & 1.23734800 & -6.76886800 & -0.09219000 \\
\hline $\mathrm{O}$ & 8.54750000 & -0.87434100 & 1.07514400 \\
\hline $\mathrm{C}$ & 9.60340600 & -0.07043300 & 0.61349300 \\
\hline $\mathrm{H}$ & 10.49643300 & -0.70059000 & 0.61822900 \\
\hline $\mathrm{H}$ & 9.42188600 & 0.28856700 & -0.41113200 \\
\hline $\mathrm{H}$ & 9.77082200 & 0.79605600 & 1.27151900 \\
\hline $\mathrm{C}$ & -1.93405100 & 1.68772300 & 1.69864300 \\
\hline $\mathrm{C}$ & -2.28524900 & 2.88486100 & 2.32977600 \\
\hline $\mathrm{C}$ & -2.59431300 & 3.99864300 & 1.57135800 \\
\hline $\mathrm{H}$ & -2.32044200 & 2.92507500 & 3.41581900 \\
\hline $\mathrm{C}$ & -2.21601200 & 2.69676200 & -0.38795800 \\
\hline $\mathrm{C}$ & -2.57643600 & 3.90614400 & 0.17909800 \\
\hline $\mathrm{H}$ & -2.86243800 & 4.93486400 & 2.05837300 \\
\hline $\mathrm{H}$ & -2.20692600 & 2.54623400 & -1.46650500 \\
\hline $\mathrm{H}$ & -2.83595900 & 4.75012400 & -0.45468600 \\
\hline $\mathrm{N}$ & -1.87595100 & 1.63145100 & 0.34656400 \\
\hline $\mathrm{O}$ & 1.19702500 & 1.41335500 & 1.16161800 \\
\hline $\mathrm{C}$ & -1.28999700 & -0.63421200 & 1.48673200 \\
\hline $\mathrm{H}$ & -4.48825900 & -1.28853500 & 2.32550900 \\
\hline $\mathrm{C}$ & -5.59559800 & 1.82056200 & 1.59584100 \\
\hline $\mathrm{C}$ & -6.74391900 & 0.92799500 & 1.11031300 \\
\hline $\mathrm{H}$ & -5.92621800 & 2.63632800 & 2.25071400 \\
\hline $\mathrm{H}$ & -5.02315200 & 2.24918400 & 0.76021000 \\
\hline $\mathrm{H}$ & -7.65644300 & 1.04333300 & 1.71230000 \\
\hline $\mathrm{H}$ & -7.00099600 & 1.10555700 & 0.05785900 \\
\hline $\mathrm{C}$ & -4.63129200 & -2.26235200 & -1.02618900 \\
\hline $\mathrm{C}$ & -5.02760200 & -0.86107100 & -1.46857900 \\
\hline $\mathrm{H}$ & -4.80970300 & -3.02817300 & -1.79131300 \\
\hline $\mathrm{H}$ & -5.14462800 & -2.55741300 & -0.09924100 \\
\hline $\mathrm{H}$ & -4.96112400 & -0.74124200 & -2.56110500 \\
\hline $\mathrm{H}$ & -6.02948100 & -0.57930900 & -1.12749700 \\
\hline B & -5.10022100 & -0.32929500 & 1.98820500 \\
\hline B & -2.92717200 & -0.81185100 & -0.65191200 \\
\hline $\mathrm{O}$ & -4.74417400 & 0.94259400 & 2.32193800 \\
\hline $\mathrm{O}$ & -6.25974200 & -0.40267900 & 1.26414700 \\
\hline , & -3.23240600 & -2.15474300 & -0.77473100 \\
\hline
\end{tabular}

$\mathrm{H}$

$\mathrm{H}$

$\mathrm{H}$

$\mathrm{H}$

$\mathrm{H}$

$\mathrm{H}$

C

$\mathrm{H}$

C

$\mathrm{N}$

$\mathrm{H}$

C

C

C

$\mathrm{H}$

$\mathrm{H}$

C

$\mathrm{H}$

$\mathrm{H}$

O

C

C

C

$\mathrm{H}$

$\mathrm{H}$

C

C

$\mathrm{C}$

C

$\mathrm{H}$

C

$\mathrm{H}$

$\mathrm{H}$

$\mathrm{H}$

$\mathrm{O}$

C

Sum of electronic and zero-point Energies = $-2456.107866$

Sum of electronic and thermal Free Energies = $-2456.193194$

$\begin{array}{crrr}\text { Rh } & -0.76405100 & 0.25746400 & 0.08250700 \\ \mathrm{C} & -1.49410100 & -0.92613000 & 2.61621900 \\ \mathrm{C} & -0.33028700 & -2.53573000 & 1.23225800 \\ \mathrm{C} & 1.15841400 & 0.19729800 & -1.25435800 \\ \mathrm{C} & 0.10076300 & -0.58847800 & -1.83637600 \\ \mathrm{C} & -0.89508500 & 0.36756300 & -2.25009100 \\ \mathrm{C} & -0.47018000 & 1.66639600 & -1.91285800 \\ \mathrm{C} & 0.82403000 & 1.60817400 & -1.35760900 \\ \mathrm{C} & 2.29938100 & -0.31549200 & -0.43184200 \\ \mathrm{C} & -1.50086700 & -1.84027800 & 3.67253900 \\ \mathrm{C} & -0.92443900 & -3.09507400 & 3.51258400 \\ \mathrm{C} & -0.34878800 & -3.44440000 & 2.29322400 \\ \mathrm{C} & -2.14864400 & 0.48343400 & -3.05145900 \\ \mathrm{H} & 0.10773800 & -2.83526700 & 0.27785700\end{array}$

$\begin{array}{lll}-1.95288000 & -1.57526800 & 4.62800900\end{array}$

$\begin{array}{lll}0.09070600 & -4.43232300 & 2.16265800\end{array}$

$\begin{array}{lll}-0.92929900 & -3.80344800 & 4.33840300\end{array}$

$\begin{array}{llll}2.18529400 & -1.39904900 & -0.27641800\end{array}$

$\begin{array}{rrr}-1.92258400 & 0.33668500 & -4.12416400\end{array}$

$\begin{array}{lll}3.27840700 & -0.18605400 & -0.91393400\end{array}$

$\begin{array}{llll}-2.97434300 & -0.18593500 & -2.77978000\end{array}$

$\begin{array}{lll}-1.46311900 & 2.63693300 & -2.46193200\end{array}$

$\begin{array}{lll}-1.06000100 & 3.15439600 & -3.35203400\end{array}$

$\begin{array}{llll}-1.80912900 & 3.41064700 & -1.76152900\end{array}$

$\begin{array}{lll}2.27888700 & 0.34363000 & 0.93808800\end{array}$

$\begin{array}{lll}3.43615800 & 0.40823100 & 1.62515200\end{array}$

$\begin{array}{lll}3.35374000 & 0.86218800 & 2.53132200\end{array}$

$\begin{array}{lll}4.71256900 & -0.07163600 & 1.19744800\end{array}$

$\begin{array}{llll}5.69877400 & 0.83041500 & 0.81466800\end{array}$

$\begin{array}{llll}4.98406200 & -1.44236400 & 1.18326100\end{array}$

$\begin{array}{llll}6.95356100 & 0.38076700 & 0.41541900\end{array}$

$\begin{array}{llll}5.47690900 & 1.89735300 & 0.83262400\end{array}$

$\begin{array}{llll}6.22219200 & -1.89966300 & 0.77334600\end{array}$

$\begin{array}{lll}4.21607700 & -2.14378600 & 1.50886100\end{array}$

$\begin{array}{lll}7.21872000 & -0.99168000 & 0.38977200\end{array}$

$\begin{array}{lll}7.71304900 & 1.10281200 & 0.12765600\end{array}$

$\begin{array}{rrr}6.45942400 & -2.96096300 & 0.75392100\end{array}$

$\begin{array}{llll}-2.57570900 & 1.82041300 & -2.79848400\end{array}$

$\begin{array}{llll}1.64995400 & 2.73910100 & -0.94380900\end{array}$

$\begin{array}{llll}3.03058200 & 2.74324200 & -1.18069600\end{array}$

$\begin{array}{llll}1.06695100 & 3.85908900 & -0.33644000\end{array}$

$\begin{array}{llll}3.80492200 & 3.83907300 & -0.81810800\end{array}$

$\begin{array}{llll}3.49549500 & 1.90071100 & -1.69236400\end{array}$

$\begin{array}{llll}1.84254800 & 4.94743900 & 0.03420700\end{array}$

$\begin{array}{llll}-0.00277600 & 3.85438300 & -0.12562400\end{array}$

$\begin{array}{llll}3.21518500 & 4.94095200 & -0.20625200\end{array}$

$\begin{array}{llll}4.87284500 & 3.83789500 & -1.03083900\end{array}$

$\begin{array}{llll}1.37726800 & 5.80552300 & 0.51512700\end{array}$

$\begin{array}{rrrr}3.82226500 & 5.79798000 & 0.07804400\end{array}$

$\begin{array}{llll}0.11194400 & -2.02151900 & -2.15879500\end{array}$

$\begin{array}{lll}-1.08540700 & -2.74788000 & -2.20054800\end{array}$

$\begin{array}{lll}1.31119500 & -2.67714200 & -2.46124300\end{array}$

$\begin{array}{llll}-1.07589700 & -4.09983800 & -2.51438100\end{array}$

$\begin{array}{llll}-2.03007700 & -2.26436600 & -1.94675900\end{array}$

$\begin{array}{lll}1.31733600 & -4.03412400 & -2.76723400\end{array}$

$\begin{array}{llll}2.24452500 & -2.11539900 & -2.49127900\end{array}$

$\begin{array}{llll}0.12519300 & -4.75005100 & -2.79044000\end{array}$

$\begin{array}{lll}-2.01603500 & -4.64903700 & -2.53868400\end{array}$

$\begin{array}{llll}2.25745200 & -4.52896300 & -3.00310500\end{array}$

$\begin{array}{llll}0.13000500 & -5.81087400 & -3.03276500\end{array}$

$\begin{array}{llll}8.39024600 & -1.53722600 & 0.01697800\end{array}$

$\begin{array}{llll}9.44441200 & -0.67587500 & -0.35431900\end{array}$

$\begin{array}{llll}10.29054200 & -1.31939300 & -0.60408100\end{array}$

$\begin{array}{llll}9.17794400 & -0.07174800 & -1.23372500\end{array}$

$\begin{array}{llll}9.72810900 & -0.01033200 & 0.47356900\end{array}$

$\begin{array}{lll}-2.08485800 & 0.40670500 & 2.68741800\end{array}$

$\begin{array}{lll}-2.82696800 & 0.92321300 & 3.74676500\end{array}$

$\begin{array}{lll}-3.32957300 & 2.21286900 & 3.66666200\end{array}$

$\begin{array}{lll}-3.01554300 & 0.31100100 & 4.62480000\end{array}$

$\begin{array}{lll}-2.36586600 & 2.40218200 & 1.49967000\end{array}$

$\begin{array}{lll}-3.09314500 & 2.97548300 & 2.52704700\end{array}$

$\begin{array}{lll}-3.91304000 & 2.62054600 & 4.48934100\end{array}$

$\begin{array}{lll}-2.17708700 & 2.92889500 & 0.56429800\end{array}$

$\begin{array}{lll}-3.48382000 & 3.98365800 & 2.42231500\end{array}$

$\begin{array}{lll}-1.87110500 & 1.16454100 & 1.58624800\end{array}$

$\begin{array}{lll}1.22722900 & 0.78985700 & 1.41205600\end{array}$

$\begin{array}{lll}-0.88110000 & -1.27130100 & 1.39767800\end{array}$

$\begin{array}{lll}-2.11685900 & -0.39711300 & -0.33052000\end{array}$

$\begin{array}{lll}-4.21522000 & -2.95754800 & 1.54305600\end{array}$

$\begin{array}{lll}-3.72795900 & -3.41004200 & 0.16068200\end{array}$ 


$\begin{array}{lrrr}\mathrm{H} & -3.47451800 & -3.13345700 & 2.33262600 \\ \mathrm{H} & -5.15945800 & -3.43522500 & 1.83625300 \\ \mathrm{H} & -2.64550200 & -3.60907600 & 0.14615300 \\ \mathrm{H} & -4.25171400 & -4.29698000 & -0.21522600 \\ \mathrm{C} & -5.55258100 & 1.71442600 & -2.14240000 \\ \mathrm{C} & -5.14473500 & 2.47090700 & -0.87165500 \\ \mathrm{H} & -5.04557700 & 2.08870200 & -3.03733000 \\ \mathrm{H} & -6.63711500 & 1.72596700 & -2.31211400 \\ \mathrm{H} & -4.26627300 & 3.11269100 & -1.03960600 \\ \mathrm{H} & -5.95028000 & 3.08790900 & -0.45618600 \\ \mathrm{~B} & -4.37749300 & -1.24215200 & 0.08255000 \\ \mathrm{~B} & -4.77444900 & 0.26021300 & -0.59600300 \\ \mathrm{O} & -4.43909200 & -1.55282500 & 1.41166500 \\ \mathrm{O} & -4.00065500 & -2.30447500 & -0.70190300 \\ \mathrm{O} & -5.14668900 & 0.36562900 & -1.90597400 \\ \mathrm{O} & -4.79704900 & 1.45615800 & 0.06989800\end{array}$

\section{TS2-Rh(IV)}

Sum of electronic and zero-point Energies = $-2456.061044$

Sum of electronic and thermal Free Energies = $-2456.146396$

$\begin{array}{crrr}\text { Rh } & -1.07356700 & 0.38453500 & -0.23887600 \\ \mathrm{C} & -1.40174000 & -0.26917400 & 2.60184000 \\ \mathrm{C} & -0.66587000 & -2.22496300 & 1.39914100 \\ \mathrm{C} & 1.01018200 & 0.22366600 & -1.04731800 \\ \mathrm{C} & 0.14959000 & -0.67406400 & -1.79320500 \\ \mathrm{C} & -0.74737000 & 0.16737100 & -2.51764000 \\ \mathrm{C} & -0.49346600 & 1.51938400 & -2.21182100 \\ \mathrm{C} & 0.64980300 & 1.59687200 & -1.37380400 \\ \mathrm{C} & 2.09897800 & -0.20752300 & -0.11932000 \\ \mathrm{C} & -1.37684200 & -0.99358800 & 3.79546400 \\ \mathrm{C} & -0.99627800 & -2.33096000 & 3.79476100 \\ \mathrm{C} & -0.64486800 & -2.94615200 & 2.59573300 \\ \mathrm{C} & -1.68014300 & 0.13069000 & -3.67609300 \\ \mathrm{H} & -0.40189000 & -2.73820500 & 0.47207400 \\ \mathrm{H} & -1.65600300 & -0.51998800 & 4.73657600 \\ \mathrm{H} & -0.34514000 & -3.99402100 & 2.58835500 \\ \mathrm{H} & -0.97252800 & -2.89023400 & 4.72827600 \\ \mathrm{H} & 1.90967500 & -1.24860200 & 0.18519500 \\ \mathrm{H} & -1.09848400 & -0.03358700 & -4.60295100 \\ \mathrm{H} & 3.06945100 & -0.23266000 & -0.63910400 \\ \mathrm{H} & -2.47681800 & -0.61657000 & -3.63485600 \\ \mathrm{C} & -1.34069900 & 2.36122000 & -3.11351700 \\ \mathrm{H} & -0.73355800 & 2.83281600 & -3.90782600 \\ \mathrm{H} & -1.92102000 & 3.15484200 & -2.62335100 \\ \mathrm{C} & 2.21326100 & 0.61663100 & 1.15811100 \\ \mathrm{~N} & 3.44949900 & 0.63346200 & 1.73845500 \\ \mathrm{H} & 3.48856200 & 1.20111800 & 2.57992900 \\ \mathrm{C} & 4.63037600 & -0.02559000 & 1.28993500 \\ \mathrm{C} & 5.68818100 & 0.71290200 & 0.76920400 \\ \mathrm{C} & 4.74774900 & -1.41638100 & 1.37998500 \\ \mathrm{C} & 6.85402400 & 0.08611000 & 0.33787600 \\ \mathrm{H} & 5.59702900 & 1.79741600 & 0.70709900 \\ \mathrm{C} & 5.89250900 & -2.05162000 & 0.93652400 \\ \mathrm{H} & 3.92969800 & -1.99241000 & 1.81205500 \\ \mathrm{C} & 6.95710400 & -1.30498800 & 0.41479500 \\ \mathrm{H} & 7.66855700 & 0.68708400 & -0.05780100 \\ \mathrm{H} & 6.00421800 & -3.13202500 & 0.99729700 \\ \mathrm{O} & -2.26779300 & 1.42869900 & -3.66915800 \\ \mathrm{C} & 1.32992100 & 2.82424400 & -0.97032500 \\ \mathrm{C} & 2.72606500 & 2.87286200 & -0.86112100 \\ \mathrm{C} & 0.60091000 & 4.00202200 & -0.76316400 \\ \mathrm{C} & 3.36783400 & 4.06077900 & -0.53729000 \\ & 3.31975200 & 1.98047500 & -1.06023600 \\ & 1.24223500 & 5.18619000 & -0.43057700\end{array}$

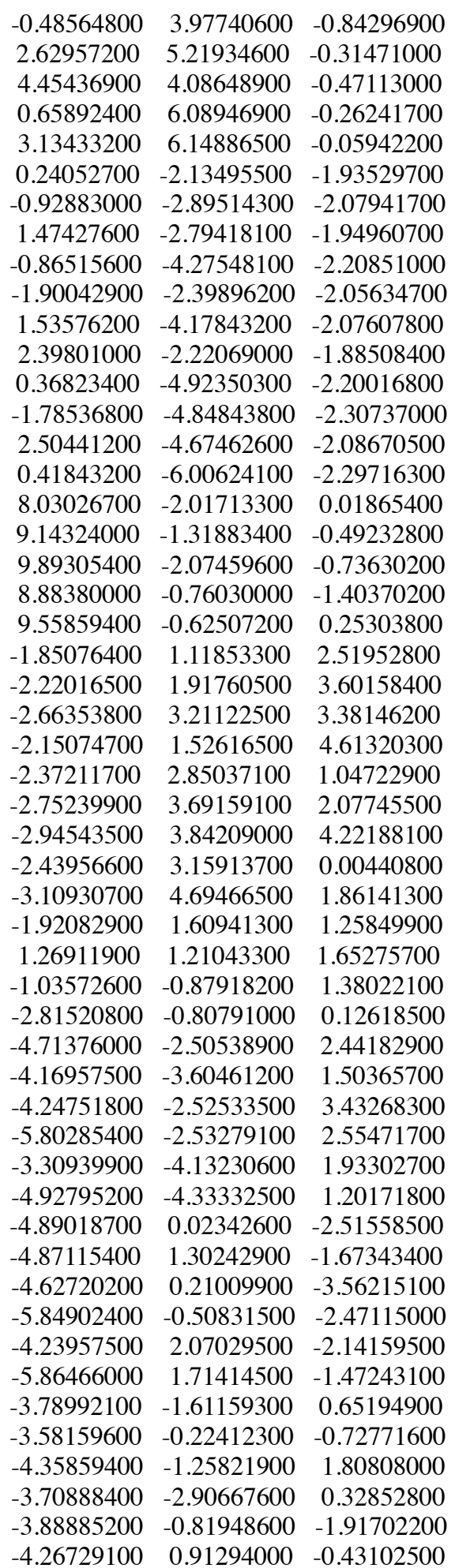

\section{INT4-Rh(IV)}

Sum of electronic and zero-point Energies = $-2456.158644$

Sum of electronic and thermal Free Energies = $-2456.246168$

$\begin{array}{crrr}\text { Rh } & 0.61947700 & 0.26399600 & 0.34333400 \\ \mathrm{C} & 1.98381000 & -0.57056000 & -1.99852200 \\ \mathrm{C} & 1.09105400 & -2.62170700 & -1.07593300 \\ \mathrm{C} & -1.51668800 & 0.75177400 & 1.03350100 \\ \mathrm{C} & -1.10369000 & -0.51698000 & 1.60405600 \\ \mathrm{C} & -0.01251700 & -0.23131000 & 2.49420100 \\ \mathrm{C} & 0.34150300 & 1.11576600 & 2.37210800 \\ \mathrm{C} & -0.55332700 & 1.75083500 & 1.42575800\end{array}$




$\begin{array}{lrrr}\mathrm{N} & 2.07976600 & 1.40340500 & -0.70106500 \\ \mathrm{O} & -1.20042600 & 0.43095000 & -1.80265000 \\ \mathrm{C} & 1.80532300 & -1.42217600 & -0.87138300 \\ \mathrm{H} & 4.36781500 & -2.98463000 & -1.36504800 \\ \mathrm{C} & 6.31365000 & -0.24454100 & -1.69127200 \\ \mathrm{C} & 7.13964500 & -1.14325700 & -0.76726600 \\ \mathrm{H} & 6.88426200 & 0.14892900 & -2.54056800 \\ \mathrm{H} & 5.85790000 & 0.59637400 & -1.14583800 \\ \mathrm{H} & 8.02539500 & -1.56048100 & -1.26499400 \\ \mathrm{H} & 7.46697300 & -0.63520700 & 0.14778700 \\ \mathrm{C} & 3.82753500 & -2.23886600 & 2.16562100 \\ \mathrm{C} & 4.42713100 & -0.88554700 & 1.79717400 \\ \mathrm{H} & 3.59947900 & -2.33052200 & 3.23393700 \\ \mathrm{H} & 4.47448600 & -3.07327800 & 1.86168900 \\ \mathrm{H} & 4.13355400 & -0.10387700 & 2.51145500 \\ \mathrm{H} & 5.51750900 & -0.91652300 & 1.70028300 \\ \mathrm{~B} & 5.21981000 & -2.15732000 & -1.31763500 \\ \mathrm{~B} & 2.72675800 & -1.36752600 & 0.40586900 \\ \mathrm{O} & 5.26588000 & -1.08838400 & -2.16664100 \\ \mathrm{O} & 6.25669200 & -2.21415600 & -0.43340000 \\ \mathrm{O} & 2.61591800 & -2.30511300 & 1.40509000 \\ \mathrm{O} & 3.84778500 & -0.58248200 & 0.52440800\end{array}$

\section{INT3-Rh(II)}

Sum of electronic and zero-point Energies = -2456.333289

Sum of electronic and thermal Free Energies = $-2456.420782$

$\begin{array}{crrr}\mathrm{Rh} & 1.14083200 & -0.27821000 & -0.12533800 \\ \mathrm{C} & 1.84500200 & 0.27635300 & 2.66968600 \\ \mathrm{C} & 0.74514200 & 2.15956400 & 1.65506700 \\ \mathrm{C} & -1.23699700 & -0.00478400 & -1.04477100 \\ \mathrm{C} & -0.21390700 & 0.77185700 & -1.70407200 \\ \mathrm{C} & 0.65961600 & -0.17989300 & -2.32088600 \\ \mathrm{C} & 0.20617000 & -1.47318400 & -2.02598300 \\ \mathrm{C} & -1.03832900 & -1.39142700 & -1.31839800 \\ \mathrm{C} & -2.26248300 & 0.57608900 & -0.12019800 \\ \mathrm{C} & 1.86381700 & 0.95093900 & 3.89541900 \\ \mathrm{C} & 1.32457100 & 2.22620500 & 4.00666100 \\ \mathrm{C} & 0.76617200 & 2.82906500 & 2.87635700 \\ \mathrm{C} & 1.68673400 & -0.26105900 & -3.39864700 \\ \mathrm{H} & 0.31679100 & 2.66344100 & 0.78309500 \\ \mathrm{H} & 2.28011400 & 0.46365700 & 4.77993500 \\ \mathrm{H} & 0.34519000 & 3.83396700 & 2.94945500 \\ \mathrm{H} & 1.32844200 & 2.74382100 & 4.96618100 \\ \mathrm{H} & -1.94547400 & 1.59566100 & 0.15830000 \\ \mathrm{H} & 1.29062200 & 0.12639600 & -4.35814600 \\ \mathrm{H} & -3.24267100 & 0.69430100 & -0.60836000 \\ \mathrm{H} & 2.63754000 & 0.24622000 & -3.18557600 \\ \mathrm{C} & 0.93226500 & -2.43586200 & -2.90379700 \\ \mathrm{H} & 0.26345200 & -2.87474900 & -3.67255900 \\ \mathrm{H} & 1.41827400 & -3.27904600 & -2.38366400 \\ \mathrm{C} & -2.43096900 & -0.17578100 & 1.18904500 \\ \mathrm{~N} & -3.70156000 & -0.16448600 & 1.76664200 \\ \mathrm{H} & -3.73593700 & -0.81681000 & 2.54357500 \\ \mathrm{C} & -4.93776900 & 0.25643200 & 1.24118600 \\ \mathrm{C} & -6.00441200 & -0.63859100 & 1.18012000 \\ \mathrm{C} & -5.15049900 & 1.57638500 & 0.82294900 \\ \mathrm{C} & -7.25580600 & -0.24444000 & 0.71157100 \\ \mathrm{H} & -5.84227600 & -1.67002800 & 1.49453500 \\ \mathrm{C} & -6.38211400 & 1.96953100 & 0.32899000 \\ \mathrm{H} & -4.33649900 & 2.29580100 & 0.89836700 \\ \mathrm{C} & -7.44499200 & 1.06442000 & 0.27260600 \\ \mathrm{H} & -8.06342200 & -0.97206400 & 0.68050700 \\ \mathrm{H} & -6.55486500 & 2.99033500 & -0.00663300 \\ \mathrm{O} & 1.96148300 & -1.66331800 & -3.51578000 \\ & & & \end{array}$


$\begin{array}{llll}\text { C } & -1.84869700 & -2.51754300 & -0.90452100\end{array}$

$\begin{array}{lllll}\text { C } & -3.24802200 & -2.42650100 & -0.76742400\end{array}$

$\begin{array}{llll}\text { C } & -1.26384700 & -3.77634800 & -0.66339400\end{array}$

C $\quad-4.01156700 \quad-3.52464100 \quad-0.39615400$

$\mathrm{H} \quad-3.74757800 \quad-1.48458400 \quad-0.99702700$

C $\quad-2.02933800 \quad-4.87330800 \quad-0.29894000$

$\mathrm{H} \quad-0.18008900 \quad-3.86981100 \quad-0.73986100$

C $\quad-3.41255400 \quad-4.76061300 \quad-0.15733800$

$\mathrm{H} \quad-5.09509300 \quad-3.41708500 \quad-0.31701800$

$\mathrm{H} \quad-1.53913600 \quad-5.82909800 \quad-0.11080800$

$\mathrm{H} \quad-4.01297200 \quad-5.62291900 \quad 0.13063600$

$\begin{array}{lllll}\mathrm{C} & -0.11760600 & 2.22709600 & -1.84190500\end{array}$

$\begin{array}{lllll}\text { C } & 1.14093300 & 2.85495100 & -1.88626800\end{array}$

C $\quad-1.25608100 \quad 3.03966600 \quad-1.94844800$

C $\quad 1.24686300 \quad 4.23187100 \quad-2.01752000$

$\begin{array}{llll}\mathrm{H} & 2.04209900 & 2.25338700 & -1.75063900\end{array}$

C $\quad-1.14672500 \quad 4.42281800 \quad-2.06374200$

$\begin{array}{lllll}\mathrm{H} & -2.24218600 & 2.57443100 & -1.96341900\end{array}$

$\begin{array}{llll}\mathrm{C} & 0.10456500 & 5.02886500 & -2.09904900\end{array}$

$\mathrm{H} \quad 2.23873000 \quad 4.68477400 \quad-2.03538300$

$\mathrm{H} \quad-2.05044000 \quad 5.02866300 \quad-2.13885000$

$\mathrm{H} \quad \begin{array}{llll}0.19086300 & 6.11162100 & -2.18838800\end{array}$

$\begin{array}{lllll}\mathrm{O} & -8.61842600 & 1.55480500 & -0.21638200\end{array}$

$\begin{array}{lllll}\text { C } & -9.69797700 & 0.66754500 & -0.31311600\end{array}$

$\mathrm{H} \quad-10.53211000 \quad 1.23577400 \quad-0.73503100$

$\mathrm{H} \quad-9.47054600 \quad-0.18198000 \quad-0.97654600$

$\begin{array}{llll}\mathrm{H} & -9.99479900 & 0.27440400 & 0.67259600\end{array}$

$\begin{array}{llll}\text { C } & 2.39252700 & -1.06384100 & 2.49354700\end{array}$

$\begin{array}{llll}\text { C } & 3.32607700 & -1.66586400 & 3.34127600\end{array}$

$\begin{array}{llll}\text { C } & 3.79863100 & -2.93704000 & 3.05680900\end{array}$

$\mathrm{H} \quad 3.69890000 \quad-1.11543400 \quad 4.20357900$

$\begin{array}{llll}\text { C } & 2.42837000 & -2.92379700 & 1.10705000\end{array}$

$\begin{array}{llll}\text { C } & 3.33480500 & -3.58973900 & 1.91466800\end{array}$

$\begin{array}{llll}\mathrm{H} & 4.53345400 & -3.40968600 & 3.70772200\end{array}$

$\begin{array}{llll}\mathrm{H} & 2.04451400 & -3.38163200 & 0.19218000\end{array}$

$\mathrm{H} \quad 3.67302700 \quad-4.59023600 \quad 1.65095900$

$\begin{array}{llll}\mathrm{N} & 1.95897000 & -1.70174000 & 1.37644700\end{array}$

$\begin{array}{llll}\mathrm{O} & -1.53589400 & -0.74787800 & 1.77228900\end{array}$

$\begin{array}{llll}\mathrm{C} & 1.27178600 & 0.86575300 & 1.51574300\end{array}$

$\begin{array}{llll}\mathrm{H} & 2.64582400 & 0.27438100 & -0.35847600\end{array}$

$\begin{array}{llll}\mathrm{C} & 4.37886000 & 2.78181000 & 1.90285400\end{array}$

$\begin{array}{llll}\text { C } & 3.88446200 & 3.49447400 & 0.63645800\end{array}$

$\begin{array}{llll}\mathrm{H} & 3.62299100 & 2.76878400 & 2.69982300\end{array}$

$\begin{array}{llll}\mathrm{H} & 5.30248900 & 3.23219100 & 2.30229900\end{array}$

$\begin{array}{llll}\mathrm{H} & 2.79154500 & 3.63093200 & 0.64938800\end{array}$

$\begin{array}{llll}\mathrm{H} & 4.35720300 & 4.47528000 & 0.48016600\end{array}$

$\begin{array}{llll}\text { C } & 4.99667900 & -1.31184300 & -2.62024800\end{array}$

C $\quad 4.70756900-2.15562400 \quad-1.38401200$

$\mathrm{H} \quad 4.33053000 \quad-1.55751300 \quad-3.45586800$

$\mathrm{H} \quad 6.04619700 \quad-1.39734600 \quad-2.94982600$

$\mathrm{H} \quad 3.65306000 \quad-2.48064900 \quad-1.38581200$

$\mathrm{H} \quad 5.35403000 \quad-3.03791600 \quad-1.28145800$

$\begin{array}{llll}\text { B } & 4.52839200 & 1.39386700 & 0.12256200\end{array}$

$\begin{array}{lllll}\text { B } & 4.71873100 & 0.00101800 & -0.79890000\end{array}$

$\begin{array}{llll}\mathrm{O} & 4.65677700 & 1.45264400 & 1.49117000\end{array}$

$\begin{array}{llll}\mathrm{O} & 4.22748900 & 2.62540700 & -0.43099100\end{array}$

$\begin{array}{lllll}\mathrm{O} & 4.75601500 & 0.01986800 & -2.18491700\end{array}$

O $\quad 4.91502600 \quad-1.27162700 \quad-0.29527300$

\section{TS2A-Rh(II)}

Sum of electronic and zero-point Energies = $-2456.306208$

Sum of electronic and thermal Free Energies = $-2456.393419$

$\begin{array}{llll}\mathrm{Rh} & -0.72497100 & 0.43958600 & 0.32575500\end{array}$

$\begin{array}{llll}\text { C } & -2.01064200 & -0.28112400 & 2.85210700\end{array}$
$-0.14379600 \quad-1.73476400 \quad 2.43210600$

$\begin{array}{lll}1.30553500 & 0.10313800 & -0.53022500\end{array}$

$\begin{array}{lll}0.33012500 & -0.67924400 & -1.27060400\end{array}$

$-0.51103500 \quad 0.26913800-1.93363700$

$\begin{array}{llll}-0.09153500 & 1.56733400 & -1.60289400\end{array}$

$\begin{array}{lll}1.07723100 & 1.50675400 & -0.79376900\end{array}$

$\begin{array}{lll}2.47692200 & -0.47969700 & 0.19498800\end{array}$

$\begin{array}{lll}-2.27583500 & -0.97069000 & 4.03950200\end{array}$

$\begin{array}{lll}-1.47704900 & -2.03922900 & 4.42328800\end{array}$

$\begin{array}{lll}-0.41237000 & -2.42563200 & 3.60957500\end{array}$

$\begin{array}{lll}-1.63177500 & 0.36103000 & -2.90952700\end{array}$

$\begin{array}{llll}0.68201000 & -2.07550900 & 1.80338500\end{array}$

$\begin{array}{lll}-3.12658100 & -0.68854900 & 4.66245700\end{array}$

$\begin{array}{lll}0.20821500 & -3.27653500 & 3.89497700\end{array}$

$\begin{array}{lll}-1.69268100 & -2.57966600 & 5.34471800\end{array}$

$\begin{array}{lll}2.22247700 & -1.50549300 & 0.50803300\end{array}$

$\begin{array}{lll}-1.29170800 & 0.10096000 & -3.93098200\end{array}$

$\begin{array}{lll}3.32757200 & -0.59204300 & -0.49548000\end{array}$

$\begin{array}{lll}-2.51885000 & -0.24006500 & -2.67606900\end{array}$

$\begin{array}{lll}-0.93569300 & 2.53808200 & -2.35802000\end{array}$

$\begin{array}{lll}-0.37263900 & 3.01268200 & -3.18620000\end{array}$

$\begin{array}{lll}-1.36940400 & 3.34770500 & -1.75013400\end{array}$

$\begin{array}{lll}2.92909500 & 0.26074000 & 1.43822500\end{array}$

$\begin{array}{lll}4.29676600 & 0.24271600 & 1.70785700\end{array}$

$\begin{array}{lll}4.51433700 & 0.87532500 & 2.47123200\end{array}$

$\begin{array}{lll}5.37986100 & -0.22897300 & 0.94092600\end{array}$

$\begin{array}{lll}6.44646500 & 0.62219200 & 0.65877700\end{array}$

$\begin{array}{llll}5.44790400 & -1.55482200 & 0.49242800\end{array}$

$\begin{array}{llll}7.55846600 & 0.17956300 & -0.05410800\end{array}$

$\begin{array}{llll}6.39734300 & 1.65669900 & 1.00011200\end{array}$

$\begin{array}{lll}6.53439200 & -1.99484300 & -0.24265200\end{array}$

$\begin{array}{lll}4.63986000 & -2.24372400 & 0.73440400\end{array}$

$\begin{array}{lll}7.60011800 & -1.13386800 & -0.51822800\end{array}$

$\begin{array}{lll}8.37275400 & 0.87271900 & -0.25144700\end{array}$

$\begin{array}{lll}6.59218400 & -3.01998900 & -0.60314100\end{array}$

$\begin{array}{lll}-2.00687800 & 1.74725700 & -2.86203500\end{array}$

$\begin{array}{lll}1.86747500 & 2.65166500 & -0.34076400\end{array}$

$\begin{array}{lll}3.26251000 & 2.66648800 & -0.47183000\end{array}$

$\begin{array}{lll}1.24079600 & 3.78485300 & 0.19573000\end{array}$

$\begin{array}{llll}4.00652700 & 3.76884800 & -0.06524400\end{array}$

$\begin{array}{lll}3.76753100 & 1.80835900 & -0.91860300\end{array}$

$\begin{array}{llll}1.98294700 & 4.88843300 & 0.59214500\end{array}$

$\begin{array}{lll}0.15887800 & 3.76350600 & 0.33605900\end{array}$

$\begin{array}{llll}3.37139300 & 4.88635500 & 0.46772300\end{array}$

$\begin{array}{llll}5.09079800 & 3.75968500 & -0.18415300\end{array}$

$\begin{array}{lll}1.47527300 & 5.75356700 & 1.01775400\end{array}$

$\begin{array}{lll}3.95292100 & 5.75115100 & 0.78546500\end{array}$

$\begin{array}{llll}0.21391000 & -2.14356800 & -1.38220700\end{array}$

$-1.05254800-2.74467600-1.32029500$

$\begin{array}{lll}1.33416100 & -2.95817400 & -1.58668300\end{array}$

$\begin{array}{lll}-1.18809800 & -4.11852500 & -1.46070200\end{array}$

$-1.94211400-2.13909100-1.12418800$

$\begin{array}{lll}1.19548900 & -4.33788300 & -1.71510400\end{array}$

$\begin{array}{llll}2.32333600 & -2.50483000 & -1.67152900\end{array}$

$-0.06568100-4.92228900-1.65388200$

$\begin{array}{llll}-2.19037100 & -4.54193100 & -1.40380900\end{array}$

$\begin{array}{llll}2.07934900 & -4.95631900 & -1.87407800\end{array}$

$-0.17282200 \quad-6.00232200-1.75496700$

$\begin{array}{llll}8.62620200 & -1.66904400 & -1.23412300\end{array}$

$\begin{array}{llll}9.70399100 & -0.82994700 & -1.54815000\end{array}$

$10.40872100 \quad-1.43220900 \quad-2.12847600$

$\begin{array}{llll}9.38610300 & 0.03328100 & -2.15392800\end{array}$

$\begin{array}{lll}10.21042800 & -0.45869300 & -0.64286100\end{array}$

$\begin{array}{lll}-2.87925000 & 0.77445300 & 2.33617400\end{array}$

$\begin{array}{lll}-4.03686200 & 1.25381700 & 2.95060100\end{array}$

$\begin{array}{lll}-4.83707600 & 2.16635100 & 2.28735900\end{array}$ 


$\begin{array}{lrrr}\mathrm{H} & -4.32698300 & 0.87204900 & 3.92731100 \\ \mathrm{C} & -3.30247000 & 2.10057400 & 0.46861600 \\ \mathrm{C} & -4.48247400 & 2.58191500 & 1.00739900 \\ \mathrm{H} & -5.75658300 & 2.52370900 & 2.74891800 \\ \mathrm{H} & -2.99081100 & 2.35408400 & -0.54668900 \\ \mathrm{H} & -5.11920100 & 3.23561900 & 0.41733200 \\ \mathrm{~N} & -2.50029500 & 1.26008900 & 1.12978000 \\ \mathrm{O} & 2.19123800 & 0.83594800 & 2.20792200 \\ \mathrm{C} & -0.91873000 & -0.64392300 & 2.02521400 \\ \mathrm{H} & -3.29131600 & -0.67972500 & -0.62690900 \\ \mathrm{C} & -4.89558800 & -2.15995400 & 1.58962200 \\ \mathrm{C} & -4.00408900 & -3.07314600 & 0.75349000 \\ \mathrm{H} & -4.50973900 & -2.02884200 & 2.61945300 \\ \mathrm{H} & -5.91797700 & -2.59042700 & 1.67174600 \\ \mathrm{H} & -2.93954500 & -2.88691800 & 1.02656400 \\ \mathrm{H} & -4.20880900 & -4.14827100 & 0.89979100 \\ \mathrm{C} & -5.04413500 & 0.71034900 & -3.80900000 \\ \mathrm{C} & -5.39222500 & 1.74562300 & -2.73238300 \\ \mathrm{H} & -4.15481500 & 1.00595300 & -4.38758500 \\ \mathrm{H} & -5.87316300 & 0.54502400 & -4.51785300 \\ \mathrm{H} & -4.57058200 & 2.47142700 & -2.60199900 \\ \mathrm{H} & -6.31141100 & 2.30751500 & -2.96190200 \\ \mathrm{~B} & -4.43008000 & -1.23339900 & -0.47731500 \\ \mathrm{~B} & -5.07345100 & -0.29516000 & -1.74329700 \\ \mathrm{O} & -4.90937000 & -0.95515200 & 0.89437300 \\ \mathrm{O} & -4.26757500 & -2.69460600 & -0.56457900 \\ \mathrm{O} & -4.78081300 & -0.48071000 & -3.09944700 \\ \mathrm{O} & -5.55956200 & 1.00200500 & -1.54619000\end{array}$

\begin{tabular}{|c|c|c|c|}
\hline $\mathrm{C}$ & 6.37374300 & -2.41978900 & -0.48838600 \\
\hline $\mathrm{H}$ & 4.48947800 & -2.54136600 & 0.53146900 \\
\hline $\mathrm{C}$ & 7.52076800 & -1.65943700 & -0.73171200 \\
\hline $\mathrm{H}$ & 8.51902900 & 0.22199700 & -0.33157000 \\
\hline $\mathrm{H}$ & 6.30744500 & -3.41121100 & -0.93195000 \\
\hline $\mathrm{O}$ & -1.80866200 & 2.50334700 & -2.37868900 \\
\hline $\mathrm{C}$ & 2.30679500 & 2.70895200 & -0.09823200 \\
\hline $\mathrm{C}$ & 3.68098000 & 2.52963000 & -0.30680700 \\
\hline $\mathrm{C}$ & 1.88145500 & 3.89269000 & 0.52036000 \\
\hline $\mathrm{C}$ & 4.59747000 & 3.49250700 & 0.10086400 \\
\hline $\mathrm{H}$ & 4.03295500 & 1.63247000 & -0.81806300 \\
\hline $\mathrm{C}$ & 2.79593300 & 4.85699700 & 0.92029200 \\
\hline $\mathrm{H}$ & 0.81679600 & 4.02449800 & 0.71704800 \\
\hline $\mathrm{C}$ & 4.16082500 & 4.66179700 & 0.71614500 \\
\hline $\mathrm{H}$ & 5.66089600 & 3.33255700 & -0.08129400 \\
\hline $\mathrm{H}$ & 2.44211300 & 5.76426200 & 1.40905200 \\
\hline $\mathrm{H}$ & 4.87786800 & 5.41726700 & 1.03539600 \\
\hline $\mathrm{C}$ & 0.01981200 & -1.73739000 & -1.41613800 \\
\hline $\mathrm{C}$ & -1.29585200 & -2.22007900 & -1.40519200 \\
\hline $\mathrm{C}$ & 1.05362000 & -2.61052600 & -1.77953600 \\
\hline $\mathrm{C}$ & -1.56793200 & -3.53723300 & -1.75105000 \\
\hline $\mathrm{H}$ & -2.12700400 & -1.57963000 & -1.09486600 \\
\hline $\mathrm{C}$ & 0.78021000 & -3.93399100 & -2.11238300 \\
\hline $\mathrm{H}$ & 2.07899400 & -2.24164200 & -1.83589600 \\
\hline $\mathrm{C}$ & -0.53108000 & -4.39966600 & -2.10070900 \\
\hline $\mathrm{H}$ & -2.61114600 & -3.85170600 & -1.73294300 \\
\hline $\mathrm{H}$ & 1.59723400 & -4.59866700 & -2.39488500 \\
\hline $\mathrm{H}$ & -0.74448100 & -5.43521100 & -2.36613900 \\
\hline $\mathrm{O}$ & 8.46110700 & -2.24062600 & -1.52402300 \\
\hline $\mathrm{C}$ & 9.61825400 & -1.50121300 & -1.80580000 \\
\hline $\mathrm{H}$ & 10.23623800 & -2.12626800 & -2.45649100 \\
\hline $\mathrm{H}$ & 9.38382300 & -0.56055300 & -2.32857200 \\
\hline $\mathrm{H}$ & 10.18509900 & -1.26533700 & -0.89105600 \\
\hline $\mathrm{C}$ & -3.07461900 & 0.50147500 & 2.08752000 \\
\hline $\mathrm{C}$ & -4.43946600 & 0.63437300 & 2.33527700 \\
\hline $\mathrm{C}$ & -5.15487700 & 1.63535000 & 1.70455600 \\
\hline $\mathrm{H}$ & -4.95416100 & -0.10770400 & 2.93876200 \\
\hline $\mathrm{C}$ & -3.13155800 & 2.34222900 & 0.66232700 \\
\hline $\mathrm{C}$ & -4.49099800 & 2.51533600 & 0.85649900 \\
\hline $\mathrm{H}$ & -6.23409900 & 1.69302700 & 1.82822600 \\
\hline $\mathrm{H}$ & -2.57730700 & 2.96752200 & -0.03648700 \\
\hline $\mathrm{H}$ & -5.02255000 & 3.29257800 & 0.31279600 \\
\hline $\mathrm{N}$ & -2.43641600 & 1.36859800 & 1.26307400 \\
\hline $\mathrm{O}$ & 2.42727600 & 0.64363800 & 2.31588700 \\
\hline $\mathrm{C}$ & -0.92559200 & -0.64462400 & 2.04208800 \\
\hline $\mathrm{H}$ & -3.74347900 & -0.64540000 & -0.17482600 \\
\hline $\mathrm{C}$ & -5.46112100 & -2.64770100 & 1.25591900 \\
\hline $\mathrm{C}$ & -4.31246800 & -3.25575200 & 0.44026600 \\
\hline $\mathrm{H}$ & -5.19511700 & -2.58322400 & 2.33394900 \\
\hline $\mathrm{H}$ & -6.37030500 & -3.28328100 & 1.19064400 \\
\hline $\mathrm{H}$ & -3.33987300 & -2.98550900 & 0.91405200 \\
\hline $\mathrm{H}$ & -4.35927100 & -4.35777600 & 0.37565000 \\
\hline $\mathrm{C}$ & -4.93042300 & 0.98582700 & -3.67113300 \\
\hline $\mathrm{C}$ & -5.48187800 & 1.89125100 & -2.57254900 \\
\hline $\mathrm{H}$ & -4.02570400 & 1.40035700 & -4.14274900 \\
\hline $\mathrm{H}$ & -5.67272500 & 0.79677300 & -4.46632900 \\
\hline $\mathrm{H}$ & -4.68859900 & 2.55457000 & -2.17803000 \\
\hline $\mathrm{H}$ & -6.31977400 & 2.52128600 & -2.90761500 \\
\hline B & -4.80727000 & -1.28216400 & -0.49573500 \\
\hline B & -5.24165500 & -0.21758600 & -1.74538700 \\
\hline $\mathrm{O}$ & -5.68921900 & -1.39514400 & 0.69837200 \\
\hline $\mathrm{O}$ & -4.43783000 & -2.67719700 & -0.81960000 \\
\hline $\mathrm{O}$ & -4.62589100 & -0.22067400 & -3.00592400 \\
\hline $\mathrm{O}$ & -5.90267200 & 1.00427800 & -1.56018700 \\
\hline
\end{tabular}

\section{INT3A-Rh(II)}

Sum of electronic and zero-point Energies = -2456.306460

Sum of electronic and thermal Free Energies = $-2456.393783$

$\begin{array}{rrrr}\text { Rh } & -0.50951000 & 0.76978500 & 0.65474200 \\ \mathrm{C} & -2.22432100 & -0.57063800 & 2.59767200 \\ \mathrm{C} & -0.10671500 & -1.69624300 & 2.46055500 \\ \mathrm{C} & 1.40185500 & 0.27473600 & -0.41236200 \\ \mathrm{C} & 0.28645900 & -0.31891500 & -1.13023900 \\ \mathrm{C} & -0.48991600 & 0.77539000 & -1.61228000 \\ \mathrm{C} & 0.12805200 & 1.98788600 & -1.24233000 \\ \mathrm{C} & 1.33755800 & 1.71435100 & -0.55882700 \\ \mathrm{C} & 2.53325800 & -0.49833200 & 0.18637600 \\ \mathrm{C} & -2.65905100 & -1.50256700 & 3.54365300 \\ \mathrm{C} & -1.82013300 & -2.53301700 & 3.94596300 \\ \mathrm{C} & -0.54243800 & -2.62958000 & 3.39779600 \\ \mathrm{C} & -1.68722800 & 1.07271900 & -2.44281300 \\ \mathrm{H} & 0.90233200 & -1.79143500 & 2.05680400 \\ \mathrm{H} & -3.66455800 & -1.43276000 & 3.96130100 \\ \mathrm{H} & 0.12003700 & -3.44084800 & 3.70337200 \\ \mathrm{H} & -2.16409900 & -3.26371100 & 4.67734800 \\ \mathrm{H} & 2.17735000 & -1.51897100 & 0.40527900 \\ \mathrm{H} & -1.55190800 & 0.74940800 & -3.49176200 \\ \mathrm{H} & 3.34843900 & -0.62672400 & -0.54310000 \\ \mathrm{H} & -2.62399400 & 0.62496000 & -2.08028500 \\ \mathrm{C} & -0.62296600 & 3.10866100 & -1.87722100 \\ \mathrm{H} & -0.04355400 & 3.57593800 & -2.69735400 \\ \mathrm{H} & -0.91692900 & 3.91786700 & -1.18830700 \\ \mathrm{C} & 3.08988500 & 0.06682000 & 1.47887500 \\ \mathrm{~N} & 4.44804000 & -0.12369400 & 1.71023700 \\ \mathrm{H} & 4.74970100 & 0.40287400 & 2.52388100 \\ \mathrm{C} & 5.45523300 & -0.64405800 & 0.87246000 \\ \mathrm{C} & 6.60171000 & 0.10711500 & 0.62311100 \\ \mathrm{C} & 5.36293100 & -1.92712700 & 0.31759300 \\ \mathrm{C} & 7.63813700 & -0.39260000 & -0.16230600 \\ \mathrm{H} & 6.67724900 & 1.10818300 & 1.04891200\end{array}$

INT3B-Rh(II) 
Sum of electronic and zero-point Energies = $-2456.311608$

Sum of electronic and thermal Free Energies = $-2456.398025$

c

c

c

c

c

c

c $\begin{array}{lll}1.05638700 & -0.08335800 & 0.04490800\end{array}$

$\begin{array}{lll}1.32234600 & 0.08553100 & 2.94094400\end{array}$

$\begin{array}{lll}0.27340400 & 2.07212300 & 2.07579900\end{array}$

$\begin{array}{lll}-0.99382900 & 0.07625300 & -0.78906900\end{array}$

$\begin{array}{lll}-0.14109600 & 1.09073900 & -1.40216000\end{array}$

$\begin{array}{llll}0.76814000 & 0.37420200 & -2.24253200\end{array}$

$\begin{array}{lll}0.51929000 & -0.99495000 & -2.13822400\end{array}$

$-0.61259300 \quad-1.21473400-1.30161200$

$\begin{array}{lll}-2.15338300 & 0.36935400 & 0.11192200\end{array}$

$\begin{array}{lll}1.18088600 & 0.57867100 & 4.23984600\end{array}$

$\begin{array}{llll}0.58541800 & 1.81458200 & 4.46212300\end{array}$

$\begin{array}{lll}0.14101900 & 2.56407400 & 3.37393900\end{array}$

$\begin{array}{lll}1.84168900 & 0.58242400 & -3.25786200\end{array}$

$\begin{array}{lll}-0.06879400 & 2.69135700 & 1.24102300\end{array}$

$\begin{array}{lll}1.54819200 & 0.00165600 & 5.09040500\end{array}$

$\begin{array}{llll}-0.31014000 & 3.54434000 & 3.53712700\end{array}$

$\begin{array}{lll}0.48047700 & 2.19707900 & 5.47714200\end{array}$

$\begin{array}{lll}-1.96535900 & 1.32678900 & 0.62523500\end{array}$

$\begin{array}{llll}1.61388100 & 1.37730800 & -3.98445700\end{array}$

$\begin{array}{llll}-3.07067100 & 0.52053300 & -0.47835200\end{array}$

$\begin{array}{llll}2.81656300 & 0.78762500 & -2.78188500\end{array}$

$\begin{array}{lll}1.38253100 & -1.71012200 & -3.12613800\end{array}$

$\begin{array}{lll}0.82280000 & -2.41672600 & -3.75930900\end{array}$

$2.22322800 \quad-2.23793900 \quad-2.64466400$

$\begin{array}{lll}-2.42395000 & -0.64521400 & 1.20791400\end{array}$

$\begin{array}{lll}-3.75781600 & -0.80527600 & 1.57823300\end{array}$

$\begin{array}{lll}-3.86074000 & -1.59348400 & 2.20932500\end{array}$

$\begin{array}{lll}-4.93264000 & -0.28690100 & 0.99692300\end{array}$

$\begin{array}{lll}-5.93226300 & -1.15400800 & 0.56138200\end{array}$

$\begin{array}{lll}-5.14854600 & 1.09281400 & 0.88293000\end{array}$

$\begin{array}{lll}-7.12274500 & -0.67318600 & 0.02015800\end{array}$

$\begin{array}{lll}-5.76658800 & -2.22890800 & 0.64103500\end{array}$

$\begin{array}{lll}-6.31439500 & 1.57924200 & 0.31903800\end{array}$

$\begin{array}{lll}-4.38953900 & 1.78272200 & 1.24942400\end{array}$

$\begin{array}{lll}-7.31230700 & 0.70105900 & -0.11298100\end{array}$

$\begin{array}{lll}-7.88122700 & -1.38039800 & -0.30695200\end{array}$

$\begin{array}{lll}-6.48819000 & 2.64865500 & 0.21689000\end{array}$

$\begin{array}{lll}1.88040100 & -0.66291300 & -3.95797700\end{array}$

$\begin{array}{lll}-1.24924900 & -2.50684300 & -1.06062900\end{array}$

$-2.64464600 \quad-2.64368600 \quad-1.07137300$

$-0.47471700 \quad-3.65970800 \quad-0.87128400$

$\begin{array}{lll}-3.24372300 & -3.88179000 & -0.87341300\end{array}$

$-3.26701100-1.76829200 \quad-1.26402300$

$\begin{array}{lll}-1.07381900 & -4.89743500 & -0.68000100\end{array}$

$\begin{array}{lll}0.61133900 & -3.56383300 & -0.84645900\end{array}$

$\begin{array}{llll}-2.46207100 & -5.01645700 & -0.67492200\end{array}$

$\begin{array}{lll}-4.33142800 & -3.96294700 & -0.89528000\end{array}$

$-0.45050400 \quad-5.77737200 \quad-0.52375800$

$\begin{array}{lll}-2.93020900 & -5.98826700 & -0.52198600\end{array}$

$\begin{array}{lll}-0.27375800 & 2.55191800 & -1.37251500\end{array}$

$\begin{array}{llll}0.88002400 & 3.34581800 & -1.46650400\end{array}$

$\begin{array}{llll}-1.51451700 & 3.19744900 & -1.28640700\end{array}$

$\begin{array}{llll}0.79291100 & 4.73076000 & -1.44711800\end{array}$

$\begin{array}{lll}1.85548000 & 2.85517000 & -1.49908200\end{array}$

$\begin{array}{lll}-1.59769300 & 4.58692000 & -1.26073300\end{array}$

$\begin{array}{lll}-2.43042100 & 2.60720400 & -1.25706600\end{array}$

$\begin{array}{lll}-0.44553900 & 5.36146200 & -1.33733400\end{array}$

$\begin{array}{llll}1.70721100 & 5.32213200 & -1.50088000\end{array}$

$\begin{array}{llll}-2.57466700 & 5.06518500 & -1.18892300\end{array}$

$\begin{array}{lll}-0.51042100 & 6.44877600 & -1.31211600\end{array}$

$\begin{array}{lll}-8.42511800 & 1.28034300 & -0.63938500\end{array}$

$\begin{array}{llll}-9.43674800 & 0.43131000 & -1.10899900\end{array}$

$\begin{array}{lrrr}\mathrm{H} & -10.22800300 & 1.07682500 & -1.50064500 \\ \mathrm{H} & -9.07603500 & -0.22486700 & -1.91669700 \\ \mathrm{H} & -9.85124400 & -0.19533900 & -0.30322600 \\ \mathrm{C} & 1.99845600 & -1.16929200 & 2.62540900 \\ \mathrm{C} & 2.53339400 & -2.06471000 & 3.54798600 \\ \mathrm{C} & 3.19687500 & -3.19865400 & 3.09859700 \\ \mathrm{H} & 2.42972800 & -1.87046100 & 4.61357900 \\ \mathrm{C} & 2.79863400 & -2.47333800 & 0.86081700 \\ \mathrm{C} & 3.34615300 & -3.40477900 & 1.73414400 \\ \mathrm{H} & 3.61568100 & -3.90498300 & 3.81468100 \\ \mathrm{H} & 2.93875300 & -2.53677400 & -0.22000700 \\ \mathrm{H} & 3.90552100 & -4.24888100 & 1.33912200 \\ \mathrm{~N} & 2.10936400 & -1.41428100 & 1.29454500 \\ \mathrm{O} & -1.57634000 & -1.29021600 & 1.78640700 \\ \mathrm{C} & 0.85282500 & 0.82195500 & 1.82600800 \\ \mathrm{C} & 3.96453400 & 2.34866800 & 1.98426200 \\ \mathrm{C} & 3.52183500 & 3.01909000 & 0.67594100 \\ \mathrm{H} & 3.15110300 & 2.31970200 & 2.72627000 \\ \mathrm{H} & 4.82710300 & 2.85613900 & 2.44568400 \\ \mathrm{H} & 2.46760000 & 3.33630900 & 0.71963200 \\ \mathrm{H} & 4.13068100 & 3.90152200 & 0.42013900 \\ \mathrm{C} & 5.85217200 & -0.06585600 & -2.58496200 \\ \mathrm{C} & 4.84650100 & -1.18531500 & -2.80402600 \\ \mathrm{H} & 5.39760300 & 0.90132900 & -2.90361600 \\ \mathrm{H} & 6.78743200 & -0.19789100 & -3.15989700 \\ \mathrm{H} & 4.27297200 & -1.10238800 & -3.74208300 \\ \mathrm{H} & 5.38167400 & -2.16079300 & -2.80533700 \\ \mathrm{~B} & 4.16555800 & 0.84851900 & 0.25509400 \\ \mathrm{~B} & 4.83315000 & -0.54273900 & -0.57238600 \\ \mathrm{O} & 4.32681500 & 1.03494000 & 1.62278000 \\ \mathrm{O} & 3.68091100 & 2.02974700 & -0.32548400 \\ \mathrm{O} & 6.09949100 & -0.10013000 & -1.22162400 \\ \mathrm{O} & 3.99133700 & -1.06646100 & -1.70369300 \\ \mathrm{H} & 5.08556000 & -1.42782900 & 0.26624600\end{array}$

\section{TS2B-Rh(II)}

Sum of electronic and zero-point Energies = $-2456.311479$

Sum of electronic and thermal Free Energies = $-2456.396988$

\begin{tabular}{crrr}
$\mathrm{Rh}$ & 1.09553700 & -0.09510500 & 0.03551200 \\
$\mathrm{C}$ & 1.24193000 & 0.12512400 & 2.93567300 \\
$\mathrm{C}$ & 0.31489200 & 2.13286000 & 1.98540000 \\
$\mathrm{C}$ & -0.94754900 & 0.06615300 & -0.85275800 \\
$\mathrm{C}$ & -0.07216100 & 1.07529100 & -1.44437600 \\
$\mathrm{C}$ & 0.84964700 & 0.35144600 & -2.26395200 \\
$\mathrm{C}$ & 0.57912300 & -1.01405700 & -2.17585000 \\
$\mathrm{C}$ & -0.57475600 & -1.22485700 & -1.36754200 \\
$\mathrm{C}$ & -2.11224000 & 0.36875800 & 0.03864400 \\
$\mathrm{C}$ & 1.06683500 & 0.65207800 & 4.21643800 \\
$\mathrm{C}$ & 0.51130800 & 1.91438600 & 4.38880000 \\
$\mathrm{C}$ & 0.14311500 & 2.65561800 & 3.26727200 \\
$\mathrm{C}$ & 1.95280100 & 0.55331400 & -3.24726900 \\
$\mathrm{H}$ & 0.03547900 & 2.74841900 & 1.12570500 \\
$\mathrm{H}$ & 1.37337400 & 0.07857800 & 5.09312700 \\
$\mathrm{H}$ & -0.27742300 & 3.65523900 & 3.39024300 \\
$\mathrm{H}$ & 0.37763100 & 2.32219600 & 5.39051500 \\
$\mathrm{H}$ & -1.92659400 & 1.33318200 & 0.53996400 \\
$\mathrm{H}$ & 1.74954500 & 1.34851700 & -3.98146600 \\
$\mathrm{H}$ & -3.03022500 & 0.50999600 & -0.55363200 \\
$\mathrm{H}$ & 2.91292600 & 0.75878300 & -2.74311800 \\
$\mathrm{C}$ & 1.44214700 & -1.73571200 & -3.15756900 \\
$\mathrm{H}$ & 0.86816000 & -2.39859200 & -3.82588600 \\
$\mathrm{H}$ & 2.24123100 & -2.32030000 & -2.67384100 \\
$\mathrm{C}$ & -2.38359500 & -0.63021600 & 1.14847100 \\
$\mathrm{~N}$ & -3.71355200 & -0.76372200 & 1.54479500 \\
& & & \\
\hline & & & \\
$\mathrm{N}$ & & &
\end{tabular}




\begin{tabular}{|c|c|c|c|}
\hline $\mathrm{H}$ & -3.81280200 & -1.54088000 & 2.19009600 \\
\hline $\mathrm{C}$ & -4.89687500 & -0.24710000 & 0.98226300 \\
\hline $\mathrm{C}$ & -5.93423900 & -1.11451000 & 0.64573900 \\
\hline $\mathrm{C}$ & -5.09075000 & 1.12822300 & 0.79584100 \\
\hline $\mathrm{C}$ & -7.13900000 & -0.64004200 & 0.13193200 \\
\hline $\mathrm{H}$ & -5.78911300 & -2.18624600 & 0.78455700 \\
\hline $\mathrm{C}$ & -6.27259200 & 1.60603900 & 0.25782800 \\
\hline $\mathrm{H}$ & -4.30487000 & 1.82351700 & 1.08743600 \\
\hline $\mathrm{C}$ & -7.30751900 & 0.72769000 & -0.07454900 \\
\hline $\mathrm{H}$ & -7.92592400 & -1.34854500 & -0.11551600 \\
\hline $\mathrm{H}$ & -6.42960800 & 2.67147800 & 0.10137900 \\
\hline $\mathrm{O}$ & 2.01468400 & -0.69265000 & -3.94374800 \\
\hline $\mathrm{C}$ & -1.21984600 & -2.51438900 & -1.14388700 \\
\hline $\mathrm{C}$ & -2.61635100 & -2.64369700 & -1.11169400 \\
\hline $\mathrm{C}$ & -0.44938400 & -3.67952300 & -1.01846600 \\
\hline $\mathrm{C}$ & -3.21683100 & -3.88401100 & -0.93545900 \\
\hline $\mathrm{H}$ & -3.24050000 & -1.76027100 & -1.25444800 \\
\hline $\mathrm{C}$ & -1.05002900 & -4.91920300 & -0.84719100 \\
\hline $\mathrm{H}$ & 0.63738100 & -3.59245000 & -1.03171400 \\
\hline $\mathrm{C}$ & -2.43800000 & -5.03013500 & -0.79998100 \\
\hline $\mathrm{H}$ & -4.30493600 & -3.95773300 & -0.92424800 \\
\hline $\mathrm{H}$ & -0.42764100 & -5.80736300 & -0.74149300 \\
\hline $\mathrm{H}$ & -2.90822600 & -6.00340600 & -0.66372500 \\
\hline $\mathrm{C}$ & -0.21466900 & 2.53563600 & -1.44050600 \\
\hline $\mathrm{C}$ & 0.93388600 & 3.33845100 & -1.52146000 \\
\hline $\mathrm{C}$ & -1.46263700 & 3.17201200 & -1.39789100 \\
\hline $\mathrm{C}$ & 0.83356600 & 4.72276900 & -1.53504400 \\
\hline $\mathrm{H}$ & 1.91338900 & 2.85500000 & -1.51874400 \\
\hline $\mathrm{C}$ & -1.55891900 & 4.56078700 & -1.40220100 \\
\hline $\mathrm{H}$ & -2.37381200 & 2.57401500 & -1.38043600 \\
\hline $\mathrm{C}$ & -0.41246100 & 5.34457800 & -1.46810300 \\
\hline $\mathrm{H}$ & 1.74324300 & 5.32191000 & -1.58207700 \\
\hline $\mathrm{H}$ & -2.54172200 & 5.03101100 & -1.36376500 \\
\hline $\mathrm{H}$ & -0.48752000 & 6.43155200 & -1.46823700 \\
\hline $\mathrm{O}$ & -8.43315900 & 1.30015300 & -0.58190500 \\
\hline $\mathrm{C}$ & -9.48455200 & 0.44778200 & -0.94549300 \\
\hline $\mathrm{H}$ & -10.28268500 & 1.08613000 & -1.33500700 \\
\hline $\mathrm{H}$ & -9.18024700 & -0.26451100 & -1.72860800 \\
\hline $\mathrm{H}$ & -9.86817300 & -0.11942900 & -0.08235400 \\
\hline $\mathrm{C}$ & 1.86884200 & -1.16609600 & 2.67558600 \\
\hline $\mathrm{C}$ & 2.33316300 & -2.06135000 & 3.63664600 \\
\hline $\mathrm{C}$ & 2.94648700 & -3.24061600 & 3.23649800 \\
\hline $\mathrm{H}$ & 2.21227600 & -1.83187400 & 4.69341100 \\
\hline $\mathrm{C}$ & 2.64761400 & -2.55777900 & 0.97106700 \\
\hline $\mathrm{C}$ & 3.11774400 & -3.49468900 & 1.88208100 \\
\hline $\mathrm{H}$ & 3.30781900 & -3.94772900 & 3.98252700 \\
\hline $\mathrm{H}$ & 2.80320400 & -2.65879700 & -0.10365700 \\
\hline $\mathrm{H}$ & 3.63421700 & -4.38251700 & 1.52624800 \\
\hline $\mathrm{N}$ & 2.00707100 & -1.45133600 & 1.35657700 \\
\hline $\mathrm{O}$ & -1.53985100 & -1.28658300 & 1.71966200 \\
\hline $\mathrm{C}$ & 0.85364900 & 0.85538600 & 1.78528500 \\
\hline $\mathrm{C}$ & 3.89483000 & 2.44853300 & 1.97764500 \\
\hline $\mathrm{C}$ & 3.63912500 & 3.11244800 & 0.61709600 \\
\hline $\mathrm{H}$ & 3.00914100 & 2.49916800 & 2.63042200 \\
\hline $\mathrm{H}$ & 4.74180600 & 2.90345700 & 2.51478700 \\
\hline $\mathrm{H}$ & 2.65371800 & 3.60469700 & 0.57894400 \\
\hline $\mathrm{H}$ & 4.40315400 & 3.86512900 & 0.36247300 \\
\hline $\mathrm{C}$ & 5.90432500 & -0.15050700 & -2.33700200 \\
\hline $\mathrm{C}$ & 4.91682500 & -1.27273000 & -2.61976900 \\
\hline $\mathrm{H}$ & 5.48800700 & 0.81241300 & -2.71222500 \\
\hline $\mathrm{H}$ & 6.88590700 & -0.29825000 & -2.82304800 \\
\hline $\mathrm{H}$ & 4.41353500 & -1.19444000 & -3.59741200 \\
\hline $\mathrm{H}$ & 5.44897100 & -2.24856800 & -2.57741800 \\
\hline $\mathrm{B}$ & 4.03770700 & 0.86660500 & 0.32061500 \\
\hline B & 4.71506500 & -0.58780600 & -0.41208700 \\
\hline $\mathrm{O}$ & 4.18846600 & 1.10204400 & 1.68106100 \\
\hline
\end{tabular}

$\begin{array}{lrrr}\mathrm{O} & 3.68432200 & 2.05895900 & -0.32795100 \\ \mathrm{O} & 6.03166100 & -0.15774800 & -0.95504500 \\ \mathrm{O} & 3.98257500 & -1.14946800 & -1.58705900 \\ \mathrm{H} & 4.88927400 & -1.42388200 & 0.49070600\end{array}$

\section{INT4-Rh(II)}

Sum of electronic and zero-point Energies = $-2456.358443$

Sum of electronic and thermal Free Energies = $-2456.447588$

\begin{tabular}{|c|c|c|c|}
\hline $\mathrm{Rh}$ & 1.29218000 & 0.04459500 & -0.01328400 \\
\hline $\mathrm{C}$ & 1.91388200 & -0.07586800 & 2.89913000 \\
\hline $\mathrm{C}$ & 1.40927200 & 2.17968200 & 2.23732400 \\
\hline $\mathrm{C}$ & -1.39553500 & 0.60283700 & -1.05929700 \\
\hline $\mathrm{C}$ & -0.40333700 & 1.58387800 & -1.35534800 \\
\hline $\mathrm{C}$ & 0.69299600 & 0.89268800 & -1.97001800 \\
\hline $\mathrm{C}$ & 0.34786800 & -0.48222600 & -2.07863400 \\
\hline $\mathrm{C}$ & -0.98541600 & -0.65699500 & -1.56795800 \\
\hline $\mathrm{C}$ & -2.50505900 & 0.82077700 & -0.07232000 \\
\hline $\mathrm{C}$ & 1.84290300 & 0.30948400 & 4.24187300 \\
\hline $\mathrm{C}$ & 1.55893500 & 1.62416000 & 4.58489700 \\
\hline $\mathrm{C}$ & 1.34249800 & 2.56074800 & 3.57354100 \\
\hline $\mathrm{C}$ & 1.70051700 & 1.19937400 & -3.03265800 \\
\hline $\mathrm{H}$ & 1.24876600 & 2.92943400 & 1.45994900 \\
\hline $\mathrm{H}$ & 1.97706600 & -0.43273300 & 5.03162100 \\
\hline $\mathrm{H}$ & 1.12103900 & 3.59788200 & 3.83125300 \\
\hline $\mathrm{H}$ & 1.49744500 & 1.91624300 & 5.63342400 \\
\hline $\mathrm{H}$ & -2.36834200 & 1.80987000 & 0.39541000 \\
\hline $\mathrm{H}$ & 1.23831200 & 1.76527900 & -3.86755600 \\
\hline $\mathrm{H}$ & -3.50368100 & 0.84630800 & -0.53417300 \\
\hline $\mathrm{H}$ & 2.58812200 & 1.75440900 & -2.69690400 \\
\hline $\mathrm{C}$ & 1.12416400 & -1.04987000 & -3.22530400 \\
\hline $\mathrm{H}$ & 0.47758500 & -1.18829200 & -4.11750400 \\
\hline $\mathrm{H}$ & 1.63340200 & -2.00964400 & -3.04326600 \\
\hline $\mathrm{C}$ & -2.48355700 & -0.17912600 & 1.07090000 \\
\hline $\mathrm{N}$ & -3.70700200 & -0.54937700 & 1.62598700 \\
\hline $\mathrm{H}$ & -3.58358400 & -1.32930900 & 2.26380800 \\
\hline $\mathrm{C}$ & -5.02033300 & -0.28384800 & 1.19531300 \\
\hline $\mathrm{C}$ & -5.90008900 & -1.33984300 & 0.96531200 \\
\hline $\mathrm{C}$ & -5.49497100 & 1.02496100 & 1.04141300 \\
\hline $\mathrm{C}$ & -7.22039900 & -1.11274200 & 0.58321600 \\
\hline $\mathrm{H}$ & -5.53357500 & -2.36105000 & 1.07154400 \\
\hline $\mathrm{C}$ & -6.79686900 & 1.25972700 & 0.63446200 \\
\hline $\mathrm{H}$ & -4.82977000 & 1.86035200 & 1.25395300 \\
\hline $\mathrm{C}$ & -7.67060900 & 0.19408600 & 0.40539500 \\
\hline $\mathrm{H}$ & -7.87649400 & -1.96337800 & 0.41410000 \\
\hline $\mathrm{H}$ & -7.17221000 & 2.27284200 & 0.50309500 \\
\hline $\mathrm{O}$ & 2.13847800 & -0.08269700 & -3.49343000 \\
\hline $\mathrm{C}$ & -1.68893700 & -1.92226200 & -1.50774000 \\
\hline $\mathrm{C}$ & -3.09308000 & -2.01269200 & -1.43637400 \\
\hline $\mathrm{C}$ & -0.97761700 & -3.13711800 & -1.56725000 \\
\hline $\mathrm{C}$ & -3.74040100 & -3.24080900 & -1.40508800 \\
\hline $\mathrm{H}$ & -3.68965200 & -1.10083800 & -1.44701500 \\
\hline $\mathrm{C}$ & -1.62510000 & -4.36264400 & -1.54115800 \\
\hline $\mathrm{H}$ & 0.11016400 & -3.09556400 & -1.61808300 \\
\hline $\mathrm{C}$ & -3.01544300 & -4.43002000 & -1.45417300 \\
\hline $\mathrm{H}$ & -4.83097100 & -3.26458900 & -1.36181200 \\
\hline $\mathrm{H}$ & -1.03658700 & -5.27984200 & -1.58135700 \\
\hline $\mathrm{H}$ & -3.52456500 & -5.39308200 & -1.43240800 \\
\hline $\mathrm{C}$ & -0.48949500 & 3.02588500 & -1.17121800 \\
\hline $\mathrm{C}$ & 0.67185900 & 3.80485500 & -1.00271800 \\
\hline $\mathrm{C}$ & -1.72011900 & 3.70547900 & -1.22962700 \\
\hline $\mathrm{C}$ & 0.59536200 & 5.18528300 & -0.87974900 \\
\hline $\mathrm{H}$ & 1.63811000 & 3.30308300 & -0.93003000 \\
\hline $\mathrm{C}$ & -1.79196900 & 5.08708300 & -1.09533700 \\
\hline $\mathrm{H}$ & -2.63016000 & 3.13895900 & -1.42763400 \\
\hline
\end{tabular}


$\begin{array}{lll}-0.63518000 & 5.84037800 & -0.91772400\end{array}$

$\begin{array}{lll}1.51281100 & 5.75794700 & -0.74044400\end{array}$

$\begin{array}{llll}-2.76305400 & 5.58101100 & -1.14866600\end{array}$

$\begin{array}{llll}-0.68997300 & 6.92397200 & -0.81542700\end{array}$

$\begin{array}{lll}-8.93365000 & 0.53243100 & 0.02195100\end{array}$

$\begin{array}{lll}-9.82908600 & -0.51158500 & -0.24295500\end{array}$

$\begin{array}{lll}-10.77109000 & -0.04534100 & -0.54621100\end{array}$

$-9.47103100-1.16022900-1.05818100$

$\begin{array}{lll}-10.00840800 & -1.13382300 & 0.64866600\end{array}$

$\begin{array}{lll}2.20894200 & -1.45173700 & 2.50163400\end{array}$

$\begin{array}{lll}2.84010600 & -2.38707700 & 3.32954000\end{array}$

$\begin{array}{lll}3.12224700 & -3.65411400 & 2.84771700\end{array}$

$\begin{array}{lll}3.13454700 & -2.10146200 & 4.33788200\end{array}$

$\begin{array}{lll}2.16936100 & -2.98823800 & 0.76992700\end{array}$

$\begin{array}{llll}2.78413800 & -3.96839700 & 1.53377300\end{array}$

$3.62041500-4.38570700 \quad 3.48340100$

$\begin{array}{lll}1.89614400 & -3.17901800 & -0.26938300\end{array}$

$\begin{array}{llll}2.99872700 & -4.94402800 & 1.10368400\end{array}$

$\begin{array}{lll}1.87011400 & -1.77294500 & 1.23129100\end{array}$

$\begin{array}{lll}-1.46764600 & -0.64566000 & 1.54363400\end{array}$

$\begin{array}{lll}1.68596900 & 0.85713300 & 1.85478800\end{array}$

$\begin{array}{lll}5.20773000 & 1.16607200 & 1.01470400\end{array}$

$\begin{array}{lll}4.84986400 & 2.30121900 & 0.06233500\end{array}$

$\begin{array}{llll}5.12727900 & 1.48916400 & 2.06880500\end{array}$

$\begin{array}{llll}6.21303800 & 0.75954600 & 0.84224700\end{array}$

$\begin{array}{llll}5.05502200 & 3.29752400 & 0.47877100\end{array}$

$\begin{array}{lll}5.39300900 & 2.20509300 & -0.89345100\end{array}$

$\begin{array}{lll}4.94284400 & -0.43580400 & -2.33988400\end{array}$

$\begin{array}{lll}4.15714300 & -1.69266300 & -1.94323900\end{array}$

$\begin{array}{llll}4.38563900 & 0.47999300 & -2.10967100\end{array}$

$\begin{array}{llll}5.21776900 & -0.41585400 & -3.40316200\end{array}$

$\begin{array}{lll}3.24398700 & -1.42734700 & -1.38624800\end{array}$

$\begin{array}{lll}3.89336700 & -2.32052900 & -2.80450300\end{array}$

$\begin{array}{lll}3.08665400 & 0.85147900 & 0.27396300\end{array}$

$\begin{array}{lll}6.09682400 & -1.62671700 & -0.82504300\end{array}$

$\begin{array}{lll}4.22946400 & 0.18126800 & 0.75543300\end{array}$

$\begin{array}{lll}3.46086000 & 2.14071900 & -0.15390100\end{array}$

$6.13623600-0.47321000-1.55095400$

$\begin{array}{lll}5.03257400 & -2.42829600 & -1.08190700\end{array}$

$6.96759300-1.92869700-0.07148000$

\section{INT5}

Sum of electronic and zero-point Energies = $-2201.947439$

Sum of electronic and thermal Free Energies = $-2202.024934$

$\begin{array}{crrr}\mathrm{Rh} & 1.62040900 & 0.08494100 & -0.16123000 \\ \mathrm{C} & 3.35391100 & -1.32255200 & 0.24766900 \\ \mathrm{C} & 3.79026900 & 0.02680300 & 0.51514500 \\ \mathrm{C} & 3.90244100 & -1.93777700 & -0.92070200 \\ \mathrm{C} & -0.48933500 & 0.52107100 & -0.71831400 \\ \mathrm{C} & 0.31446100 & 1.62614600 & -1.29415400 \\ \mathrm{C} & 1.15910600 & 1.02328800 & -2.23003200 \\ \mathrm{C} & 0.92757800 & -0.38648400 & -2.22710200 \\ \mathrm{C} & -0.22709500 & -0.68863600 & -1.44234300 \\ \mathrm{C} & -1.62637700 & 0.76474300 & 0.22882600 \\ \mathrm{C} & 4.76907500 & 0.64631800 & -0.30832600 \\ \mathrm{C} & 5.25544900 & 0.00373500 & -1.41744800 \\ \mathrm{C} & 4.81389800 & -1.30521900 & -1.72584800 \\ \mathrm{C} & 2.22804800 & 1.33049800 & -3.21988500 \\ \mathrm{H} & 3.59248700 & -2.95950500 & -1.14717400 \\ \mathrm{H} & 5.10304100 & 1.65299600 & -0.05247800 \\ \mathrm{H} & 5.20823000 & -1.81071500 & -2.60622500 \\ \mathrm{H} & 5.98276400 & 0.49585900 & -2.06108100 \\ \mathrm{H} & -1.48104000 & 1.74211200 & 0.71681300 \\ \mathrm{H} & 1.86060000 & 1.96923200 & -4.04354800\end{array}$

$\begin{array}{llll}-2.56398300 & 0.87232500 & -0.33646900\end{array}$

$\begin{array}{llll}3.11132700 & 1.82319500 & -2.78148800\end{array}$

$\begin{array}{lll}1.73931900 & -0.97748200 & -3.33028200\end{array}$

$\begin{array}{lll}1.09309200 & -1.28701500 & -4.17334900\end{array}$

$\begin{array}{lll}2.35050100 & -1.84281400 & -3.03810300\end{array}$

$\begin{array}{lll}-1.79413100 & -0.25319700 & 1.34119700\end{array}$

$\begin{array}{lll}-3.07689600 & -0.51499200 & 1.76650800\end{array}$

$\begin{array}{lll}-3.09316400 & -1.26127700 & 2.45486500\end{array}$

$\begin{array}{lll}-4.32135800 & -0.09910700 & 1.23149300\end{array}$

$\begin{array}{lll}-5.25168800 & -1.05565600 & 0.83467400\end{array}$

$\begin{array}{lll}-4.66433300 & 1.25419200 & 1.14299000\end{array}$

$\begin{array}{lll}-6.50219100 & -0.68470700 & 0.34815300\end{array}$

$\begin{array}{lll}-4.98662000 & -2.11088400 & 0.90598800\end{array}$

$\begin{array}{llll}-5.89346400 & 1.63396700 & 0.63465200\end{array}$

$\begin{array}{lll}-3.95853300 & 2.00968500 & 1.48528100\end{array}$

$\begin{array}{lll}-6.82385600 & 0.66839300 & 0.23639000\end{array}$

$\begin{array}{lll}-7.20704300 & -1.45694400 & 0.05095500\end{array}$

$\begin{array}{lll}-6.17124800 & 2.68251400 & 0.55143800\end{array}$

$\begin{array}{lll}2.62603900 & 0.06185400 & -3.72850500\end{array}$

$\begin{array}{lll}-0.90397000 & -1.98099900 & -1.37757000\end{array}$

$\begin{array}{lll}-2.28251100 & -2.08932800 & -1.13961000\end{array}$

$-0.18774600 \quad-3.16391900-1.61893700$

$\begin{array}{lll}-2.90843600 & -3.33012300 & -1.10672800\end{array}$

$-2.88660000-1.19086500-1.01536100$

$-0.81468900-4.40097700-1.59036600$

$\begin{array}{lll}0.88778500 & -3.10660000 & -1.77428500\end{array}$

$-2.17947300 \quad-4.49491700 \quad-1.32553600$

$\begin{array}{lll}-3.98404000 & -3.38168400 & -0.93470600\end{array}$

$\begin{array}{lll}-0.22794400 & -5.30220400 & -1.76429200\end{array}$

$\begin{array}{lll}-2.67107900 & -5.46620400 & -1.30125800\end{array}$

$\begin{array}{llll}0.23437400 & 3.06065100 & -0.99259300\end{array}$

$\begin{array}{lll}0.15021600 & 3.97740800 & -2.04945500\end{array}$

$\begin{array}{lll}0.24650400 & 3.56318800 & 0.31616300\end{array}$

$\begin{array}{llll}0.07612000 & 5.34404800 & -1.80864700\end{array}$

$\begin{array}{llll}0.12593900 & 3.60093500 & -3.07256300\end{array}$

$\begin{array}{llll}0.15926300 & 4.92916700 & 0.55680200\end{array}$

$\begin{array}{lll}0.37517700 & 2.87312200 & 1.15037600\end{array}$

$\begin{array}{llll}0.07226900 & 5.82699400 & -0.50334500\end{array}$

$\begin{array}{llll}0.01194900 & 6.03593300 & -2.64740800\end{array}$

$\begin{array}{lll}0.17814600 & 5.29612100 & 1.58232200\end{array}$

$\begin{array}{llll}0.00924200 & 6.89718300 & -0.31347900\end{array}$

$\begin{array}{llll}-8.00127700 & 1.14337000 & -0.23350800\end{array}$

$\begin{array}{lll}-8.96672200 & 0.21066900 & -0.65077800\end{array}$

$\begin{array}{llll}-9.82714800 & 0.79018100 & -0.99460800\end{array}$

$\begin{array}{lll}-8.59699000 & -0.41134100 & -1.48005900\end{array}$

$\begin{array}{lll}-9.28132100 & -0.44433900 & 0.17601000\end{array}$

$\begin{array}{lll}3.35806300 & 0.64557600 & 1.78999700\end{array}$

$\begin{array}{lll}4.13725000 & 1.11462500 & 2.83464800\end{array}$

$\begin{array}{lll}3.48787500 & 1.60518900 & 3.96646000\end{array}$

$\begin{array}{llll}5.22249300 & 1.07575800 & 2.76338200\end{array}$

$\begin{array}{lll}1.38774900 & 1.11591600 & 2.91949500\end{array}$

$\begin{array}{llll}2.09950400 & 1.58991900 & 4.01806200\end{array}$

$\begin{array}{llll}4.06470500 & 1.98180900 & 4.80937700\end{array}$

$\begin{array}{lll}0.30031300 & 1.06188500 & 2.90025300\end{array}$

$\begin{array}{lll}1.56552400 & 1.94133500 & 4.89775800\end{array}$

$\begin{array}{llll}2.01491900 & 0.68331400 & 1.82871200\end{array}$

$\begin{array}{llll}0.80460900 & -3.64939100 & 1.63199600\end{array}$

$\begin{array}{lll}1.27354500 & -2.97335200 & 2.92858300\end{array}$

$-0.18331100 \quad-3.27641200 \quad 1.31897300$

$\begin{array}{lll}0.76301300 & -4.74436100 & 1.70096900\end{array}$

$\begin{array}{lll}0.44822100 & -2.47237400 & 3.44972200\end{array}$

$\begin{array}{lll}1.75969000 & -3.67667400 & 3.62152300\end{array}$

$\begin{array}{lll}2.42499700 & -2.16761000 & 1.16421000\end{array}$

$\begin{array}{lll}1.76570200 & -3.26999500 & 0.65845100\end{array}$

$\begin{array}{lll}2.22282200 & -2.00366900 & 2.51504700\end{array}$

$\begin{array}{lll}-0.85389000 & -0.79338900 & 1.90318300\end{array}$ 


\section{TS3}

Sum of electronic and zero-point Energies = $-2201.936448$

Sum of electronic and thermal Free Energies = $-2202.014659$

$\begin{array}{llll}\text { C } & 3.72135300 & -0.41802300 & -0.12355200\end{array}$

$\begin{array}{llll}\mathrm{C} & 4.04971500 & 0.76611500 & 0.58855100\end{array}$

C $\quad 4.37639300 \quad-0.64653600 \quad-1.34591900$

$\begin{array}{llll}\text { C } & -0.66591800 & 0.27218100 & -0.52069600\end{array}$

$\begin{array}{lllll}\text { C } & 0.02470100 & 1.32943900 & -1.30417200\end{array}$

$\begin{array}{lllll}\text { C } & 0.83330900 & 0.64728700 & -2.20295600\end{array}$

$\begin{array}{llll}\text { C } & 0.66837500 & -0.76663600 & -2.01934500\end{array}$

$\begin{array}{lllll}\text { C } & & -0.40801200 & -1.00839100 & -1.12300500\end{array}$

$\begin{array}{llll}\text { C } & -1.79846300 & 0.61056900 & 0.40585900\end{array}$

$\begin{array}{llll}\mathrm{C} & 5.00540800 & 1.65278600 & 0.09221000\end{array}$

$\begin{array}{lllll}\text { C } & 5.64590000 & 1.38307400 & -1.11151600\end{array}$

C $\quad \begin{array}{llll}\text { C } & 5.33417100 & 0.23199900 & -1.83320200\end{array}$

$\begin{array}{lllll}\mathrm{C} & 1.84653400 & 0.86408900 & -3.27374100\end{array}$

$\mathrm{H} \quad 4.13352800 \quad-1.54676700 \quad-1.90673400$

$\begin{array}{llll}\mathrm{H} & 5.21322300 & 2.58026500 & 0.62575500\end{array}$

$\mathrm{H} \quad \begin{array}{llll}\mathrm{H} & 5.82675400 & 0.02952700 & -2.78258700\end{array}$

$\mathrm{H} \quad \begin{array}{lllll}6.38334000 & 2.08519300 & -1.49829500\end{array}$

$\begin{array}{llll}\mathrm{H} & -1.59953100 & 1.58656200 & 0.87709900\end{array}$

$\mathrm{H} \quad \begin{array}{llll}\mathrm{H} & 1.40150100 & 1.33561300 & -4.16908500\end{array}$

$\begin{array}{lllll}\mathrm{H} & -2.71038600 & 0.77068500 & -0.19005100\end{array}$

$\begin{array}{llll}\mathrm{H} & 2.70466900 & 1.47952300 & -2.96028900\end{array}$

C $\quad 1.45279300 \quad-1.45248700 \quad-3.08713200$

$\mathrm{H} \quad 0.79178200 \quad-1.83565000 \quad-3.88752200$

$\mathrm{H} \quad 2.07317100 \quad-2.28638200 \quad-2.73076100$

$\begin{array}{llll}\mathrm{C} & -2.06772500 & -0.36630100 & 1.53101000\end{array}$

N $\quad-3.38088800 \quad-0.57019900 \quad 1.89317300$

$\mathrm{H} \quad-3.45958700 \quad-1.30440300 \quad 2.59038000$

$\begin{array}{llll}\text { C } & -4.57971600 & -0.10086500 & 1.30408200\end{array}$

$\begin{array}{llll}\text { C } & -5.54674600 & -1.01388000 & 0.89262000\end{array}$

$\begin{array}{llll}\text { C } & -4.84557800 & 1.26694100 & 1.17616600\end{array}$

$\begin{array}{llll}\text { C } & -6.75828000 & -0.58649000 & 0.35567800\end{array}$

$\begin{array}{llll}\mathrm{H} & -5.34367200 & -2.08022200 & 0.99566800\end{array}$

$\begin{array}{llll}\text { C } & -6.03390100 & 1.70125500 & 0.61738600\end{array}$

$\begin{array}{llll}\mathrm{H} & -4.11229600 & 1.99067600 & 1.52909800\end{array}$

$\mathrm{C}$

$\mathrm{H}$

$\mathrm{H}$

$\mathrm{O}$

$\mathrm{C}$

C

$\mathrm{C}$

C

$\mathrm{H}$

$\mathrm{C}$

$\mathrm{H}$

C

$\mathrm{H}$

$\mathrm{H}$

$\mathrm{H}$

C

C

C

$\mathrm{C}$

$\mathrm{H}$

C

$\mathrm{H}$

C

$\mathrm{H}$

$\mathrm{H}$

$\begin{array}{lll}-4.11229600 & 1.99067600 & 1.52909800 \\ -7.00184200 & 0.77901400 & 0.20635700\end{array}$

$\begin{array}{lll}-7.49370200 & -1.32593800 & 0.04919800\end{array}$ $\begin{array}{lll}-6.25090900 & 2.76130200 & 0.50426800\end{array}$

$\begin{array}{lll}2.32148400 & -0.44063900 & -3.59495000\end{array}$

$\begin{array}{lll}-1.08040200 & -2.29408900 & -0.93055600\end{array}$

$-2.46871000 \quad-2.38294100 \quad-0.76910800$

$\begin{array}{lll}-0.34740300 & -3.48710900 & -1.00655400\end{array}$

$\begin{array}{lll}-3.09561500 & -3.61866500 & -0.64725700\end{array}$

$\begin{array}{lll}-3.07563400 & -1.47718400 & -0.77568500\end{array}$

$\begin{array}{lll}-0.97485700 & -4.71915100 & -0.88845000\end{array}$

$\begin{array}{lll}0.73428100 & -3.42668100 & -1.13022800\end{array}$

$-2.35387600 \quad-4.79386000-0.70037500$

$\begin{array}{llll}-4.17952300 & -3.66122400 & -0.53507700\end{array}$

$\begin{array}{lll}-0.38038100 & -5.63128000 & -0.93971800\end{array}$

$\begin{array}{lll}-2.84580500 & -5.76084000 & -0.60766800\end{array}$

$\begin{array}{lll}-0.03552600 & 2.78268000 & -1.12839900\end{array}$

$\begin{array}{lll}-0.16100100 & 3.60262600 & -2.25866900\end{array}$

$\begin{array}{lll}0.05095600 & 3.40192000 & 0.12797700\end{array}$

$\begin{array}{llll}-0.20340700 & 4.98619800 & -2.14088100\end{array}$

$-0.24397500 \quad 3.13527100 \quad-3.24048400$

$\begin{array}{lll}-0.00557500 & 4.78545900 & 0.24557000\end{array}$

$\begin{array}{lll}0.21552700 & 2.78839000 & 1.01476900\end{array}$

$\begin{array}{lll}-0.13430400 & 5.58566600 & -0.88651100\end{array}$

$\begin{array}{lll}-0.30118200 & 5.60030800 & -3.03524200\end{array}$

$\begin{array}{llll}0.07088200 & 5.24361500 & 1.23104700\end{array}$
$\mathrm{H}$

$\mathrm{O}$

C

$\mathrm{H}$

$\mathrm{H}$

C

C

C

H

H

$\mathrm{H}$

N

C

C

$\mathrm{H}$

$\mathrm{H}$
$\mathrm{H}$

$\mathrm{H}$

B

$\mathrm{O}$

$\mathrm{O}$

\section{INT6}

Sum of electronic and zero-point Energies = $-2201.946299$

Sum of electronic and thermal Free Energies = $-2202.026153$

$\begin{array}{crrr}\text { Rh } & 1.56525200 & -0.10358000 & -0.11458800 \\ \mathrm{C} & 3.54716800 & 0.21829500 & -0.40068900 \\ \mathrm{C} & 4.18754700 & 0.94537900 & 0.62948300 \\ \mathrm{C} & 4.28442600 & -0.08204300 & -1.55165100 \\ \mathrm{C} & -0.76406900 & 0.25547100 & -0.48872800 \\ \mathrm{C} & -0.04573100 & 1.25709500 & -1.27784700 \\ \mathrm{C} & 0.74398900 & 0.53591600 & -2.18542700 \\ \mathrm{C} & 0.57826200 & -0.85917800 & -1.95186100 \\ \mathrm{C} & -0.46034000 & -1.04424900 & -0.98231100 \\ \mathrm{C} & -1.87223900 & 0.63089900 & 0.45200100 \\ \mathrm{C} & 5.51051900 & 1.37559500 & 0.48439000 \\ \mathrm{C} & 6.21633900 & 1.08511200 & -0.67310800 \\ \mathrm{C} & 5.59926700 & 0.35403000 & -1.68798500 \\ \mathrm{C} & 1.62668500 & 0.71463400 & -3.37264100 \\ \mathrm{H} & 3.81898500 & -0.64986400 & -2.35849800 \\ \mathrm{H} & 5.99359200 & 1.94769200 & 1.27724600 \\ \mathrm{H} & 6.15059800 & 0.11819600 & -2.59814900 \\ \mathrm{H} & 7.24490700 & 1.42401300 & -0.78511000 \\ \mathrm{H} & -1.64606300 & 1.60819600 & 0.90828100 \\ \mathrm{H} & 1.08737400 & 1.19806700 & -4.20687200 \\ \mathrm{H} & -2.79004700 & 0.80073700 & -0.13111500 \\ \mathrm{H} & 2.53887000 & 1.29916200 & -3.17661600 \\ \mathrm{C} & 1.23924900 & -1.60234100 & -3.06278800 \\ \mathrm{H} & 0.49499200 & -2.04584500 & -3.74963400 \\ \mathrm{H} & 1.92228100 & -2.39565100 & -2.73167100 \\ \mathrm{C} & -2.14008600 & -0.32838900 & 1.59176100 \\ \mathrm{~N} & -3.45074600 & -0.54244200 & 1.94991000 \\ \mathrm{H} & -3.52411300 & -1.26510300 & 2.65982300 \\ \mathrm{C} & -4.65194100 & -0.10329000 & 1.34140800 \\ \mathrm{C} & -5.59920200 & -1.03955800 & 0.93675900 \\ \mathrm{C} & -4.93897200 & 1.25743100 & 1.18722600 \\ \mathrm{C} & -6.81074600 & -0.64195300 & 0.37757200 \\ \mathrm{H} & -5.38006400 & -2.10019400 & 1.06235100 \\ \mathrm{C} & -6.12741400 & 1.66194100 & 0.60652700\end{array}$

$\begin{array}{rrr}-0.17347400 & 6.66960800 & -0.79198200 \\ -8.13580100 & 1.30706500 & -0.31286600 \\ -9.13780800 & 0.41896100 & -0.73984100 \\ -9.95531400 & 1.03607700 & -1.12116500 \\ -8.77880400 & -0.24036800 & -1.54473500 \\ -9.51126000 & -0.20011500 & 0.09026600 \\ 3.31285800 & 1.06370800 & 1.82791900 \\ 3.86536300 & 1.70122800 & 2.93322300 \\ 3.07828100 & 1.89994700 & 4.06093200 \\ 4.90717800 & 2.01442800 & 2.90918900 \\ 1.27804100 & 0.81085000 & 2.91564800 \\ 1.76748600 & 1.43863400 & 4.05463900 \\ 3.48997700 & 2.39356500 & 4.93929400 \\ 0.27085200 & 0.40014400 & 2.85491800 \\ 1.12443600 & 1.55056600 & 4.92431200 \\ 2.02720500 & 0.64876400 & 1.81950100 \\ 2.65128700 & -3.92798400 & 0.77197200 \\ 3.21839100 & -3.36970300 & 2.07396600 \\ 1.57023800 & -4.12582700 & 0.86126400 \\ 3.14930300 & -4.84625300 & 0.43490200 \\ 2.65106100 & -3.68679800 & 2.95804700 \\ 4.27237000 & -3.65994800 & 2.21833400 \\ 2.97951100 & -1.70293600 & 0.55914300 \\ 2.86252500 & -2.89202400 & -0.17394500 \\ 3.13301700 & -1.96845600 & 1.91966600 \\ -1.18346100 & -0.93206000 & 2.15317700\end{array}$

$\begin{array}{lll}-6.12741400 & 1.66194100 & 0.60652700\end{array}$ 


\begin{tabular}{|c|c|c|c|}
\hline & -4.22218100 & 1.99923800 & 1.53672600 \\
\hline & -7.07453700 & 0.71647600 & 0.19972700 \\
\hline $\mathrm{H}$ & -7.53054700 & -1.39890500 & 0.07682400 \\
\hline & -6.36067500 & 2.71602900 & 0.47222300 \\
\hline & 2.01126400 & -0.60766600 & -3.74020500 \\
\hline & -1.13176500 & -2.32170800 & -0.69897100 \\
\hline & -2.52876900 & -2.39637000 & -0.65833000 \\
\hline & -0.39871000 & -3.50718200 & -0.56429400 \\
\hline & -3.17204600 & -3.61380200 & -0.45943200 \\
\hline & -3.12459300 & -1.49571400 & -0.81373600 \\
\hline & -1.04248000 & -4.72143400 & -0.36713100 \\
\hline & 0.68912300 & -3.45761200 & -0.60612900 \\
\hline & -2.43273100 & -4.78175000 & -0.30771300 \\
\hline & -4.26175800 & -3.65024100 & -0.44829400 \\
\hline & -0.45193800 & -5.63045900 & -0.25635200 \\
\hline & -2.93589300 & -5.73523700 & -0.15463400 \\
\hline & -0.13352400 & 2.72079700 & -1.16670700 \\
\hline & -0.36488200 & 3.48785100 & -2.31505200 \\
\hline & 0.01942700 & 3.38912300 & 0.05587000 \\
\hline & -0.44620200 & 4.87384700 & -2.24588600 \\
\hline$\Pi$ & -0.49627400 & 2.97997800 & -3.27109600 \\
\hline & -0.07510300 & 4.77380200 & 0.12721400 \\
\hline & 0.25840300 & 2.81359100 & 0.95155700 \\
\hline & -0.30915200 & 5.52279900 & -1.02274600 \\
\hline 1 & -0.62534900 & 5.44957200 & -3.15291900 \\
\hline & 0.05410700 & 5.27288500 & 1.08672700 \\
\hline & -0.37606000 & 6.60804800 & -0.96656300 \\
\hline 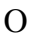 & -8.20956000 & 1.21599800 & -0.34396600 \\
\hline 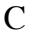 & -9.19058100 & 0.30400500 & -0.76999700 \\
\hline I & -10.01152100 & 0.90079000 & -1.17541500 \\
\hline & -8.80823400 & -0.36393200 & -1.55682000 \\
\hline 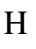 & -9.56728200 & -0.30564500 & 0.06552700 \\
\hline$c$ & 3.38701900 & 1.18714200 & 1.81995000 \\
\hline $\mathrm{C}$ & 3.83772600 & 1.80602000 & 2.98823100 \\
\hline & 2.98513700 & 1.93736900 & 4.07025600 \\
\hline 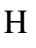 & 4.85958000 & 2.17431100 & 3.04202100 \\
\hline $\mathrm{C}$ & 1.29930300 & 0.81944200 & 2.79871600 \\
\hline C & 1.69263200 & 1.42770000 & 3.98031400 \\
\hline I & 3.32935900 & 2.42067100 & 4.98307200 \\
\hline & 0.31616900 & 0.36466900 & 2.67609500 \\
\hline & 0.99733300 & 1.48499300 & 4.81384700 \\
\hline 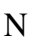 & 2.11376300 & 0.72545500 & 1.73861800 \\
\hline C & 3.41176100 & -3.86095600 & 0.33147700 \\
\hline 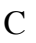 & 3.89835700 & -3.18366100 & 1.61143900 \\
\hline & 2.62057300 & -4.60222000 & 0.53349100 \\
\hline & 4.21167500 & -4.35473900 & -0.23293300 \\
\hline & 3.72743500 & -3.78492600 & 2.51244300 \\
\hline & 4.96876000 & -2.93105400 & 1.55968300 \\
\hline & 2.63279800 & -1.73468700 & 0.42497500 \\
\hline & 2.86437900 & -2.79820700 & -0.43718300 \\
\hline & 3.14261400 & -1.98439000 & 1.68193800 \\
\hline & & & \\
\hline
\end{tabular}

\section{$\operatorname{Ir}\left(\right.$ III) $(\text { dFppy })_{3}$}

Sum of electronic and zero-point Energies= $-2134.706560$

Sum of electronic and thermal Free Energies= $-2134.773401$

$\begin{array}{lrrr}\mathrm{C} & 2.54592400 & -1.42427000 & -0.44909200 \\ \mathrm{C} & 2.63891600 & -0.29382500 & 1.69326100 \\ \mathrm{C} & 3.85415400 & -1.87026700 & -0.22688100 \\ \mathrm{C} & 4.56591400 & -1.55822900 & 0.91517400 \\ \mathrm{C} & 3.93171300 & -0.76238300 & 1.86003400 \\ \mathrm{H} & 2.21086600 & 0.33201200 & 2.47821800 \\ \mathrm{H} & 5.57956400 & -1.91886900 & 1.05901000 \\ \mathrm{C} & 1.74459200 & -1.72152000 & -1.63240100\end{array}$

$2.16352900 \quad-2.44425400 \quad-2.75706100$

$\begin{array}{lll}1.30388900 & -2.63141700 & -3.82494700\end{array}$

$\begin{array}{lll}3.16884400 & -2.84544700 & -2.78395800\end{array}$

$-0.35128500 \quad-1.39771000 \quad-2.64199100$

$\begin{array}{lll}0.01880300 & -2.10013600 & -3.77344400\end{array}$

$\begin{array}{lll}1.63889100 & -3.18822500 & -4.69810500\end{array}$

$\begin{array}{lll}-1.33622700 & -0.94579200 & -2.54275700\end{array}$

$-0.68665400 \quad-2.21958300 \quad-4.59108600$

$\begin{array}{llll}0.47790400 & -1.20969200 & -1.60184200\end{array}$

$\begin{array}{lll}1.92087500 & -0.61450200 & 0.53810100\end{array}$

$\begin{array}{llll}0.59760600 & 1.64134100 & -0.67123600\end{array}$

$\begin{array}{lll}0.53282600 & 2.73484800 & 0.23882400\end{array}$

$\begin{array}{lll}1.05804600 & 1.87773000 & -1.97162300\end{array}$

$\begin{array}{llll}0.92527000 & 4.00343300 & -0.20277400\end{array}$

$\begin{array}{llll}1.43075100 & 3.15559200 & -2.34727600\end{array}$

$\begin{array}{lll}1.13456700 & 1.07979700 & -2.70935500\end{array}$

$\begin{array}{llll}1.37453500 & 4.24414700 & -1.48466000\end{array}$

$\begin{array}{lll}1.67080000 & 5.23947500 & -1.79968700\end{array}$

$\begin{array}{lll}0.04315600 & 2.41550500 & 1.56818500\end{array}$

$\begin{array}{lll}-0.08034900 & 3.28967600 & 2.65625700\end{array}$

$\begin{array}{lll}-0.55068900 & 2.82418200 & 3.87049900\end{array}$

$\begin{array}{lll}0.20085800 & 4.32850000 & 2.53451400\end{array}$

$\begin{array}{llll}-0.76848600 & 0.66125400 & 2.90152300\end{array}$

$\begin{array}{llll}-0.90392500 & 1.48297800 & 4.00329500\end{array}$

$\begin{array}{lll}-0.64148600 & 3.50615000 & 4.71370500\end{array}$

$\begin{array}{lll}-1.02618800 & -0.39553800 & 2.93866100\end{array}$

$\begin{array}{lll}-1.27849900 & 1.07769400 & 4.93922400\end{array}$

$\begin{array}{lll}-0.30792800 & 1.10477300 & 1.72107000\end{array}$

$\begin{array}{lll}-0.03560500 & -0.09947100 & 0.07572900\end{array}$

$\begin{array}{llll}-2.05866400 & 0.14545100 & -0.40907200\end{array}$

$\begin{array}{lll}-2.91652200 & -0.90925800 & 0.01777000\end{array}$

$\begin{array}{lll}-2.62329100 & 1.22813500 & -1.09105800\end{array}$

$\begin{array}{lll}-4.28741600 & -0.81098100 & -0.25816900\end{array}$

$\begin{array}{llll}-3.98537400 & 1.26839700 & -1.33768400\end{array}$

$\begin{array}{lll}-2.00830100 & 2.05779200 & -1.44182400\end{array}$

$\begin{array}{llll}-4.84544400 & 0.25776100 & -0.93119100\end{array}$

$\begin{array}{llll}-5.91245400 & 0.29985700 & -1.12618200\end{array}$

$\begin{array}{lll}-2.29221000 & -2.03497900 & 0.71904800\end{array}$

$-2.94736600 \quad-3.18213200 \quad 1.19216100$

$\begin{array}{lll}-0.26268200 & -2.87501800 & 1.53843200\end{array}$

$\begin{array}{lll}-2.23014600 & -4.17081300 & 1.84403200\end{array}$

$\begin{array}{lll}-4.01420300 & -3.28972200 & 1.04424600\end{array}$

$\begin{array}{lll}-0.85853200 & -4.02404700 & 2.02784200\end{array}$

$\begin{array}{lll}0.80657100 & -2.69172200 & 1.64766400\end{array}$

$\begin{array}{lll}-2.74505100 & -5.05798800 & 2.20881200\end{array}$

$\begin{array}{lll}-0.26139000 & -4.77732300 & 2.53469800\end{array}$

$\begin{array}{lll}-0.95009100 & -1.91288900 & 0.90887600\end{array}$

$\begin{array}{lll}-4.50644900 & 2.30941900 & -1.99142700\end{array}$

$\begin{array}{lll}-5.14149500 & -1.77664300 & 0.12854000\end{array}$

$\begin{array}{lll}4.48147700 & -2.64430000 & -1.12984400\end{array}$

$\begin{array}{lll}4.60625100 & -0.44950300 & 2.96947000\end{array}$

$\begin{array}{lll}1.86455100 & 3.36722900 & -3.59203900\end{array}$ $\begin{array}{llll}0.87046300 & 5.05654500 & 0.62897800\end{array}$

\section{$\operatorname{Ir}($ II $)(\text { dFppy })_{3}$}

Sum of electronic and zero-point Energies= -2134.729855

Sum of electronic and thermal Free Energies= -2134.797806

$\begin{array}{lllc}\mathrm{C} & 2.50996500 & -1.41937400 & -0.65505500 \\ \mathrm{C} & 2.61205400 & -0.57411700 & 1.62528200 \\ \mathrm{C} & 3.79335800 & -1.94892800 & -0.45930200 \\ \mathrm{C} & 4.49636600 & -1.81271700 & 0.72279000 \\ \mathrm{C} & 3.88265800 & -1.11119700 & 1.75294900 \\ \mathrm{H} & 2.18703800 & -0.03868700 & 2.47727700 \\ \mathrm{H} & 5.48905000 & -2.23956600 & 0.83240100\end{array}$




\begin{tabular}{|c|c|c|c|}
\hline C & 1.72526000 & -1.51522700 & -1.86952700 \\
\hline & 2.15107900 & -2.03342700 & -3.10187800 \\
\hline 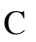 & 1.30762100 & -2.05433400 & -4.19493300 \\
\hline$\theta$ & 3.16335300 & -2.41398400 & -3.18255700 \\
\hline$C^{2}$ & -0.37554200 & -1.03524500 & -2.83475300 \\
\hline & -0.00024500 & -1.54929400 & -4.05481200 \\
\hline $\mathrm{H}$ & 1.65512400 & -2.44993200 & -5.14811100 \\
\hline $\mathrm{H}$ & -1.36593700 & -0.61146900 & -2.67423500 \\
\hline H & -0.71027600 & -1.55394200 & -4.87840800 \\
\hline $\mathrm{N}$ & 0.44100200 & -0.99881000 & -1.76198800 \\
\hline 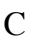 & 1.90050400 & -0.72129800 & 0.43091800 \\
\hline $\mathrm{C}$ & 0.60731200 & 1.69696700 & -0.50761800 \\
\hline 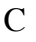 & 0.59483800 & 2.67390800 & 0.53605100 \\
\hline $\mathrm{C}$ & 1.05190100 & 2.08464200 & -1.78119700 \\
\hline $\mathrm{C}$ & 1.01329700 & 3.97610100 & 0.23920100 \\
\hline $\mathrm{C}$ & 1.44907400 & 3.38761100 & -2.00625200 \\
\hline H & 1.09200200 & 1.37434900 & -2.60701800 \\
\hline C & 1.43974800 & 4.36379900 & -1.01411500 \\
\hline H & 1.75417300 & 5.38405600 & -1.21066400 \\
\hline$y$ & 0.13028200 & 2.20482700 & 1.82561400 \\
\hline C & 0.07305300 & 2.93470800 & 3.02247700 \\
\hline C & -0.39593100 & 2.34479200 & 4.18023200 \\
\hline $\mathrm{H}$ & 0.40487900 & 3.96629500 & 3.02393500 \\
\hline 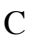 & -0.74110000 & 0.33117100 & 2.94834800 \\
\hline C & -0.82182900 & 1.01223400 & 4.14612100 \\
\hline $\mathrm{H}$ & -0.43205900 & 2.91636100 & 5.10649600 \\
\hline H & -1.04914200 & -0.70993400 & 2.86042700 \\
\hline H & -1.20643600 & 0.50819700 & 5.02919600 \\
\hline N & -0.27177300 & 0.89131500 & 1.82080700 \\
\hline$[\mathrm{r}$ & -0.04361000 & -0.10873300 & 0.03622900 \\
\hline $\mathrm{C}$ & -2.06693200 & 0.19586500 & -0.41925000 \\
\hline $\mathrm{C}$ & -2.93602300 & -0.87962800 & -0.05166400 \\
\hline $\mathrm{C}$ & -2.61823000 & 1.31393300 & -1.05660700 \\
\hline $\mathrm{C}$ & -4.29586200 & -0.76669100 & -0.37810000 \\
\hline $\mathrm{C}$ & -3.97297300 & 1.37121300 & -1.33571400 \\
\hline $\mathrm{H}$ & -1.98858900 & 2.15461100 & -1.35342100 \\
\hline $\mathrm{C}$ & -4.83834600 & 0.33411000 & -1.01227500 \\
\hline $\mathrm{H}$ & -5.89819400 & 0.37613200 & -1.24602300 \\
\hline $\mathrm{C}$ & -2.33588500 & -2.00983000 & 0.64114300 \\
\hline $\mathrm{C}$ & -3.02106900 & -3.07844500 & 1.24248800 \\
\hline $\mathrm{C}$ & -0.29952600 & -2.94730300 & 1.36045200 \\
\hline $\mathrm{C}$ & -2.33127200 & -4.08229100 & 1.89575200 \\
\hline $\mathrm{H}$ & -4.10381800 & -3.10396300 & 1.19337500 \\
\hline $\mathrm{C}$ & -0.92522100 & -4.02530100 & 1.94879300 \\
\hline $\mathrm{H}$ & 0.78584300 & -2.83563100 & 1.38464700 \\
\hline $\mathrm{H}$ & -2.87452700 & -4.90150200 & 2.36509000 \\
\hline $\mathrm{H}$ & -0.33520200 & -4.80140100 & 2.43092300 \\
\hline $\mathrm{N}$ & -0.95892700 & -1.95751400 & 0.73281200 \\
\hline 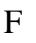 & -4.48847100 & 2.45680700 & -1.94408200 \\
\hline $\mathrm{F}$ & -5.16247100 & -1.76788400 & -0.09258200 \\
\hline F & 4.40988100 & -2.64785400 & -1.44121200 \\
\hline $\mathrm{F}$ & 4.56059700 & -0.96242300 & 2.90829700 \\
\hline F & 1.86574100 & 3.75072400 & -3.23117500 \\
\hline & 1.00853100 & 4.93064300 & 1.19544900 \\
\hline
\end{tabular}

\section{${ }^{3} \operatorname{Ir}(\mathbf{I I I})(\mathbf{d F p p y})_{3}$ *}

\begin{tabular}{|c|c|c|c|}
\hline & correction $=$ & \multicolumn{2}{|c|}{0.427168} \\
\hline & rrection to $\mathrm{Gib}$ & bs Free Energy & 0.360343 \\
\hline $\mathrm{C}$ & -2.69252500 & -1.02167600 & 0.46020700 \\
\hline $\mathrm{C}$ & -2.53169800 & -0.07337100 & -1.78157800 \\
\hline $\mathrm{C}$ & -4.03751200 & -1.28206800 & 0.19674400 \\
\hline $\mathrm{C}$ & -4.64750400 & -0.96700800 & -1.01151500 \\
\hline $\mathrm{C}$ & -3.88050700 & -0.36254600 & -1.98467400 \\
\hline $\mathrm{H}$ & -1.96998900 & 0.40339100 & -2.58418400 \\
\hline $\mathrm{H}$ & -5.69754600 & -1.19057400 & -1.17357900 \\
\hline
\end{tabular}

\begin{tabular}{|c|c|c|c|}
\hline $\mathrm{C}$ & -1.98368200 & -1.34039000 & 1.70120600 \\
\hline $\mathrm{C}$ & -2.51956100 & -1.96423400 & 2.82937800 \\
\hline $\mathrm{C}$ & -1.71987500 & -2.21870400 & 3.93220500 \\
\hline $\mathrm{H}$ & -3.56389900 & -2.25180400 & 2.83161600 \\
\hline $\mathrm{C}$ & 0.11614500 & -1.23484800 & 2.76905000 \\
\hline $\mathrm{C}$ & -0.37148200 & -1.85107900 & 3.90375000 \\
\hline $\mathrm{H}$ & -2.14228800 & -2.70576300 & 4.80808400 \\
\hline $\mathrm{H}$ & 1.15289900 & -0.91401400 & 2.67876900 \\
\hline $\mathrm{H}$ & 0.29086800 & -2.03656100 & 4.74456600 \\
\hline $\mathrm{N}$ & -0.66081500 & -0.97366400 & 1.70397800 \\
\hline $\mathrm{C}$ & -1.95491400 & -0.39378600 & -0.56496000 \\
\hline $\mathrm{C}$ & -0.30803300 & 1.85688200 & 0.62752900 \\
\hline $\mathrm{C}$ & 0.00186800 & 2.85176300 & -0.34400500 \\
\hline $\mathrm{C}$ & -0.74650900 & 2.25482800 & 1.89723400 \\
\hline $\mathrm{C}$ & -0.14133800 & 4.18980100 & 0.00749100 \\
\hline $\mathrm{C}$ & -0.86831800 & 3.60284700 & 2.18794700 \\
\hline $\mathrm{H}$ & -0.99369400 & 1.52087400 & 2.66254100 \\
\hline $\mathrm{C}$ & -0.57229100 & 4.59002300 & 1.26144400 \\
\hline $\mathrm{H}$ & -0.67163000 & 5.64384600 & 1.50495700 \\
\hline $\mathrm{C}$ & 0.45615600 & 2.35714300 & -1.63830900 \\
\hline $\mathrm{C}$ & 0.77619100 & 3.11816200 & -2.76398600 \\
\hline $\mathrm{C}$ & 1.16778900 & 2.48342100 & -3.93062100 \\
\hline $\mathrm{H}$ & 0.70965200 & 4.19892500 & -2.71587800 \\
\hline $\mathrm{C}$ & 0.91952000 & 0.39226100 & -2.82292200 \\
\hline $\mathrm{C}$ & 1.24058800 & 1.10232500 & -3.97010400 \\
\hline $\mathrm{H}$ & 1.41577600 & 3.07202700 & -4.81110200 \\
\hline $\mathrm{H}$ & 0.95765400 & -0.69484900 & -2.76823900 \\
\hline $\mathrm{H}$ & 1.54574800 & 0.57262100 & -4.86777900 \\
\hline $\mathrm{N}$ & 0.53913500 & 1.00363500 & -1.69942600 \\
\hline $\mathrm{Ir}$ & 0.00229800 & -0.01404800 & 0.01486800 \\
\hline $\mathrm{C}$ & 2.05297100 & -0.28244800 & 0.36982800 \\
\hline $\mathrm{C}$ & 2.63421100 & -1.51632300 & -0.02186400 \\
\hline $\mathrm{C}$ & 2.78838400 & 0.73198300 & 0.97345200 \\
\hline $\mathrm{C}$ & 4.01483300 & -1.64445000 & 0.24677300 \\
\hline $\mathrm{C}$ & 4.14479800 & 0.52370900 & 1.21350600 \\
\hline $\mathrm{H}$ & 2.35305900 & 1.68096400 & 1.27988300 \\
\hline $\mathrm{C}$ & 4.77130200 & -0.66197900 & 0.85247900 \\
\hline $\mathrm{H}$ & 5.83063500 & -0.82092500 & 1.03249600 \\
\hline $\mathrm{C}$ & 1.77358100 & -2.48139400 & -0.63140400 \\
\hline $\mathrm{C}$ & 2.12898800 & -3.77407000 & -1.05822300 \\
\hline $\mathrm{C}$ & -0.43982900 & -2.88338300 & -1.37028300 \\
\hline $\mathrm{C}$ & 1.21598500 & -4.61756300 & -1.64026800 \\
\hline $\mathrm{H}$ & 3.15401200 & -4.10070700 & -0.91668100 \\
\hline $\mathrm{C}$ & -0.12594900 & -4.14196900 & -1.80523200 \\
\hline $\mathrm{H}$ & -1.45643500 & -2.51292800 & -1.48711700 \\
\hline $\mathrm{H}$ & 1.50641300 & -5.61339200 & -1.96392800 \\
\hline $\mathrm{H}$ & -0.89598900 & -4.75669300 & -2.26416800 \\
\hline $\mathrm{N}$ & 0.43992500 & -2.02455500 & -0.79279000 \\
\hline $\mathrm{F}$ & 4.86093200 & 1.49281000 & 1.80882900 \\
\hline $\mathrm{F}$ & 4.65893200 & -2.77520000 & -0.09857400 \\
\hline $\mathrm{F}$ & -4.81438000 & -1.85994000 & 1.12198000 \\
\hline $\mathrm{F}$ & -4.44420000 & -0.05559200 & -3.15197300 \\
\hline $\mathrm{F}$ & -1.28308100 & 3.98134400 & 3.39211800 \\
\hline$\Gamma$ & 0.14395300 & 5.16082100 & -0.86783400 \\
\hline
\end{tabular}

\section{INT2-Rh(II)}

Sum of electronic and zero-point Energies= $-1948.731745$

Sum of electronic and thermal Free Energies= $-1948.807632$

$\begin{array}{crrr}\text { Rh } & 2.23541800 & 0.21067800 & -0.28230100 \\ \text { C } & 2.93531700 & -0.29096600 & 2.54279000 \\ \text { C } & 2.13288900 & 1.94997600 & 2.19460300 \\ \text { C } & -0.39784400 & 0.62672000 & -1.08722700 \\ \text { C } & 0.52791400 & 1.66622300 & -1.41921100 \\ \text { C } & 1.53650000 & 1.05782500 & -2.23487700\end{array}$




$\begin{array}{lrrr}\mathrm{H} & 3.07097400 & -2.86454700 & -1.05295000 \\ \mathrm{H} & 4.30566200 & -4.75428900 & 0.02679800 \\ \mathrm{~N} & 2.98319900 & -1.71773300 & 0.64832500 \\ \mathrm{O} & -0.30716900 & -0.92801200 & 1.35954200 \\ \mathrm{C} & 2.50497400 & 0.71439100 & 1.63955200 \\ \mathrm{H} & 3.67421000 & 0.88134700 & -0.42564000\end{array}$

\section{${ }^{3}$ INT2*}

$\mathrm{E}(\mathrm{TD}-\mathrm{DFT})=-1949.253836$

Zero-point correction $=\quad 0.616870$

Thermal correction to Gibbs Free Energy $=0.544982$

Rh $\quad 231898600 \quad 0.45741100 \quad-0.26245100$

$\begin{array}{llll}\text { C } & 2.41824200 & -0.09505200 & 2.72064900\end{array}$

$\begin{array}{llll}\text { C } & 2.16914700 & 2.24758600 & 2.14594400\end{array}$

C $\quad-0.08611000 \quad 0.39395900 \quad-1.05184300$

$\begin{array}{lllll}\mathrm{C} & 0.61023700 & 1.58085000 & -1.48464600\end{array}$

C $\quad 1.65323000 \quad 1.12010300 \quad-2.34151600$

C $\quad 1.55593100 \quad-0.28680300 \quad-2.46120400$

C $\quad 0.40874000 \quad-0.75012700 \quad-1.76362400$

$\begin{array}{llll}\text { C } & -1.17455800 & 0.43009100 & -0.02604100\end{array}$

$\begin{array}{llll}\text { C } & 2.33484600 & 0.26089200 & 4.06961400\end{array}$

$\begin{array}{llll}\mathrm{C} & 2.15770800 & 1.58356800 & 4.45612000\end{array}$

$\begin{array}{llll}\mathrm{C} & 2.05586500 & 2.58082100 & 3.49122800\end{array}$

$\begin{array}{llll}\mathrm{C} & 2.59307800 & 1.61746300 & -3.39241100\end{array}$

$\mathrm{H} \quad 2.11428200 \quad 3.04311800 \quad 1.39955700$

$\mathrm{H} \quad 2.38316700 \quad-0.50588100 \quad 4.84244800$

$\begin{array}{llll}\mathrm{H} & 1.91644600 & 3.62003400 & 3.78844200\end{array}$

$\begin{array}{llll}\mathrm{H} & 2.09323300 & 1.83378600 & 5.51379900\end{array}$

$\mathrm{H} \quad-1.06601900 \quad 1.35942700 \quad 0.55708900$

$\begin{array}{llll}\mathrm{H} & 2.03903700 & 2.13197400 & -4.20031900\end{array}$

$\mathrm{H} \quad-2.16551500 \quad 0.50115700 \quad-0.50048100$

$\mathrm{H} \quad 3.37993400 \quad 2.29697000 \quad-3.04002900$

C $\quad 2.46641000 \quad-0.71894800 \quad-3.56576900$

$\mathrm{H} \quad \begin{array}{llll}\mathrm{H} & 1.88453600 & -1.06548600 & -4.44196100\end{array}$

$\mathrm{H} \quad 3.17671600-1.51759300 \quad-3.30469200$

$\begin{array}{llll}\mathrm{C} & -1.16592300 & -0.69870200 & 0.98939000\end{array}$

$\mathrm{N} \quad-2.38539500 \quad-1.02028500 \quad 1.54883500$

$\begin{array}{llll}\mathrm{H} & -2.31589100 & -1.82207400 & 2.16748700\end{array}$

$\begin{array}{llll}\mathrm{C} & -3.67931300 & -0.55164400 & 1.21100000\end{array}$

$\begin{array}{lllll}\text { C } & -4.64118500 & -1.44731100 & 0.75255000\end{array}$

$\begin{array}{llll}\text { C } & -4.02851200 & 0.79559400 & 1.35995900\end{array}$

$\begin{array}{llll}\text { C } & -5.93037100 & -1.02309100 & 0.44123000\end{array}$

$\begin{array}{llll}\mathrm{H} & -4.37207000 & -2.49752000 & 0.63731500\end{array}$

$\begin{array}{llll}\mathrm{C} & -5.29739000 & 1.23274600 & 1.02583200\end{array}$

$\begin{array}{llll}\mathrm{H} & -3.29206100 & 1.49813200 & 1.74838100\end{array}$

$\begin{array}{llll}\text { C } & -6.25997900 & 0.32661700 & 0.56773100\end{array}$

$\mathrm{H} \quad-6.65952200 \quad-1.74915500 \quad 0.09081700$

$\mathrm{H} \quad-\quad-5.58212900 \quad 2.27771100 \quad 1.12967600$

$\begin{array}{lllll}\mathrm{O} & 3.22522800 & 0.43990700 & -3.88073300\end{array}$

C $\quad-0.13174200 \quad-2.10269800 \quad-1.83385200$

C $\quad-1.48266600 \quad-2.38256500 \quad-1.57083500$

$\begin{array}{llll}\text { C } & 0.66941200 & -3.16863400 & -2.27324100\end{array}$

C $\quad-1.99053200 \quad-3.66822800 \quad-1.69854100$

$\mathrm{H} \quad-2.16129600 \quad-1.57632400 \quad-1.29686400$

C $\quad 0.16295800 \quad-4.45462500 \quad-2.40176000$

$\mathrm{H} \quad 1.71372100 \quad-2.97845800 \quad-2.51780800$

C $\quad-1.17119800 \quad-4.71677100 \quad-2.10623200$

$\mathrm{H} \quad-3.04579900 \quad-3.84916000 \quad-1.49544000$

$\mathrm{H} \quad 0.81590700 \quad-5.25840300 \quad-2.73974900$

$\mathrm{H} \quad-1.57150900 \quad-5.72411800 \quad-2.20706300$

C $\quad 0.34031000 \quad 2.98877200 \quad-1.14624100$

C $\quad 1.39025200 \quad 3.91387000 \quad-1.02243100$

$\begin{array}{lllll}\text { C } & -0.96846300 & 3.46589300 & -0.98950500\end{array}$

C $\quad 1.14130500 \quad 5.24953700 \quad-0.73641200$

$\mathrm{H} \quad 2.41745900 \quad 3.56472000 \quad-1.12475700$

C $\quad-1.21671900 \quad 4.80314800 \quad-0.69530400$ 


$\begin{array}{lrrr}\mathrm{H} & -1.80852400 & 2.78625900 & -1.13053000 \\ \mathrm{C} & -0.16487800 & 5.70136100 & -0.56299700 \\ \mathrm{H} & 1.97591800 & 5.94205500 & -0.63707400 \\ \mathrm{H} & -2.24426000 & 5.14607700 & -0.58126600 \\ \mathrm{H} & -0.36006800 & 6.74722600 & -0.33174300 \\ \mathrm{O} & -7.47407500 & 0.85265500 & 0.27989200 \\ \mathrm{C} & -8.47409900 & -0.01892300 & -0.18491800 \\ \mathrm{H} & -9.36211500 & 0.59429800 & -0.35757100 \\ \mathrm{H} & -8.18229200 & -0.50354600 & -1.12906400 \\ \mathrm{H} & -8.71201300 & -0.79566800 & 0.55778500 \\ \mathrm{C} & 2.53878800 & -1.51413600 & 2.33095600 \\ \mathrm{C} & 2.70857300 & -2.55337900 & 3.25182000 \\ \mathrm{C} & 2.81904600 & -3.86093200 & 2.80427600 \\ \mathrm{H} & 2.76041200 & -2.33997500 & 4.31672400 \\ \mathrm{C} & 2.59571500 & -3.04176200 & 0.58802900 \\ \mathrm{C} & 2.77084400 & -4.12332300 & 1.44052600 \\ \mathrm{H} & 2.95079500 & -4.67169300 & 3.51897700 \\ \mathrm{H} & 2.55950300 & -3.17617300 & -0.49421100 \\ \mathrm{H} & 2.86313000 & -5.13198800 & 1.04585300 \\ \mathrm{~N} & 2.46285100 & -1.79461800 & 1.02009100 \\ \mathrm{O} & -0.15954300 & -1.27832900 & 1.34744400 \\ \mathrm{C} & 2.35003700 & 0.92066100 & 1.73307400 \\ \mathrm{H} & 3.94524300 & 0.61193900 & -0.35237000\end{array}$

\section{${ }^{3}$ INT3*}

$\mathrm{E}(\mathrm{TD}-\mathrm{DFT})=-2456.994994$

Zero-point correction= 0.758320

Thermal correction to Gibbs Free Energy $=0.666491$

$\begin{array}{llll}\mathrm{Rh} & 1.07153300 & -0.24730500 & -0.14186600\end{array}$

$\begin{array}{llll}\text { C } & 1.48743400 & 0.01948900 & 2.73801500\end{array}$

$\begin{array}{llll}\text { C } & 0.42112000 & 2.00024000 & 1.83691300\end{array}$

$\begin{array}{llll}\text { C } & -1.11124600 & 0.00673500 & -0.99563400\end{array}$

$\begin{array}{lllll}\text { C } & -0.14986900 & 0.89730700 & -1.62693400\end{array}$

$\begin{array}{llll}\text { C } & 0.72238800 & 0.05892100 & -2.37495700\end{array}$

C $\quad 0.36285800 \quad-1.28314300 \quad-2.15971100$

C $\quad-\quad \begin{array}{llll}\text { C } & -0.81030900 & -1.33991600 & -1.35276300\end{array}$

$\begin{array}{llll}\text { C } & -2.19558700 & 0.43977500 & -0.05992000\end{array}$

$\begin{array}{llll}\text { C } & 1.38404400 & 0.55891900 & 4.02348900\end{array}$

$\begin{array}{llll}\text { C } & 0.80140800 & 1.80375300 & 4.21705100\end{array}$

$\begin{array}{llll}\text { C } & 0.32652500 & 2.52701100 & 3.11942500\end{array}$

C $\quad 1.79475100 \quad 0.10413300 \quad-3.41426100$

$\begin{array}{llll}\mathrm{H} & 0.06527900 & 2.59578400 & 0.99257200\end{array}$

$\begin{array}{llll}\mathrm{H} & 1.75690900 & 0.00411500 & 4.88462600\end{array}$

$\mathrm{H} \quad-0.11825600 \quad 3.51102400 \quad 3.26787400$

$\begin{array}{llll}\mathrm{H} & 0.72025700 & 2.21579900 & 5.22140700\end{array}$

$\begin{array}{lllll}\mathrm{H} & -1.93714100 & 1.42965800 & 0.35190400\end{array}$

$\mathrm{H} \quad \begin{array}{llll}\mathrm{H} & 1.39139500 & 0.47400200 & -4.37514900\end{array}$

$\mathrm{H} \quad-3.14575900 \quad 0.58272400 \quad-0.59555900$

$\begin{array}{llll}\mathrm{H} & 2.68900800 & 0.69336000 & -3.16402700\end{array}$

C $\quad 1.18376800 \quad-2.13871300 \quad-3.06460300$

$\mathrm{H} \quad 0.58017100 \quad-2.53916100 \quad-3.90100500$

$\mathrm{H} \quad \begin{array}{llll}\mathrm{H} & 1.68037800 & -2.99420500 & -2.58137400\end{array}$

$\begin{array}{llll}\mathrm{C} & -2.39437100 & -0.48979100 & 1.12847100\end{array}$

N $\quad \begin{array}{llll}\mathrm{N} & -3.67791000 & -0.59861600 & 1.61727000\end{array}$

$\begin{array}{llll}\mathrm{H} & -3.74229000 & -1.29860100 & 2.34974100\end{array}$

$\begin{array}{llll}\mathrm{C} & -4.88293500 & -0.04330700 & 1.12181700\end{array}$

$\begin{array}{llll}\text { C } & -5.91855900 & -0.88218900 & 0.72065700\end{array}$

$\begin{array}{llll}\mathrm{C} & -5.07482600 & 1.34156600 & 1.06514500\end{array}$

$\begin{array}{llll}\text { C } & -7.12901100 & -0.36401200 & 0.26725200\end{array}$

$\begin{array}{llll}\mathrm{H} & -5.76881600 & -1.96121500 & 0.76257200\end{array}$

$\begin{array}{llll}\text { C } & -6.26226200 & 1.86692100 & 0.58874600\end{array}$

$\begin{array}{llll}\mathrm{H} & -4.28048900 & 2.00349700 & 1.40813700\end{array}$

$\begin{array}{lllll}\mathrm{C} & -7.30090300 & 1.01839100 & 0.19097900\end{array}$

$\mathrm{H} \quad-7.92078000 \quad-1.04578300 \quad-0.03285100$

$\mathrm{H} \quad-6.42468900 \quad 2.94132100 \quad 0.53284400$

O $\quad 2.19748100-1.26254400 \quad-3.54596000$
$-1.56064700 \quad-2.55232600-1.00624500$

$-2.95608200 \quad-2.58399700-1.11295700$

$\begin{array}{lll}-0.89929400 & -3.71702600 & -0.60044400\end{array}$

$\begin{array}{lll}-3.66780800 & -3.74081000 & -0.81115000\end{array}$

$-3.48766100-1.69689300-1.46064600$

$\begin{array}{lll}-1.61029500 & -4.87188900 & -0.29955500\end{array}$

$\begin{array}{lll}0.18529800 & -3.69760700 & -0.48685100\end{array}$

$\begin{array}{lll}-2.99855800 & -4.88913500 & -0.40208400\end{array}$

$\begin{array}{lll}-4.75286400 & -3.74845500 & -0.91569900\end{array}$

$\begin{array}{lll}-1.07679000 & -5.76387800 & 0.02630100\end{array}$

$\begin{array}{lll}-3.55480000 & -5.79532200 & -0.16732200\end{array}$

$\begin{array}{llll}-0.15896800 & 2.35967700 & -1.67182700\end{array}$

$\begin{array}{lll}1.05401500 & 3.06710800 & -1.60139500\end{array}$

$\begin{array}{llll}-1.35287500 & 3.08407400 & -1.79587600\end{array}$

$\begin{array}{llll}1.06351800 & 4.45467400 & -1.64155300\end{array}$

$\begin{array}{lll}1.99156000 & 2.52347800 & -1.46404800\end{array}$

$\begin{array}{llll}-1.33835900 & 4.47335400 & -1.82658000\end{array}$

$\begin{array}{lll}-2.29889400 & 2.55075500 & -1.88675100\end{array}$

$-0.13097500 \quad 5.16457800 \quad-1.74774300$

$\begin{array}{llll}2.01498100 & 4.98130700 & -1.57610700\end{array}$

$-2.27618900 \quad 5.01904000 \quad-1.92116800$

$\begin{array}{lll}-0.12156500 & 6.25319800 & -1.76992400\end{array}$

$\begin{array}{llll}-8.42761300 & 1.63116900 & -0.24542600\end{array}$

$\begin{array}{llll}-9.50169900 & 0.81993800 & -0.64927400\end{array}$

$\begin{array}{llll}-10.30219000 & 1.49770100 & -0.95651300\end{array}$

$\begin{array}{lll}-9.22749700 & 0.17799800 & -1.50032300\end{array}$

$\begin{array}{lll}-9.86318100 & 0.18621100 & 0.17499900\end{array}$

$\begin{array}{lll}2.11833100 & -1.27114800 & 2.46646500\end{array}$

$\begin{array}{lll}2.75853000 & -2.08374700 & 3.40835100\end{array}$

$\begin{array}{lll}3.35490700 & -3.26460000 & 3.00383000\end{array}$

$\begin{array}{lll}2.79314800 & -1.77864600 & 4.45191500\end{array}$

$\begin{array}{lll}2.67751500 & -2.77331000 & 0.77746900\end{array}$

$3.32411100 \quad-3.62369500 \quad 1.65584900$

$\begin{array}{lll}3.85508700 & -3.89993700 & 3.73238100\end{array}$

$\begin{array}{lll}2.63113100 & -2.96817300 & -0.29697000\end{array}$

$\begin{array}{lll}3.79782400 & -4.53304300 & 1.29425000\end{array}$

$\begin{array}{lll}2.07850300 & -1.64666300 & 1.16859300\end{array}$

$-1.48624800 \quad-1.10186200 \quad 1.65914800$

$\begin{array}{lll}0.98183400 & 0.73541200 & 1.62018100\end{array}$

$\begin{array}{lll}2.48629200 & 0.57032500 & -0.16291800\end{array}$

$\begin{array}{lll}4.15990100 & 2.54439800 & 2.32902700\end{array}$

$\begin{array}{lll}3.71855600 & 3.45935800 & 1.17633600\end{array}$

$\begin{array}{lll}3.35937900 & 2.37178400 & 3.06126400\end{array}$

$\begin{array}{lll}5.04160900 & 2.93191100 & 2.85995400\end{array}$

$\begin{array}{lll}2.62780100 & 3.61159300 & 1.16651000\end{array}$

$\begin{array}{lll}4.20794500 & 4.44203400 & 1.19510900\end{array}$

$\begin{array}{lll}5.20510600 & -0.62967200 & -2.81523300\end{array}$

$\begin{array}{lll}5.03366500 & -1.73024500 & -1.76836900\end{array}$

$\begin{array}{lll}4.58380600 & -0.79701200 & -3.70214900\end{array}$

$\begin{array}{lll}6.25358500 & -0.50692700 & -3.12551600\end{array}$

$\begin{array}{lll}4.07934100 & -2.26861900 & -1.90500500\end{array}$

$\begin{array}{lll}5.84844600 & -2.46470200 & -1.76941700\end{array}$

$\begin{array}{lll}4.44969400 & 1.48731700 & 0.35084000\end{array}$

$\begin{array}{lll}4.75928200 & 0.28870900 & -0.79865600\end{array}$

$\begin{array}{lll}4.50372500 & 1.30982300 & 1.71088500\end{array}$

$\begin{array}{lll}4.09291400 & 2.76919200 & -0.00841400\end{array}$

$\begin{array}{llll}4.78946100 & 0.56193500 & -2.14982000\end{array}$

$\begin{array}{lll}5.00754600 & -1.03811800 & -0.52459800\end{array}$

\section{INT4*}

$\mathrm{E}(\mathrm{TD}-\mathrm{DFT})=-2135.037083$

Zero-point correction= 0.760121

Thermal correction to Gibbs Free Energy= 0.672695

Rh $\quad 1.11608800 \quad 0.28262200 \quad-0.31963900$

$\begin{array}{llll}\text { C } & 1.84791500 & -0.86636900 & 2.36816800\end{array}$

C $\quad 1.96151000 \quad 1.54492000 \quad 2.27082200$ 


$\begin{array}{lrrr}\mathrm{C} & 1.56321500 & -3.15224600 & -0.43056000 \\ \mathrm{C} & 1.66631200 & -4.43067500 & 0.09985100 \\ \mathrm{H} & 1.89420300 & -5.52344500 & 1.94810300 \\ \mathrm{H} & 1.45258000 & -2.99643700 & -1.50394100 \\ \mathrm{H} & 1.62986500 & -5.30412000 & -0.54686800 \\ \mathrm{~N} & 1.59681000 & -2.05157700 & 0.31628500 \\ \mathrm{O} & -1.09917900 & -0.84661800 & 1.00898100 \\ \mathrm{C} & 1.81508600 & 0.32159100 & 1.61356400 \\ \mathrm{H} & 5.06493300 & 0.44832100 & 2.22042300 \\ \mathrm{C} & 5.34638200 & -2.56848600 & 0.72365900 \\ \mathrm{C} & 6.61195100 & -1.83738400 & 0.25631500 \\ \mathrm{H} & 5.54845400 & -3.56186700 & 1.14263500 \\ \mathrm{H} & 4.60141200 & -2.66420200 & -0.07956300 \\ \mathrm{H} & 7.53071400 & -2.26594900 & 0.68165100 \\ \mathrm{H} & 6.71606900 & -1.81976100 & -0.83691200 \\ \mathrm{C} & 4.88853100 & 2.05156700 & -0.96221000 \\ \mathrm{C} & 5.02037200 & 0.70993600 & -1.66918300 \\ \mathrm{H} & 5.07851500 & 2.91168500 & -1.61571900 \\ \mathrm{H} & 5.54594800 & 2.11425300 & -0.08292300 \\ \mathrm{H} & 4.80400300 & 0.78363600 & -2.74516400 \\ \mathrm{H} & 6.00088000 & 0.24743000 & -1.51684200 \\ \mathrm{~B} & 5.40220700 & -0.51132000 & 1.60798900 \\ \mathrm{~B} & 3.05829600 & 0.78362100 & -0.57973100 \\ \mathrm{O} & 4.80613800 & -1.72814000 & 1.73604900 \\ \mathrm{O} & 6.46240200 & -0.50547800 & 0.74236200 \\ \mathrm{O} & 3.52681000 & 2.07812500 & -0.52528600 \\ \mathrm{O} & 4.00338800 & -0.09747000 & -1.06709800\end{array}$

A1

Sum of electronic and zero-point Energies= $-1183.585962$

Sum of electronic and thermal Free Energies= $-1183.642971$

$\begin{array}{crrr}\text { Rh } & -0.43116900 & 0.14763800 & 0.02351700 \\ \mathrm{C} & 2.46769900 & 0.11067800 & -0.42394700 \\ \mathrm{C} & 1.50167400 & -1.89953100 & -1.25479100 \\ \mathrm{C} & -1.56874700 & 0.05487300 & -1.85912200 \\ \mathrm{C} & -2.05858800 & -0.94909300 & -0.95705900 \\ \mathrm{C} & -2.64992000 & -0.26677000 & 0.17951100 \\ \mathrm{C} & -2.49647100 & 1.12546400 & 0.00820900 \\ \mathrm{C} & -1.77122100 & 1.32723700 & -1.23452900 \\ \mathrm{C} & 3.73940700 & -0.28886800 & -0.82174400 \\ \mathrm{C} & 3.88280200 & -1.51886900 & -1.45240800 \\ \mathrm{C} & 2.76647600 & -2.32451300 & -1.66094200 \\ \mathrm{H} & 0.64594800 & -2.56193800 & -1.38886400 \\ \mathrm{H} & 4.61003700 & 0.34190800 & -0.64245800 \\ \mathrm{H} & 2.88203400 & -3.29839800 & -2.13531700 \\ \mathrm{H} & 4.86947600 & -1.84993600 & -1.77095300 \\ \mathrm{C} & 1.32060100 & -0.65946300 & -0.64585600 \\ \mathrm{C} & -1.39988300 & 2.65281700 & -1.80360500 \\ \mathrm{H} & -0.54758100 & 2.57079300 & -2.48840700 \\ \mathrm{H} & -2.24031000 & 3.09177700 & -2.36097700 \\ \mathrm{H} & -1.11841600 & 3.36455300 & -1.01730400 \\ \mathrm{C} & -0.96849500 & -0.18976100 & -3.20028000 \\ \mathrm{H} & -0.35630100 & -1.10003600 & -3.20649200 \\ \mathrm{H} & -1.74953300 & -0.30389900 & -3.96592400 \\ \mathrm{H} & -0.31698800 & 0.63729300 & -3.50670900 \\ \mathrm{C} & -2.15826300 & -2.41189800 & -1.21642800 \\ \mathrm{H} & -3.20681400 & -2.70766800 & -1.37246800 \\ \mathrm{H} & -1.60206600 & -2.69601800 & -2.11850300 \\ \mathrm{H} & -1.75386600 & -2.97396900 & -0.36571000 \\ \mathrm{C} & -2.97178200 & 2.19857600 & 0.92734600 \\ \mathrm{H} & -4.00549100 & 2.50035200 & 0.70115000 \\ \mathrm{H} & -2.94523600 & 1.87188800 & 1.97430100 \\ \mathrm{H} & -2.35253500 & 3.10182300 & 0.84882200 \\ \mathrm{O} & 0.18798200 & -0.63800200 & 1.88659400\end{array}$




$\begin{array}{lccc}\mathrm{C} & 0.04740700 & -1.89764400 & 2.13616300 \\ \mathrm{O} & -0.50399300 & -2.73505800 & 1.42277400 \\ \mathrm{C} & 0.66839500 & -2.30123000 & 3.46203700 \\ \mathrm{H} & 0.32477400 & -3.29767800 & 3.75530300 \\ \mathrm{H} & 1.76033400 & -2.31934200 & 3.35520100 \\ \mathrm{H} & 0.43155100 & -1.57127600 & 4.24399700 \\ \mathrm{C} & -3.28061800 & -0.96637000 & 1.33015100 \\ \mathrm{H} & -3.32152900 & -0.32762000 & 2.22036200 \\ \mathrm{H} & -4.31018400 & -1.26475800 & 1.08134700 \\ \mathrm{H} & -2.71134900 & -1.86952900 & 1.58043600 \\ \mathrm{~N} & 2.25792300 & 1.33788300 & 0.25574900 \\ \mathrm{C} & 3.10624300 & 2.27615500 & 0.73511200 \\ \mathrm{C} & 2.34605100 & 3.23260900 & 1.37735800 \\ \mathrm{H} & 4.17593400 & 2.19199000 & 0.59427700 \\ \mathrm{C} & 1.02247500 & 2.78785200 & 1.24609300 \\ \mathrm{H} & 2.70127400 & 4.12471100 & 1.87513900 \\ \mathrm{H} & 0.10171100 & 3.22334500 & 1.61752000 \\ \mathrm{~N} & 0.98412500 & 1.64910200 & 0.56302400\end{array}$

\section{A3}

Sum of electronic and zero-point Energies= $-1205.631173$

Sum of electronic and thermal Free Energies= $-1205.687440$

$\begin{array}{crrr}\text { Rh } & 0.43942400 & 0.15692900 & 0.02180400 \\ \mathrm{C} & -2.31529700 & -0.70808600 & 0.52264500 \\ \mathrm{C} & -0.77113200 & -2.52559300 & 0.86121800 \\ \mathrm{C} & 1.66452600 & -0.00996400 & 1.84810200 \\ \mathrm{C} & 2.35303000 & -0.65303600 & 0.76736000 \\ \mathrm{C} & 2.69563000 & 0.36883000 & -0.20271000 \\ \mathrm{C} & 2.21293400 & 1.61467400 & 0.25068900 \\ \mathrm{C} & 1.51072600 & 1.37146200 & 1.49698600 \\ \mathrm{C} & -3.37995000 & -1.54301100 & 0.88616700 \\ \mathrm{C} & -3.13912200 & -2.85962000 & 1.24500200 \\ \mathrm{C} & -1.83357300 & -3.35091600 & 1.22068200 \\ \mathrm{H} & 0.23567400 & -2.94022400 & 0.81151500 \\ \mathrm{H} & -4.40375600 & -1.16674500 & 0.88520700 \\ \mathrm{H} & -1.64318100 & -4.39313000 & 1.47688600 \\ \mathrm{H} & -3.96572200 & -3.50827200 & 1.52959200 \\ \mathrm{C} & -2.49169100 & 0.66331400 & 0.06367200 \\ \mathrm{C} & -3.71769800 & 1.30878000 & -0.13072700 \\ \mathrm{C} & -3.74435900 & 2.60070500 & -0.62563700 \\ \mathrm{H} & -4.64424300 & 0.78633300 & 0.09511800 \\ \mathrm{C} & -1.36622400 & 2.54548300 & -0.72911000 \\ \mathrm{C} & -2.54646300 & 3.23826800 & -0.94171900 \\ \mathrm{H} & -4.69586100 & 3.10694900 & -0.77846400 \\ \mathrm{H} & -0.39758100 & 2.98052700 & -0.97549800 \\ \mathrm{H} & -2.52423600 & 4.24479200 & -1.35087300 \\ \mathrm{~N} & -1.33809900 & 1.31115200 & -0.22077300 \\ \mathrm{C} & -0.99029900 & -1.18799500 & 0.53378000 \\ \mathrm{C} & 0.84932200 & 2.41349200 & 2.33216000 \\ \mathrm{H} & 0.05798500 & 1.98209900 & 2.95702200 \\ \mathrm{H} & 1.57370200 & 2.90471800 & 2.99837600 \\ \mathrm{H} & 0.38818800 & 3.19634000 & 1.71647500 \\ \mathrm{C} & 1.21276600 & -0.65603700 & 3.11237600 \\ \mathrm{H} & 0.88116500 & -1.68746700 & 2.94092600 \\ \mathrm{H} & 2.02482200 & -0.68192300 & 3.85352700 \\ \mathrm{H} & 0.36783800 & -0.11863800 & 3.55984500 \\ \mathrm{C} & 2.83427300 & -2.06182000 & 0.71867700 \\ \mathrm{H} & 3.93155100 & -2.10043700 & 0.79529900 \\ \mathrm{H} & 2.42994200 & -2.65194400 & 1.55054500 \\ \mathrm{H} & 2.53020100 & -2.53181700 & -0.22520500 \\ \mathrm{C} & 2.38803400 & 2.93411900 & -0.42142700 \\ \mathrm{H} & 3.36602200 & 3.38237400 & -0.19063900 \\ \mathrm{H} & 2.32033500 & 2.84372500 & -1.51382000 \\ & 1.62749700 & 3.65840500 & -0.10019000\end{array}$

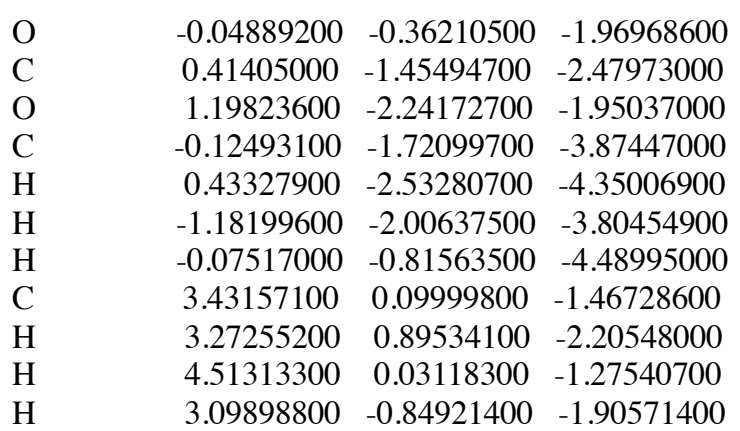

A2

Sum of electronic and zero-point Energies $=$ $-1205.854108$

Sum of electronic and thermal Free Energies= $-1205.914832$

\begin{tabular}{|c|c|c|c|}
\hline $\mathrm{Rh}$ & -0.60329400 & 0.17389700 & -0.07130900 \\
\hline $\mathrm{C}$ & 2.15942000 & -0.46087100 & -0.69872700 \\
\hline $\mathrm{C}$ & 0.83419900 & -2.46931500 & -0.73495500 \\
\hline $\mathrm{C}$ & -2.13829600 & -0.48348600 & -1.48993400 \\
\hline $\mathrm{C}$ & -2.49185600 & -0.87706000 & -0.15147300 \\
\hline $\mathrm{C}$ & -2.74764200 & 0.33678800 & 0.61611400 \\
\hline $\mathrm{C}$ & -2.49007800 & 1.44900100 & -0.20406800 \\
\hline $\mathrm{C}$ & -2.04941500 & 0.94258200 & -1.49599800 \\
\hline $\mathrm{C}$ & 3.27312400 & -1.18325400 & -1.14933500 \\
\hline $\mathrm{C}$ & 3.16578900 & -2.54637100 & -1.38121500 \\
\hline $\mathrm{C}$ & 1.95131900 & -3.19115500 & -1.14474200 \\
\hline $\mathrm{H}$ & -0.10272700 & -2.99474900 & -0.54412400 \\
\hline $\mathrm{H}$ & 4.21802500 & -0.67855900 & -1.34981800 \\
\hline $\mathrm{H}$ & 1.87401300 & -4.26845200 & -1.29217000 \\
\hline $\mathrm{H}$ & 4.02604900 & -3.10825200 & -1.74070300 \\
\hline $\mathrm{C}$ & 0.90728300 & -1.08859200 & -0.55054400 \\
\hline $\mathrm{C}$ & -1.65479100 & 1.81135400 & -2.63808600 \\
\hline $\mathrm{H}$ & -1.11960500 & 1.24717000 & -3.41025200 \\
\hline $\mathrm{H}$ & -2.53902800 & 2.27135100 & -3.10391200 \\
\hline $\mathrm{H}$ & -0.99394300 & 2.62033500 & -2.30267700 \\
\hline $\mathrm{C}$ & -1.90931900 & -1.39448800 & -2.64521000 \\
\hline $\mathrm{H}$ & -1.41840600 & -2.32521700 & -2.33534600 \\
\hline $\mathrm{H}$ & -2.85921000 & -1.66301300 & -3.12969000 \\
\hline $\mathrm{H}$ & -1.26590300 & -0.92952000 & -3.40165400 \\
\hline $\mathrm{C}$ & -2.77022500 & -2.25683500 & 0.33200500 \\
\hline $\mathrm{H}$ & -3.84552500 & -2.39354800 & 0.52266100 \\
\hline $\mathrm{H}$ & -2.47089700 & -3.00768300 & -0.41006600 \\
\hline $\mathrm{H}$ & -2.21794900 & -2.44594400 & 1.26134300 \\
\hline $\mathrm{C}$ & -2.55377700 & 2.89392500 & 0.15356900 \\
\hline $\mathrm{H}$ & -3.36853800 & 3.40626900 & -0.37974000 \\
\hline $\mathrm{H}$ & -2.71916700 & 3.03456900 & 1.22762500 \\
\hline $\mathrm{H}$ & -1.61730600 & 3.40825400 & -0.10324000 \\
\hline $\mathrm{O}$ & 0.37564800 & 0.21783600 & 1.79581400 \\
\hline $\mathrm{C}$ & 0.20553100 & -0.73924100 & 2.64715400 \\
\hline $\mathrm{O}$ & -0.56910000 & -1.68925100 & 2.54449800 \\
\hline $\mathrm{C}$ & 1.09919200 & -0.59684000 & 3.86749500 \\
\hline $\mathrm{H}$ & 0.84550000 & -1.35101600 & 4.61808900 \\
\hline $\mathrm{H}$ & 2.14760100 & -0.72259000 & 3.56826600 \\
\hline $\mathrm{H}$ & 1.00567900 & 0.40734900 & 4.29725500 \\
\hline $\mathrm{C}$ & -3.15283200 & 0.33101800 & 2.04697600 \\
\hline $\mathrm{H}$ & -2.97406200 & 1.30100300 & 2.52502600 \\
\hline $\mathrm{H}$ & -4.22352900 & 0.09655300 & 2.14385300 \\
\hline $\mathrm{H}$ & -2.58547500 & -0.43173000 & 2.59479400 \\
\hline $\mathrm{C}$ & 2.13824400 & 0.99156500 & -0.44329200 \\
\hline $\mathrm{O}$ & 1.04525300 & 1.59928600 & -0.52854200 \\
\hline $\mathrm{N}$ & 3.26275200 & 1.69246100 & -0.14833100 \\
\hline $\mathrm{C}$ & 4.42815000 & 1.12275400 & 0.50455900 \\
\hline $\mathrm{H}$ & 5.32354700 & 1.18472900 & -0.13074900 \\
\hline $\mathrm{H}$ & 4.62409300 & 1.68302400 & 1.42965500 \\
\hline
\end{tabular}




$\begin{array}{lrrr}\mathrm{H} & 4.25056000 & 0.07990600 & 0.77546200 \\ \mathrm{C} & 3.14999700 & 3.13537600 & -0.03342400 \\ \mathrm{H} & 4.13272700 & 3.58107600 & -0.22906300 \\ \mathrm{H} & 2.42860200 & 3.51181700 & -0.76121900 \\ \mathrm{H} & 2.81531000 & 3.43018800 & 0.97224200\end{array}$

\section{A4}

Sum of electronic and zero-point Energies=

$-1166.614133$

Sum of electronic and thermal Free Energies= $-1166.670569$

\begin{tabular}{|c|c|c|c|}
\hline $\mathrm{Rh}$ & -0.38653000 & -0.14915000 & -0.14121100 \\
\hline $\mathrm{C}$ & 2.62981800 & -0.10909100 & 0.11150200 \\
\hline $\mathrm{C}$ & 1.55461200 & 1.86618400 & 0.90573900 \\
\hline $\mathrm{C}$ & -0.89880000 & -0.66464800 & 1.92538900 \\
\hline $\mathrm{C}$ & -1.71392000 & 0.43141600 & 1.47155700 \\
\hline $\mathrm{C}$ & -2.60108700 & -0.07567600 & 0.42537600 \\
\hline $\mathrm{C}$ & -2.27930100 & -1.41709700 & 0.18271900 \\
\hline $\mathrm{C}$ & -1.17352400 & -1.77096700 & 1.07001300 \\
\hline $\mathrm{C}$ & 3.86221200 & 0.38721000 & 0.55175200 \\
\hline $\mathrm{C}$ & 3.93771800 & 1.62238000 & 1.17612900 \\
\hline $\mathrm{C}$ & 2.77671700 & 2.37253100 & 1.33941700 \\
\hline $\mathrm{H}$ & 0.65457600 & 2.47446100 & 1.00251600 \\
\hline $\mathrm{H}$ & 4.77022100 & -0.19900300 & 0.38995900 \\
\hline $\mathrm{H}$ & 2.82168600 & 3.35840700 & 1.80072000 \\
\hline $\mathrm{H}$ & 4.90003200 & 2.00152500 & 1.51543300 \\
\hline $\mathrm{C}$ & 1.44414000 & 0.60430700 & 0.31133100 \\
\hline $\mathrm{C}$ & -0.54186200 & -3.11850700 & 1.12533800 \\
\hline $\mathrm{H}$ & 0.48469400 & -3.06623700 & 1.51068900 \\
\hline $\mathrm{H}$ & -1.11069600 & -3.79733300 & 1.77807700 \\
\hline $\mathrm{H}$ & -0.50651400 & -3.57527900 & 0.12792700 \\
\hline $\mathrm{C}$ & 0.06681000 & -0.63494000 & 3.05927400 \\
\hline $\mathrm{H}$ & 0.55170200 & 0.34482200 & 3.14825500 \\
\hline $\mathrm{H}$ & -0.43839200 & -0.85134400 & 4.01162200 \\
\hline $\mathrm{H}$ & 0.86441200 & -1.37679600 & 2.92559900 \\
\hline $\mathrm{C}$ & -1.85500000 & 1.76246800 & 2.12137200 \\
\hline $\mathrm{H}$ & -2.78964800 & 1.80957600 & 2.70131000 \\
\hline $\mathrm{H}$ & -1.02696300 & 1.96146400 & 2.81250800 \\
\hline $\mathrm{H}$ & -1.86667900 & 2.55237900 & 1.36105500 \\
\hline $\mathrm{C}$ & -2.84519500 & -2.33187400 & -0.84773600 \\
\hline $\mathrm{H}$ & -3.32509100 & -3.20854100 & -0.38813100 \\
\hline $\mathrm{H}$ & -3.59577000 & -1.82602400 & -1.46496600 \\
\hline $\mathrm{H}$ & -2.05944300 & -2.69967200 & -1.52319300 \\
\hline $\mathrm{O}$ & -0.32728300 & 1.16124900 & -1.77905800 \\
\hline $\mathrm{C}$ & -0.69047900 & 2.39371700 & -1.68561200 \\
\hline $\mathrm{O}$ & -1.15872600 & 2.95481800 & -0.69158600 \\
\hline $\mathrm{C}$ & -0.46703200 & 3.17139000 & -2.97028200 \\
\hline $\mathrm{H}$ & -1.02710000 & 4.11115800 & -2.94985000 \\
\hline $\mathrm{H}$ & 0.60204500 & 3.39981400 & -3.06711400 \\
\hline $\mathrm{H}$ & -0.75217300 & 2.57531800 & -3.84404900 \\
\hline $\mathrm{C}$ & -3.61578700 & 0.76561000 & -0.26274800 \\
\hline $\mathrm{H}$ & -3.98465600 & 0.29525400 & -1.18118500 \\
\hline $\mathrm{H}$ & -4.47800400 & 0.94591400 & 0.39684200 \\
\hline $\mathrm{H}$ & -3.18039700 & 1.73870900 & -0.52174900 \\
\hline $\mathrm{C}$ & 1.77397200 & -1.93823000 & -1.36755400 \\
\hline $\mathrm{N}$ & 2.68350600 & -1.36945500 & -0.55185000 \\
\hline $\mathrm{H}$ & 3.59344400 & -1.81296300 & -0.53347100 \\
\hline $\mathrm{O}$ & 0.62381000 & -1.51939600 & -1.54907400 \\
\hline $\mathrm{C}$ & 2.21764800 & -3.17583000 & -2.09775500 \\
\hline $\mathrm{H}$ & 2.19596300 & -2.97342900 & -3.17481900 \\
\hline $\mathrm{H}$ & 3.22093300 & -3.51921500 & -1.82277900 \\
\hline $\mathrm{H}$ & 1.49873800 & -3.98053900 & -1.90736200 \\
\hline
\end{tabular}




\section{References}

1. Lin, W.; Li, W.; Lu, D.; Su, F.; Wen, T.-Bin; Zhang, H.-Jun Dual Effects of Cyclopentadienyl Ligands on Rh(III)-Catalyzed Dehydrogenative Arylation of Electron-Rich Alkenes. ACS Catal. 2018, 8, 8070-8076.

2. Shibata, Y.; Tanaka, K. Catalytic $[2+2+1]$ Cross-Cyclotrimerization of Silylacetylenes and Two Alkynyl Esters To Produce Substituted Silylfulvenes. Angew. Chem., Int. Ed. 2011, 50, 10917-10921.

3. Tanaka, J.; Shibata, Y.; Joseph, A.; Nogami, J.; Terasawa, J.; Yoshimura, R.; Tanaka, K. Rhodium(III)-Catalyzed Ortho-Bromination of Phenyl Carbamates Accelerated by a Secondary Amide-Pendant Cyclopentadienyl Ligand. Chem. - Eur. J. 2020, 26, 5774-5779.

4. Yoshizaki, S.; Shibata, Y.; Tanaka, K. Fulvene Synthesis by Rhodium(I)-Catalyzed [2+2+1] Cycloaddition: Synthesis and Catalytic Activity of Tunable Cyclopentadienyl Rhodium(III) Complexes with Pendant Amides. Angew. Chem., Int. Ed. 2017, 56, 3590-3593.

5. Sasmal, S.; Sinha, S. K.; Lahiri, G. K.; Maiti, D. A directing group-assisted rutheniumcatalyzed approach to access meta-nitrated phenols. Chem. Commun. 2020, 56, 7100-7103.

6. Wang, P.; Farmer, M. E.; Huo, X.; Jain, P.; Shen, P.-X.; Ishoey, M.; Bradner, J. E.; Wisniewski, S. R.; Eastgate, M. D.; Yu, J.-Q. Ligand-Promoted Meta-C-H Arylation of Anilines, Phenols, and Heterocycles. J. Am. Chem. Soc. 2016, 138, 9269-9276

7. Nwachukwu, C. I.; McFadden, T. P.; Roberts, A. G. Ni-Catalyzed Iterative Alkyl Transfer from Nitrogen Enabled by the In Situ Methylation of Tertiary Amines. J. Org. Chem. 2020, 85, 9979-9992.

8. Sikari, R.; Sinha, S.; Chakraborty, G.; Das, S.; van Leest, N. P.; Paul, N. D. C-N CrossCoupling Reactions Under Mild Conditions Using Singlet Di-Radical Nickel(II)-Complexes as Catalyst: N-Arylation and Quinazoline Synthesis. Adv. Synth. Catal. 2019, 361, 4342-4353.

9. Li, Z.-Y.; Lakmal, H. H. C.; Cui, X. Enabling Catalytic Arene C-H Amidomethylation via Bis(tosylamido)methane as a Sustainable Formaldimine Releaser. Org. Lett. 2019, 21, 37353740.

10. Yuan, C.; Zhao, Y.; Zheng, L. $\alpha$-D-Galacturonic Acid as Natural Ligand for Selective CopperCatalyzed $N$-Arylation of $N$-Containing Heterocycles. Synlett 2019, 30, 2173-2180.

11. Fernández-Salas, J. A.; Manzini, S.; Piola, L.; Slawin, A. M. Z.; Nolan, S. P. Ruthenium catalysed C-H bond borylation. Chem. Commun. 2014, 50, 6782-6784.

12. Zhou, Q.; Du, F.; Chen, Y.; Fu, Y.; Sun, W.; Wu, Y.; Chen, G. L-(-)-Quebrachitol as a Ligand for Selective Copper(0)-Catalyzed N-Arylation of Nitrogen-Containing Heterocycles. J. Org. Chem. 2019, 84, 8160-8167.

13. Vantourout, J. C.; Miras, H. N.; Isidro-Llobet, A.; Sproules, S.; Watson, A. J. B. Spectroscopic Studies of the Chan-Lam Amination: A Mechanism-Inspired Solution to Boronic Ester Reactivity. J. Am. Chem. Soc. 2017, 139, 4769-4779.

14. Zhao, L.-L.; Liu, W.; Zhang, Z.; Zhao, H.; Wang, Q.; Yan, X. Ruthenium-Catalyzed orthoand meta-H/D Exchange of Arenes. Org. Lett. 2019, 21, 10023-10027.

15. Vorobyeva, E.; Gerken, V. C.; Mitchell, S.; Sabadell-Rendón, A.; Hauert, R.; Xi, S.; Borgna, A.; Klose, D.; Collins, S. M.; Midgley, P. A.; Kapaptsoglou, D. M.; Ramasse, Q. M.; RuizFerrando, A.; Fako, E.; Ortuño, M. A.; López, N.; Carreira, E. M.; Pérez-Ramírez, J. Activation of Copper Species on Carbon Nitride for Enhanced Activity in the Arylation of Amines. ACS Catal. 2020, 10, 11069-11080.

16. Kawamorita, S.; Miyazaki, T.; Ohmiya, H.; Iwai, T.; Sawamura, M. Rh-Catalyzed OrthoSelective $\mathrm{C}-\mathrm{H}$ Borylation of $N$-Functionalized Arenes with Silica-Supported Bridgehead Monophosphine Ligands. J. Am. Chem. Soc. 2011, 133, 19310-19313.

17. Fukata, G.; Itoh, T.; Tashiro, M. Cyclodienones. 9. Reaction of 4-Halo-2,4,6-tri-tert-butyl-2,5cyclohexadien-1-ones with Pyrazoles and Preparation of 1-(2-Hydroxyphenyl)- and 1-(4Hydroxyphenyl)pyrazoles. Heterocycles 1982, 19, 1487-1495.

18. Wu, Y.; Zhou, B. Rhodium(III)-Catalyzed Selective C-H Acetoxylation and Hydroxylation 
Reactions. Org. Lett. 2017, 19, 3532-3535.

19. Okada, S.; Namikoshi, T.; Watanabe, S.; Murata, M. Ruthenium-Catalyzed Ortho-Selective Aromatic C-H Borylation of 2-Arylpyridines with Pinacolborane. ChemCatChem 2015, 7, 1531-1534.

20. Yamaguchi, T.; Yamaguchi, E.; Toda, N.; Itoh, A. Direct ortho-Hydroxylation of 2Phenylpyridines using Palladium(II) Chloride and Hydrogen Peroxide. Adv. Synth. Catal. 2015, 357, 2017-2021.

21. Chen, C.; Pan, Y.; Zhao, H.; Xu, X.; Luo, Z.; Cao, L.; Xi, S.; Li, H.; Xu, L. Ruthenium(II)Catalyzed Regioselective C-8 Hydroxylation of 1,2,3,4-Tetrahydroquinolines. Org. Lett. 2018, 20, 6799-6803.

22. Thongpaen, J.; Schmid, T. E.; Toupet, L.; Dorcet, V.; Mauduit, M.; Baslé, O. Directed ortho $\mathrm{C}-\mathrm{H}$ borylation catalyzed using $\mathrm{Cp} * \mathrm{Rh}(\mathrm{III})-\mathrm{NHC}$ complexes. Chem. Commun. 2018, 54, 8202-8205.

23. Cody, J.; Dennisson, J.; Gilmore, J.; VanDerveer, D. G.; Henary, M. M.; Gabrielli, A.; Sherrill, C. D.; Zhang, Y.; Pan, C.-P.; Burda, C.; Fahrni, C. J. X-ray Structures, Photophysical Characterization, and Computational Analysis of Geometrically Constrained Copper(I)Phenanthroline Complexes. Inorg. Chem. 2003, 42, 4918-4929.

24. González-Pelayo, S.; López, L. A. Microwave-Assisted Generation and Capture by Azoles of ortho-Quinone Methide Intermediates under Aqueous Conditions. Eur. J. Org. Chem. 2017, 2017, 6003-6007.

25. Ros, A.; Estepa, B.; López-Rodríguez, R.; Álvarez, E.; Fernández, R.; Lassaletta, J. M. Use of Hemilabile N,N Ligands in Nitrogen-Directed Iridium-Catalyzed Borylations of Arenes. Angew. Chem., Int. Ed. 2011, 50, 11724-11728.

26. Dong, J.; Liu, P.; Sun, P. Palladium-Catalyzed Aryl C $\left(\mathrm{sp}^{2}\right)-\mathrm{H}$ Bond Hydroxylation of 2Arylpyridine Using TBHP as Oxidant. J. Org. Chem. 2015, 80, 2925-2929.

27. Roering, A. J.; Hale, L. V. A.; Squier, P. A.; Ringgold, M. A.; Wie-derspan, E. R.; Clark, T. B. Iridium-Catalyzed, Substrate-Directed C-H Borylation Reactions of Benzylic Amines. Org. Lett. 2012, 14, 3558-3561.

28. Hamid, M. H. S. A.; Allen, C. L.; Lamb, G. W.; Maxwell, A. C.; Maytum, H. C.; Watson, A. J. A.; Williams, J. M. J. Ruthenium-Catalyzed $N$-Alkylation of Amines and Sulfonamides Using Borrowing Hydrogen Methodology. J. Am. Chem. Soc. 2009, 131, 1766-1774.

29. Kim, S.; Hong, S. H. Ruthenium-Catalyzed Aminomethylation and Methylation of Phenol Derivatives Utilizing Methanol as the $\mathrm{C}_{1}$ Source. Adv. Synth. Catal. 2017, 359, 798-810.

30. Sarkar, S. D.; Kumar, N. Y. P.; Ackermann, L. Ruthenium(II) Biscarboxylate-Catalyzed Borylations of $\mathrm{C}\left(\mathrm{sp}^{2}\right)-\mathrm{H}$ and $\mathrm{C}\left(\mathrm{sp}^{3}\right)-\mathrm{H}$ Bonds. Chem. - Eur. J. 2017, 23, 84-87.

31. Massignan, L.; Tan, X.; Meyer, T. H.; Kuniyil, R.; Messinis, A. M.; Ackermann, L. C-H Oxygenation Reactions Enabled by Dual Catalysis with Electrogenerated Hypervalent Iodine Species and Ruthenium Complexes. Angew. Chem., Int. Ed. 2020, 59, 3184-3189.

32. Henry, M. C.; McGrory, R.; Faggyas, R. J.; Mostafa, M. A. B.; Sutherland, A. One-pot orthoamination of aryl $\mathrm{C}-\mathrm{H}$ bonds using consecutive iron and copper catalysis. Org. Biomol. Chem. 2019, 17, 4629-4639.

33. Guo, W.-H.; Min, Q.-Q.; Gu, J.-W .; Zhang, X. Rhodium-Catalyzed ortho-Selective C-F Bond Borylation of Polyfluoroarenes with Bpin-Bpin. Angew. Chem., Int. Ed. 2015, 54, 9075-9078.

34. Sharma, R.; Patel, N.; Vishwakarma, R. A.; Bharatam, P. V.; Bharate, S. B. Metal-free oxidative cyclization of acetophenones with diamines: a facile access to phenylpyridines. Chem. Commun. 2016, 52, 1009-1012.

35. Zhong, L.; Zong, Z.-H.; Wang, X.-C. $N$-heterocyclic carbene enabled rhodium-catalyzed ortho $\mathrm{C}\left(s p^{2}\right)-\mathrm{H}$ borylation at room temperature. Tetrahedron 2019, 75, 2547-2552.

36. Novikov, R. A.; Klimenko, I. P.; Shulishov, E. V.; Korolev, V. A.; Tomilov, Y. V. Thermal transformation of cyclopropylazoarenes into the five-membered nitrogen-containing heterocycles. Russ. Chem. Bull., Int. Ed. 2008, 57, 1718-1724. 
37. Kour, J.; Venkateswarlu, V.; Verma, P. K.; Hussain, Y.; Dubey, G.; Bharatam, P. V.; Sahoo, S. C.; Sawant, S. D. Oxone-DMSO Triggered Methylene Insertion and $\mathrm{C}\left(\mathrm{sp}^{2}\right)-\mathrm{C}\left(\mathrm{sp}^{3}\right)-\mathrm{H}-$ $\mathrm{C}\left(\mathrm{sp}^{2}\right)$ Bond Formation to Access Functional Bis-Heterocycles. J. Org. Chem. 2020, 85, 49514962.

38. Kwak, S. H.; Gulia, N.; Daugulis, O. Synthesis of Unsymmetrical 2,6-Diarylanilines by Palladium-Catalyzed C-H Bond Functionalization Methodology. J. Org. Chem. 2018, 83, 5844-5850.

39. Shibata, K.; Natsui, S.; Chatani, N. Rhodium-Catalyzed Alkenylation of C-H Bonds in Aromatic Amides with Alkynes. Org. Lett. 2017, 19, 2234-2237.

40. Qiu, Y.; Scheremetjew, A.; Ackermann, L. Electro-Oxidative C-C Alkenylation by Rhodium(III) Catalysis. J. Am. Chem. Soc. 2019, 141, 2731-2738.

41. Wu, Z. J.; Su, F.; Lin, W.; Song, J.; Wen, T. Bin; Zhang, H. J.; Xu, H. C. Scalable Rhodium(III)-Catalyzed Aryl C-H Phosphorylation Enabled by Anodic Oxidation Induced Reductive Elimination. Angew. Chem. Int. Ed. 2019, 58, 16770.

42. Gaussian 09, Revision B.01, Frisch, M. J.; Trucks, G. W.; Schlegel, H. B.; Scuseria, G. E.; Robb, M. A.; Cheeseman, J. R.; Scalmani, G.; Barone, V.; Mennucci, B.; Petersson, G. A.; Nakatsuji, H.; Caricato, M.; Li, X.; Hratchian, H. P.; Izmaylov, A. F.; Bloino, J.; Zheng, G.; Sonnenberg, J. L.; Hada, M.; Ehara, M.; Toyota, K.; Fukuda, R.; Hasegawa, J.; Ishida, M.; Nakajima, T.; Honda, Y.; Kitao, O.; Nakai, H.; Vreven, T.; Montgomery, Jr., J. A.; Peralta, J. E.; Ogliaro, F.; Bearpark, M.; Heyd, J. J.; Brothers, E.; Kudin, K. N.; Staroverov, V. N.; Kobayashi, R.; Normand, J.; Raghavachari, K.; Rendell, A.; Burant, J. C.; Iyengar, S. S.; Tomasi, J.; Cossi, M.; Rega, N.; Millam, N. J.; Klene, M.; Knox, J. E.; Cross, J. B.; Bakken, V.; Adamo, C.; Jaramillo, J.; Gomperts, R.; Stratmann, R. E.; Yazyev, O.; Austin, A. J.; Cammi, R.; Pomelli, C.; Ochterski, J. W.; Martin, R. L.; Morokuma, K.; Zakrzewski, V. G.; Voth, G. A.; Salvador, P.; Dannenberg, J. J.; Dapprich, S.; Daniels, A. D.; Farkas, Ö.; Foresman, J. B.; Ortiz, J. V.; Cioslowski, J.; Fox, D. J. Gaussian, Inc., Wallingford CT, 2009.

43. (a) Beche, A. D. Phys. Rev. 1988, A38, 3098-3100. (b) Beche, A. D. J. Chem. Phys. 1993, 98. 1372-1377. (c) Beche, A. D. J. Chem. Phys. 1993, 98, 5648-5652. (d) Lee, C; Yang, W.; Parr, R. G. Phys. Rev. 1988, B37, 785-788.

44. Tomasi, J.; Persico, M. Chem. Rev. 1994, 94, 2027-2094.

45. (a) Fukui, K. Acc. Chem. Res. 1981, 14, 363-368. (b) Ishida, K.; Morokuma, K.; Komornicki, A. J. Chem. Phys. 1977, 66, 2153-2156. (c) Gonzalez, C.; Schlegel, H. B. J. Chem. Phys. 1989, 90, 2154-2161. (d) Schlegel, H. B.; Gonzalez, C. J. Phys. Chem. 1990, 94, 5523-5527. 


\section{NMR Spectra}

${ }^{1} \mathrm{H}$ NMR $\left(\mathrm{CDCl}_{3}, 400 \mathrm{MHz}\right)$ of 2-(1H-Pyrazol-1-yl)phenol (4a)
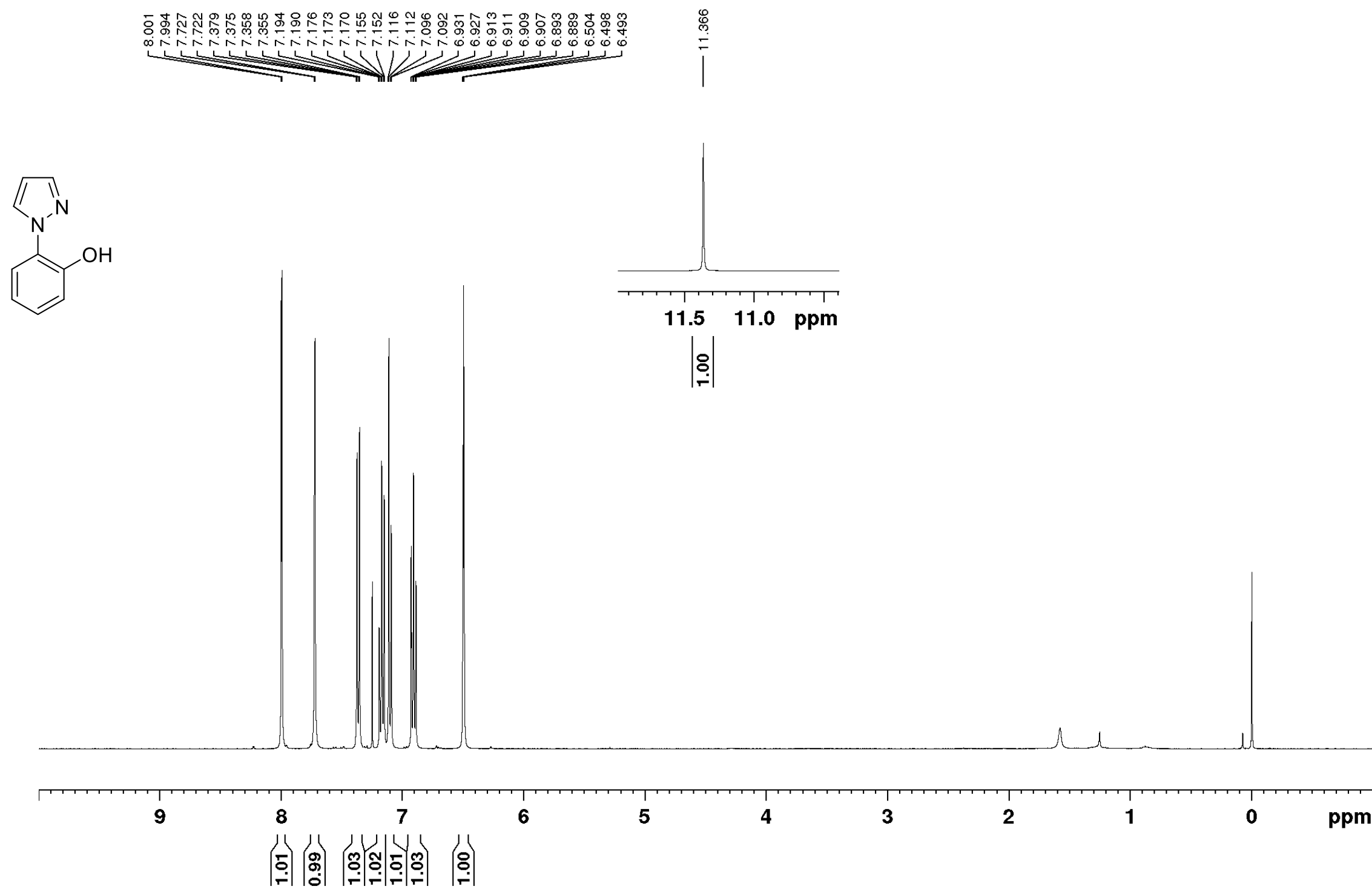
${ }^{13} \mathrm{C}$ NMR $\left(\mathrm{CDCl}_{3}, 100 \mathrm{MHz}\right)$ of 2-(1H-Pyrazol-1-yl)phenol (4a)
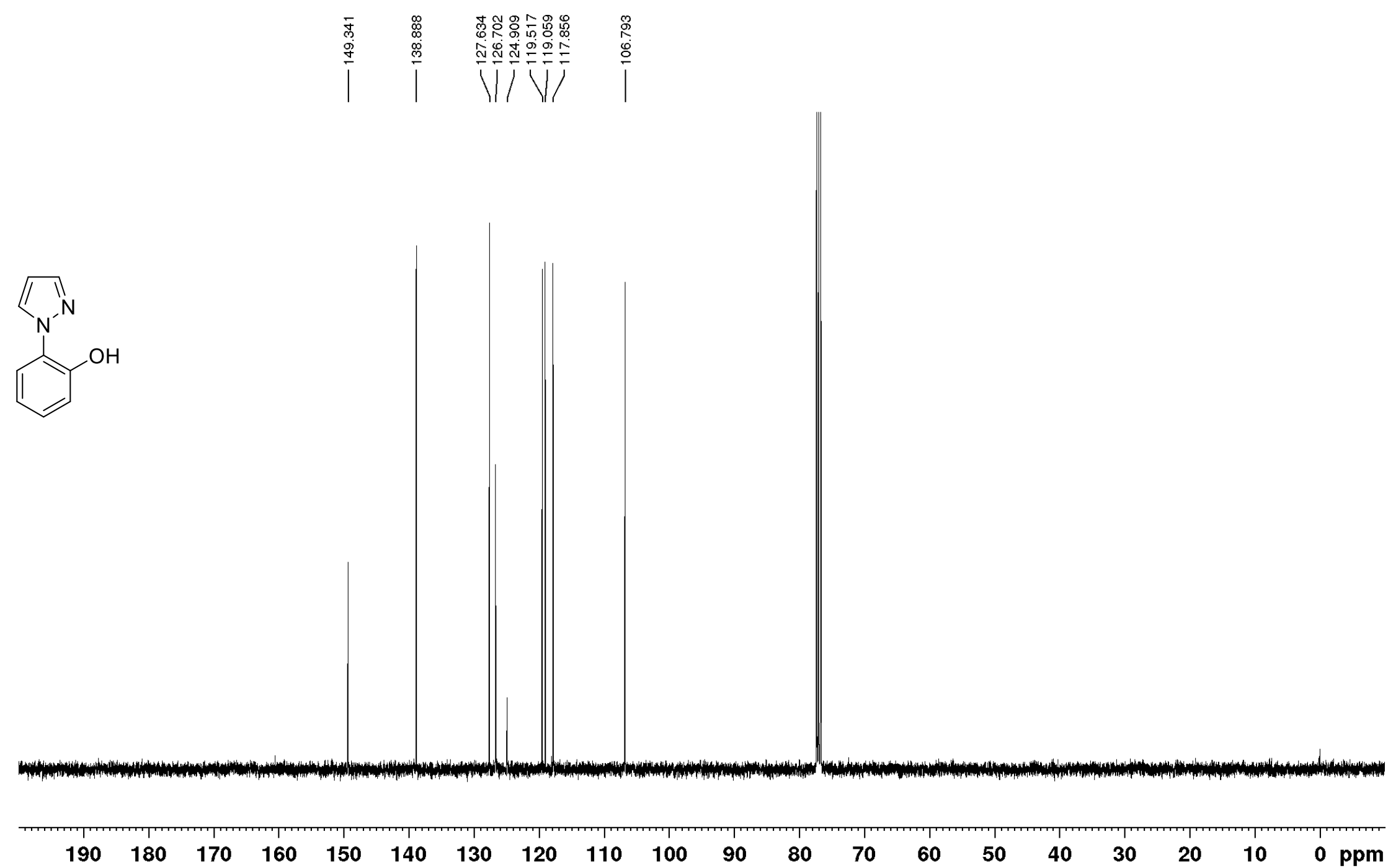

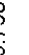


${ }^{1} \mathrm{H}$ NMR $\left(\mathrm{CDCl}_{3}, 400 \mathrm{MHz}\right)$ of 2-(3,5-Dimethyl-1H-pyrazol-1-yl)phenol (4b)

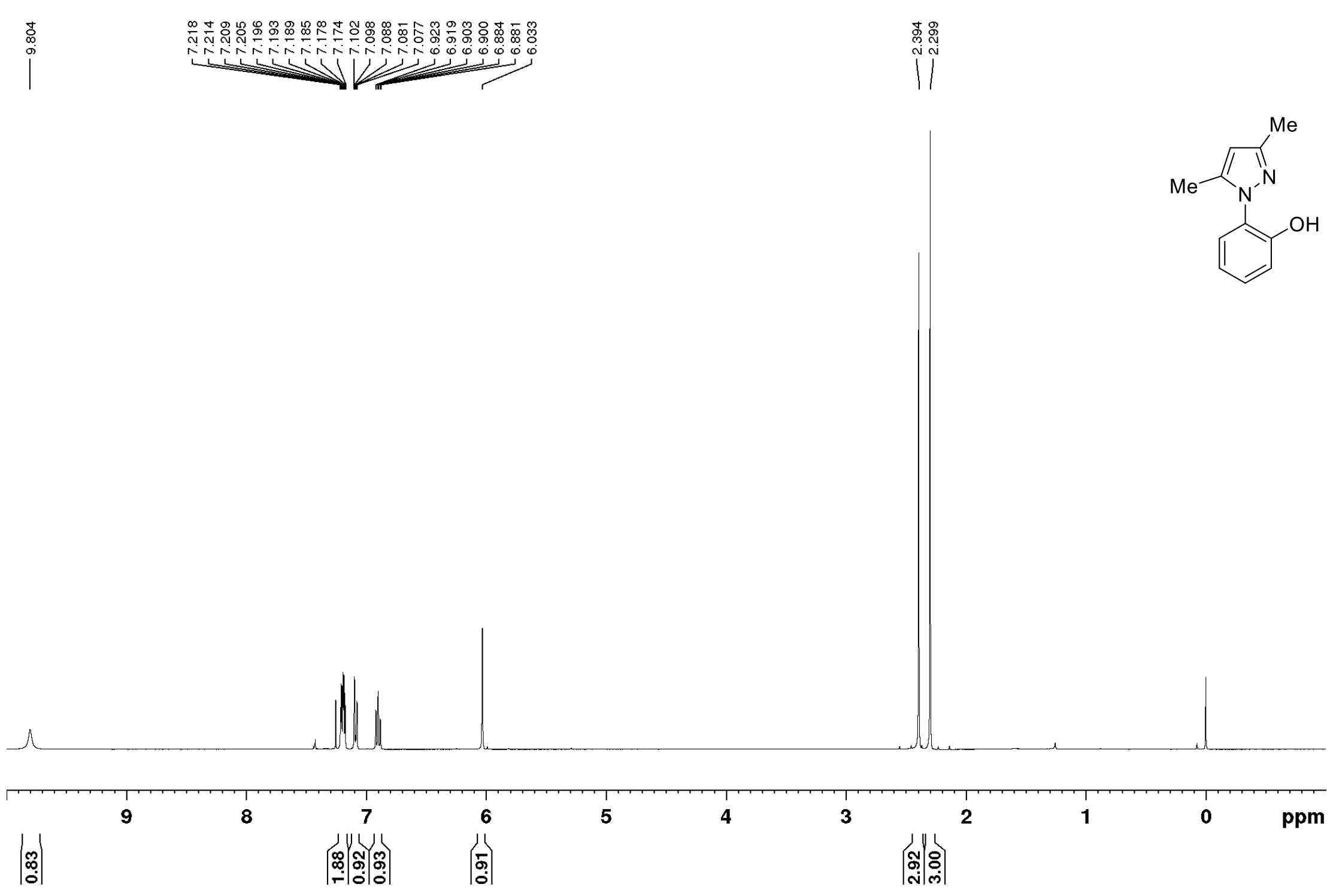


${ }^{13} \mathrm{C}$ NMR $\left(\mathrm{CDCl}_{3}, 100 \mathrm{MHz}\right)$ of 2-(3,5-Dimethyl-1 $\mathrm{H}$-pyrazol-1-yl)phenol (4b)

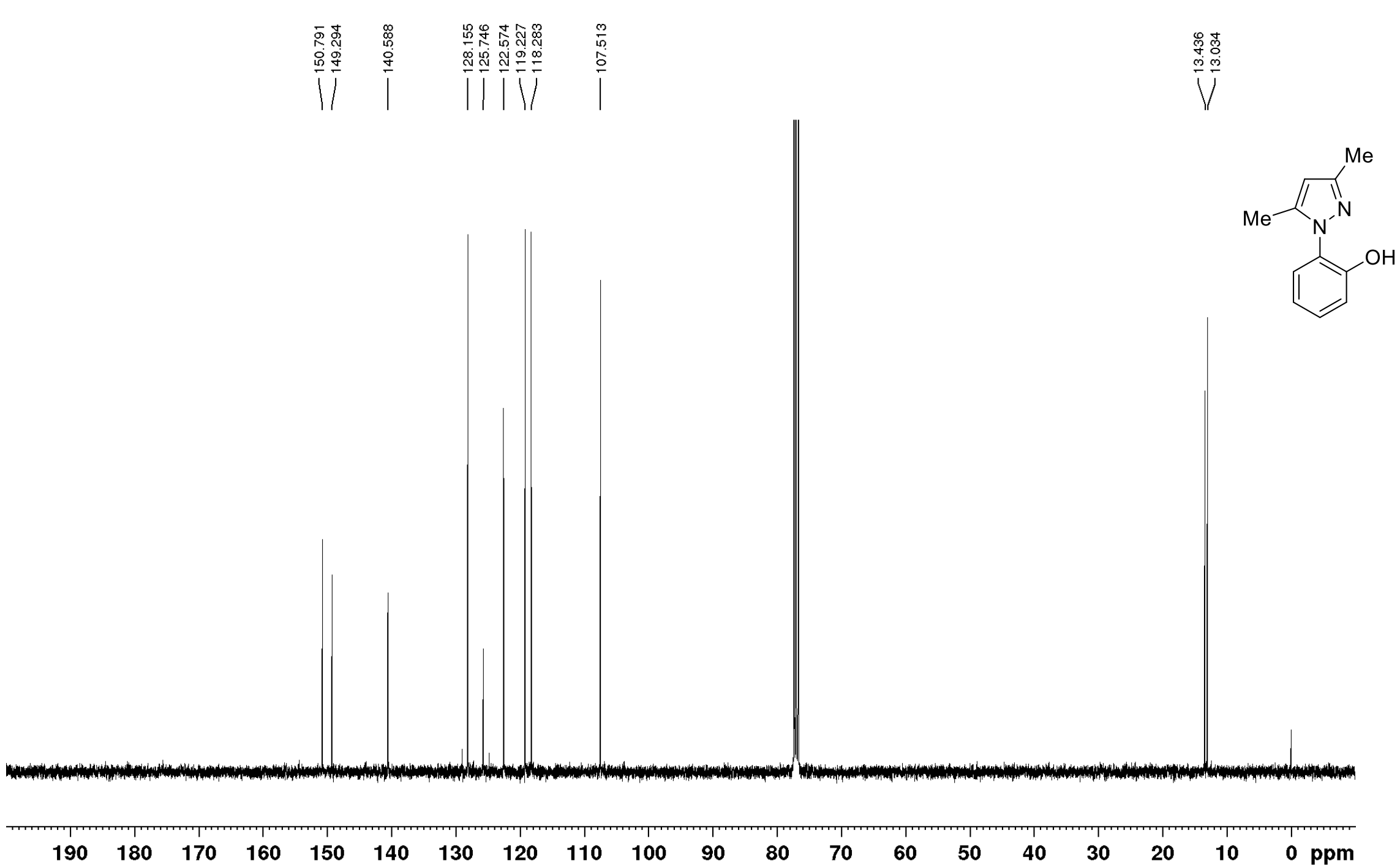


${ }^{1} \mathrm{H}$ NMR $\left(\mathrm{CDCl}_{3}, 400 \mathrm{MHz}\right)$ of 2-(Pyridin-2-yl)phenol (4c)
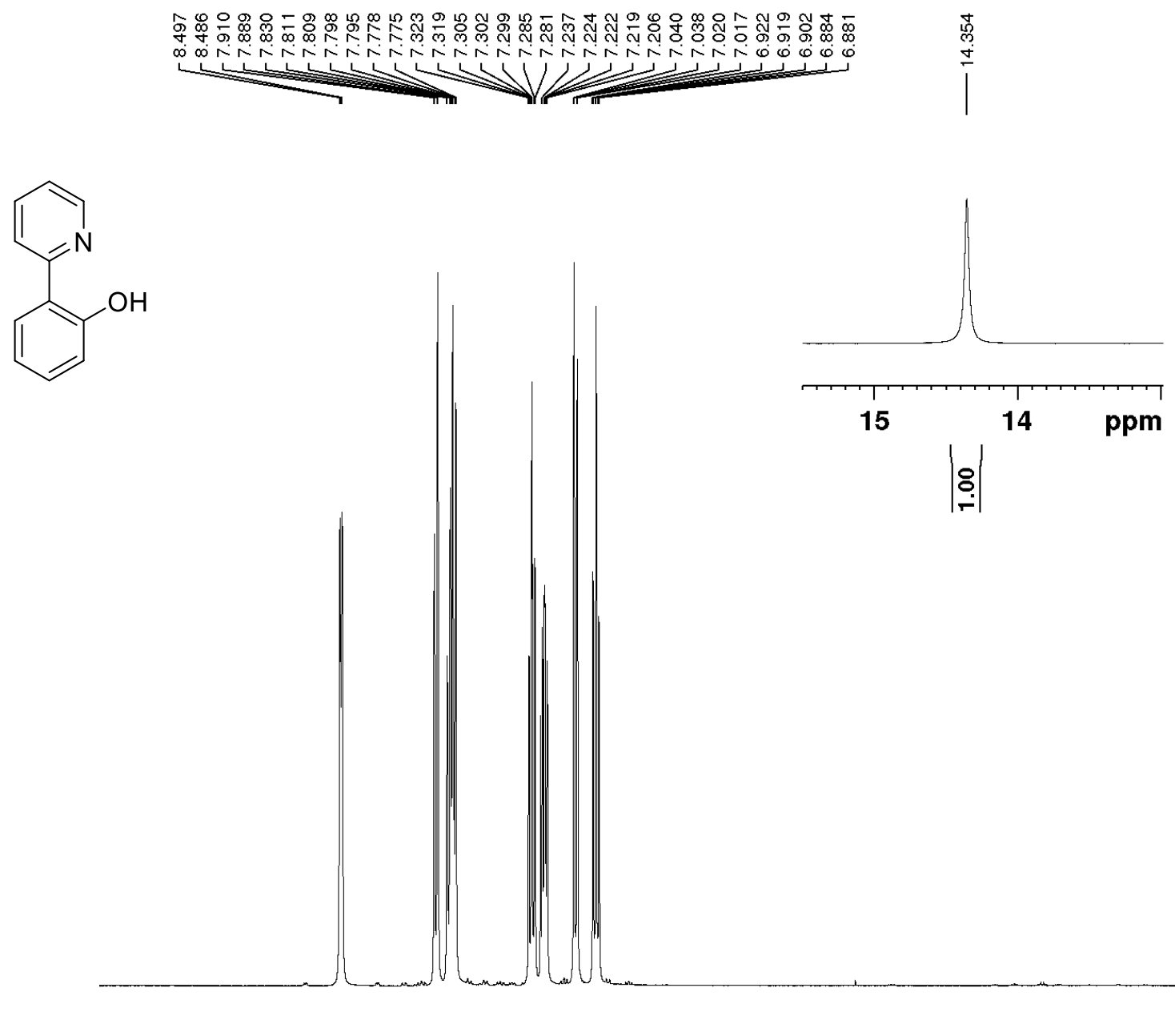

9
(ั) 
${ }^{13} \mathrm{C} \mathrm{NMR}\left(\mathrm{CDCl}_{3}, 100 \mathrm{MHz}\right)$ of 2-(Pyridin-2-yl)phenol (4c)

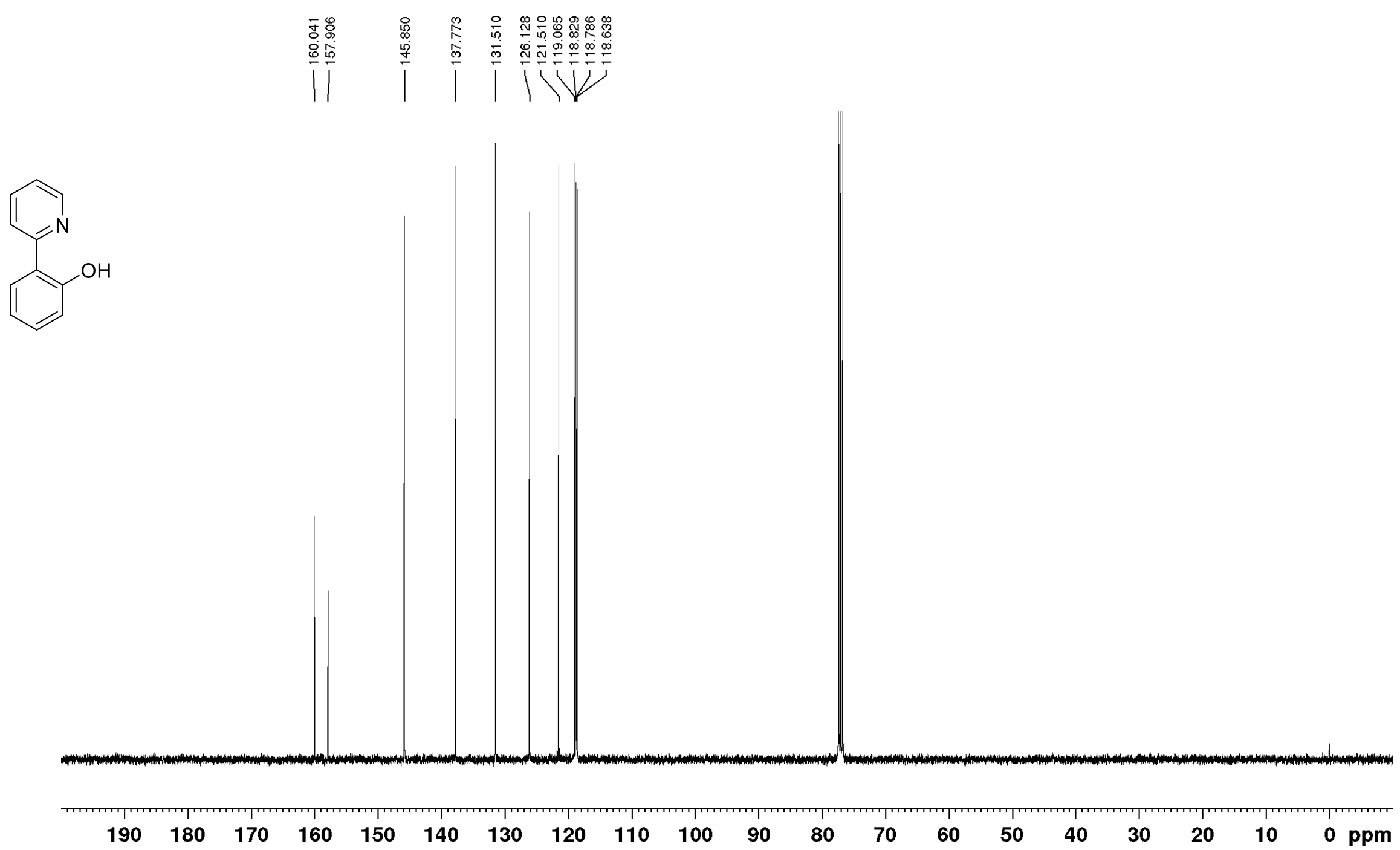


${ }^{1} \mathrm{H}$ NMR (CDCl 3 , $\left.400 \mathrm{MHz}\right)$ of 2-(3-Methylpyridin-2-yl)phenol (4d)

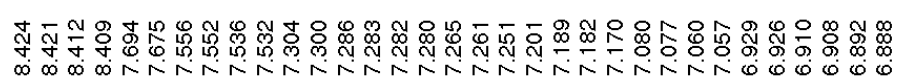

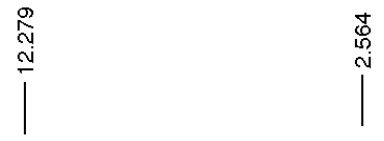

OH
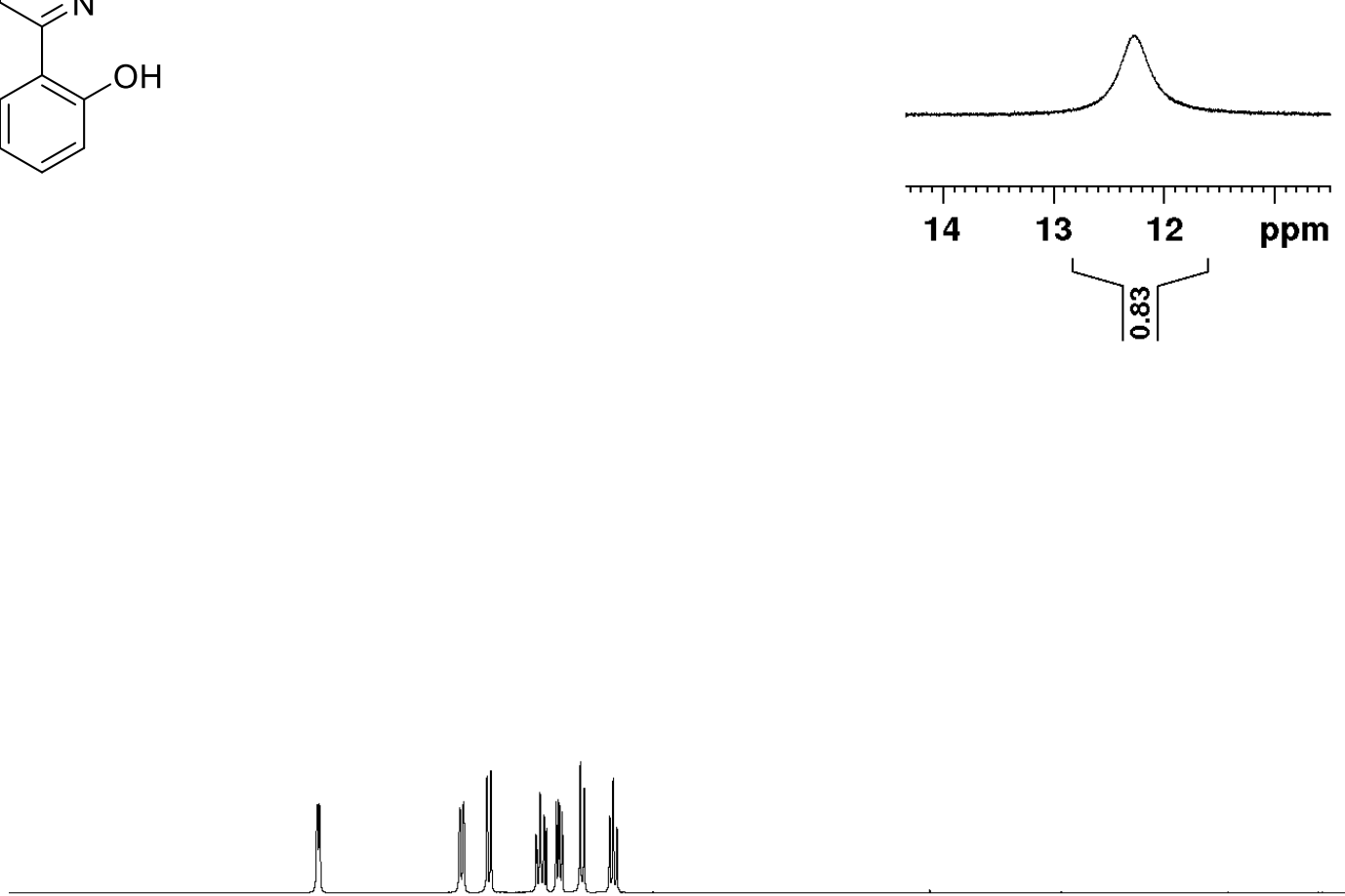

9

8

|

5

$\left|\frac{0}{m}\right|$ 
${ }^{13} \mathrm{C}$ NMR $\left(\mathrm{CDCl}_{3}, 100 \mathrm{MHz}\right)$ of 2-(3-Methylpyridin-2-yl)phenol (4d)
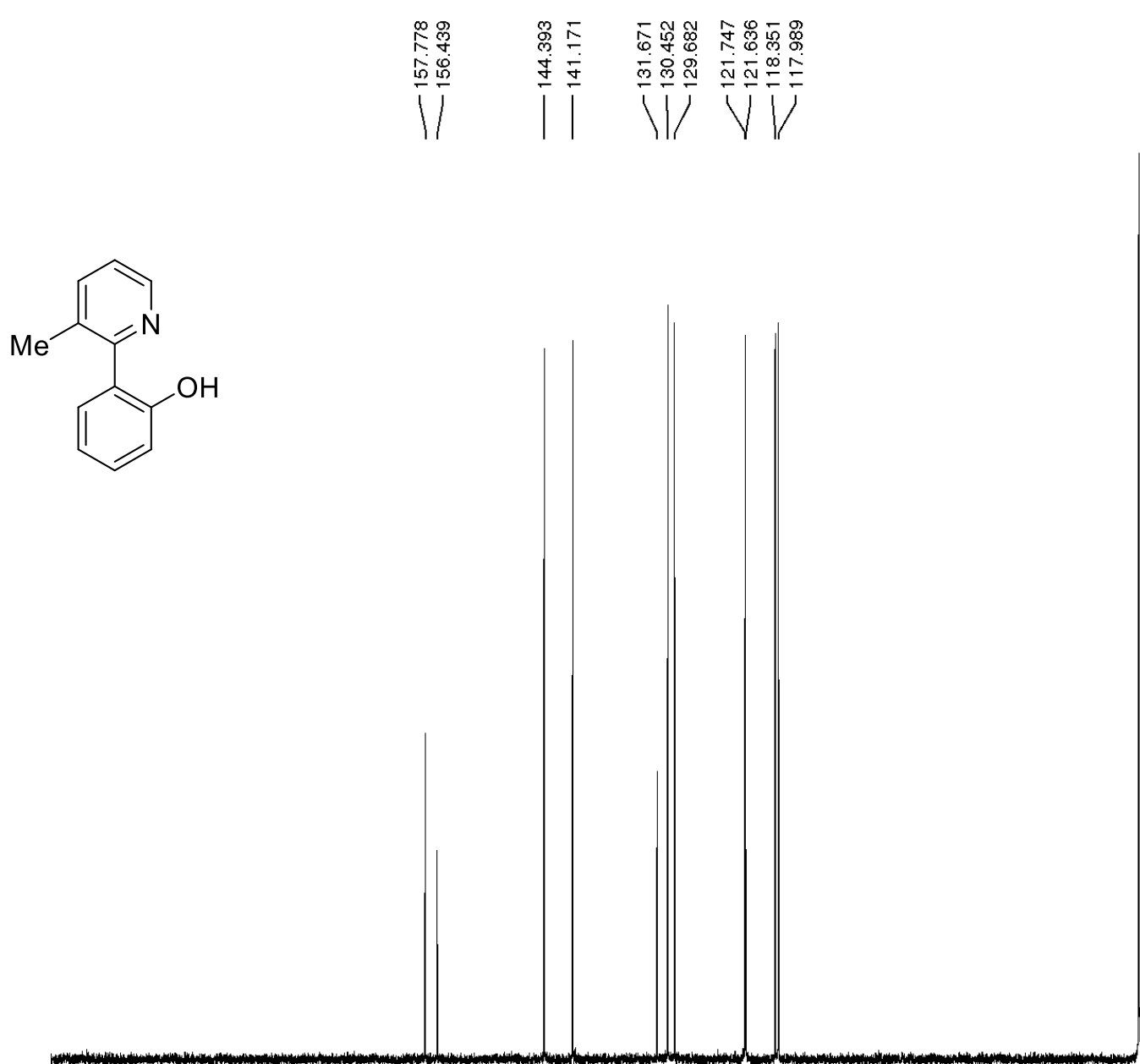

$\begin{array}{llllllllll}190 & 180 & 170 & 160 & 150 & 140 & 130 & 120 & 110 & 100\end{array}$

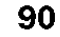

80

$70 \quad 60$ 
${ }^{1} \mathrm{H}$ NMR (CDCl 3 , $\left.400 \mathrm{MHz}\right)$ of 2-(6-Methylpyridin-2-yl)phenol (4e)
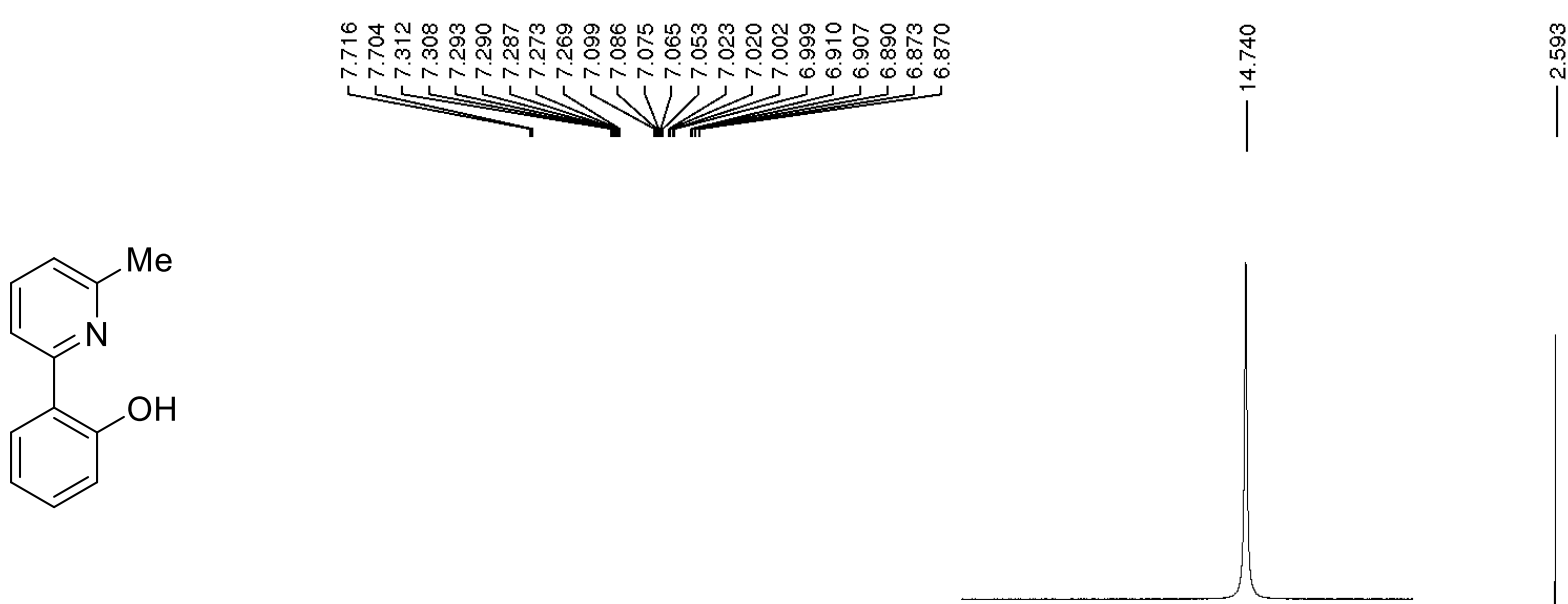

|

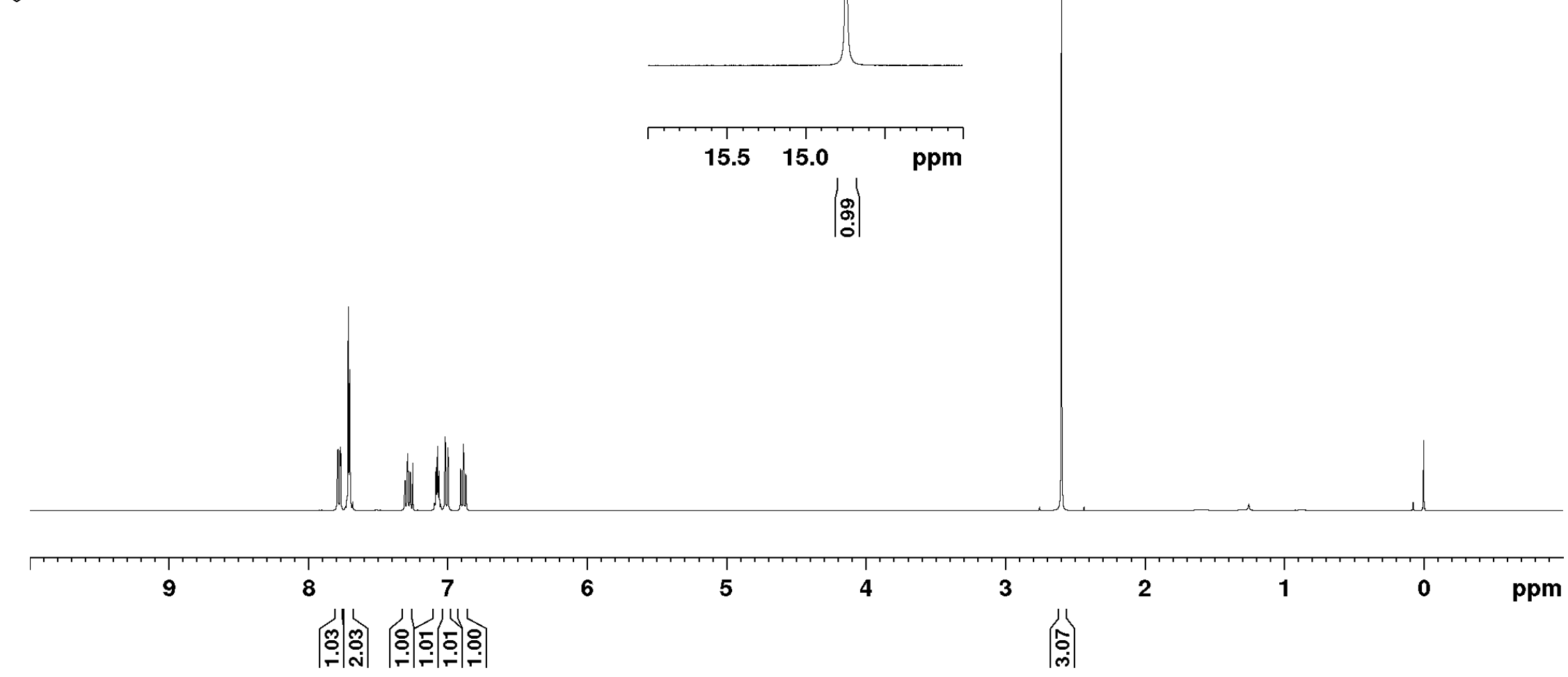


${ }^{13} \mathrm{C} \mathrm{NMR}\left(\mathrm{CDCl}_{3}, 100 \mathrm{MHz}\right)$ of 2-(6-Methylpyridin-2-yl)phenol (4e)

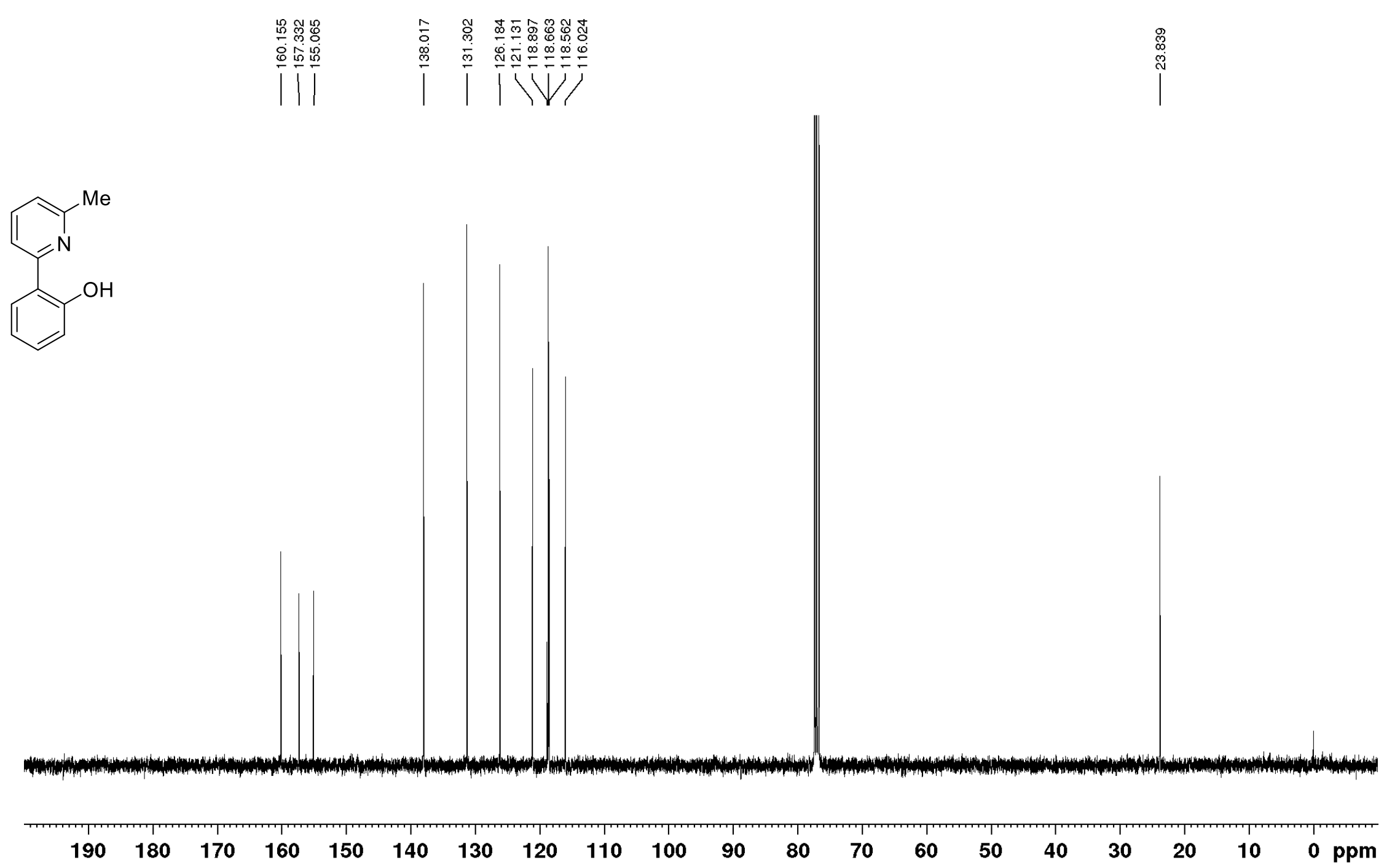


${ }^{1} \mathrm{H}$ NMR $\left(\mathrm{CDCl}_{3}, 400 \mathrm{MHz}\right)$ of 2-(Pyridin-2-yloxy)phenol (4f)

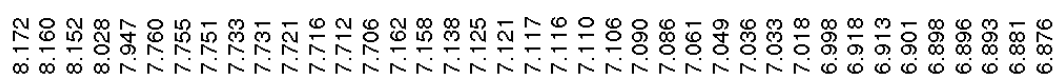

(N)
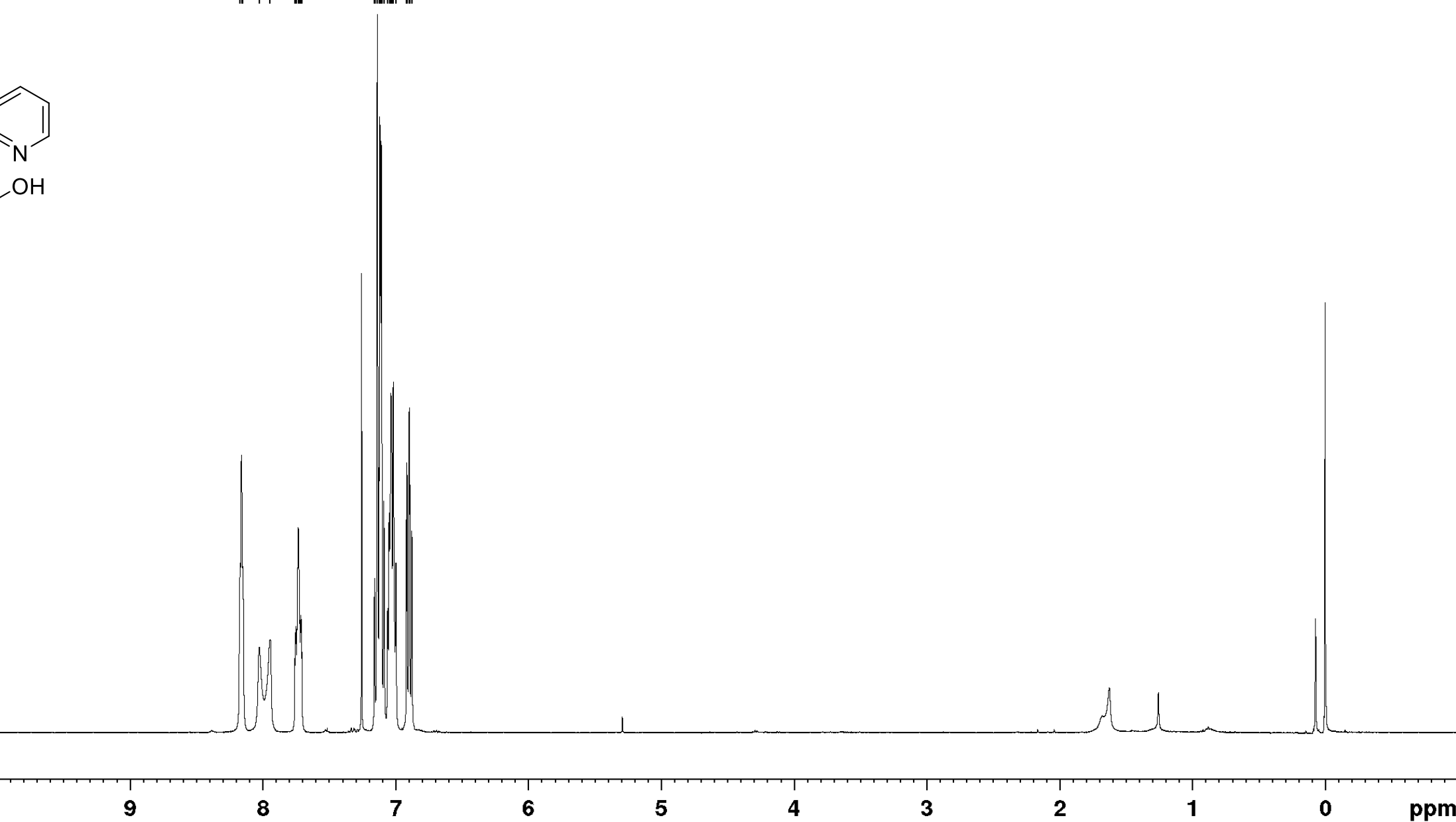

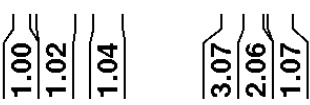


${ }^{13} \mathrm{C} \mathrm{NMR}\left(\mathrm{CDCl}_{3}, 100 \mathrm{MHz}\right)$ of 2-(Pyridin-2-yloxy)phenol (4f)
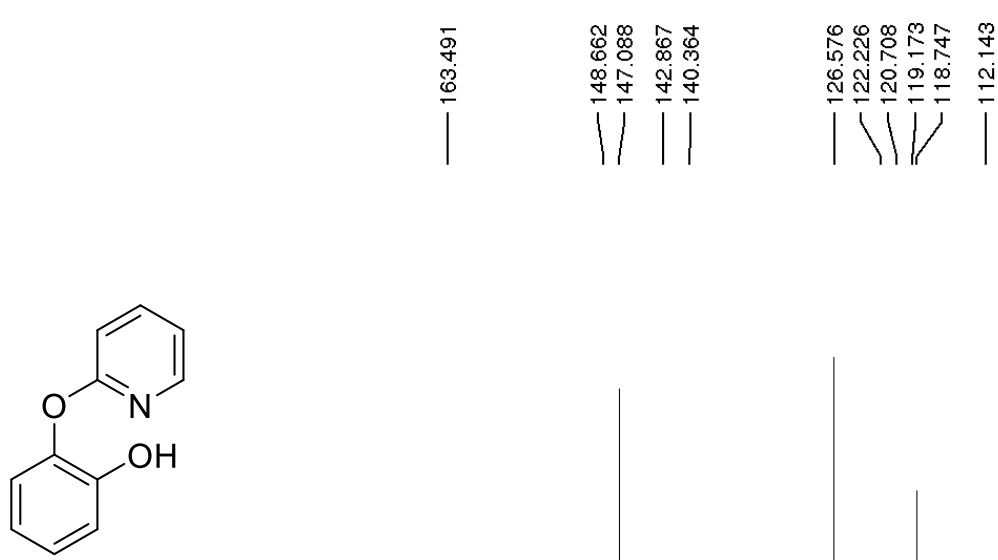

II
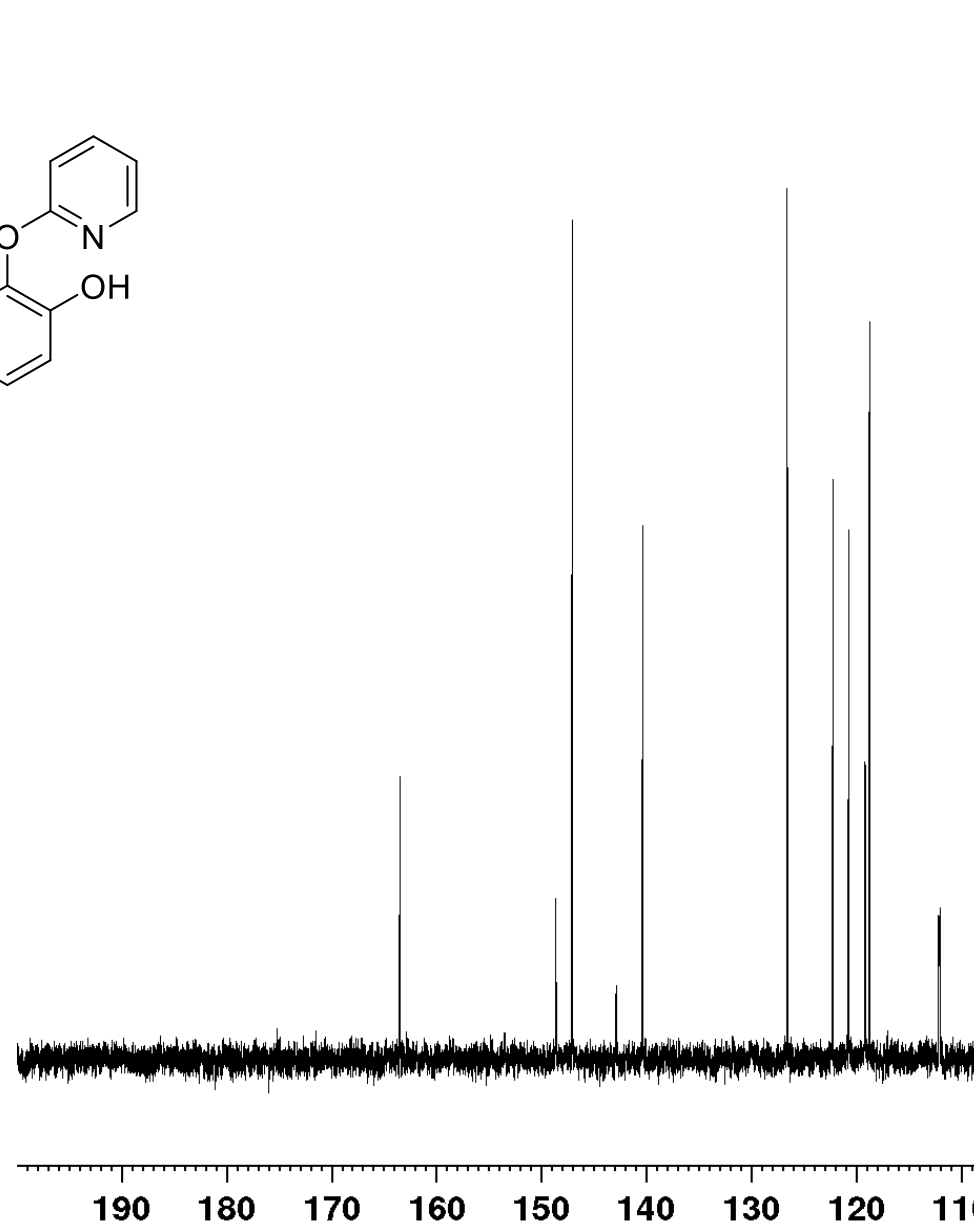

140130

$\begin{array}{lll}120 & 110 & 100\end{array}$

$90 \quad 80$

$70 \quad 60$

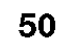

40

3020

10

0 ppm 
${ }^{1} \mathrm{H}$ NMR $\left(\mathrm{CDCl}_{3}, 400 \mathrm{MHz}\right)$ of 2-(Pyridin-2-ylmethyl)phenol (4g)
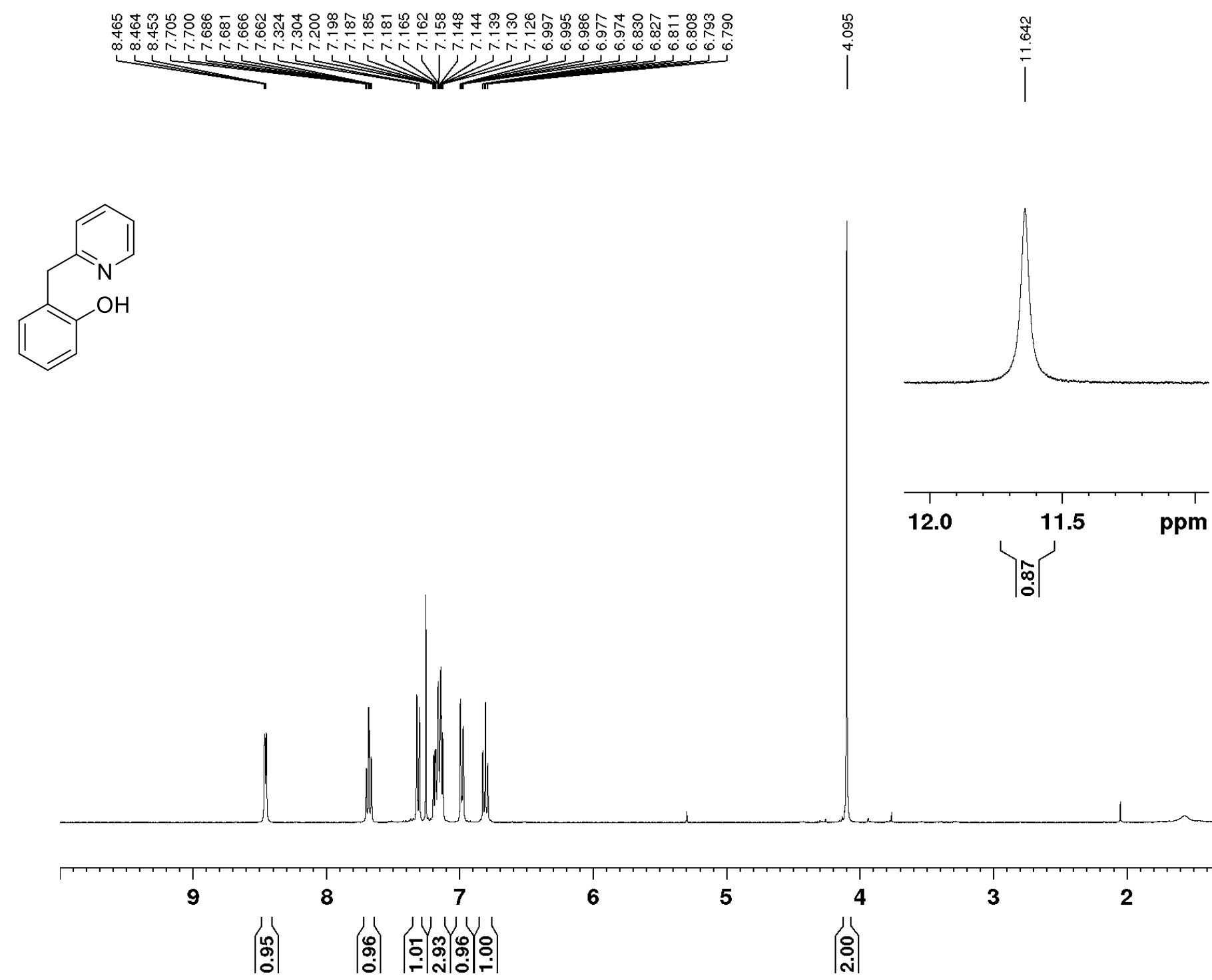
${ }^{13} \mathrm{C}$ NMR ( $\left.\mathrm{CDCl}_{3}, 100 \mathrm{MHz}\right)$ of 2-(Pyridin-2-ylmethyl)phenol (4g)
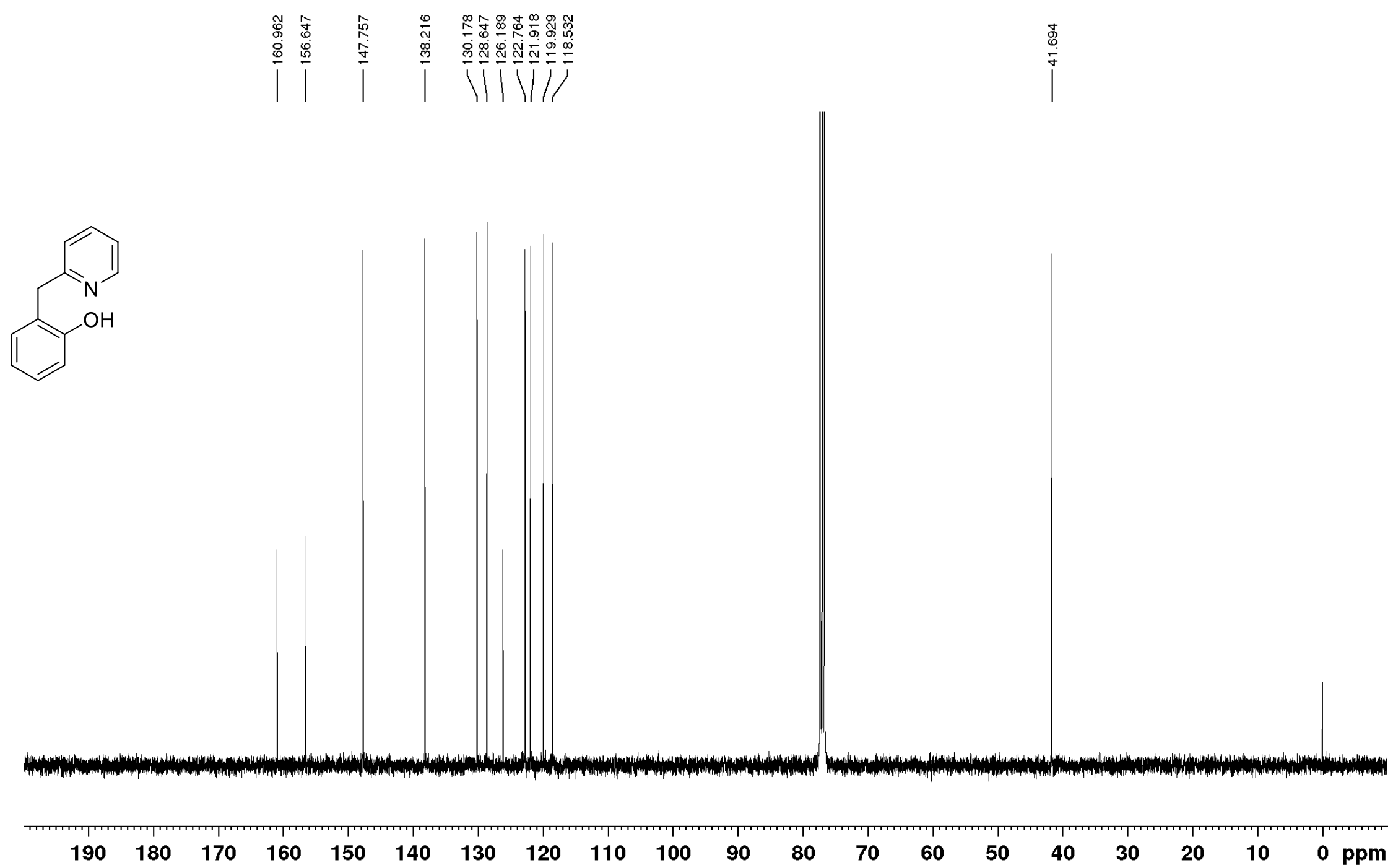
${ }^{1} \mathrm{H}$ NMR $\left(\mathrm{CDCl}_{3}, 400 \mathrm{MHz}\right)$ of 2-[(1H-Pyrazol-1-yl)methyl]phenol (4h)

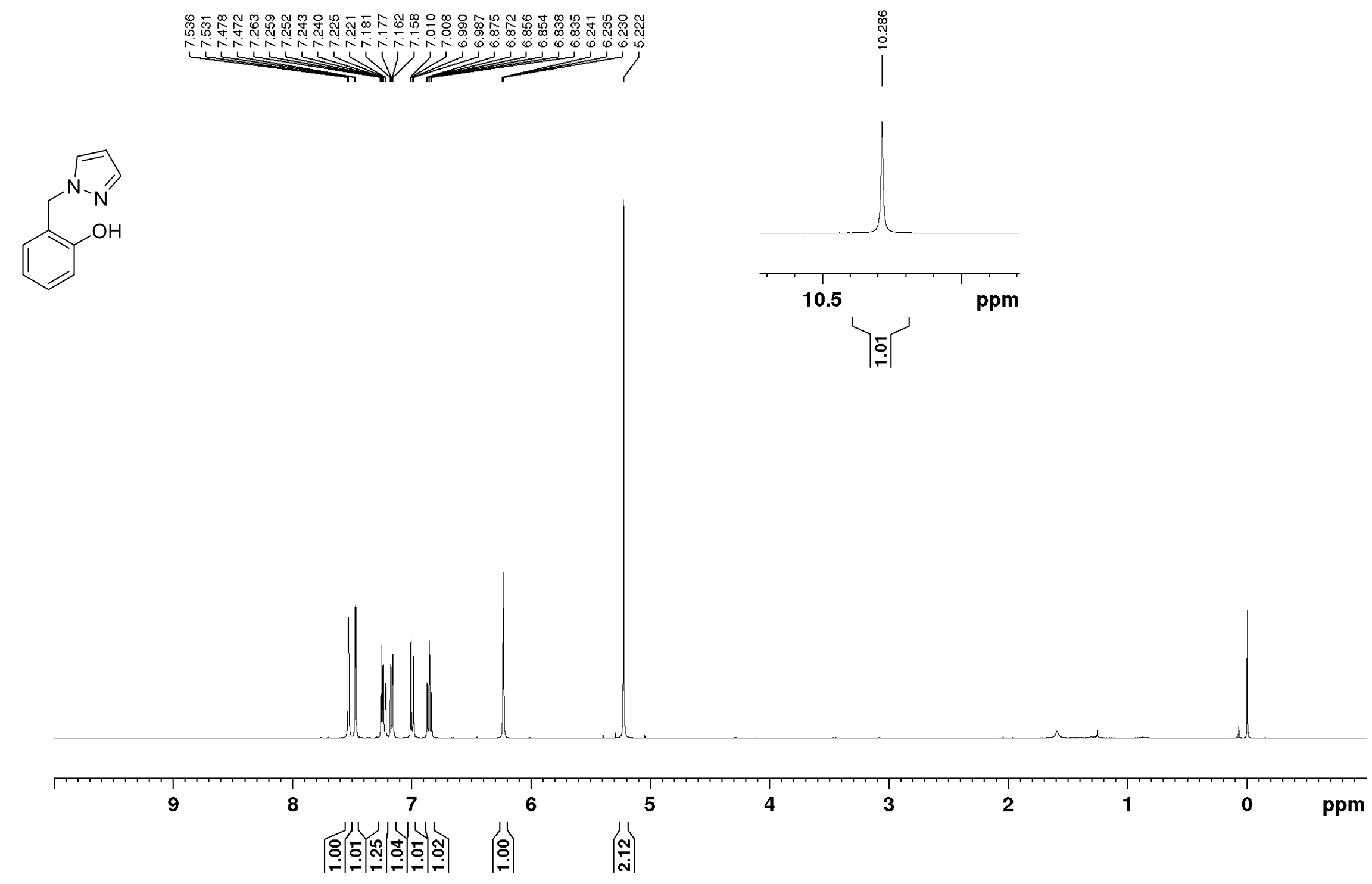


${ }^{13} \mathrm{C} \mathrm{NMR}\left(\mathrm{CDCl}_{3}, 100 \mathrm{MHz}\right)$ of 2-[(1H-Pyrazol-1-yl)methyl]phenol (4h)

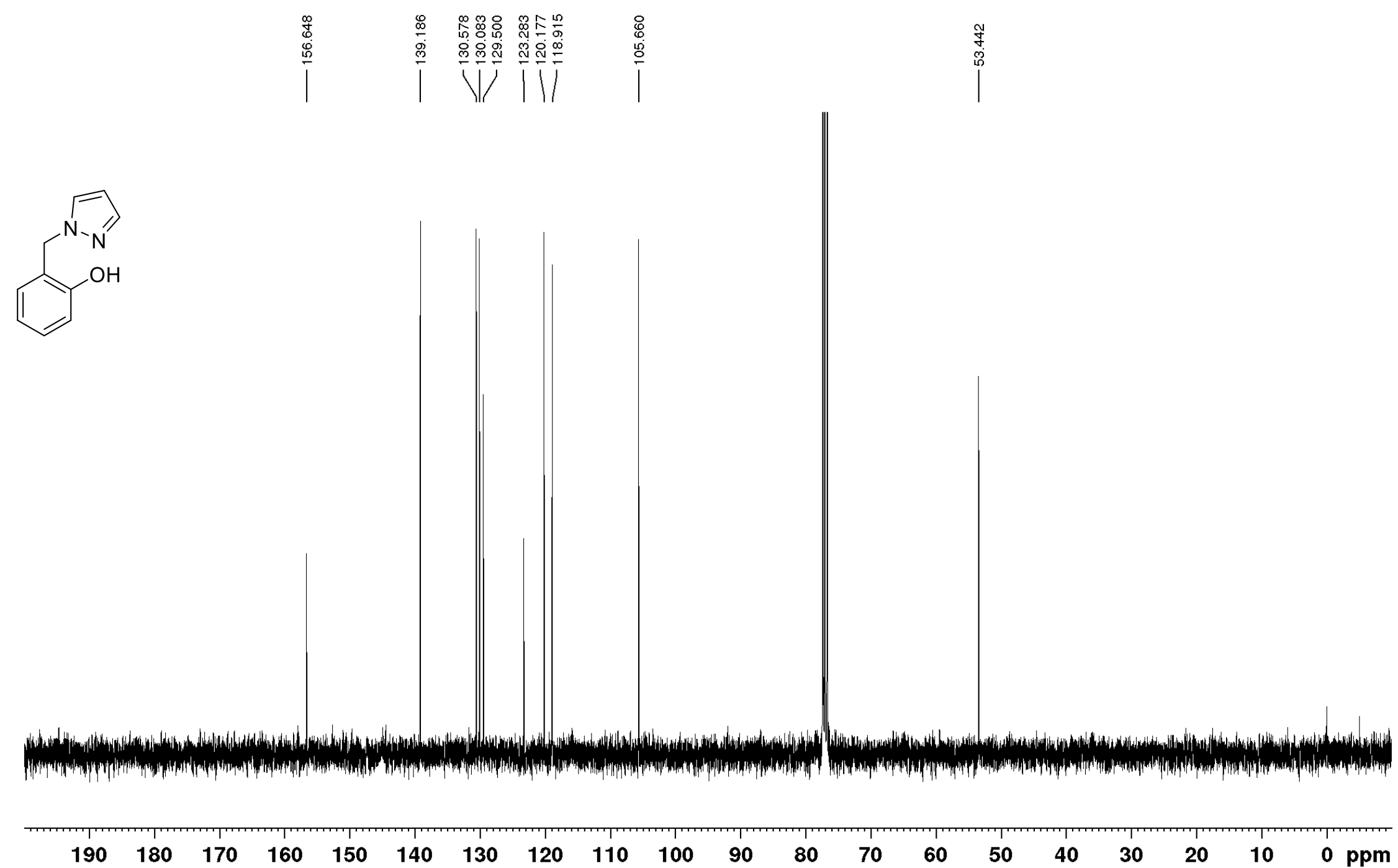


${ }^{1} \mathrm{H}$ NMR $\left(\mathrm{CDCl}_{3}, 400 \mathrm{MHz}\right)$ of 2-(Isoquinolin-1-yl)phenol (4i)

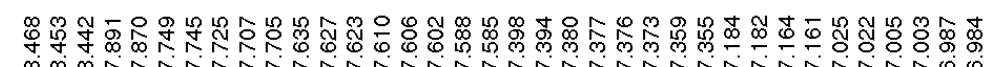

$\longrightarrow$ -

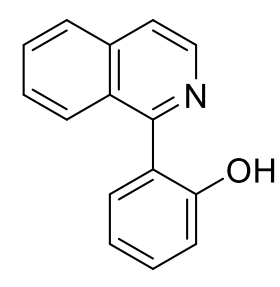

7

6

5

4

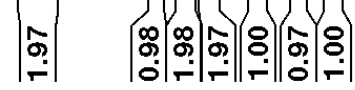


${ }^{13} \mathrm{C}$ NMR $\left(\mathrm{CDCl}_{3}, 100 \mathrm{MHz}\right)$ of 2-(Isoquinolin-1-yl)phenol (4i)
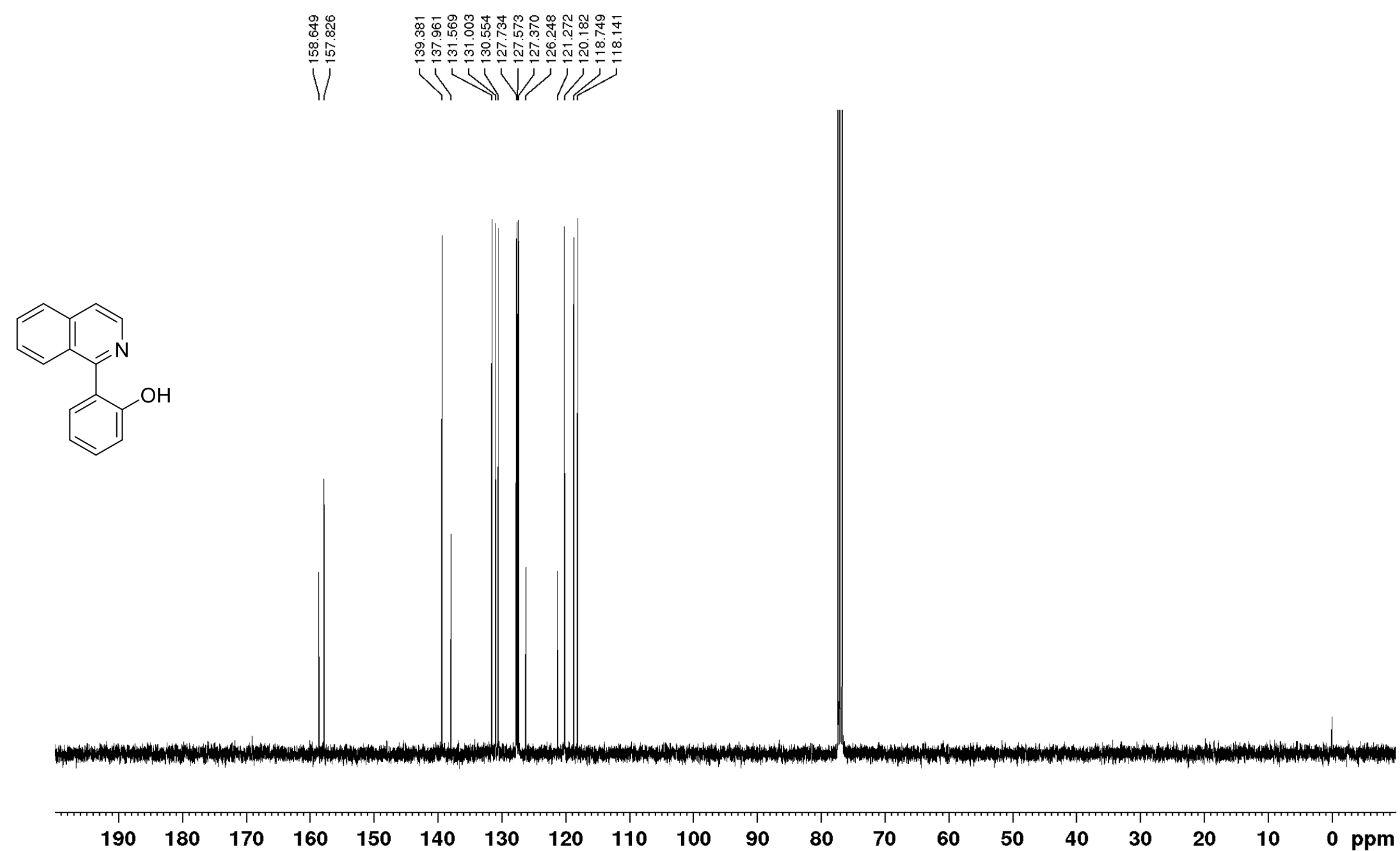
${ }^{1} \mathrm{H}$ NMR $\left(\mathrm{CDCl}_{3}, 400 \mathrm{MHz}\right)$ of Benzo[ $\left.h\right]$ quinolin-10-ol (4j)
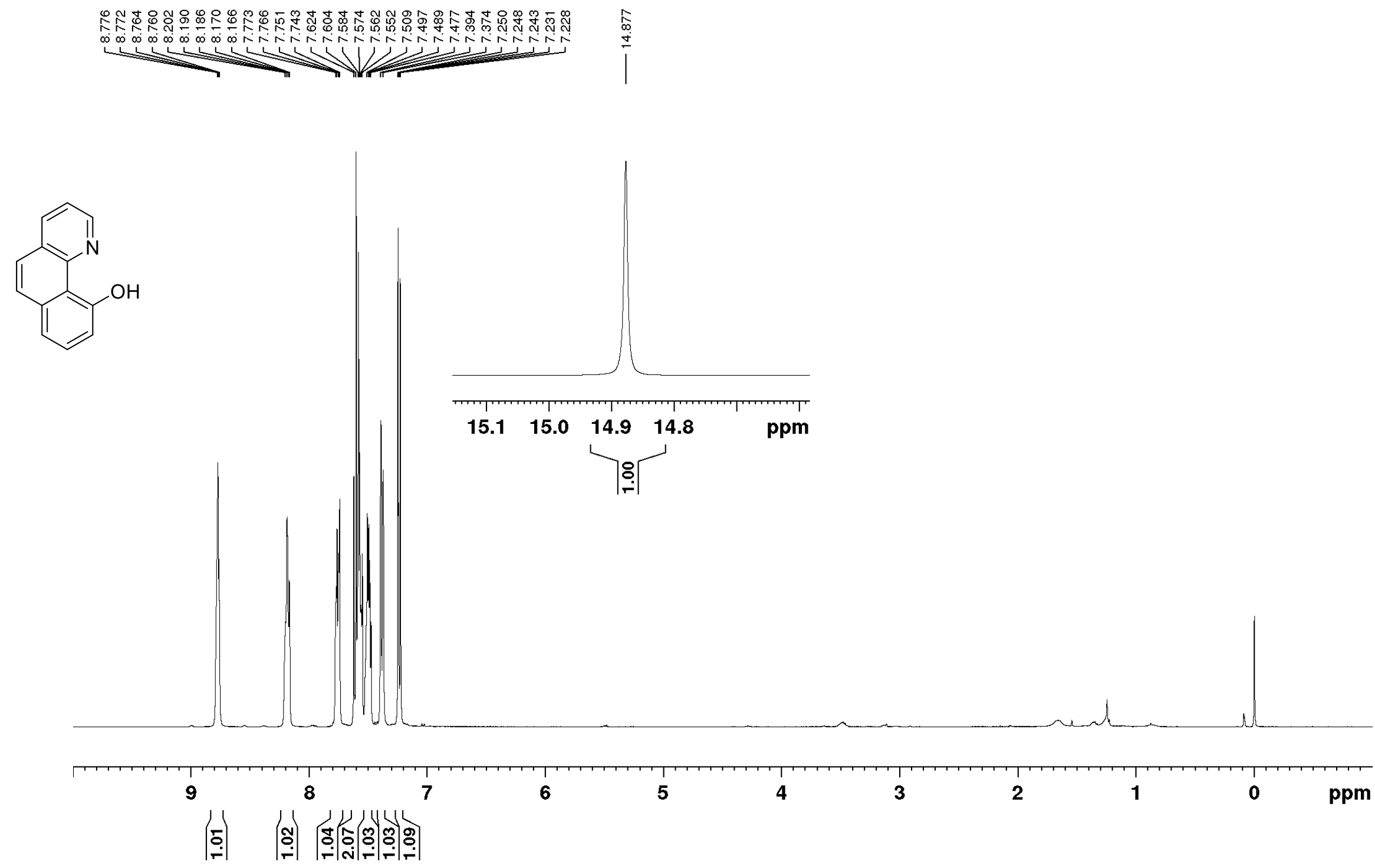
${ }^{13} \mathrm{C}$ NMR $\left(\mathrm{CDCl}_{3}, 100 \mathrm{MHz}\right)$ of Benzo[h]quinolin-10-ol (4j)
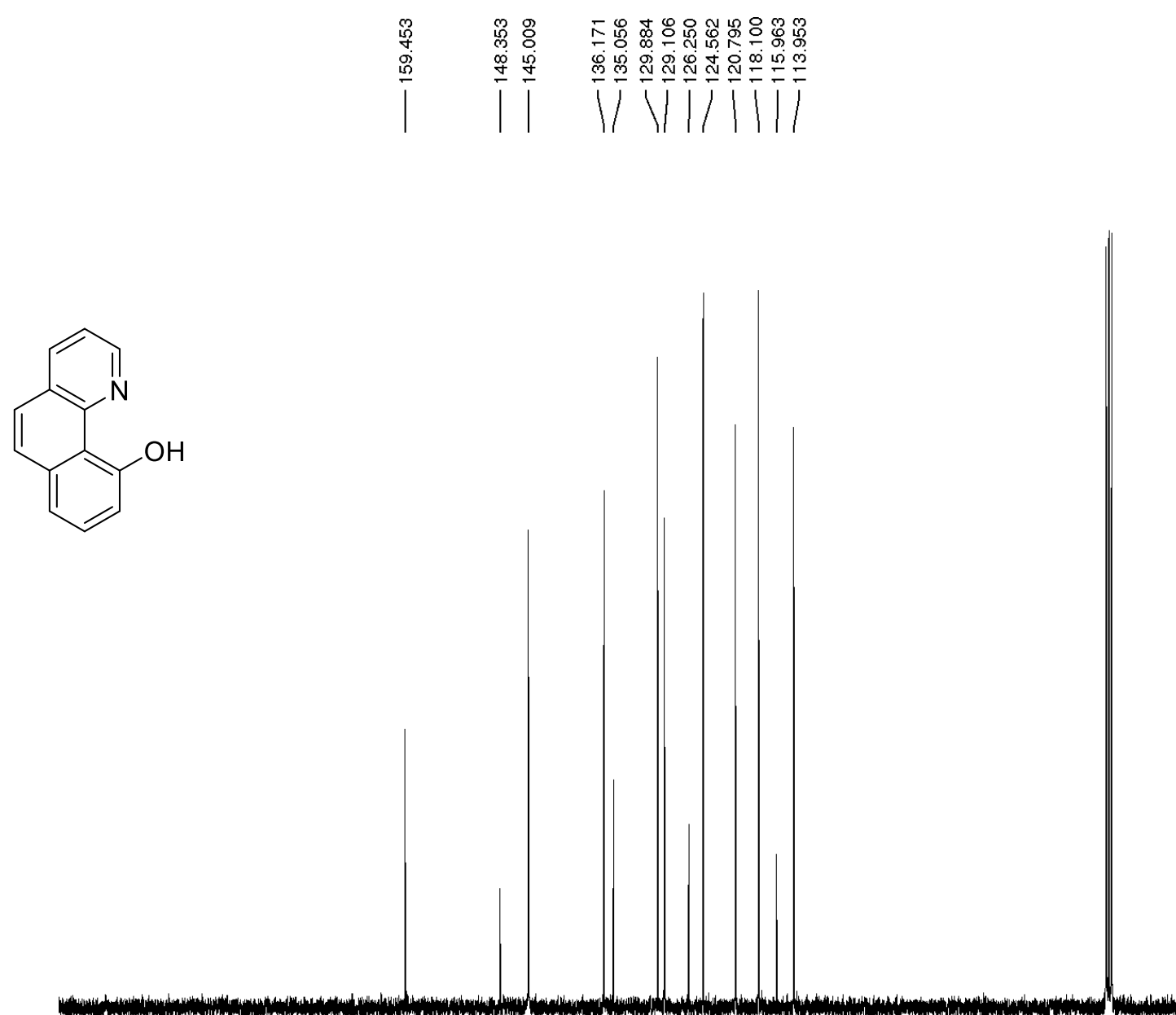

$\begin{array}{llllllllll}190 & 180 & 170 & 160 & 150 & 140 & 130 & 120 & 110 & 100\end{array}$

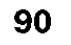

80

7060 
${ }^{1} \mathrm{H}$ NMR $\left(\mathrm{CDCl}_{3}, 400 \mathrm{MHz}\right)$ of 2-[(Dimethylamino)methyl]phenol (4k)
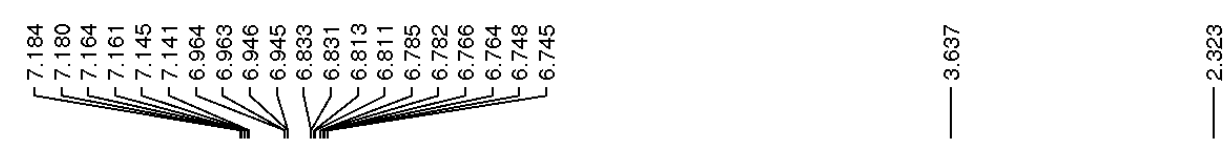

$\mathrm{NOH}^{\mathrm{NMe}_{2}}$

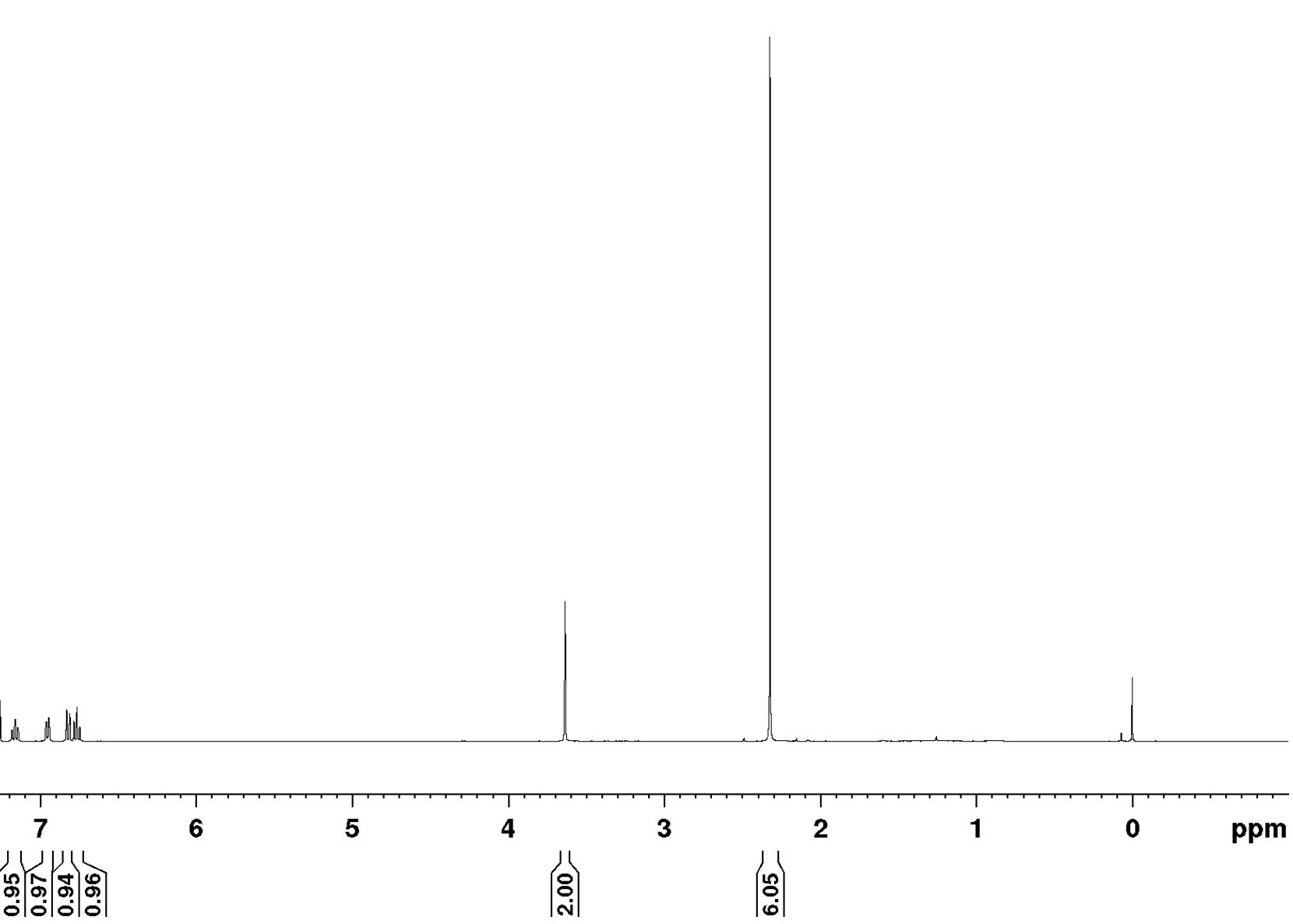


${ }^{13} \mathrm{C} \mathrm{NMR}\left(\mathrm{CDCl}_{3}, 100 \mathrm{MHz}\right)$ of 2-[(Dimethylamino)methyl]phenol (4k)

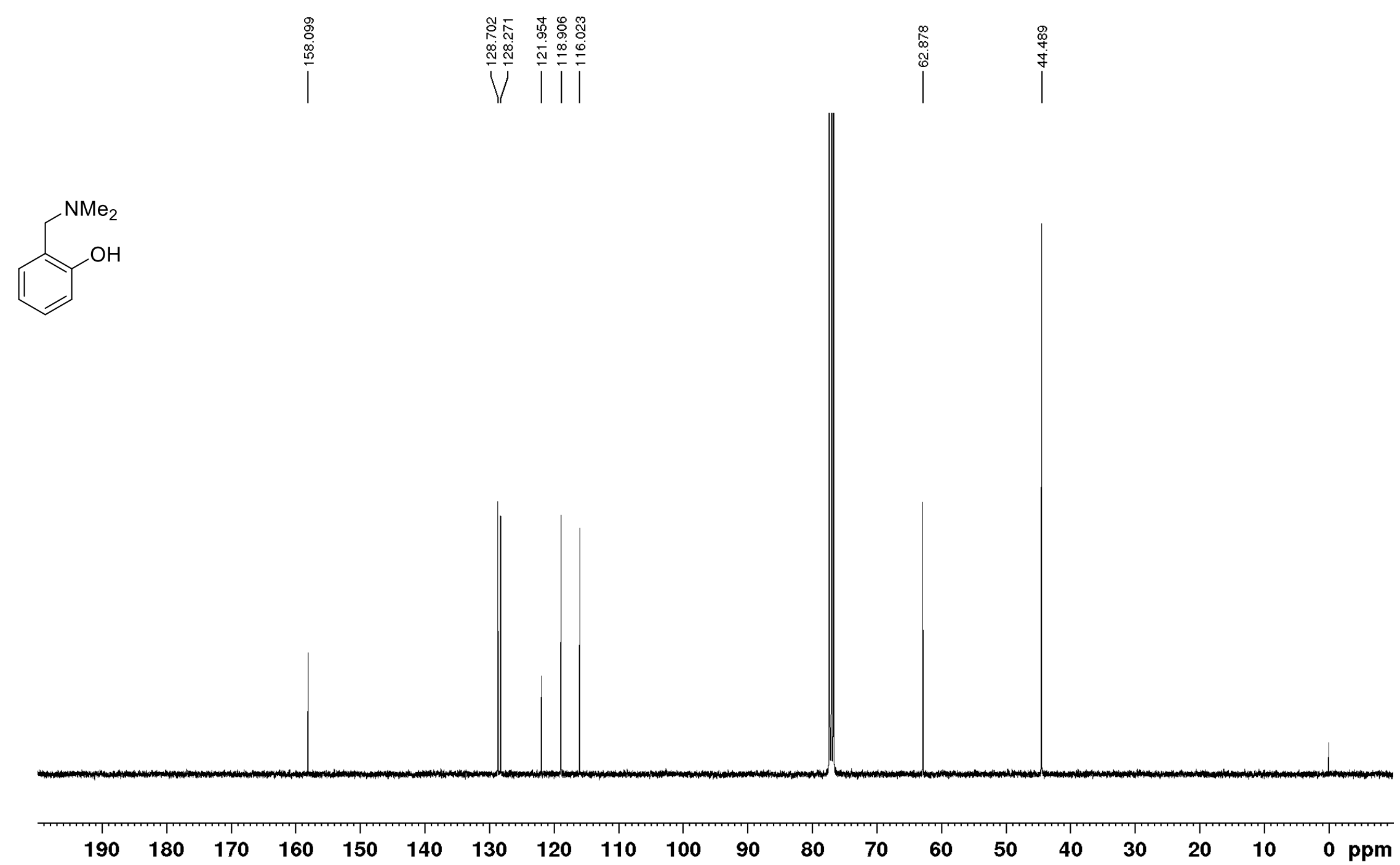


${ }^{1} \mathrm{H}$ NMR $\left(\mathrm{CDCl}_{3}, 400 \mathrm{MHz}\right)$ of 2-\{[Benzyl(methyl)amino]methyl $\}$ phenol (4I)
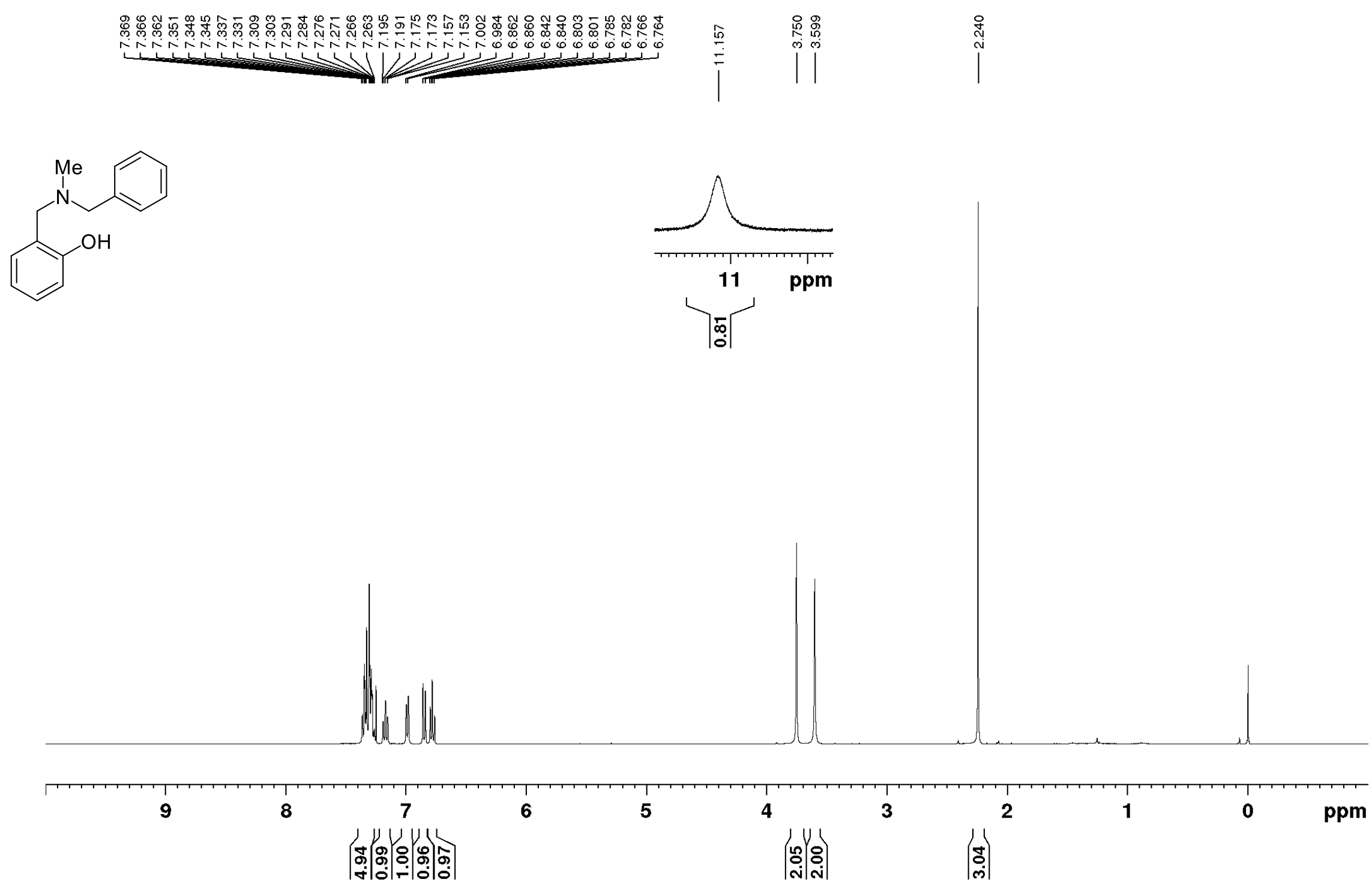
${ }^{13} \mathrm{C}$ NMR $\left(\mathrm{CDCl}_{3}, 100 \mathrm{MHz}\right)$ of 2-\{[Benzyl(methyl)amino]methyl $\}$ phenol (4I)

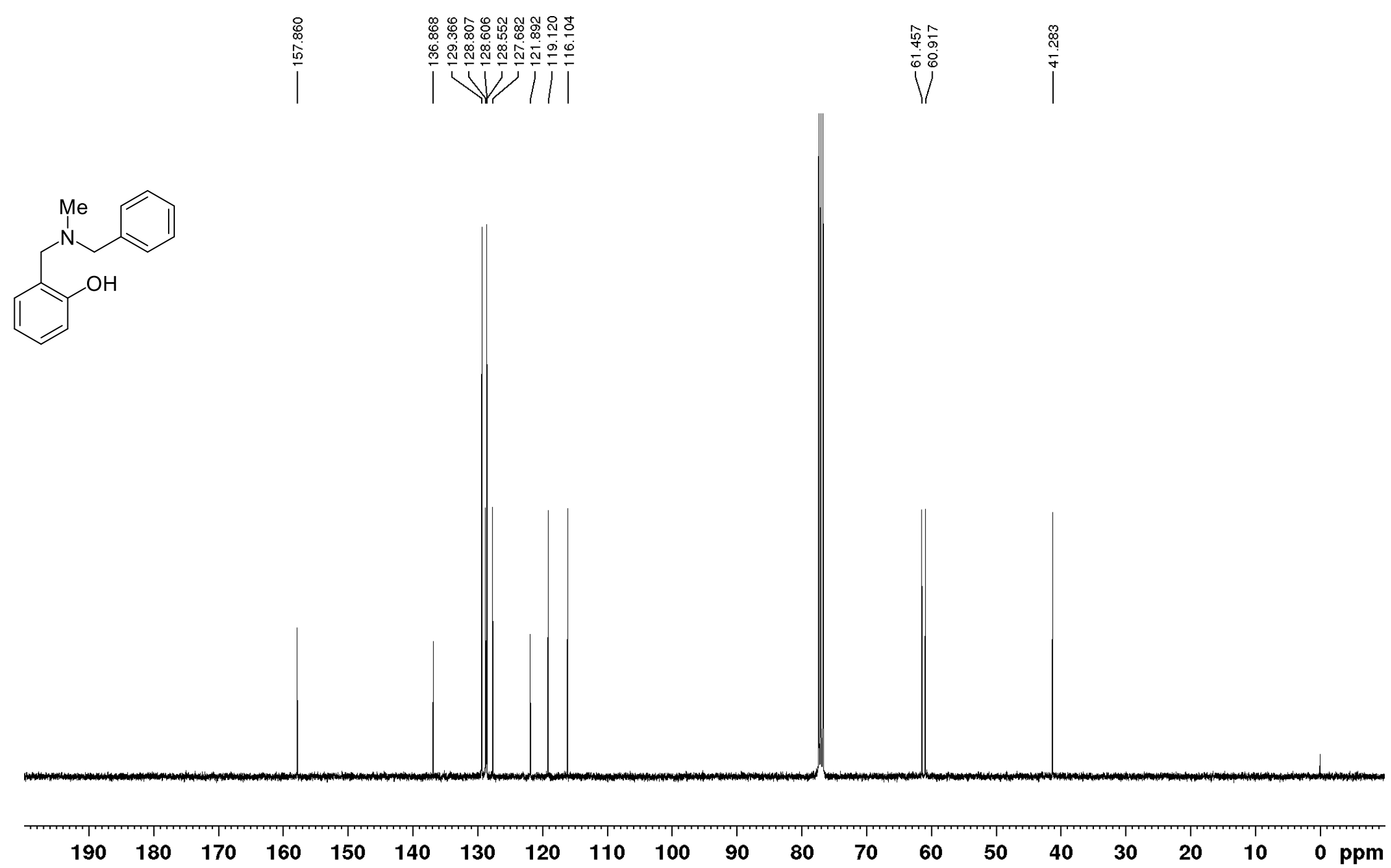


${ }^{1} \mathrm{H}$ NMR $\left(\mathrm{CDCl}_{3}, 400 \mathrm{MHz}\right)$ of 3-Methyl-2-(1H-pyrazol-1-yl)phenol (4n)
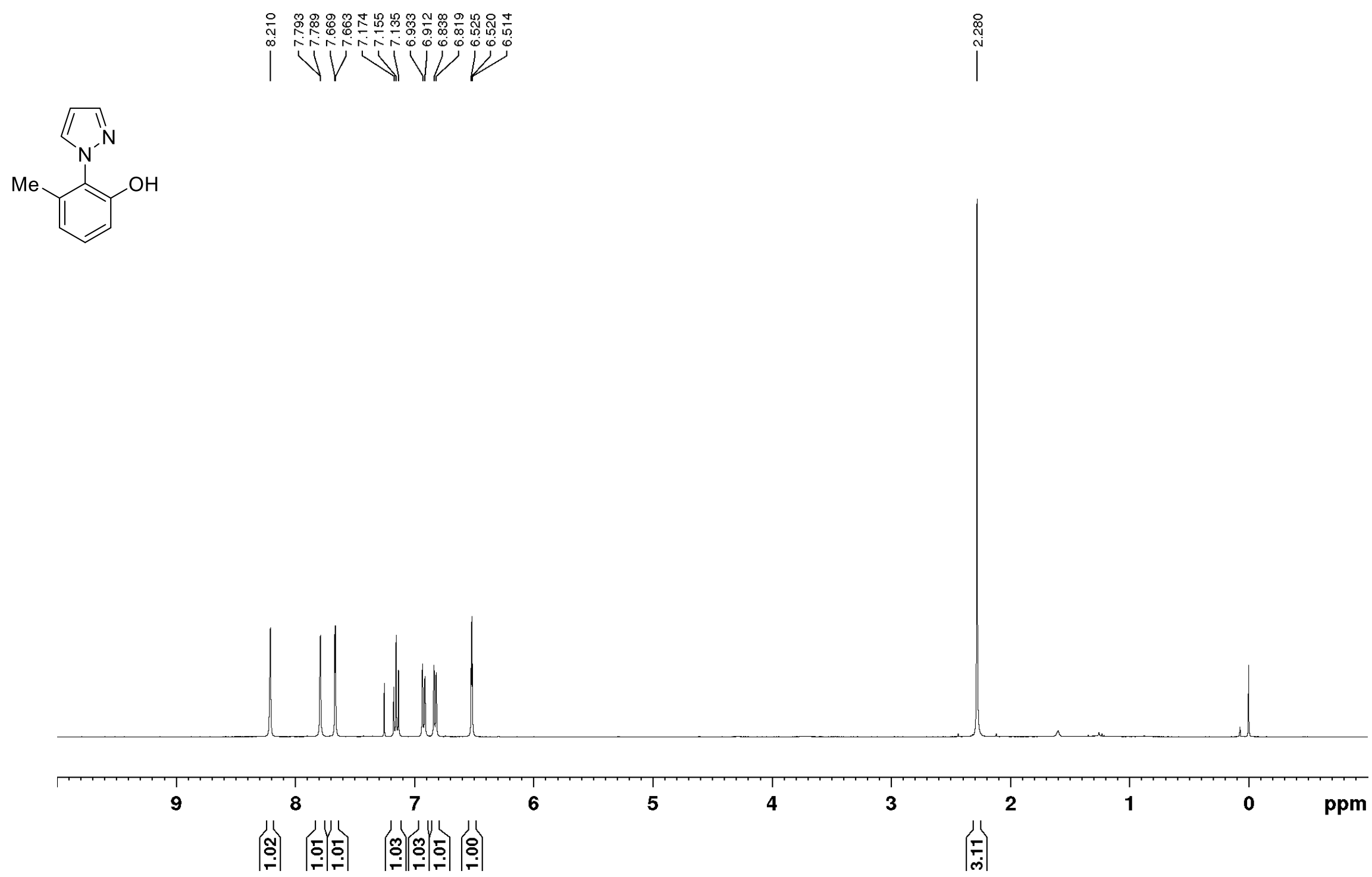
${ }^{13} \mathrm{C}$ NMR $\left(\mathrm{CDCl}_{3}, 100 \mathrm{MHz}\right)$ of 3-Methyl-2-(1H-pyrazol-1-yl)phenol (4n)

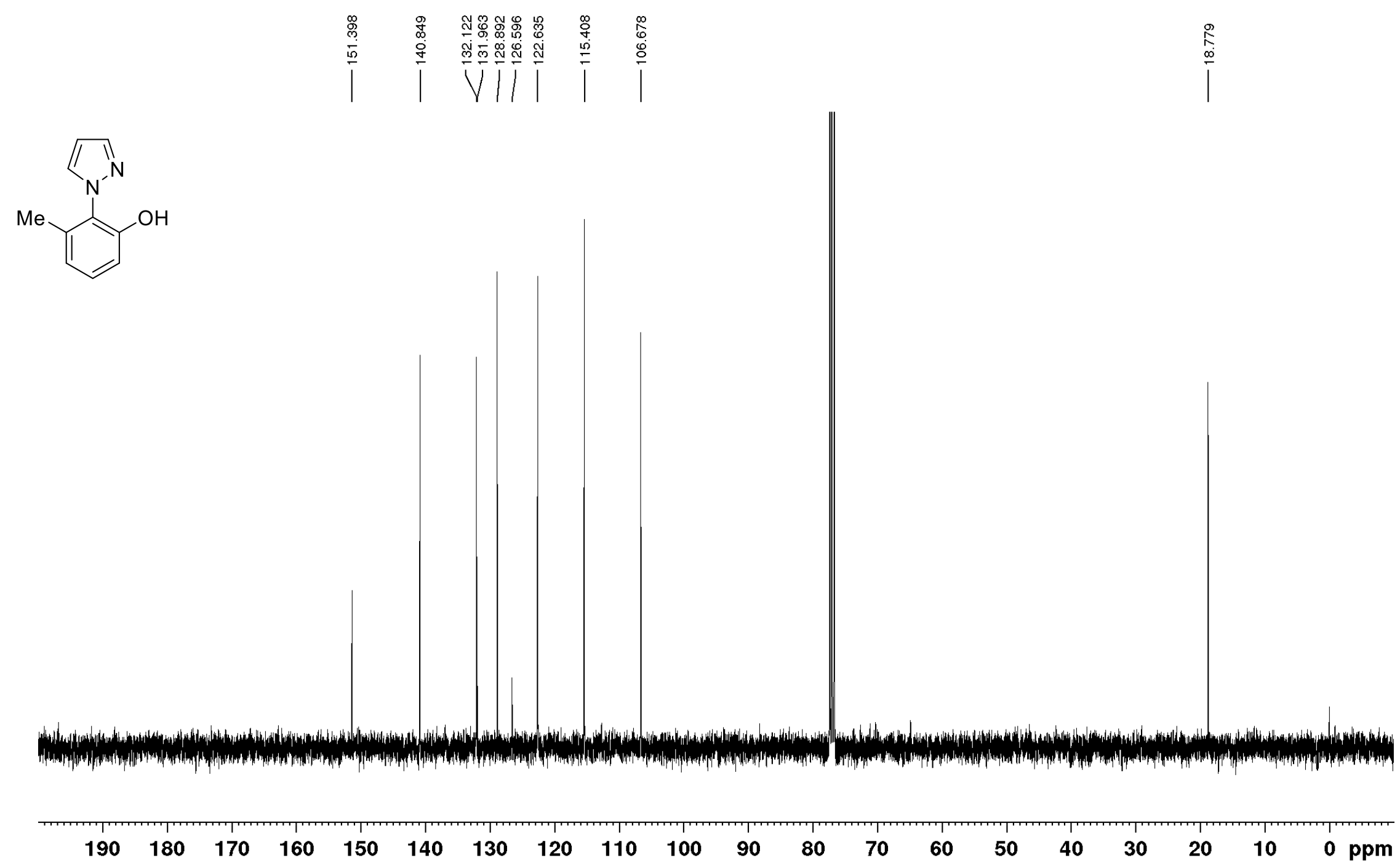


${ }^{1} \mathrm{H}$ NMR $\left(\mathrm{CDCl}_{3}, 400 \mathrm{MHz}\right)$ of 1-[5-Methyl-2-(4,4,5,5-tetramethyl-1,3,2-dioxaborolan-2-yl)phenyl]-1H-pyrazole (3o)

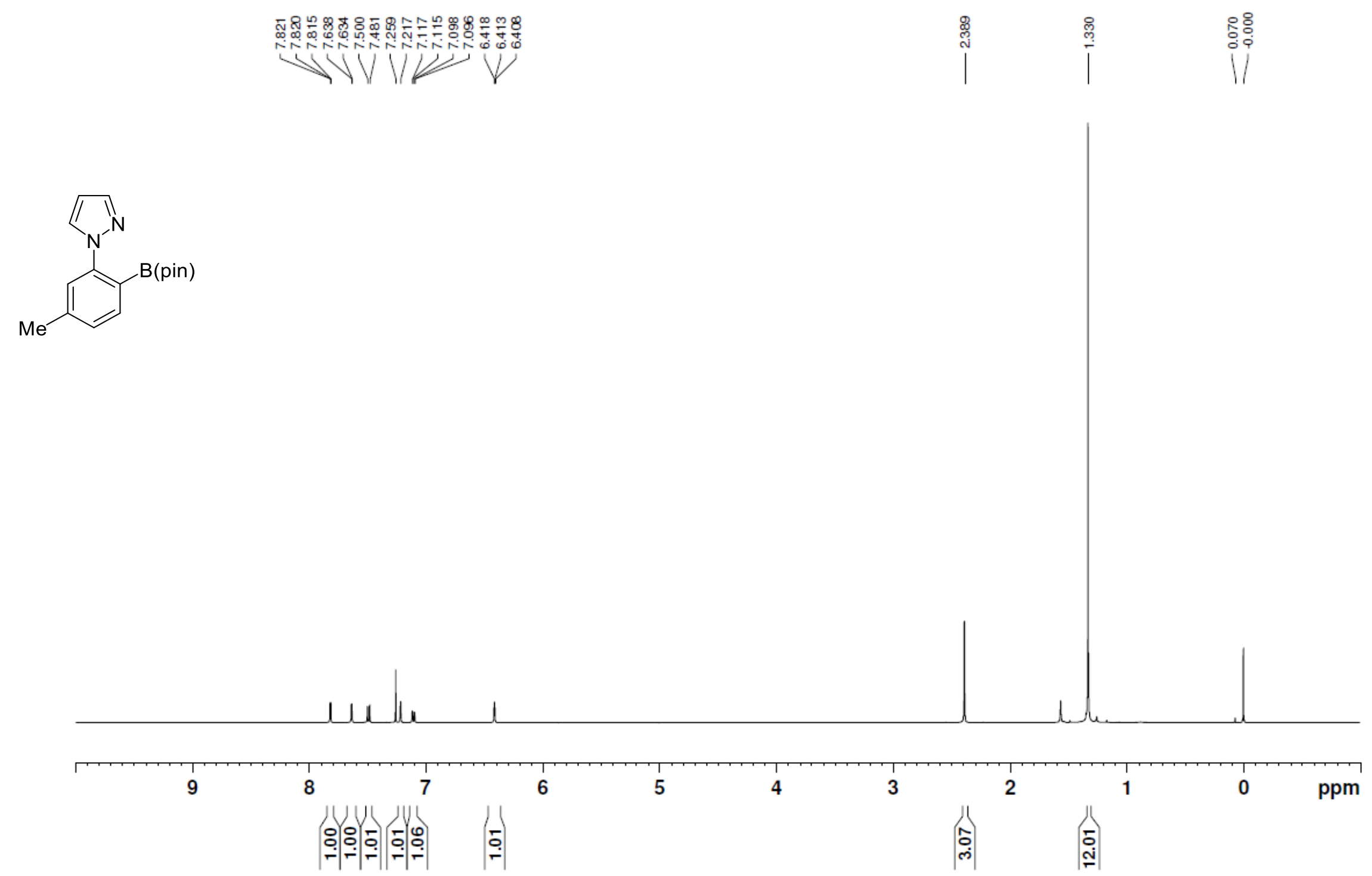


${ }^{13} \mathrm{C}$ NMR $\left(\mathrm{CDCl}_{3}, 100 \mathrm{MHz}\right)$ of 1-[5-Methyl-2-(4,4,5,5-tetramethyl-1,3,2-dioxaborolan-2-yl)phenyl]-1H-pyrazole (3o)
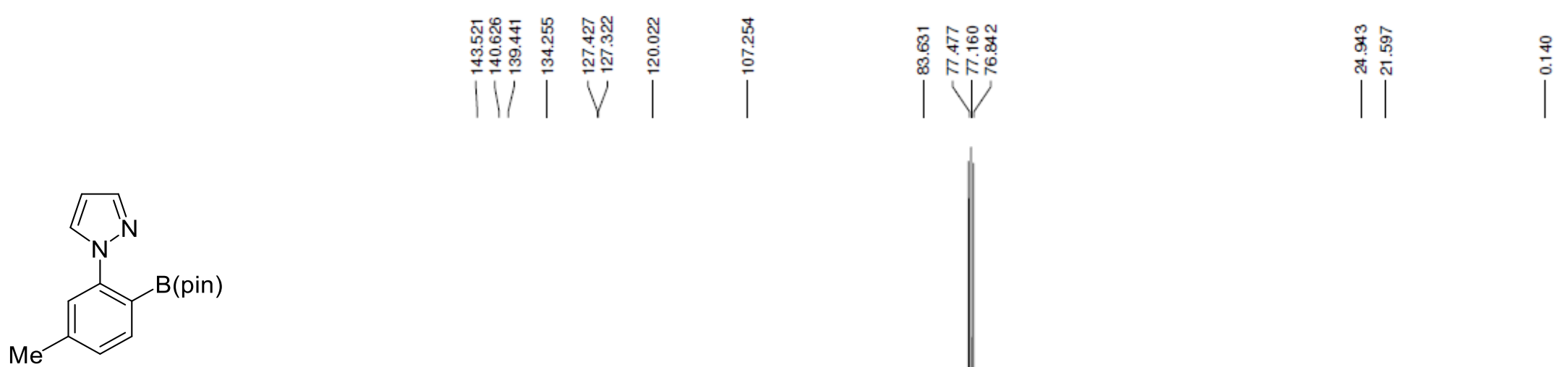

\section{(1)}

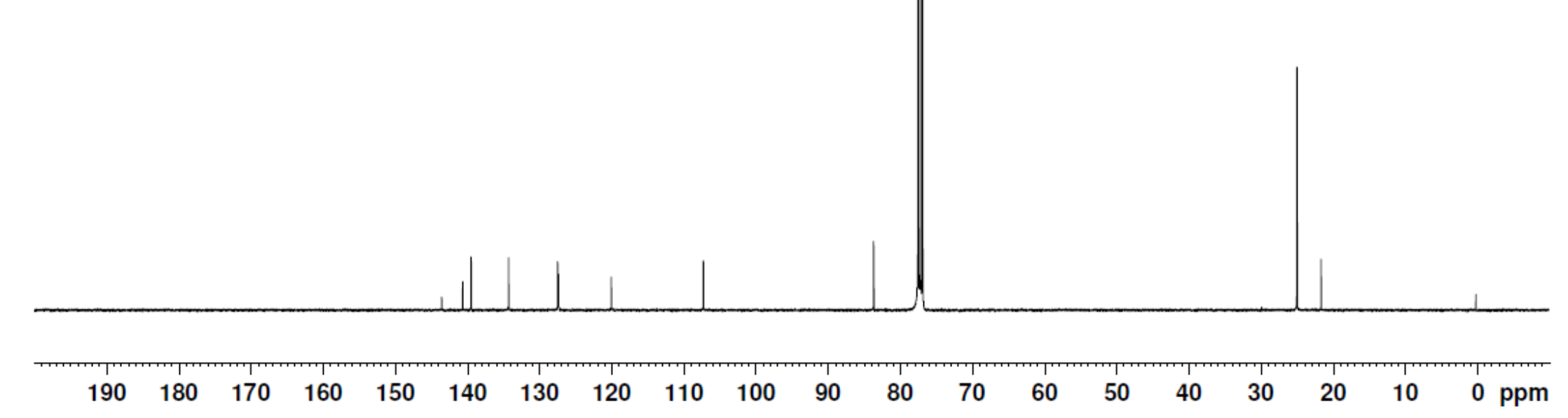


${ }^{1} \mathrm{H}$ NMR $\left(\mathrm{CDCl}_{3}, 400 \mathrm{MHz}\right)$ of 4-Methyl-2-(1H-pyrazol-1-yl)phenol (4o)
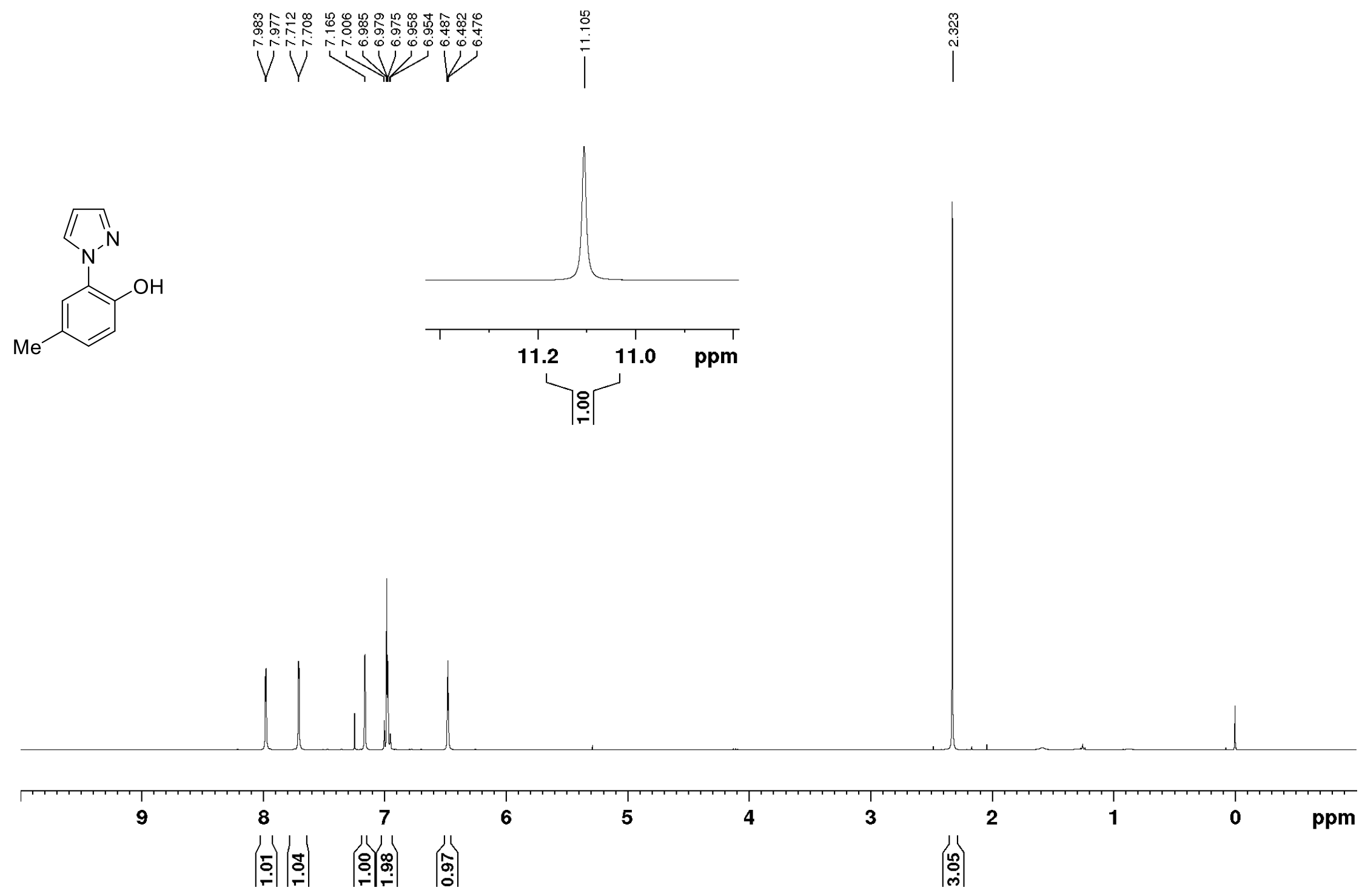
${ }^{13} \mathrm{C}$ NMR $\left(\mathrm{CDCl}_{3}, 100 \mathrm{MHz}\right)$ of 4-Methyl-2-(1H-pyrazol-1-yl)phenol (4o)

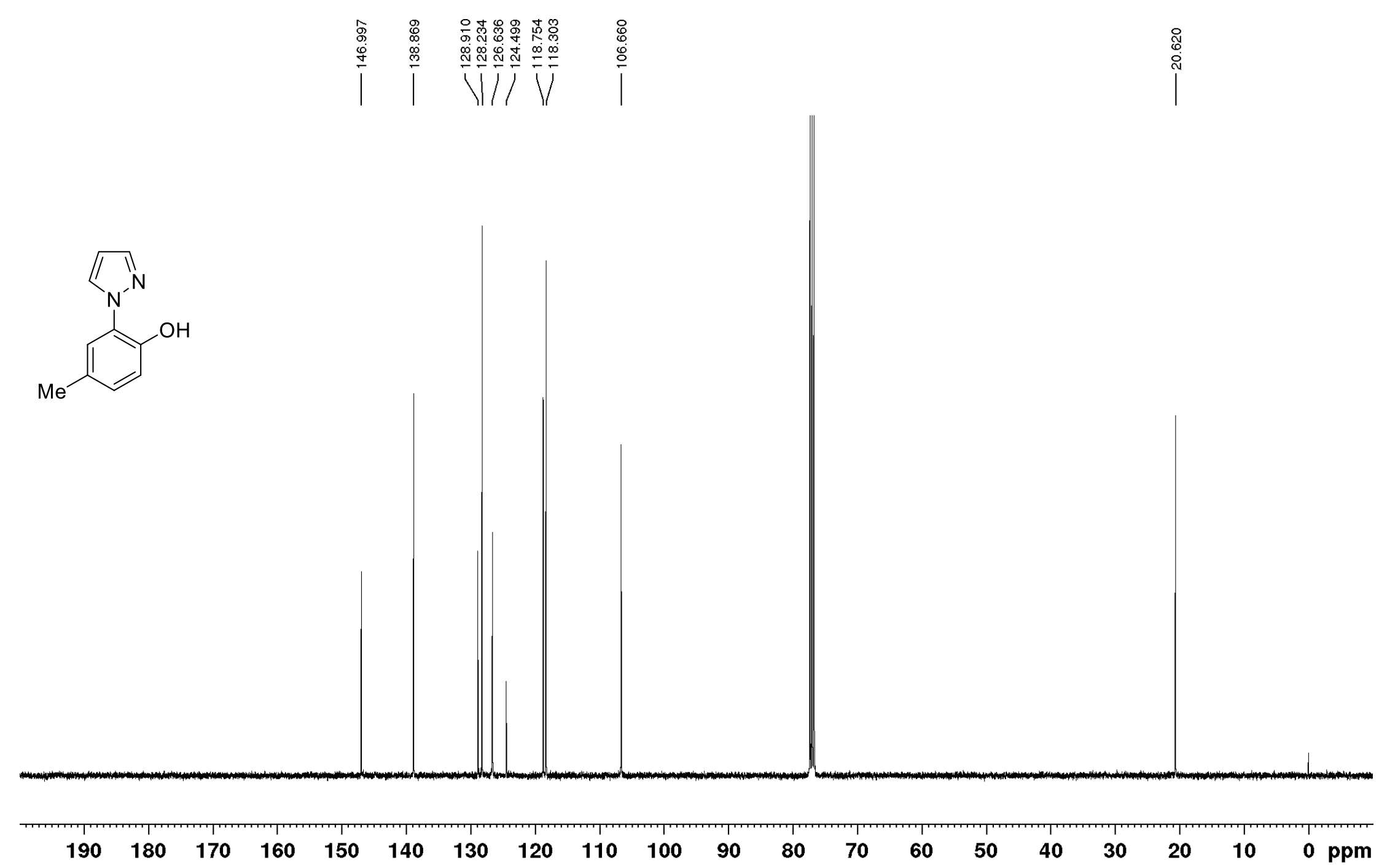


${ }^{1} \mathrm{H}$ NMR $\left(\mathrm{CDCl}_{3}, 400 \mathrm{MHz}\right)$ of 5-Methyl-2-(pyridin-2-yl)phenol (4p)

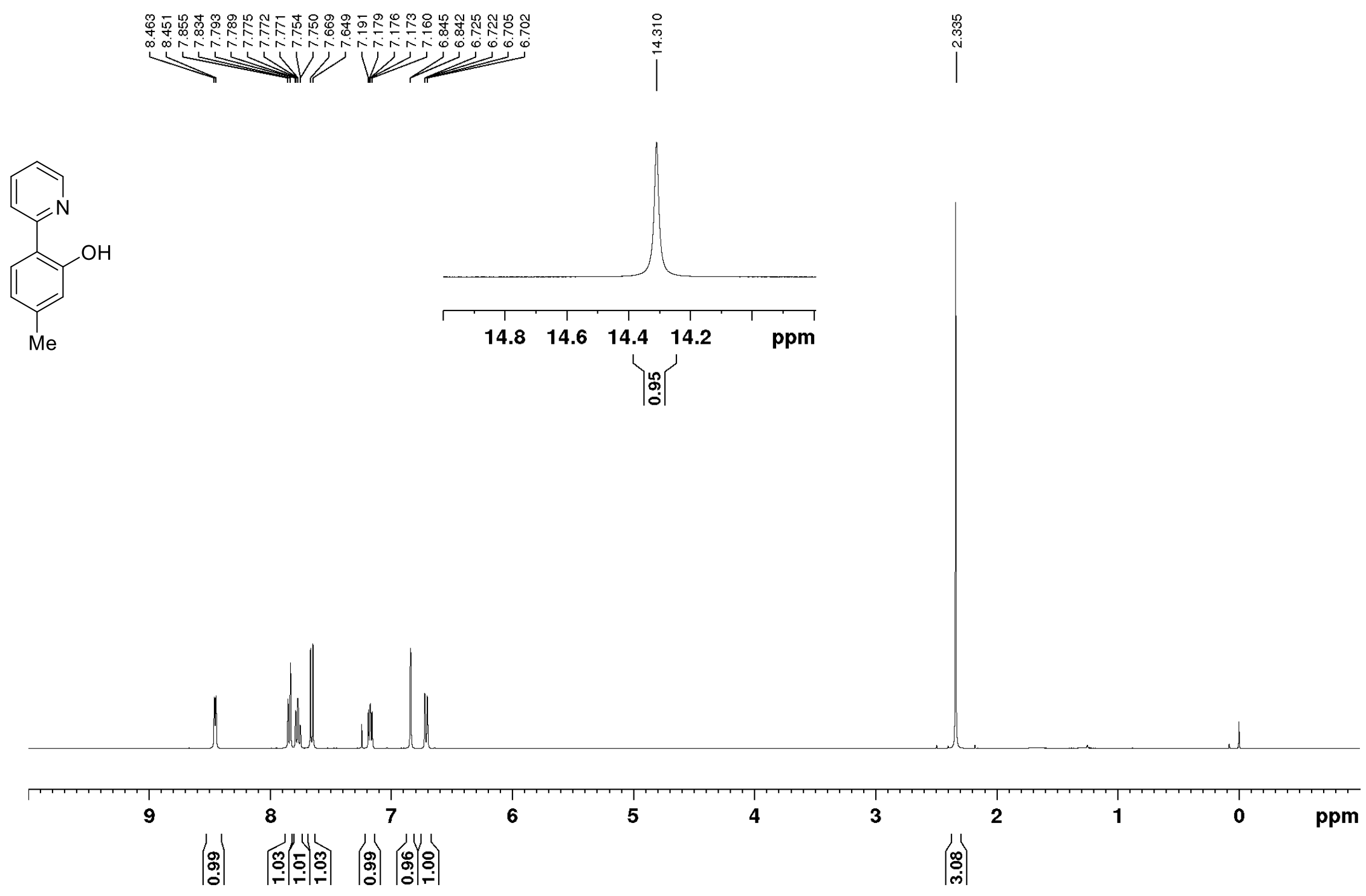


${ }^{13} \mathrm{C}$ NMR $\left(\mathrm{CDCl}_{3}, 100 \mathrm{MHz}\right)$ of 5-Methyl-2-(pyridin-2-yl)phenol (4p)

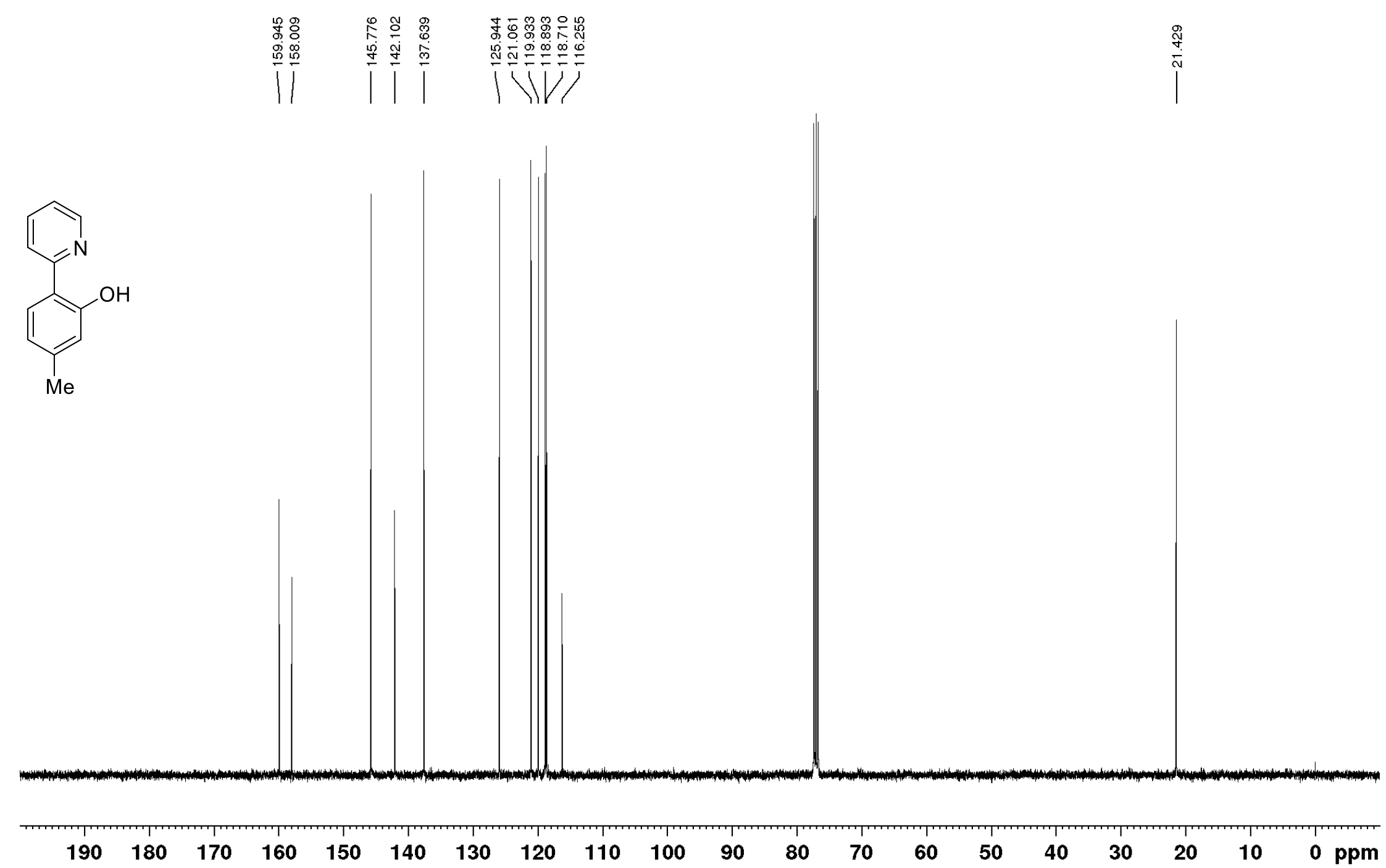


${ }^{1} \mathrm{H}$ NMR $\left(\mathrm{CDCl}_{3}, 400 \mathrm{MHz}\right)$ of 3-Fluoro-2-(pyridin-2-yl)phenol (4q)
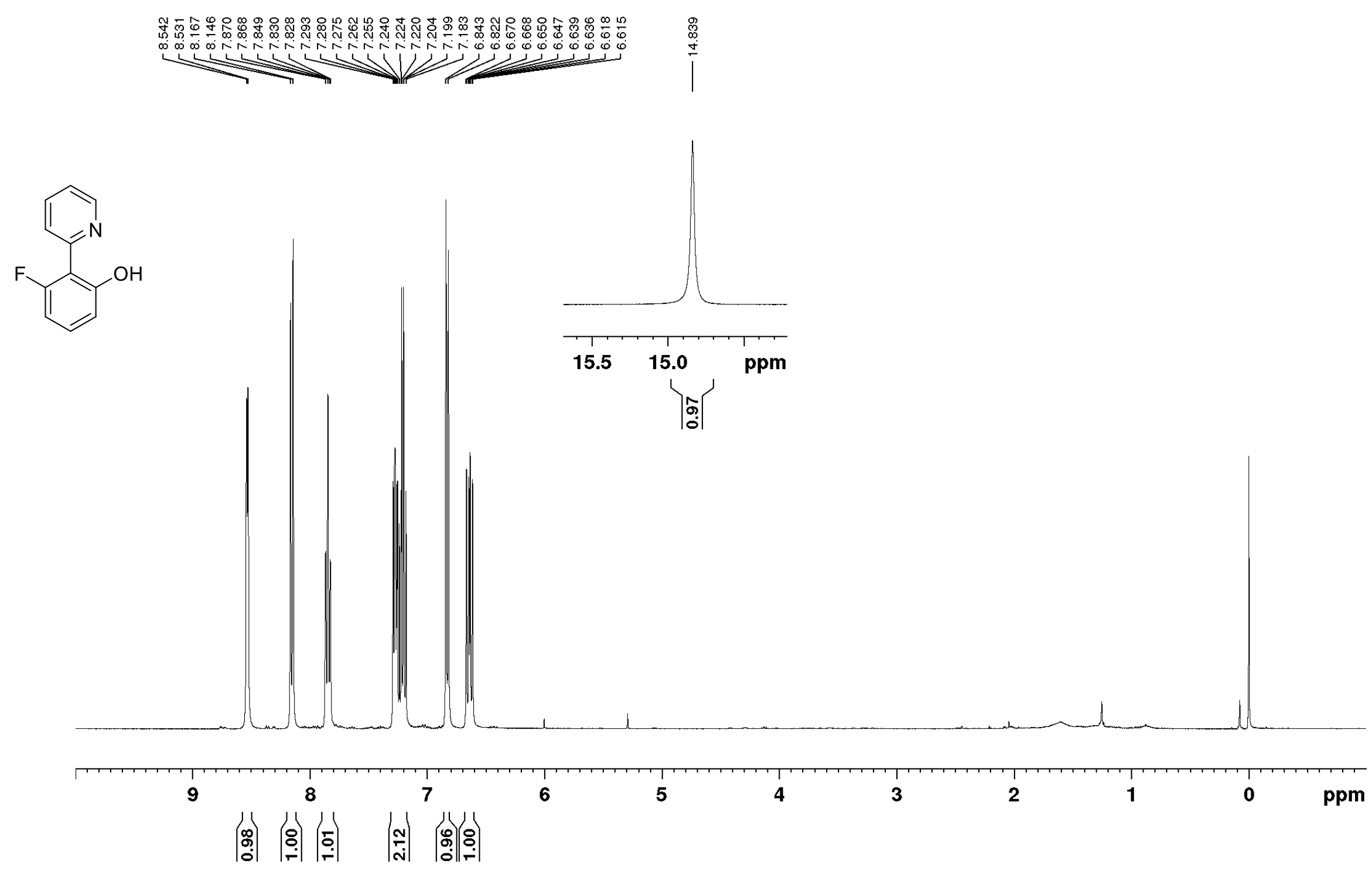


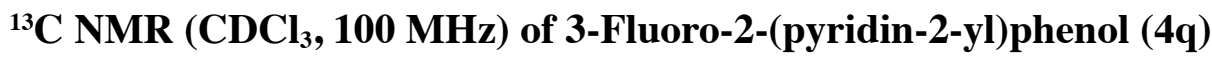

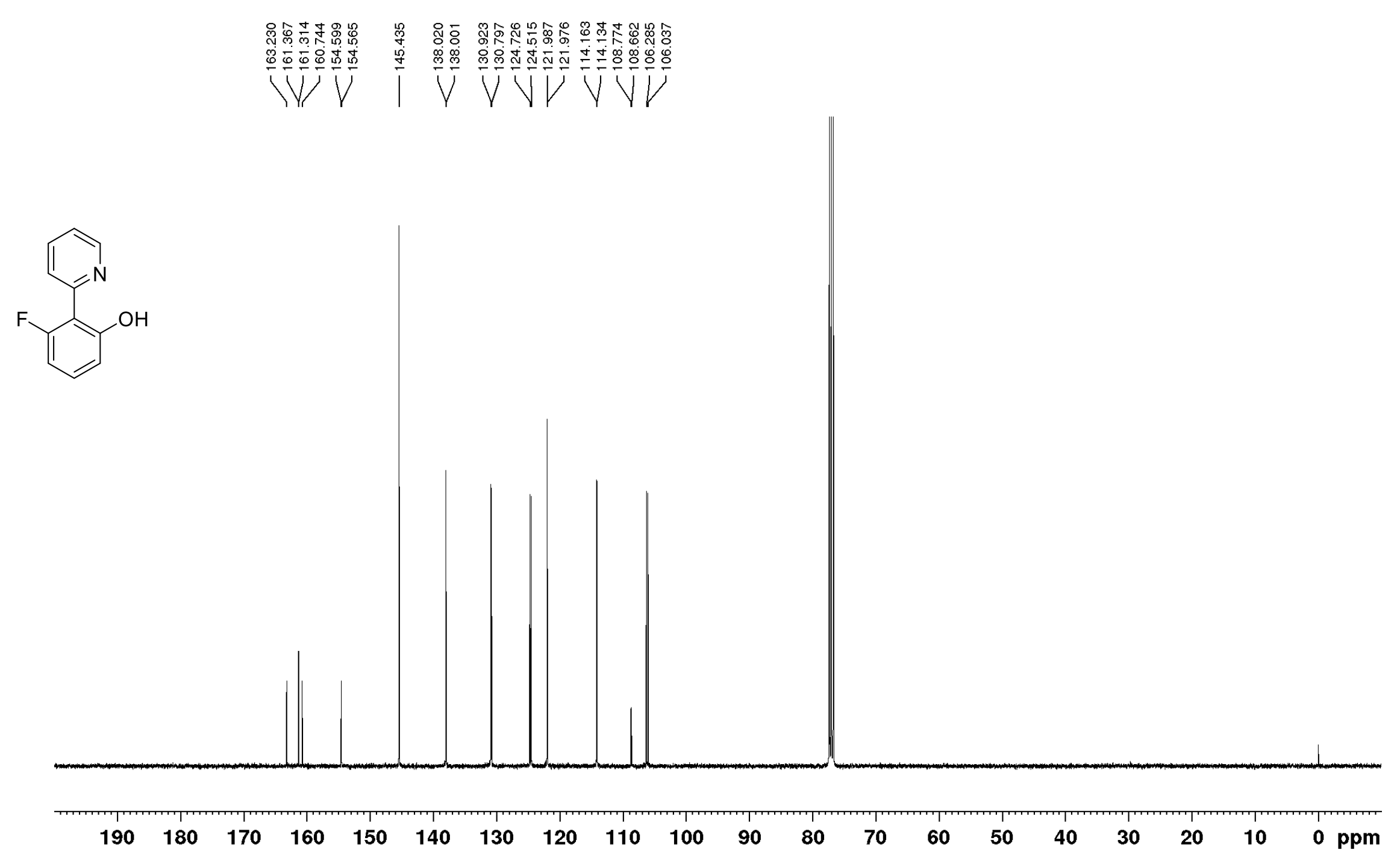




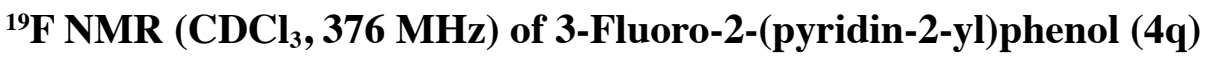

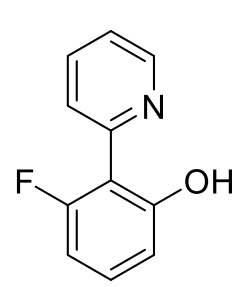

\begin{tabular}{|c|c|c|c|c|c|c|c|c|c|c|}
\hline $\begin{array}{l}1 \\
0\end{array}$ & -20 & -40 & -60 & $\begin{array}{l}1 \\
-80\end{array}$ & -100 & $\begin{array}{c}1 \\
-120\end{array}$ & -140 & -160 & $\begin{array}{c}1 \\
-180\end{array}$ & ppm \\
\hline
\end{tabular}


${ }^{1} \mathrm{H}$ NMR $\left(\mathrm{CDCl}_{3}, 400 \mathrm{MHz}\right)$ of 1-[2-(4,4,5,5-Tetramethyl-1,3,2-dioxaborolan-2-yl)-5-(trifluoromethyl)phenyl]-1H-pyrazole (3r)

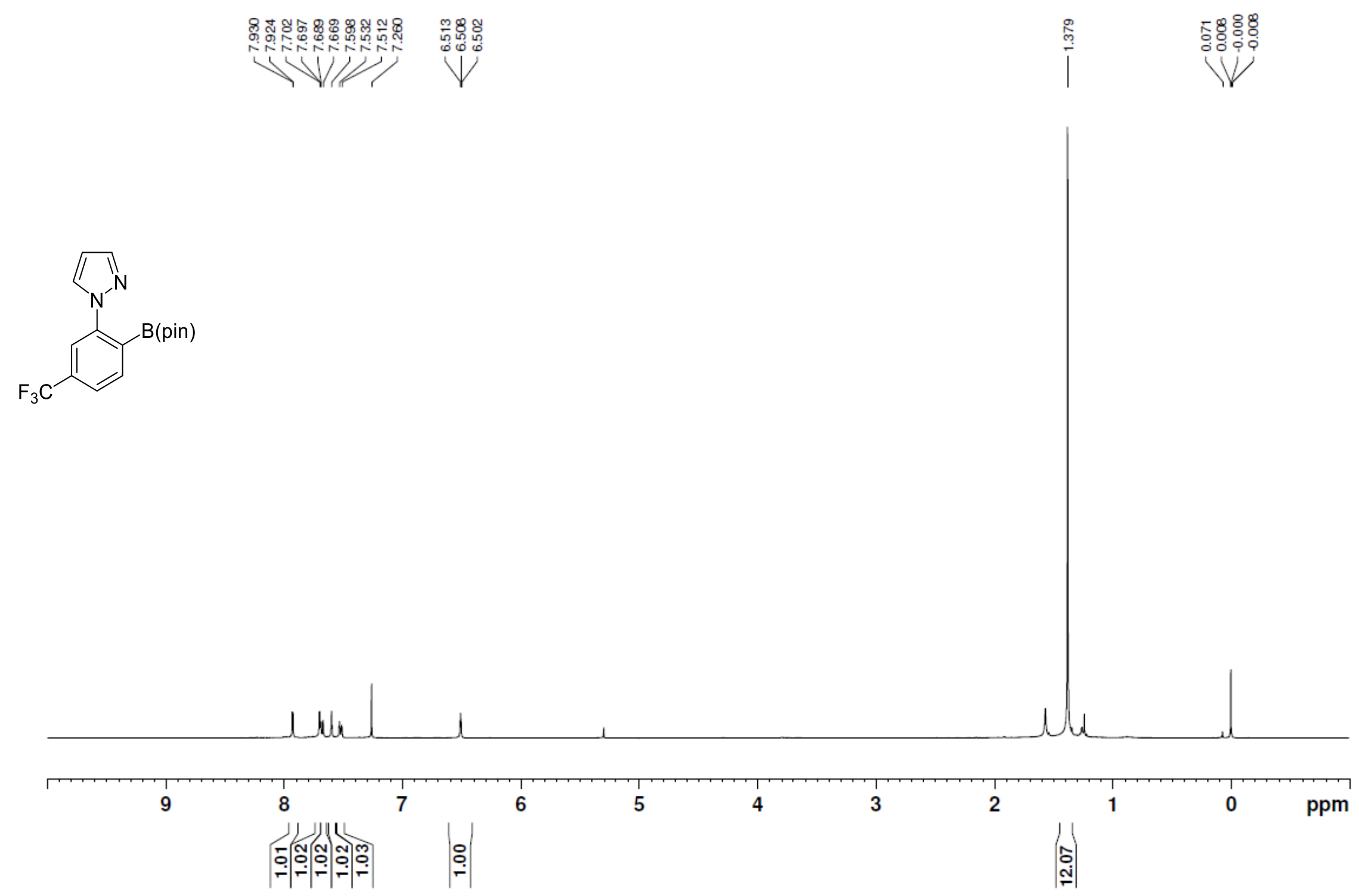


${ }^{13} \mathrm{C} \mathrm{NMR}\left(\mathrm{CDCl}_{3}, 100 \mathrm{MHz}\right)$ of 1-[2-(4,4,5,5-Tetramethyl-1,3,2-dioxaborolan-2-yl)-5-(trifluoromethyl)phenyl]-1H-pyrazole (3r)

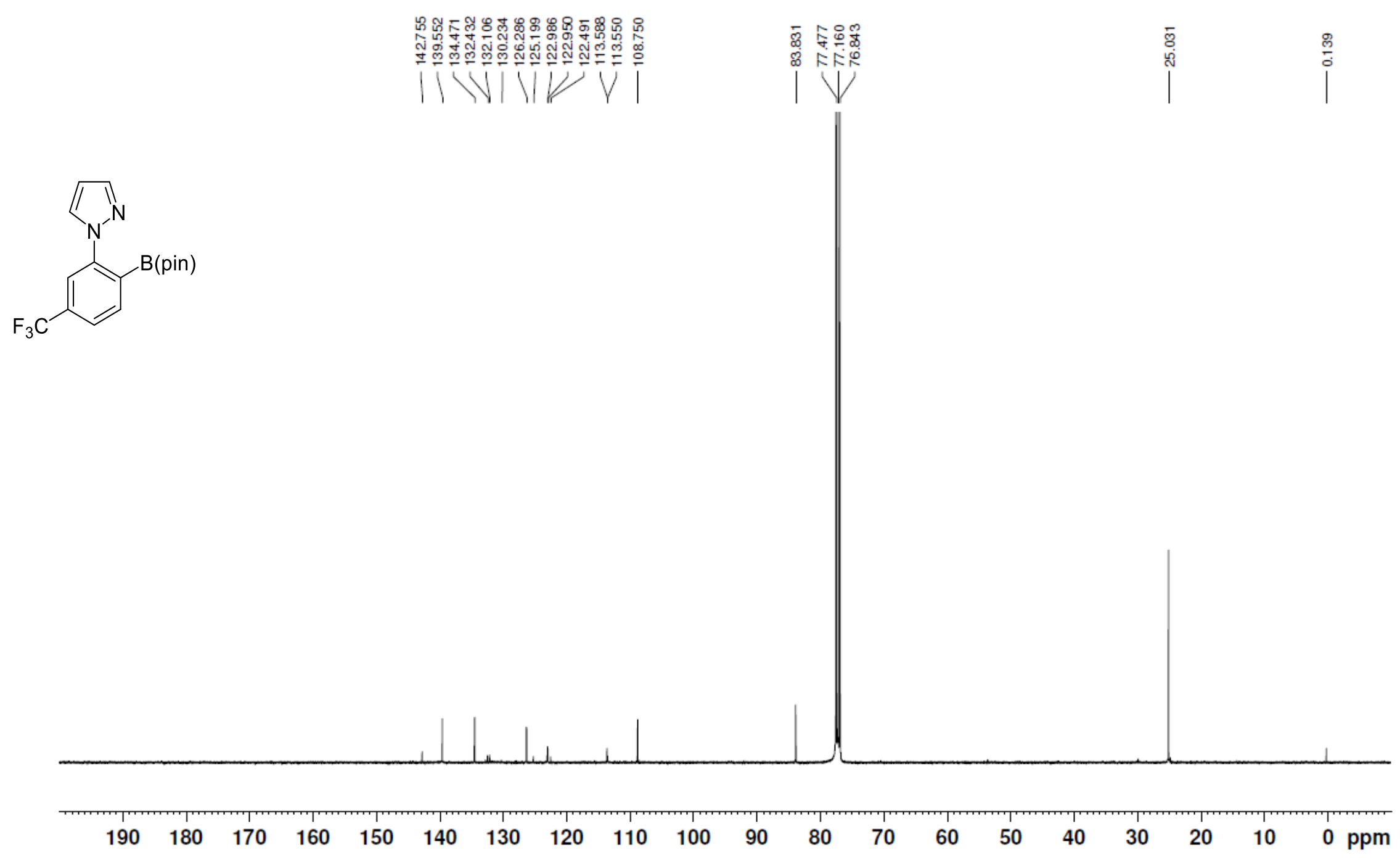


${ }^{19} \mathrm{~F}$ NMR $\left(\mathrm{CDCl}_{3}, 376 \mathrm{MHz}\right)$ of 1-[2-(4,4,5,5-Tetramethyl-1,3,2-dioxaborolan-2-yl)-5-(trifluoromethyl)phenyl]-1H-pyrazole (3r)

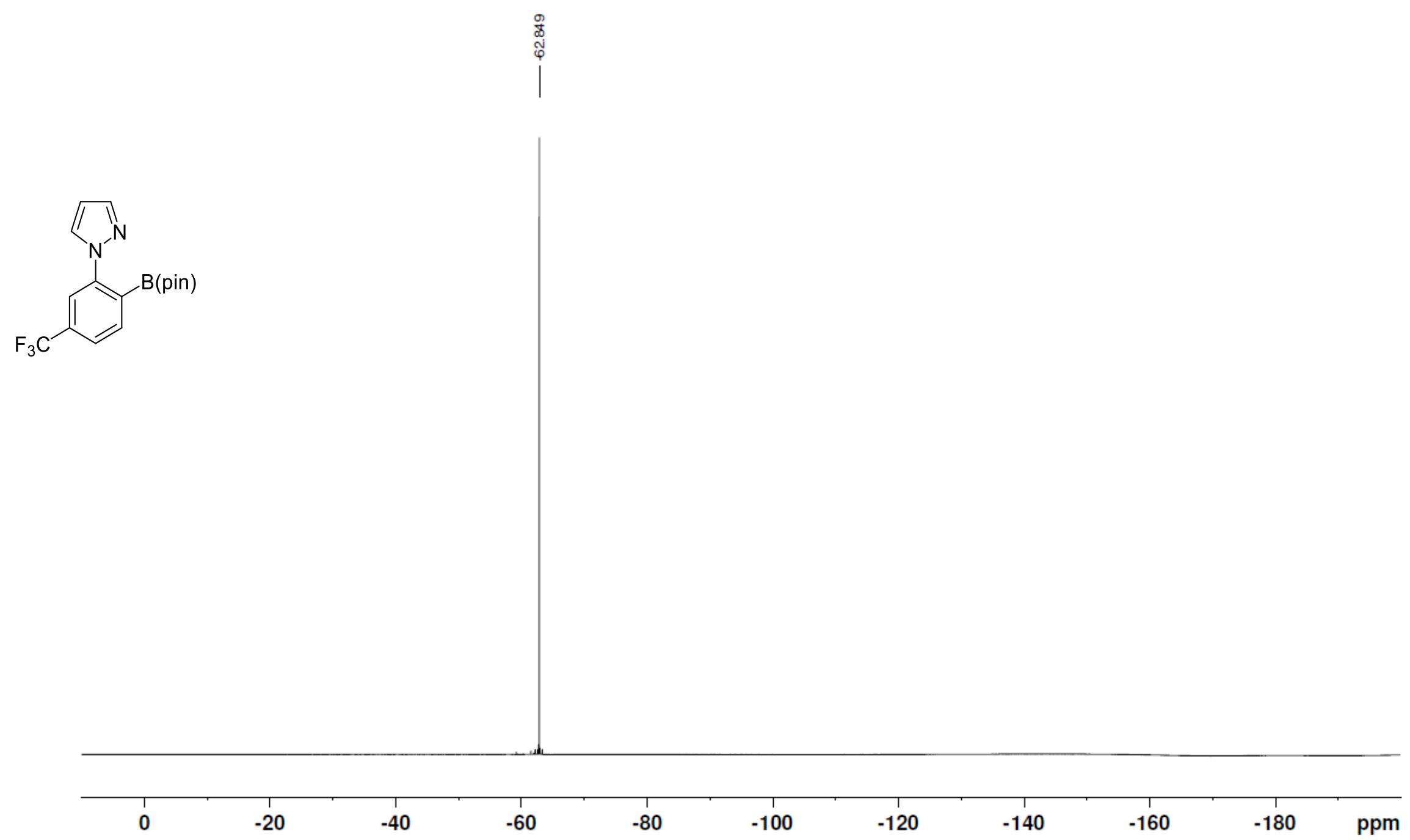


${ }^{1} \mathrm{H}$ NMR $\left(\mathrm{CDCl}_{3}, 400 \mathrm{MHz}\right)$ of 2-(1H-Pyrazol-1-yl)-4-(trifluoromethyl)phenol (4r)

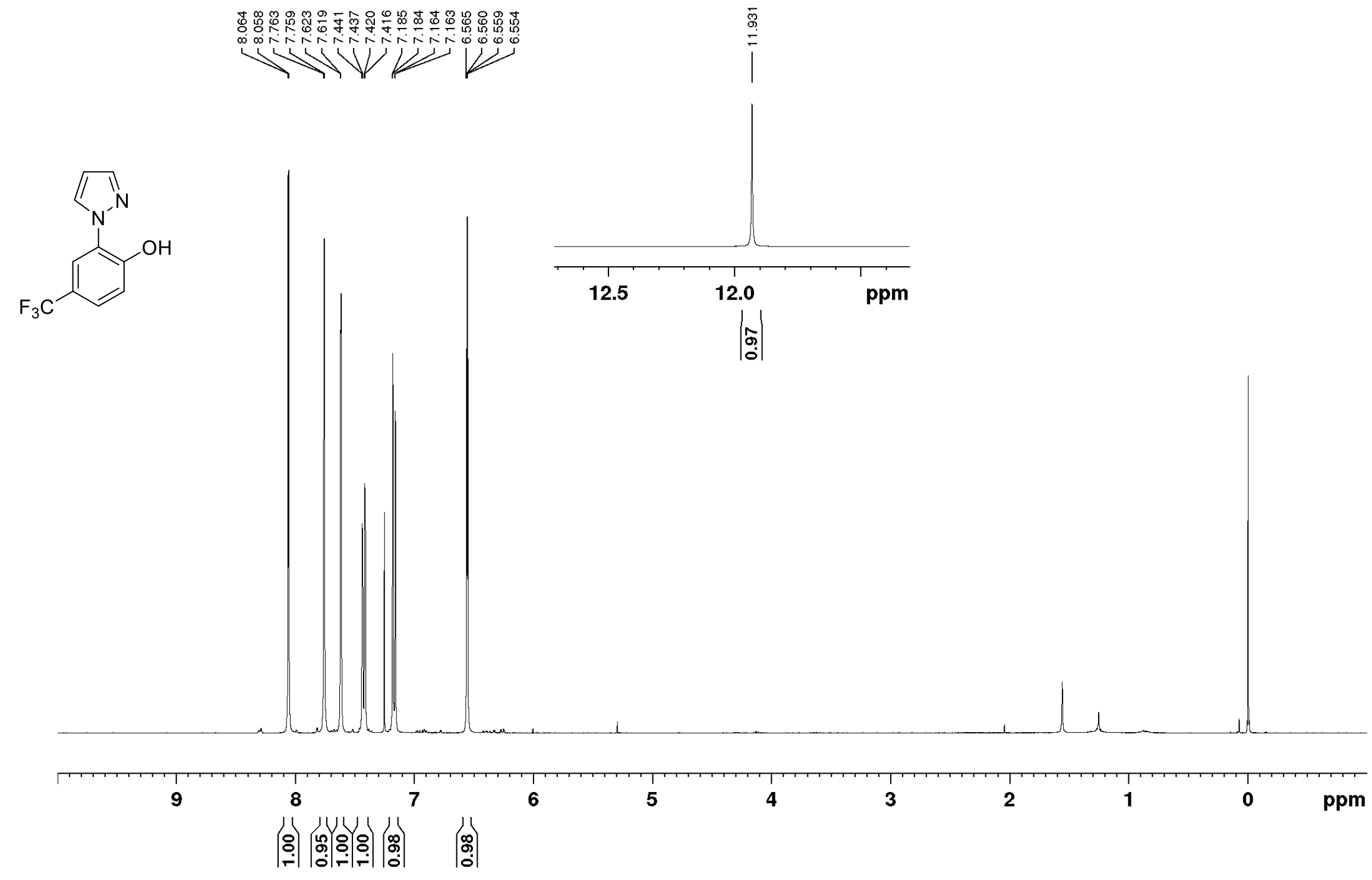


${ }^{13} \mathrm{C}$ NMR (CDCl $\left.3,100 \mathrm{MHz}\right)$ of 2-(1H-Pyrazol-1-yl)-4-(trifluoromethyl)phenol (4r)

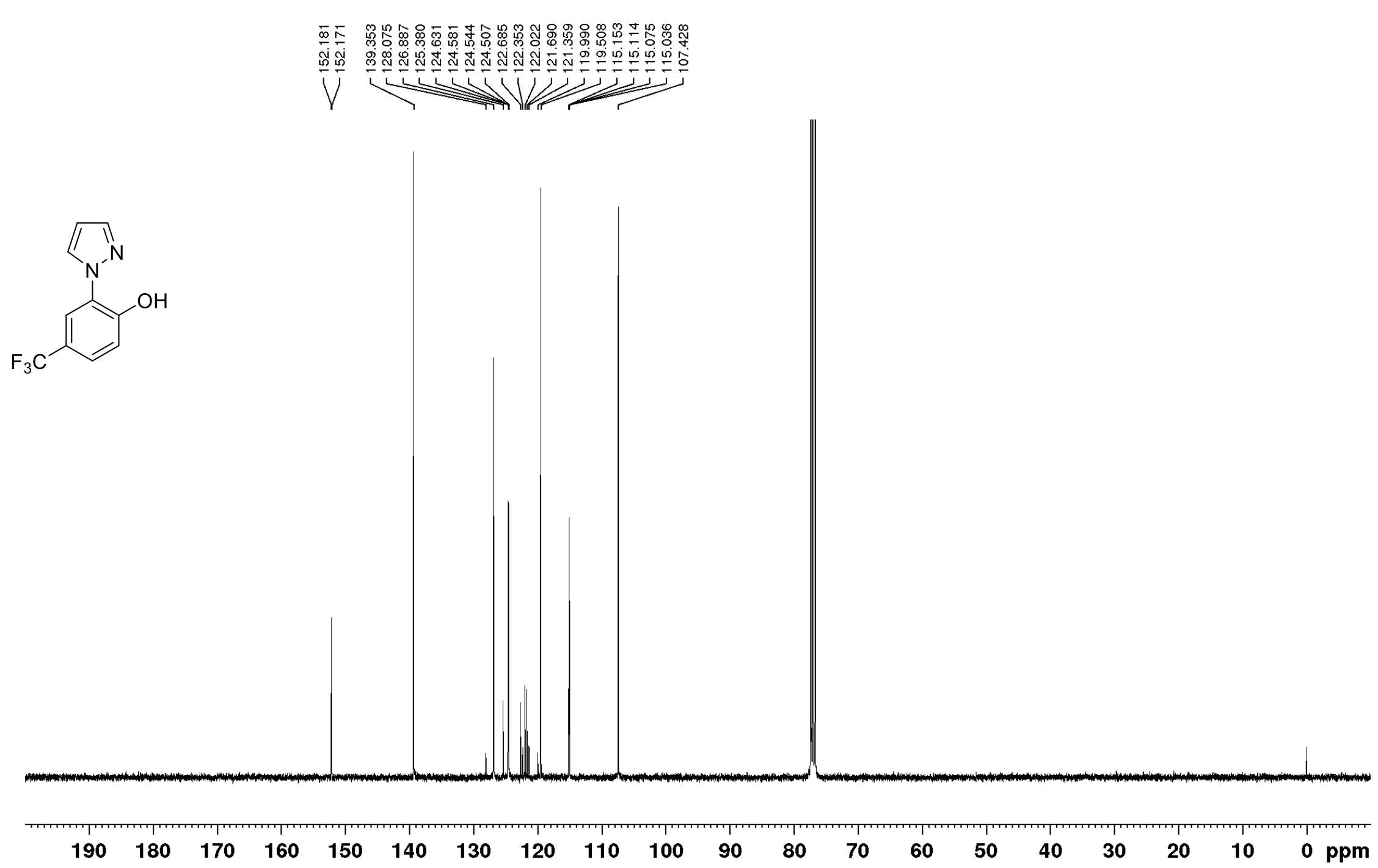


${ }^{19} \mathrm{~F}$ NMR $\left(\mathrm{CDCl}_{3}, 376 \mathrm{MHz}\right)$ of 2-(1H-Pyrazol-1-yl)-4-(trifluoromethyl)phenol (4r)

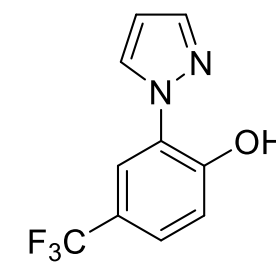

$\mid$

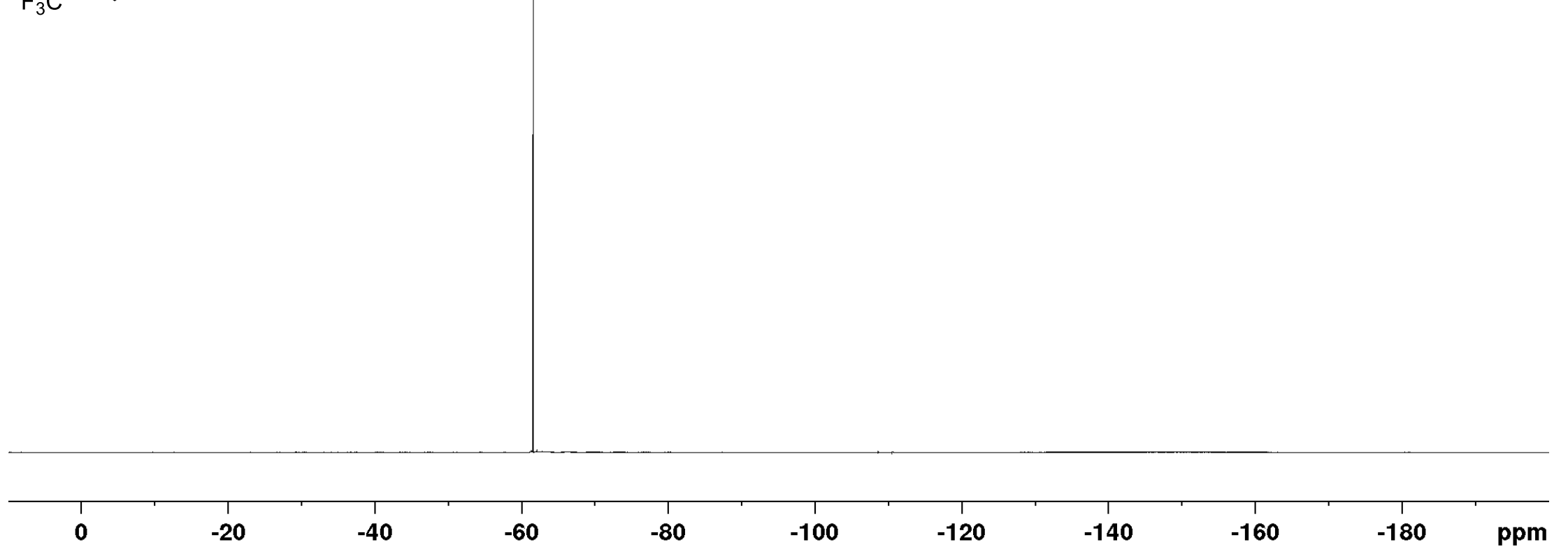


${ }^{1} \mathrm{H}$ NMR $\left(\mathrm{CDCl}_{3}, 400 \mathrm{MHz}\right)$ of 1-[5-Chloro-2-(4,4,5,5-tetramethyl-1,3,2-dioxaborolan-2-yl)phenyl]-1H-pyrazole (3s)
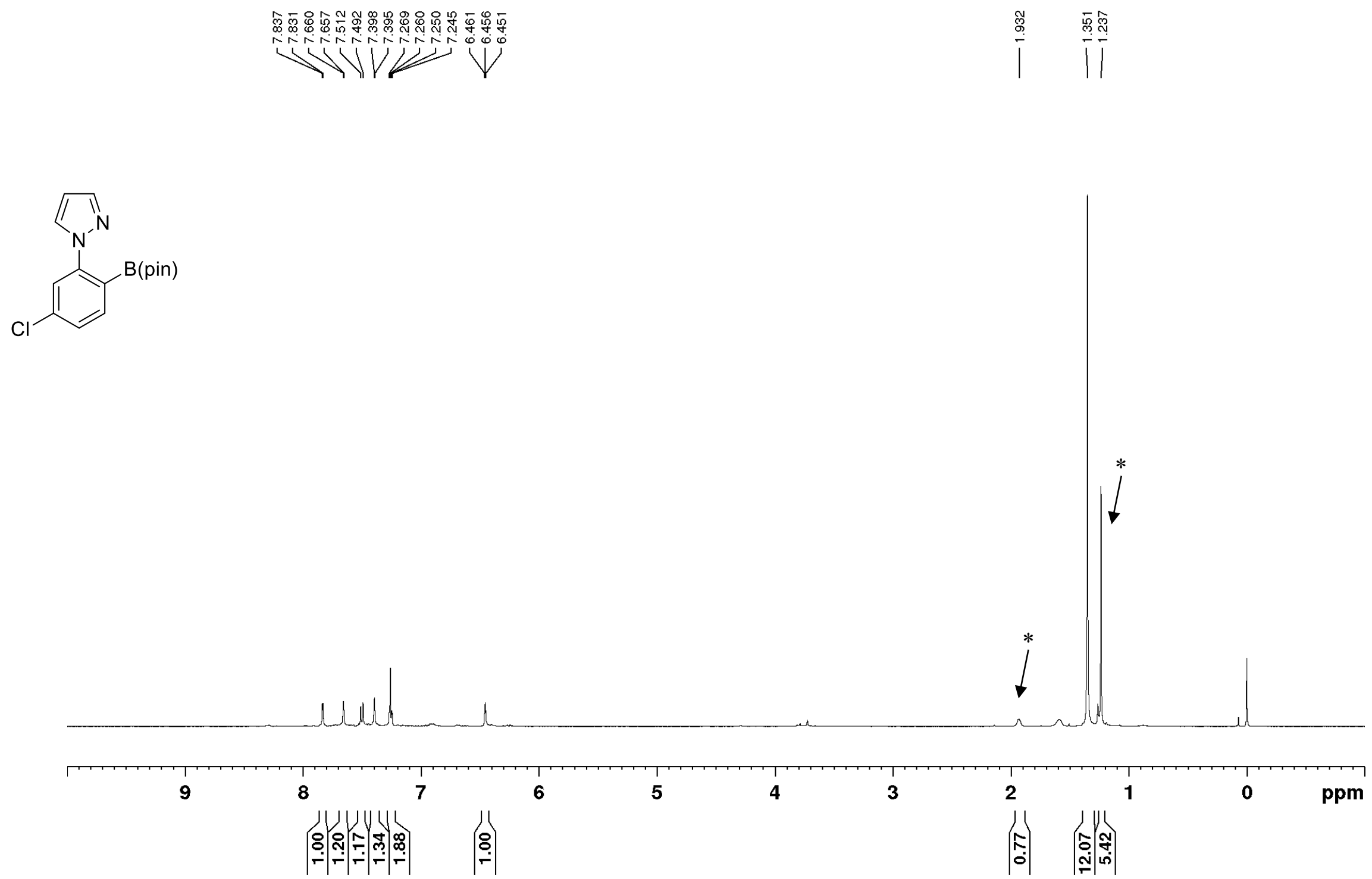
${ }^{13} \mathrm{C}$ NMR $\left(\mathrm{CDCl}_{3}, 100 \mathrm{MHz}\right)$ of 1-[5-Chloro-2-(4,4,5,5-tetramethyl-1,3,2-dioxaborolan-2-yl)phenyl]-1H-pyrazole (3s)

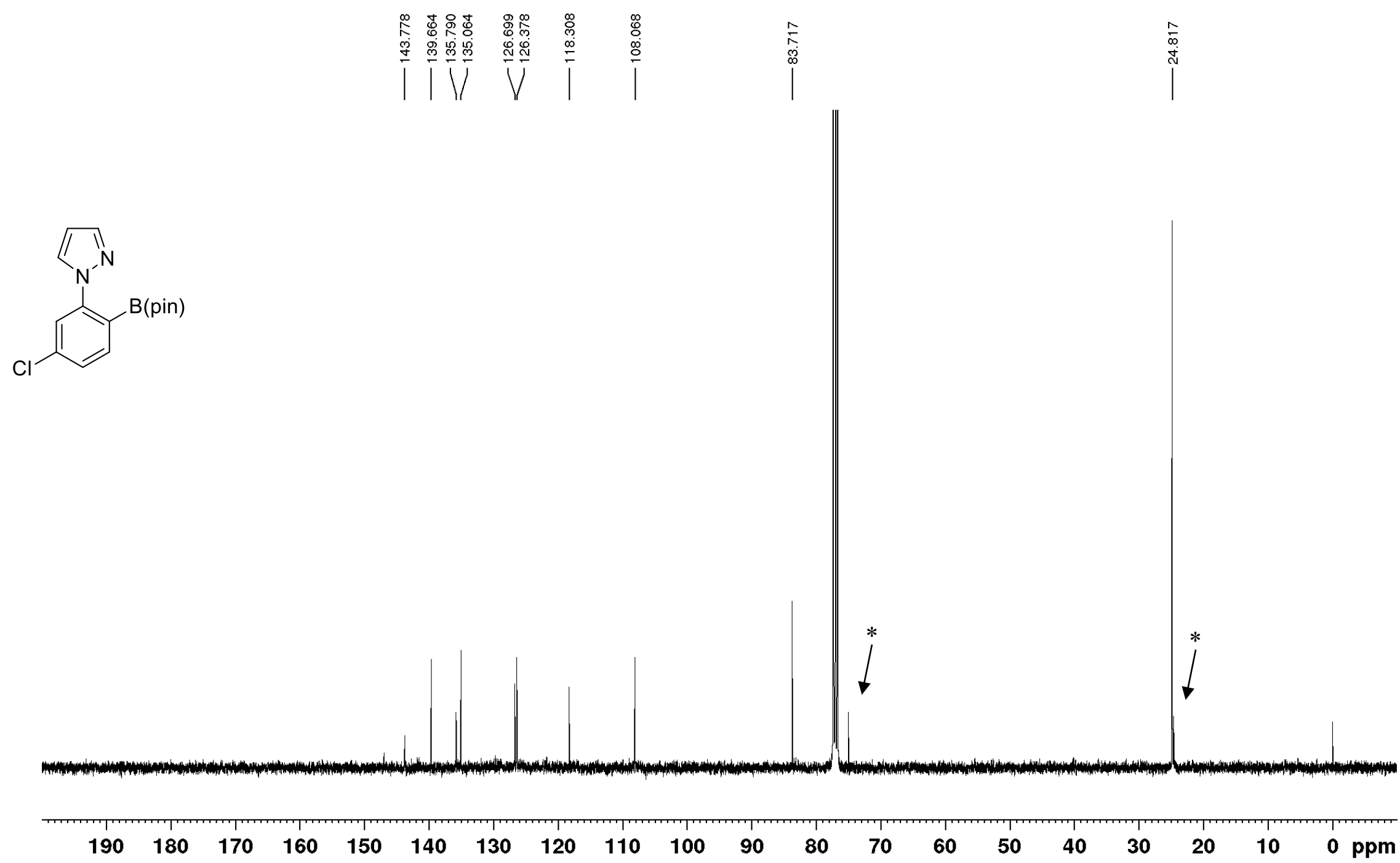


${ }^{1} \mathrm{H}$ NMR $\left(\mathrm{CDCl}_{3}, 400 \mathrm{MHz}\right)$ of 4-Methoxy-2-(pyridin-2-yl)phenol (4t)

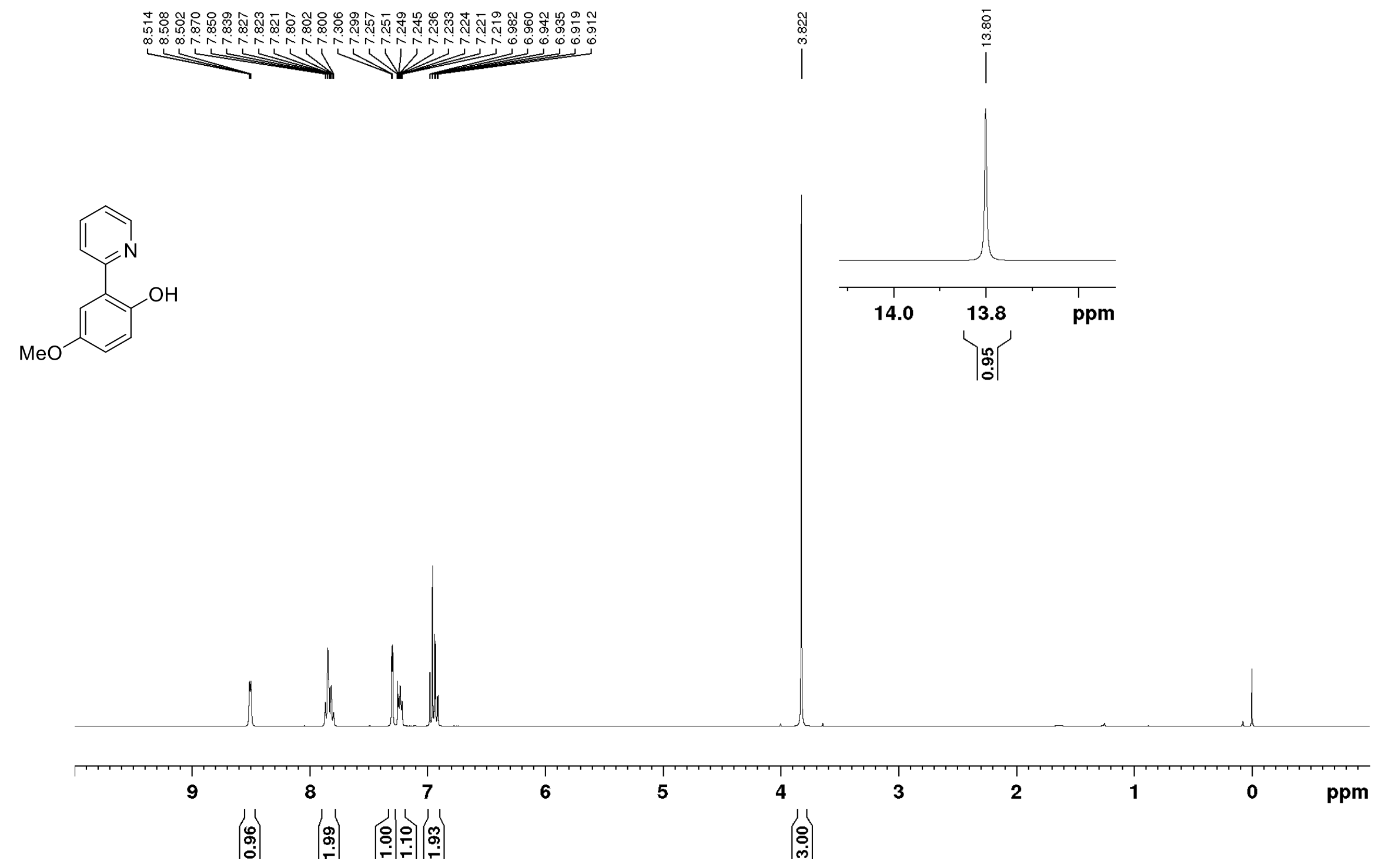


${ }^{13} \mathrm{C} \mathrm{NMR}\left(\mathrm{CDCl}_{3}, 100 \mathrm{MHz}\right)$ of 4-Methoxy-2-(pyridin-2-yl)phenol (4t)
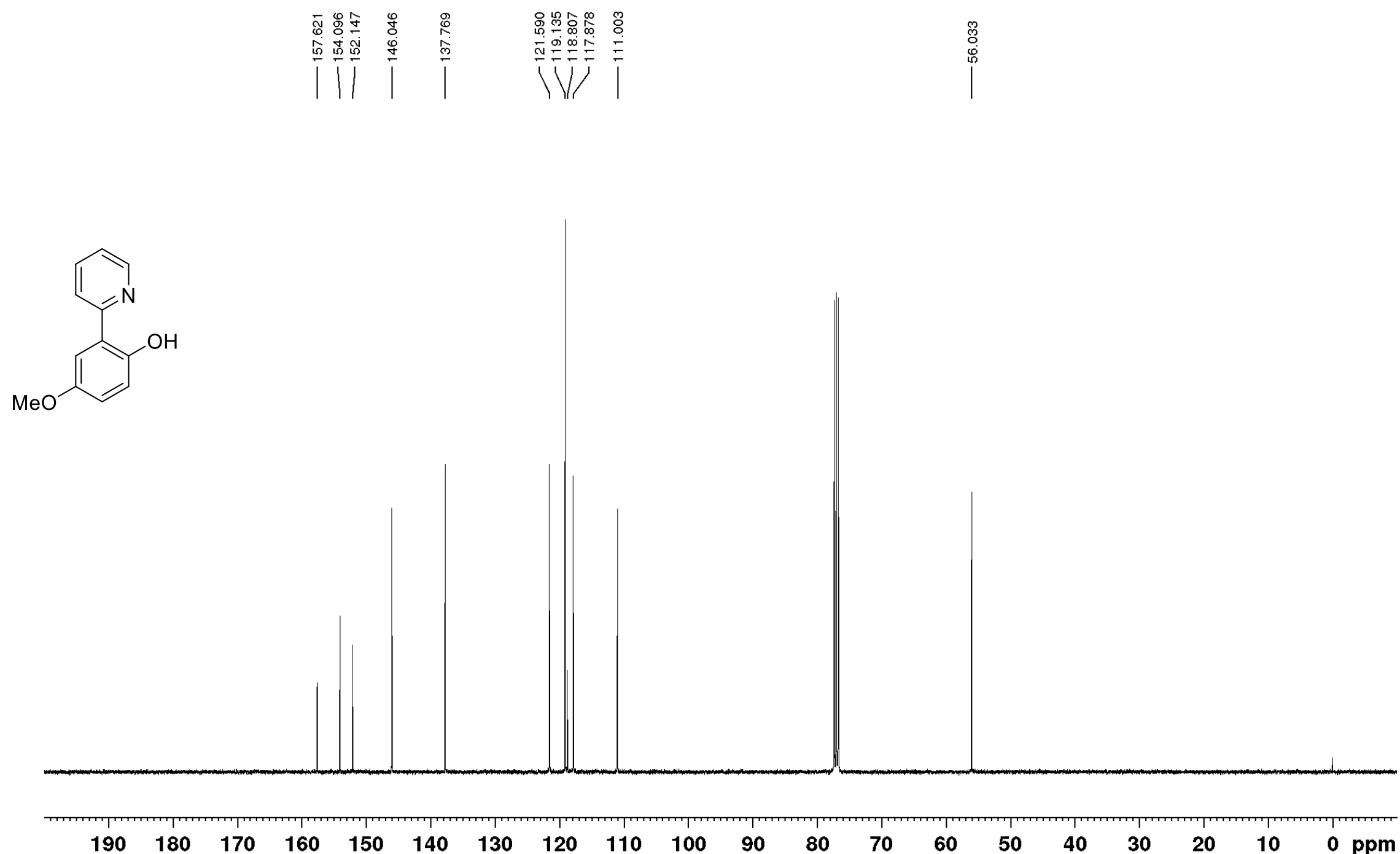
${ }^{1} \mathrm{H}$ NMR $\left(\mathrm{CDCl}_{3}, 400 \mathrm{MHz}\right)$ of 2-(Pyridin-2-yl)-5-(trifluoromethyl)phenol (4u)
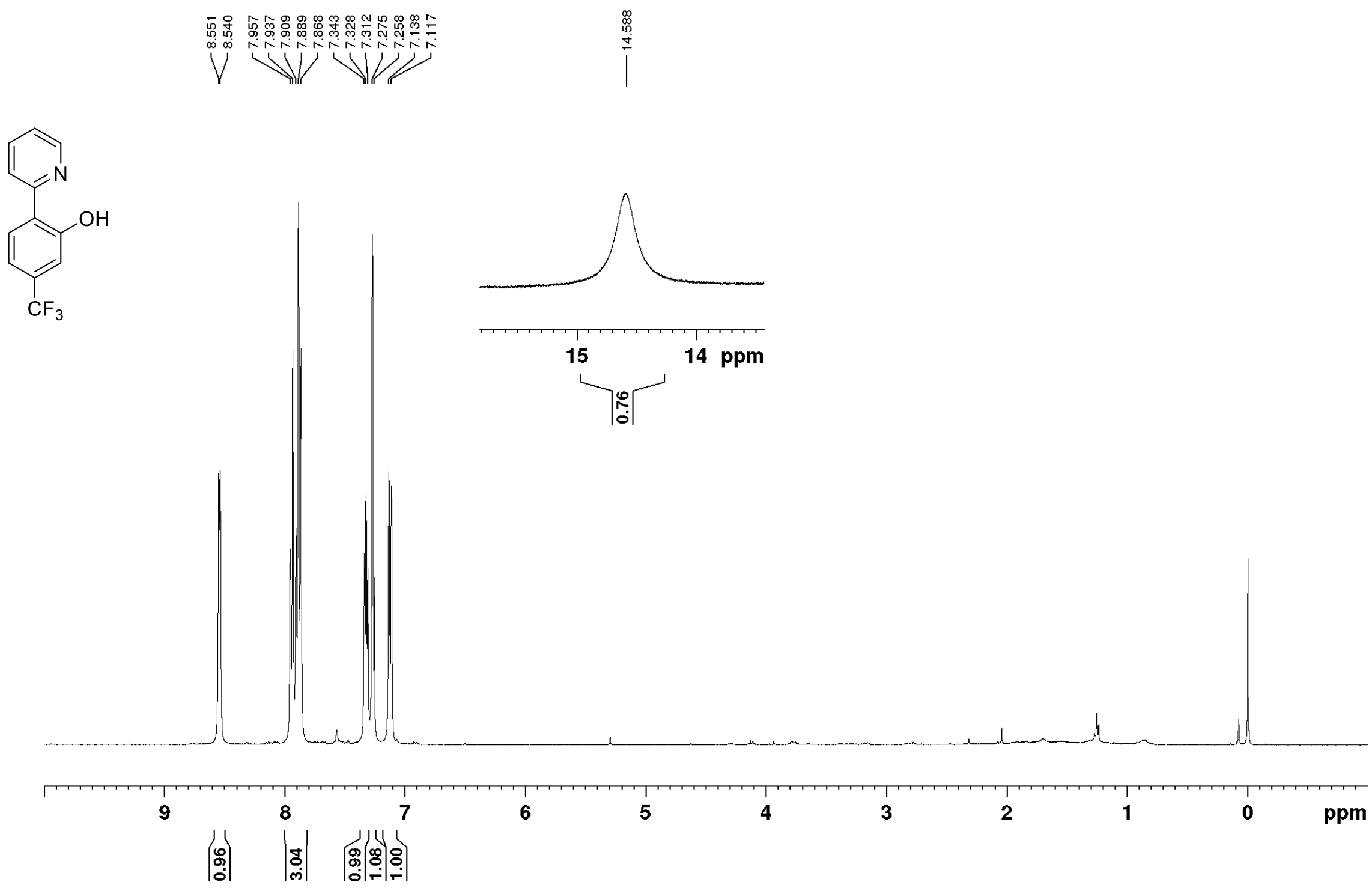
${ }^{13} \mathrm{C}$ NMR $\left(\mathrm{CDCl}_{3}, 100 \mathrm{MHz}\right)$ of 2-(Pyridin-2-yl)-5-(trifluoromethyl)phenol (4u)
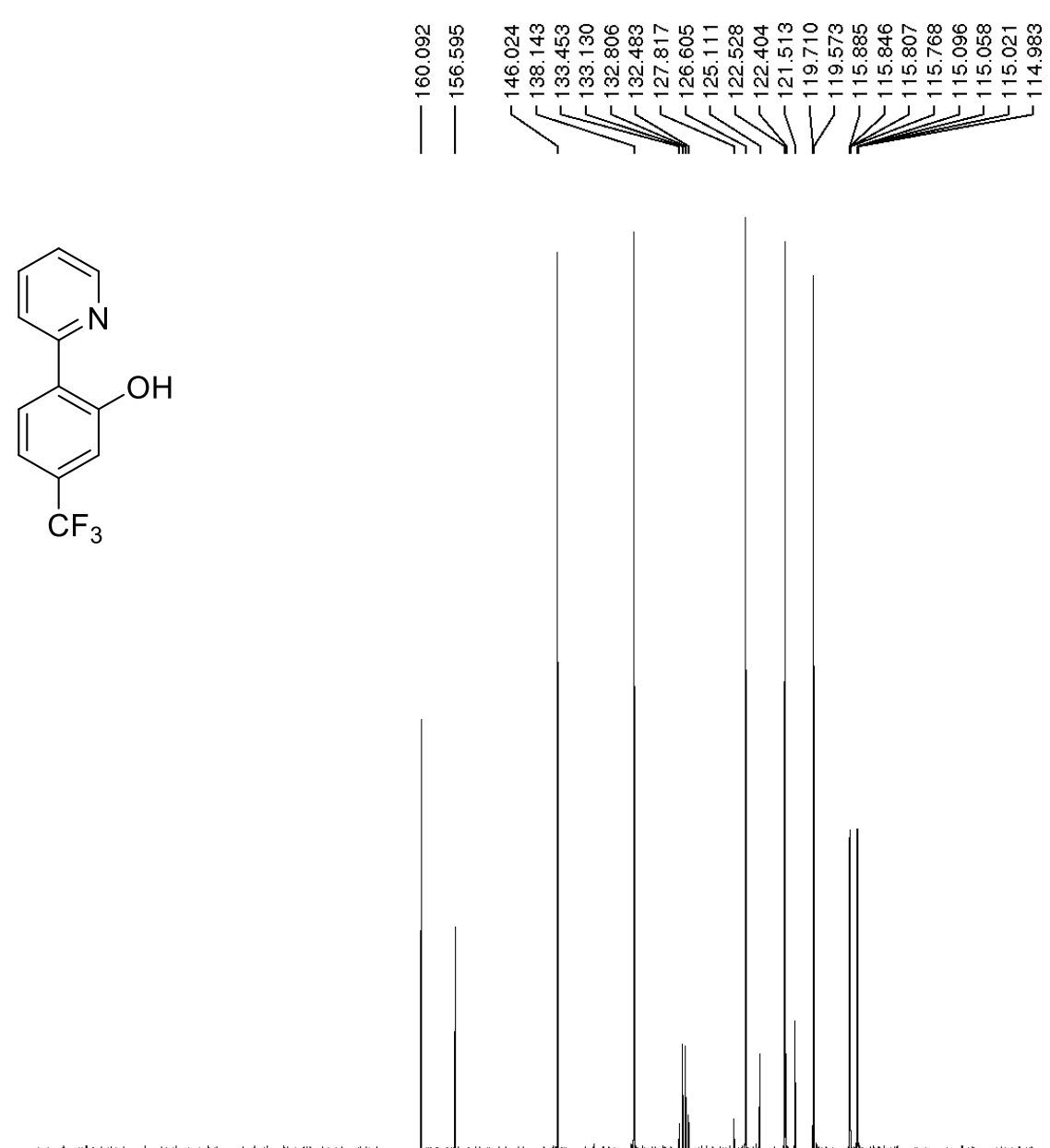

$\begin{array}{llllllllll}190 & 180 & 170 & 160 & 150 & 140 & 130 & 120 & 110 & 100\end{array}$

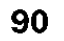

80

70

60

50

40

30

20

10

0 ppm 
${ }^{19} \mathrm{~F}$ NMR $\left(\mathrm{CDCl}_{3}, 376 \mathrm{MHz}\right)$ of 2-(Pyridin-2-yl)-5-(trifluoromethyl)phenol (4u)

|
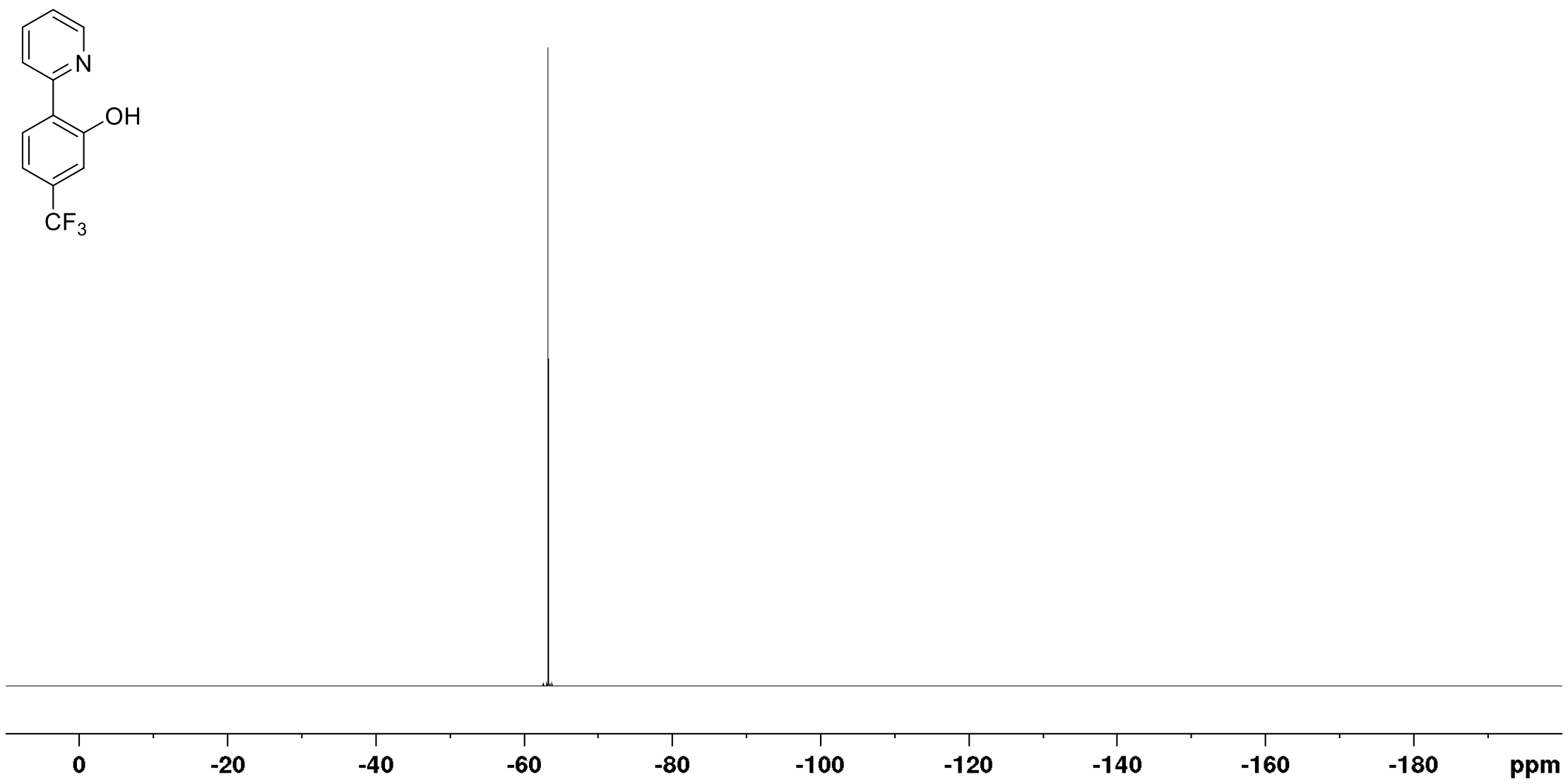
${ }^{1} \mathrm{H}$ NMR $\left(\mathrm{CDCl}_{3}, 400 \mathrm{MHz}\right)$ of 1-\{3-(4,4,5,5-Tetramethyl-1,3,2-dioxaborolan-2-yl)-[1,1'-biphenyl]-4-yl $\}$-1H-pyrazole (3v)

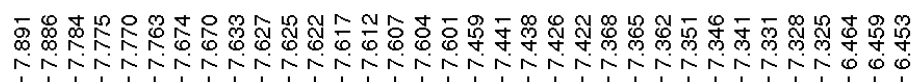

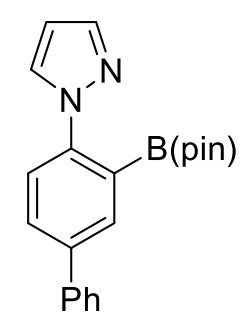

ind If

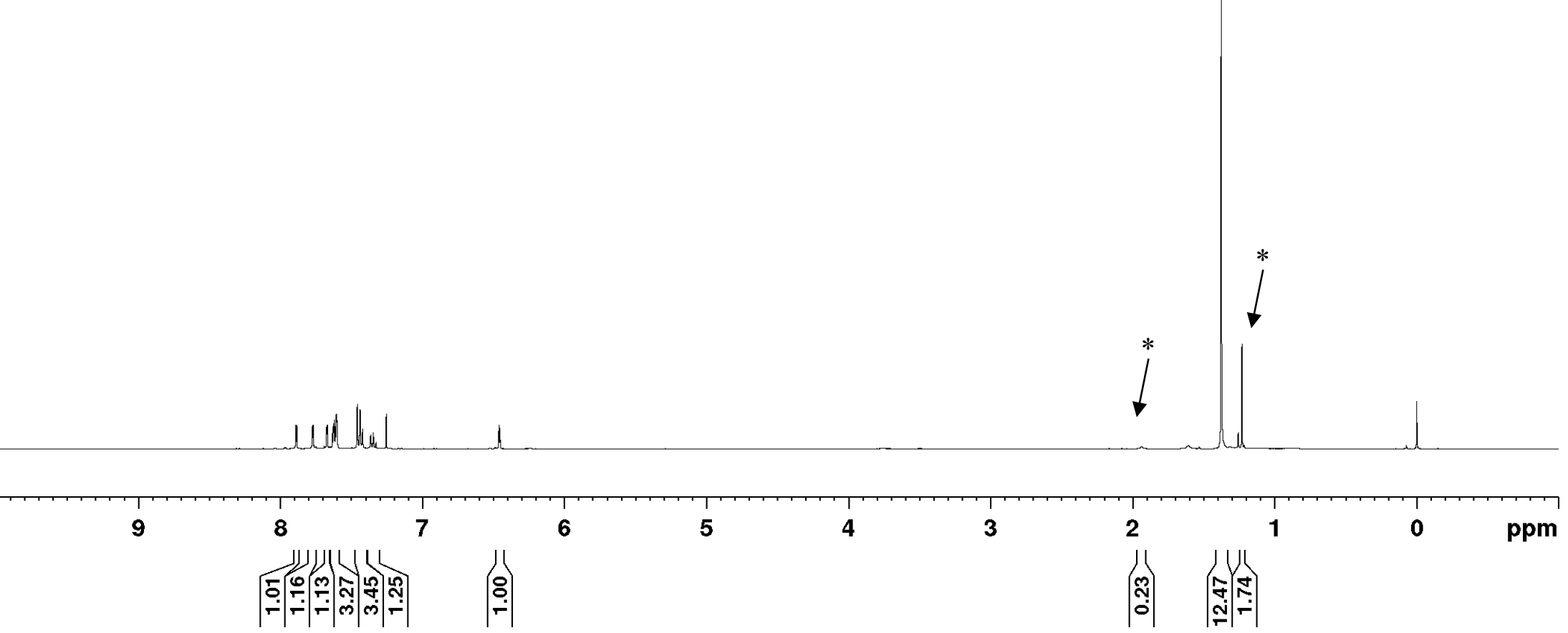


${ }^{13} \mathrm{C}$ NMR $\left(\mathrm{CDCl}_{3}, 100 \mathrm{MHz}\right)$ of 1-\{3-(4,4,5,5-Tetramethyl-1,3,2-dioxaborolan-2-yl)-[1,1'-biphenyl]-4-yl\}-1H-pyrazole (3v)

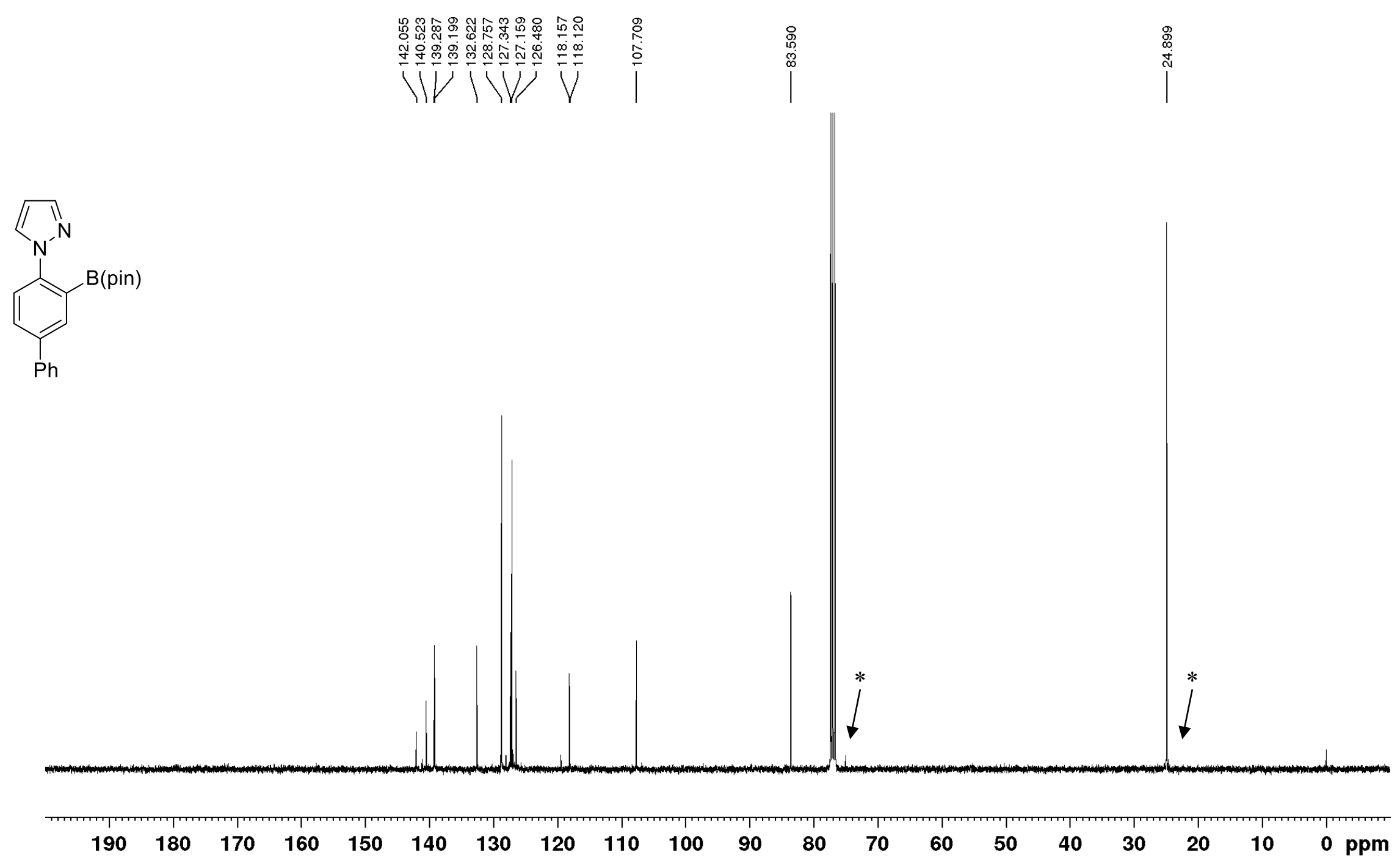


${ }^{1} \mathrm{H}$ NMR $\left(\mathrm{CDCl}_{3}, 400 \mathrm{MHz}\right)$ of 5-Bromo-2-(pyridin-2-yl)phenol (4w)
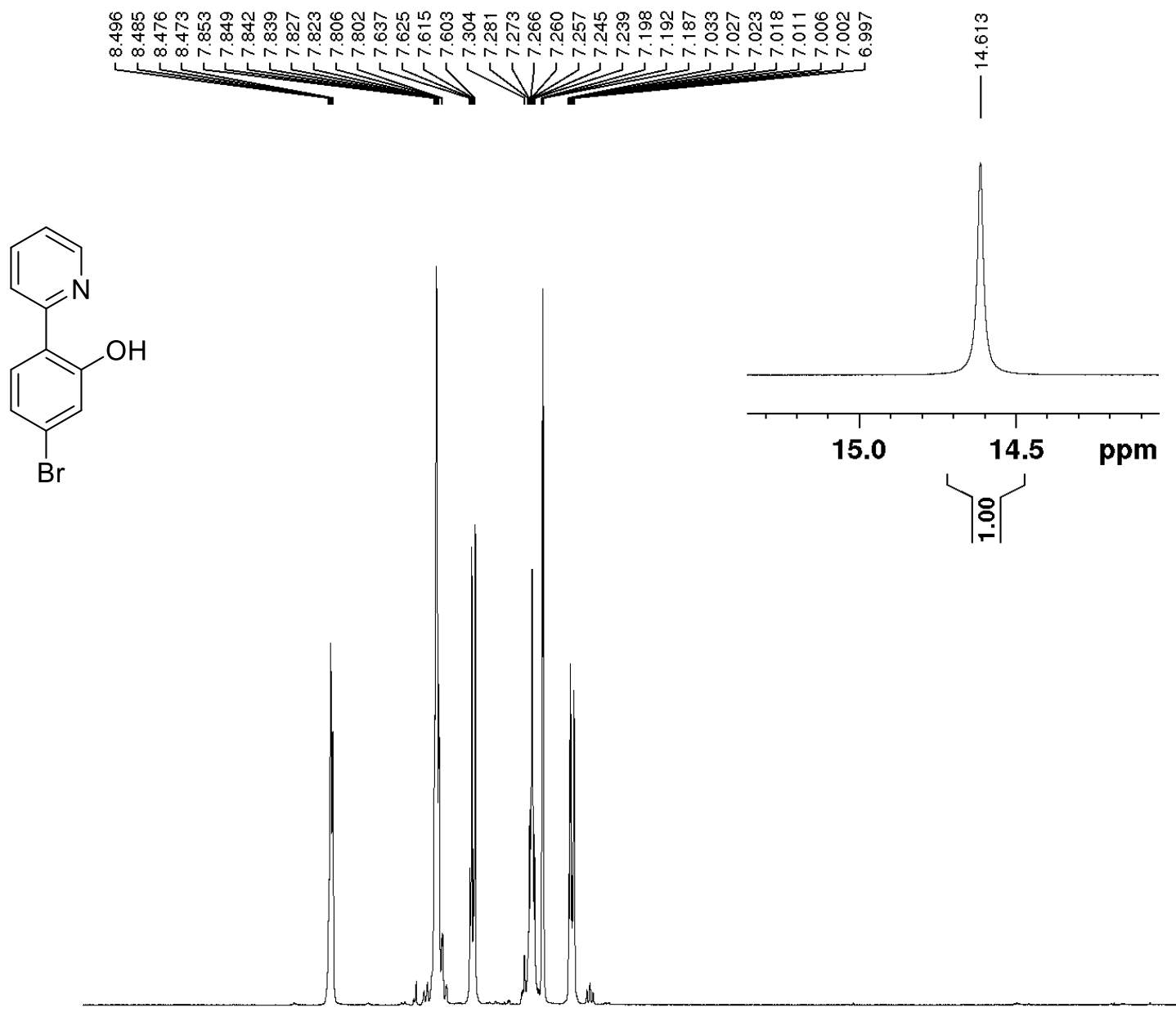

9

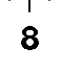

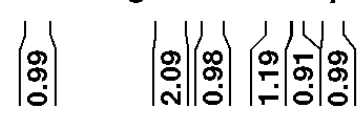


${ }^{13} \mathrm{C}$ NMR $\left(\mathrm{CDCl}_{3}, 100 \mathrm{MHz}\right)$ of 5-Bromo-2-(pyridin-2-yl)phenol (4w)
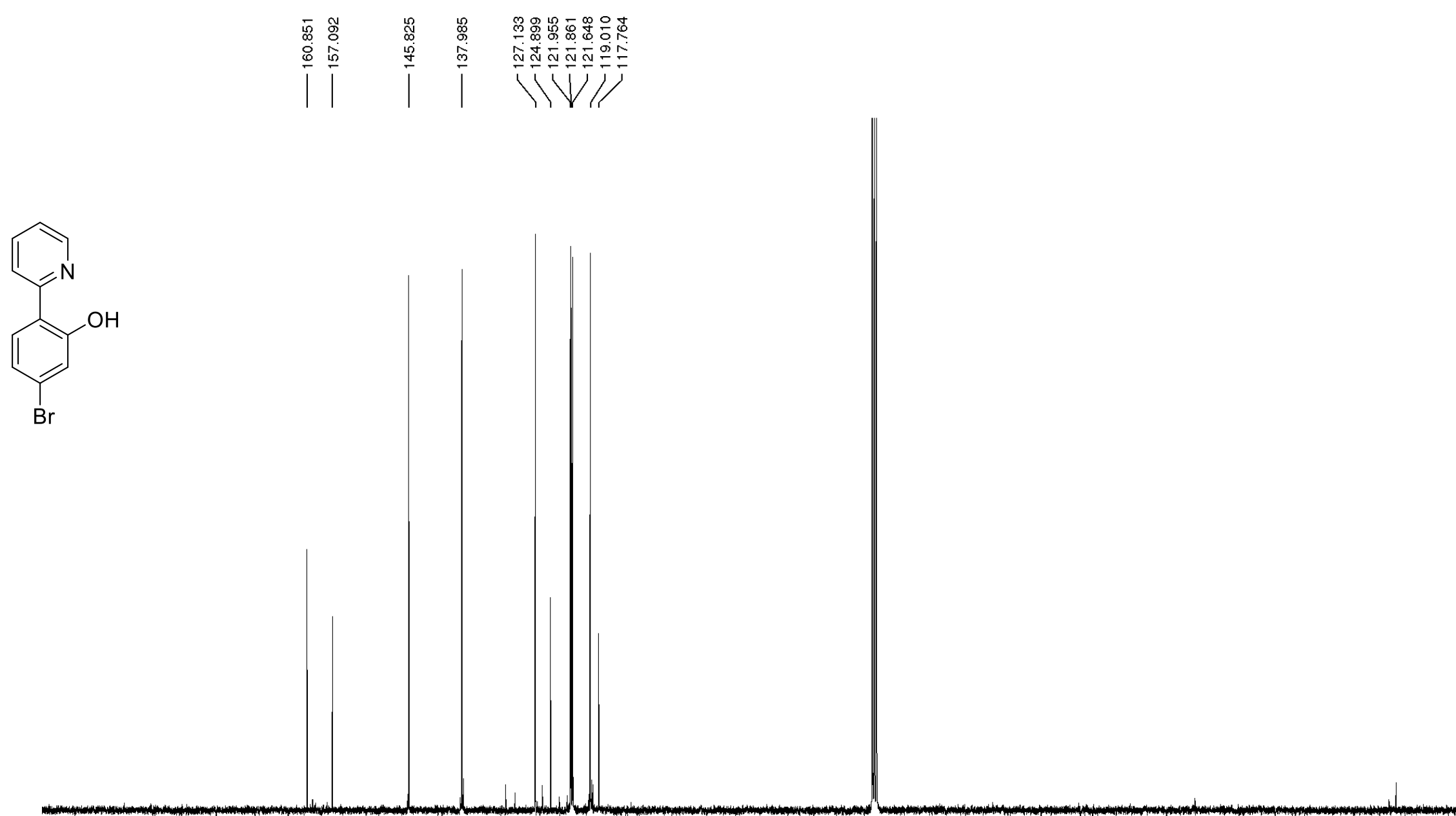

$\begin{array}{llllllllll}190 & 180 & 170 & 160 & 150 & 140 & 130 & 120 & 110 & 100\end{array}$

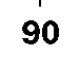

80

$70 \quad 60$ 
${ }^{1} \mathrm{H}$ NMR $\left(\mathrm{CDCl}_{3}, 400 \mathrm{MHz}\right)$ of 1-[4-Methoxy-2-(4,4,5,5-tetramethyl-1,3,2-dioxaborolan-2-yl)phenyl]-1H-pyrazole (3x)
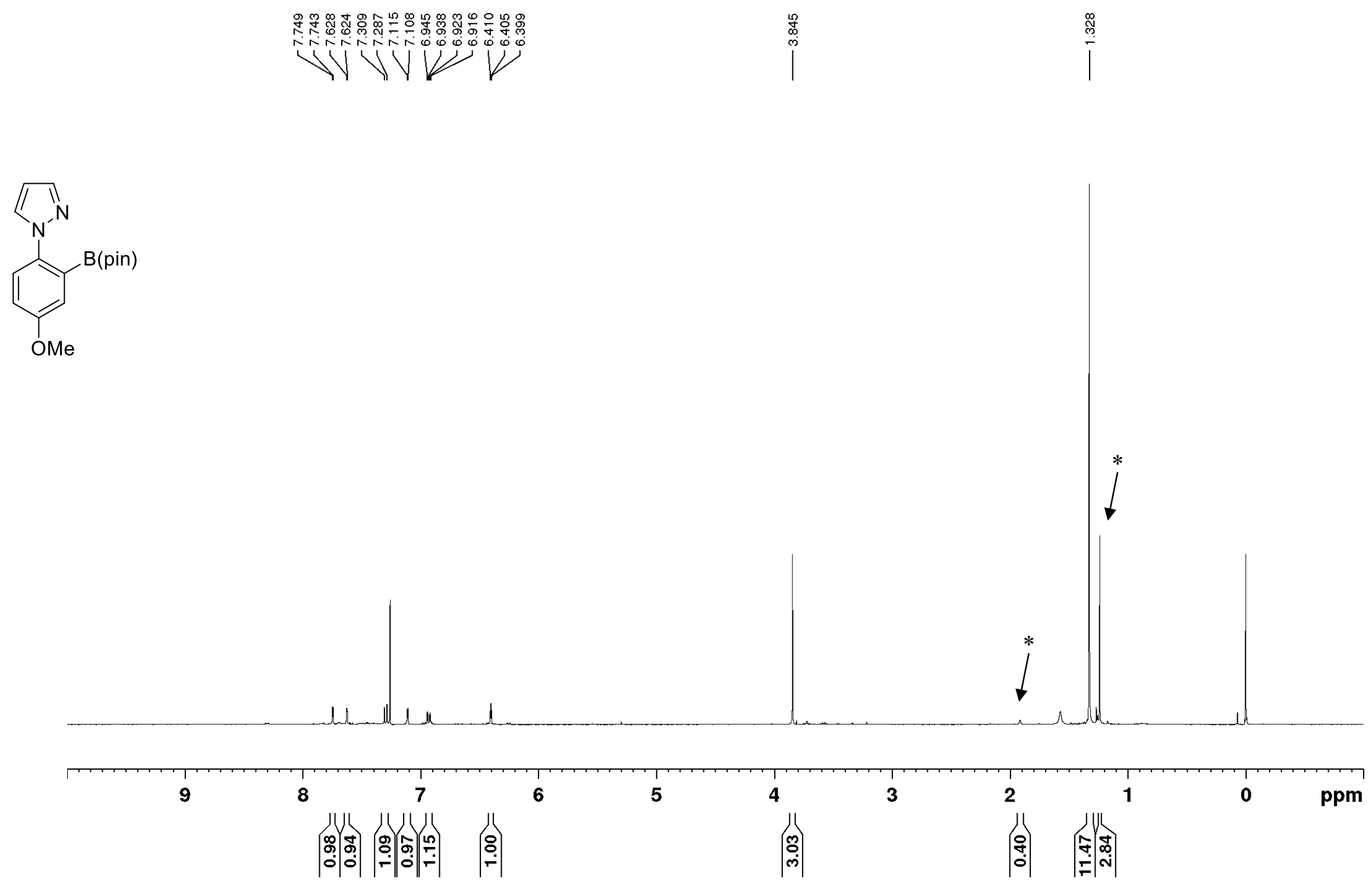
${ }^{13} \mathrm{C} \mathrm{NMR}\left(\mathrm{CDCl}_{3}, 100 \mathrm{MHz}\right)$ of 1-[4-Methoxy-2-(4,4,5,5-tetramethyl-1,3,2-dioxaborolan-2-yl)phenyl]-1H-pyrazole (3x)
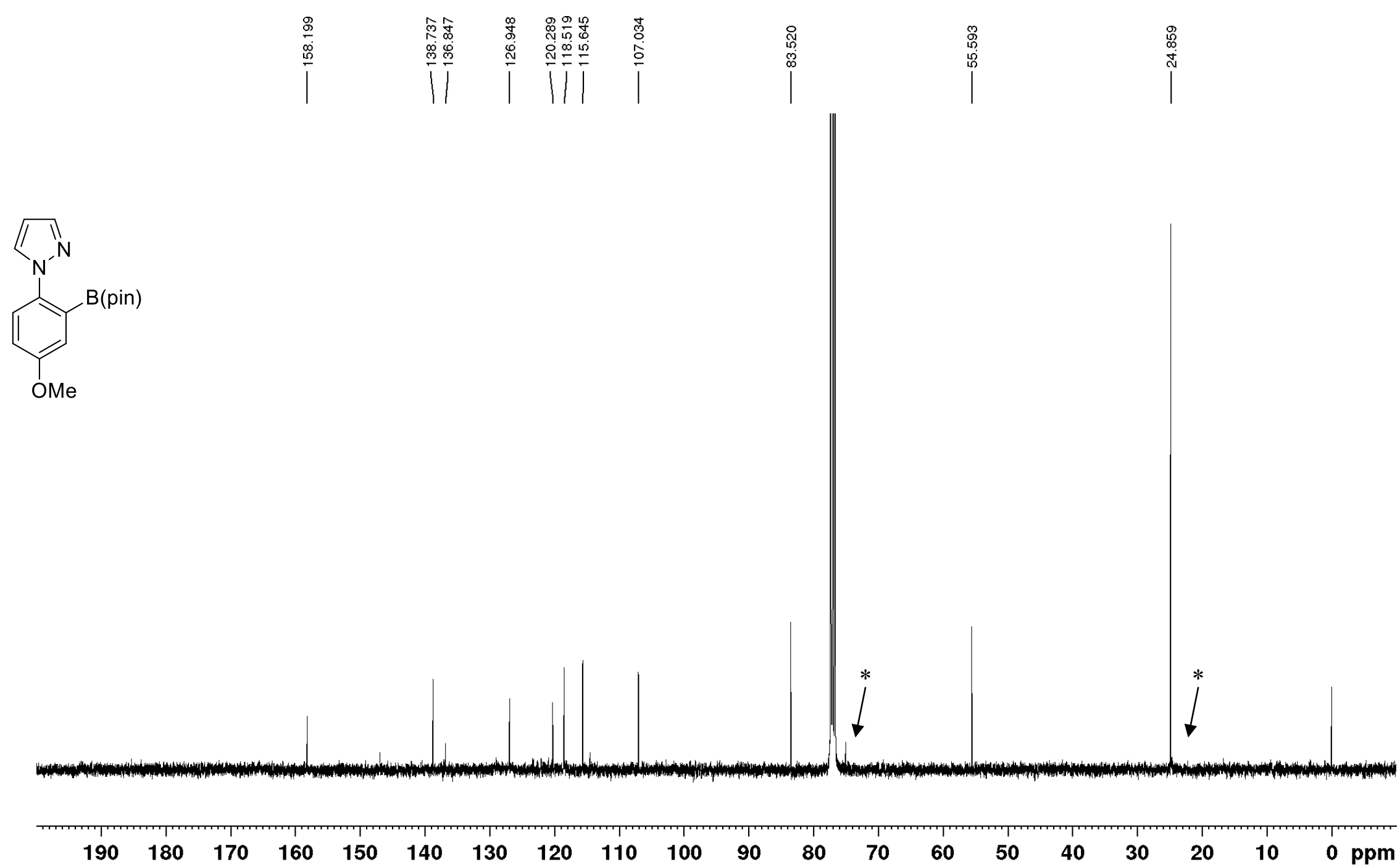
${ }^{1} \mathrm{H}$ NMR $\left(\mathrm{CDCl}_{3}, 400 \mathrm{MHz}\right)$ of 1-[4-(tert-Butyl)-2-(4,4,5,5-tetramethyl-1,3,2-dioxaborolan-2-yl)phenyl]-1H-pyrazole (3y)
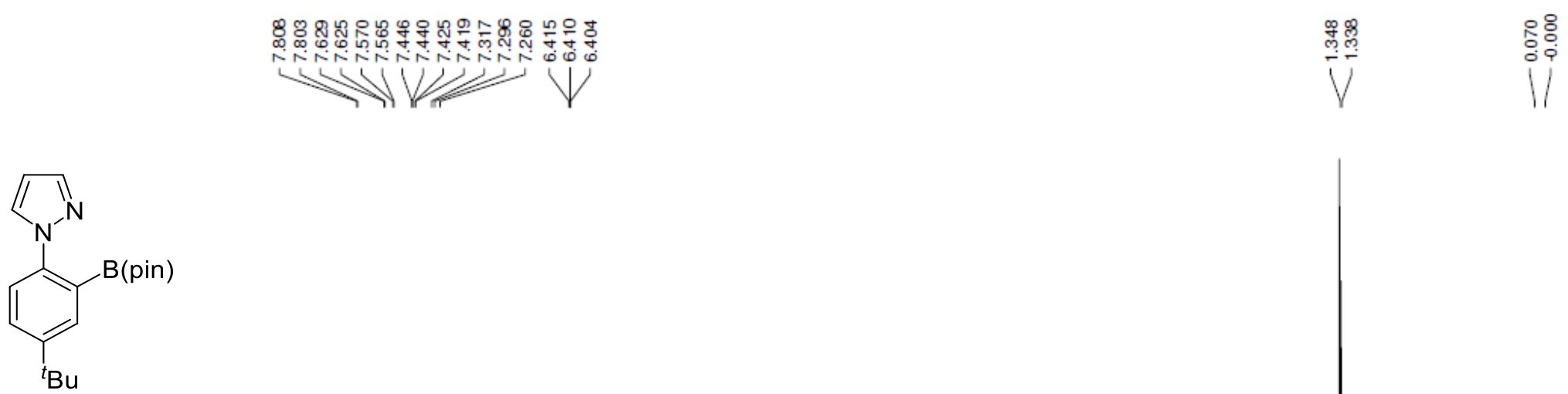

Bu

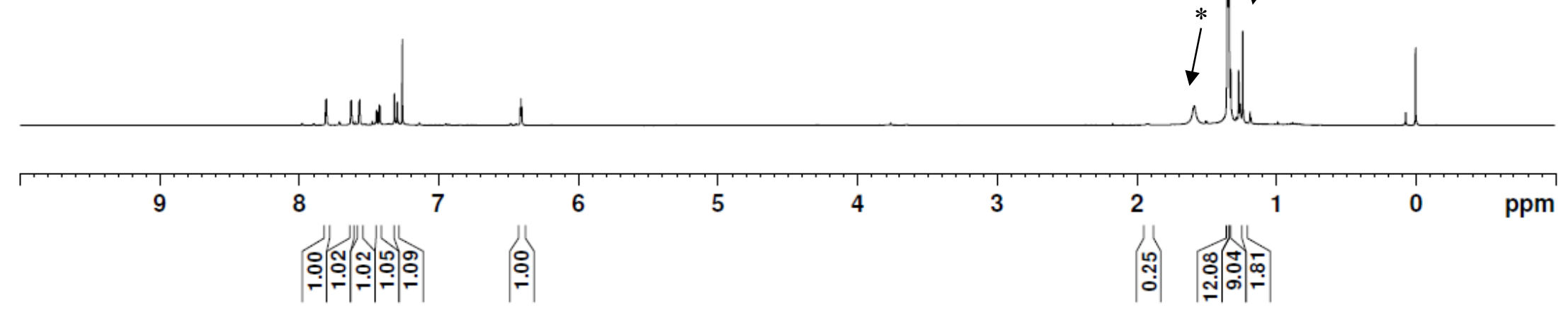


${ }^{13} \mathrm{C}$ NMR $\left(\mathrm{CDCl}_{3}, 100 \mathrm{MHz}\right)$ of 1-[4-(tert-Butyl)-2-(4,4,5,5-tetramethyl-1,3,2-dioxaborolan-2-yl)phenyl]-1H-pyrazole (3y)
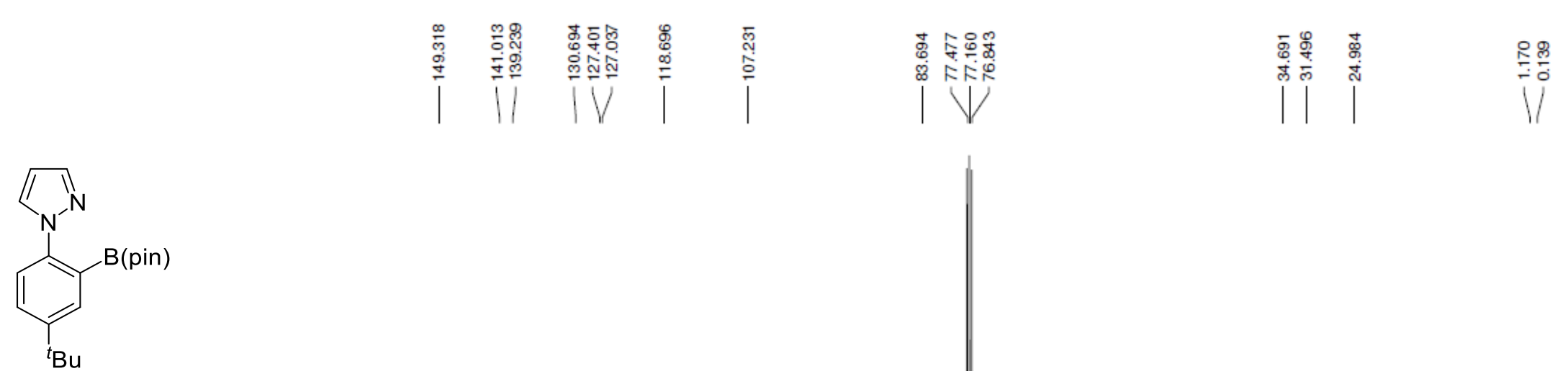

$$
\text { Bu }
$$

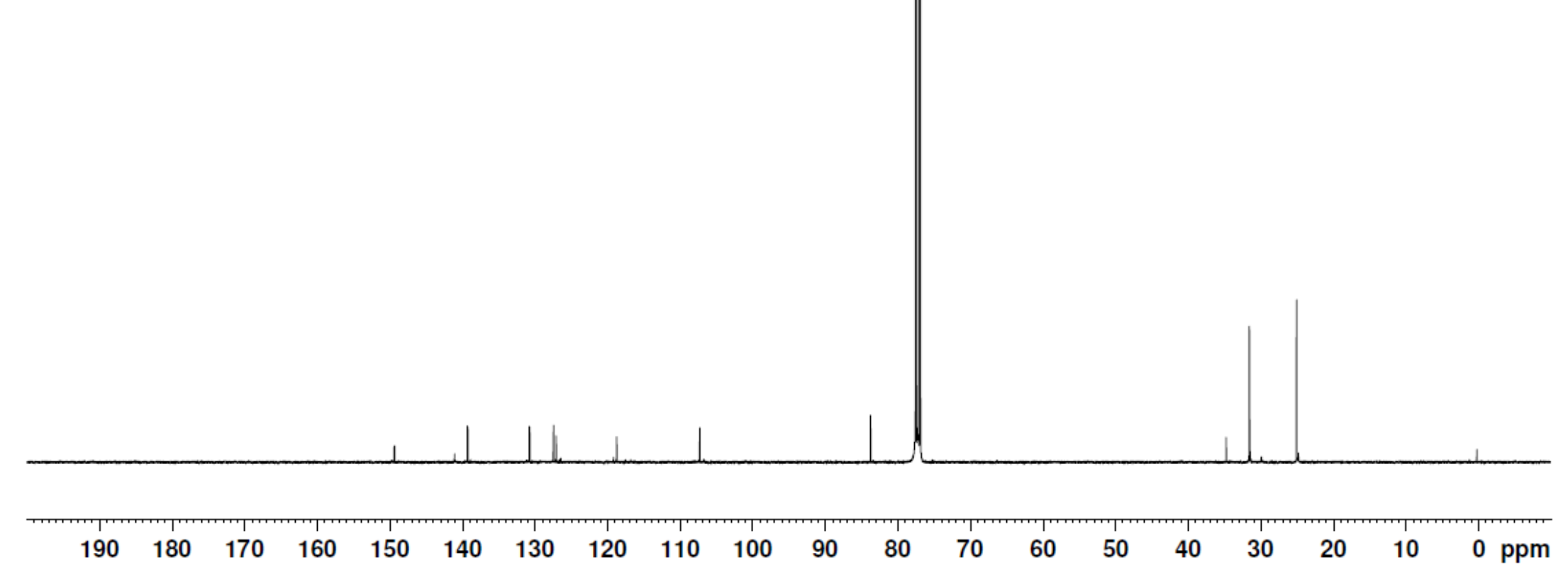


${ }^{1} \mathrm{H}$ NMR $\left(\mathrm{CDCl}_{3}, 400 \mathrm{MHz}\right)$ of 5-(tert-Butyl)-2-(1H-pyrazol-1-yl)phenol (4y)
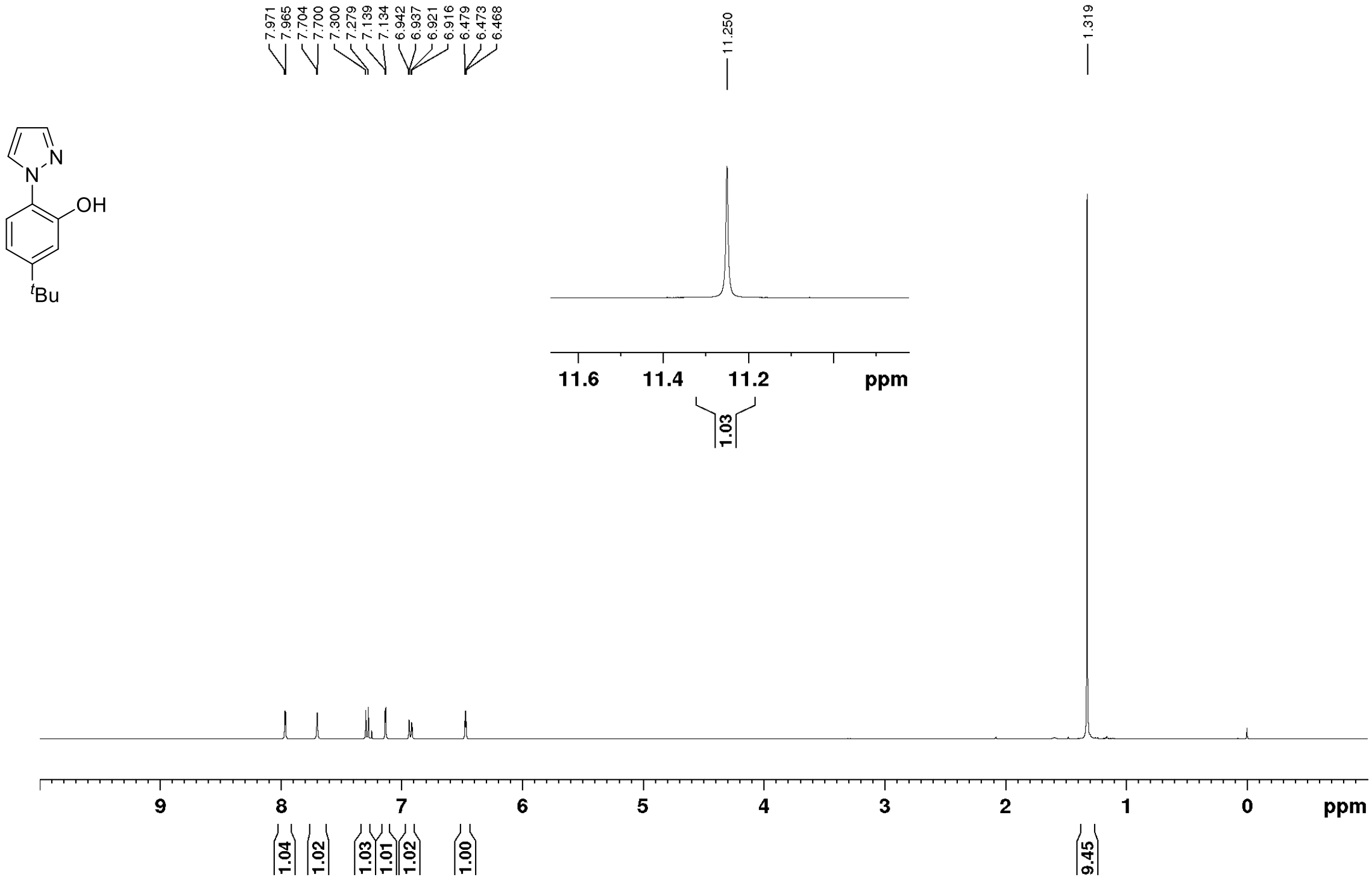
${ }^{13} \mathrm{C}$ NMR $\left(\mathrm{CDCl}_{3}, 100 \mathrm{MHz}\right)$ of 5-(tert-Butyl)-2-(1H-pyrazol-1-yl)phenol (4y)
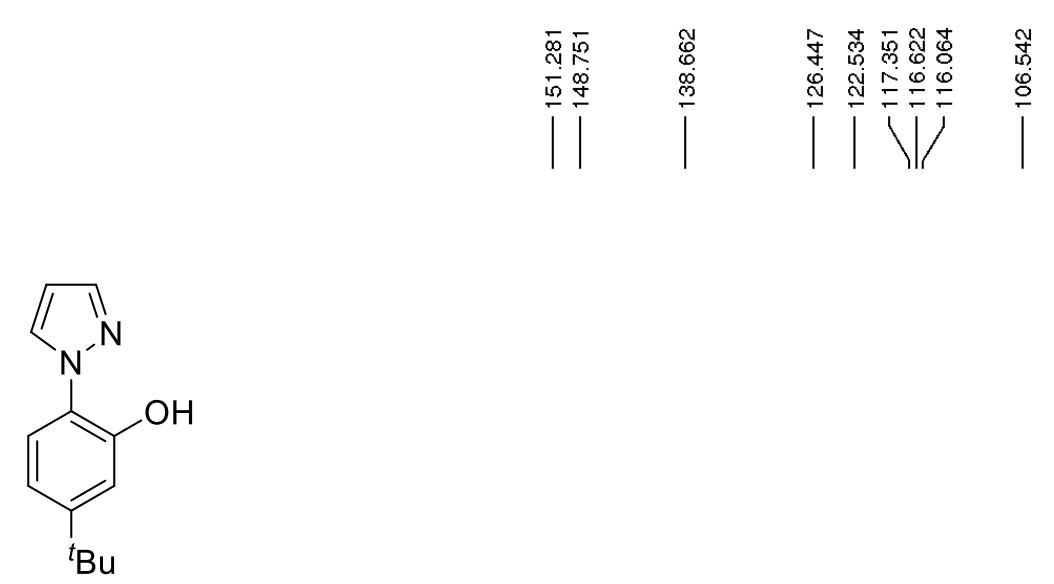

${ }^{t} \mathrm{Bu}$

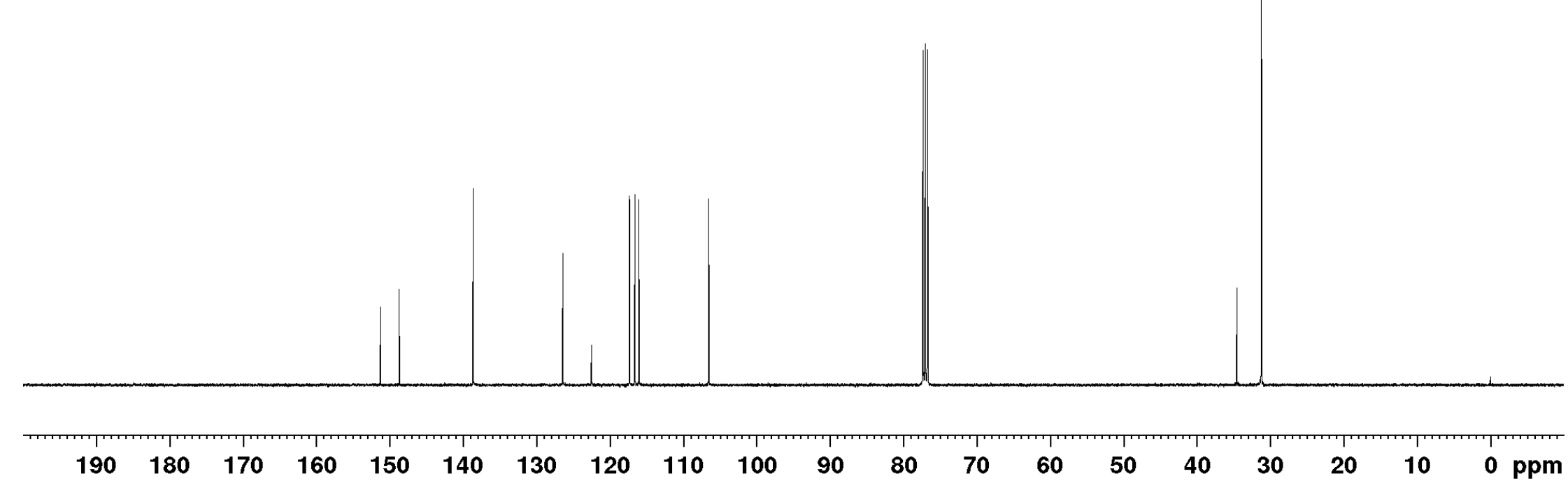


${ }^{1} \mathrm{H}$ NMR $\left(\mathrm{CDCl}_{3}, 400 \mathrm{MHz}\right)$ of 1-[2-(4,4,5,5-Tetramethyl-1,3,2-dioxaborolan-2-yl)naphthalen-1-yl]-1H-pyrazole (3z)

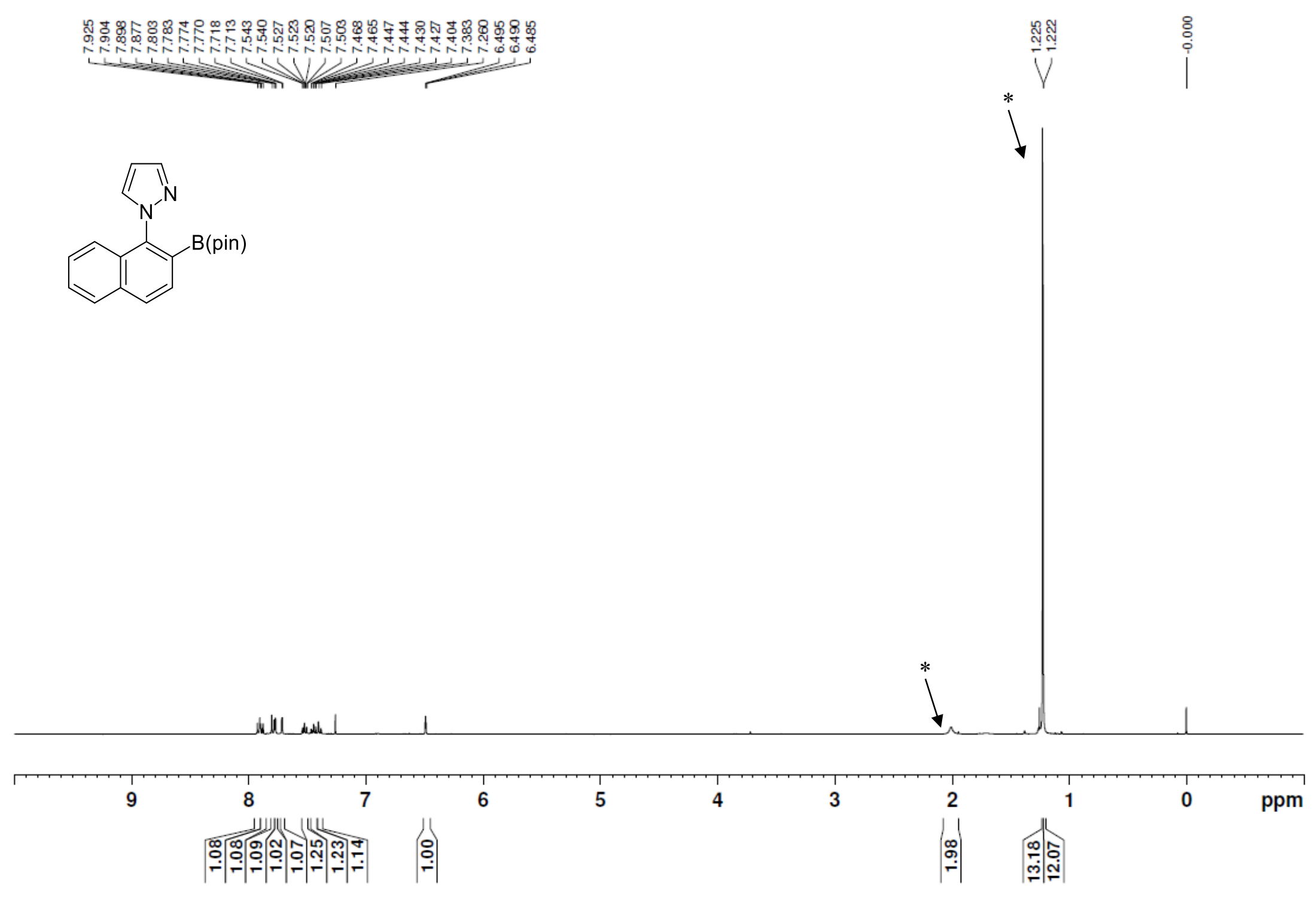


${ }^{13} \mathrm{C}$ NMR $\left(\mathrm{CDCl}_{3}, 100 \mathrm{MHz}\right)$ of 1-[2-(4,4,5,5-Tetramethyl-1,3,2-dioxaborolan-2-yl)naphthalen-1-yl]-1H-pyrazole (3z)

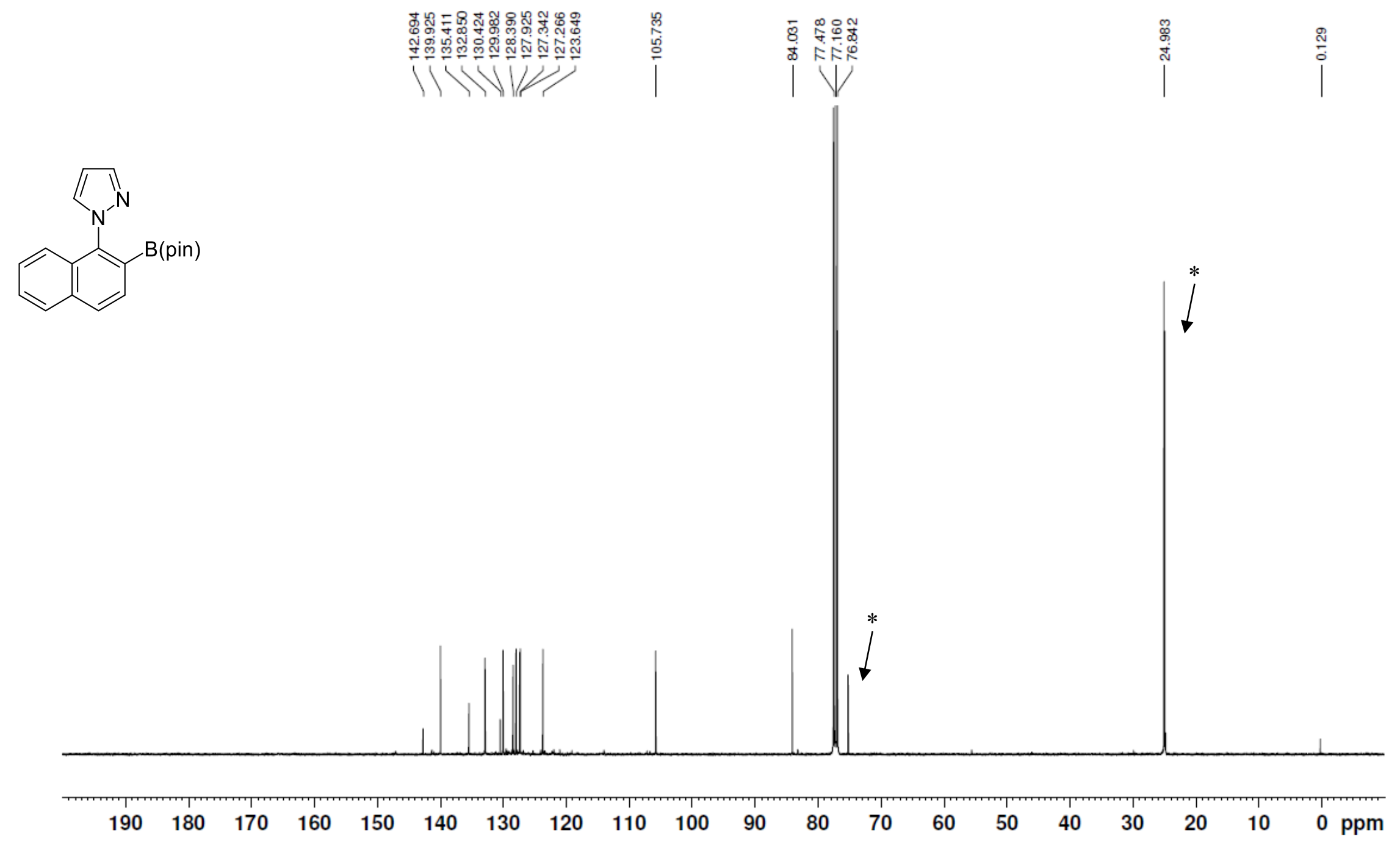


${ }^{1} \mathrm{H}$ NMR $\left(\mathrm{CDCl}_{3}, 400 \mathrm{MHz}\right)$ of 1-(1H-pyrazol-1-yl)naphthalen-2-ol (4z)

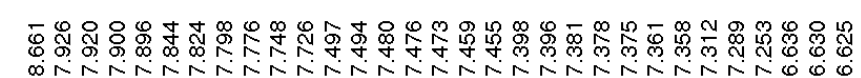

(

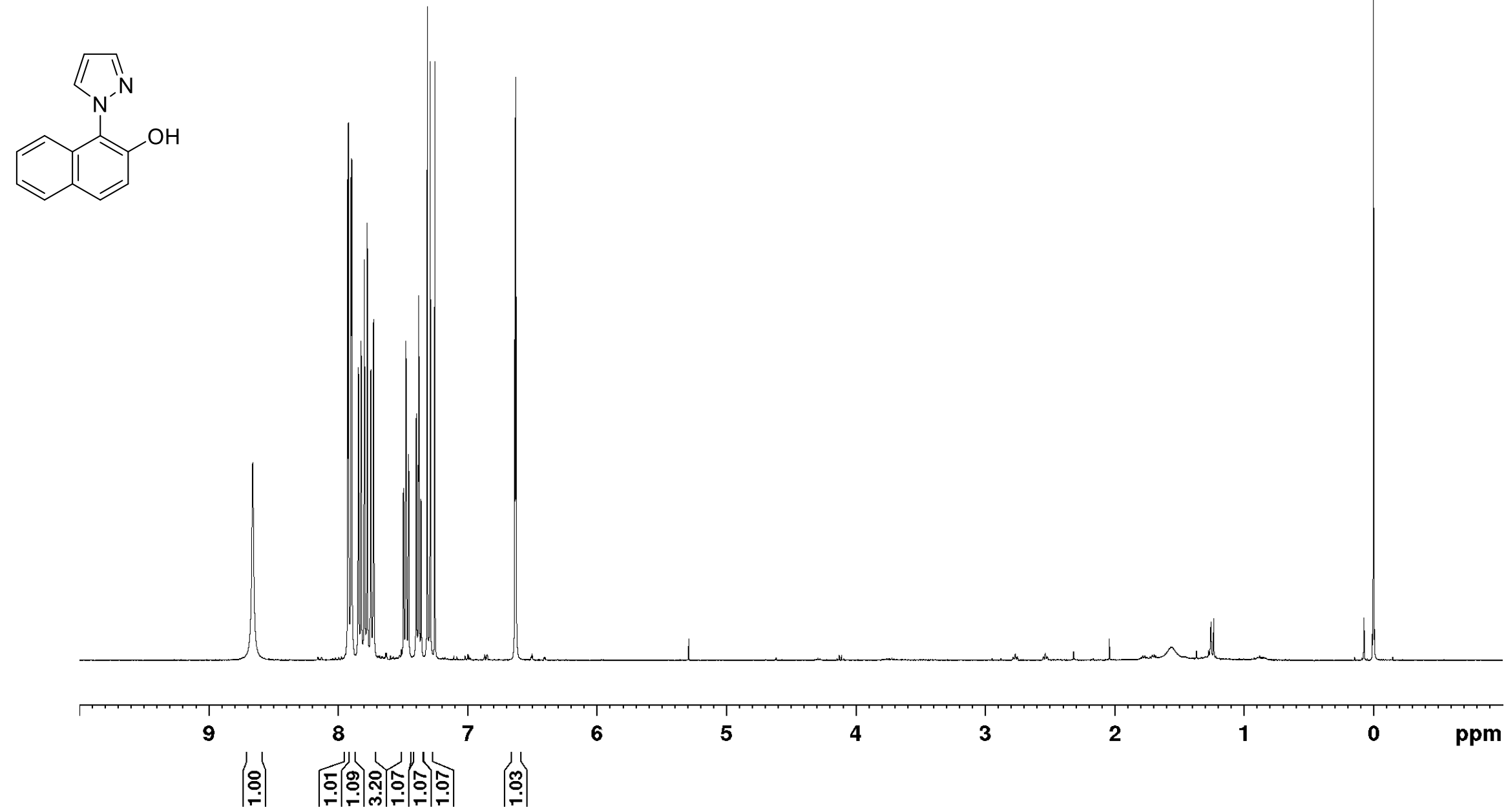


${ }^{13} \mathrm{C}$ NMR $\left(\mathrm{CDCl}_{3}, 100 \mathrm{MHz}\right)$ of 1-(1H-pyrazol-1-yl)naphthalen-2-ol (4z)
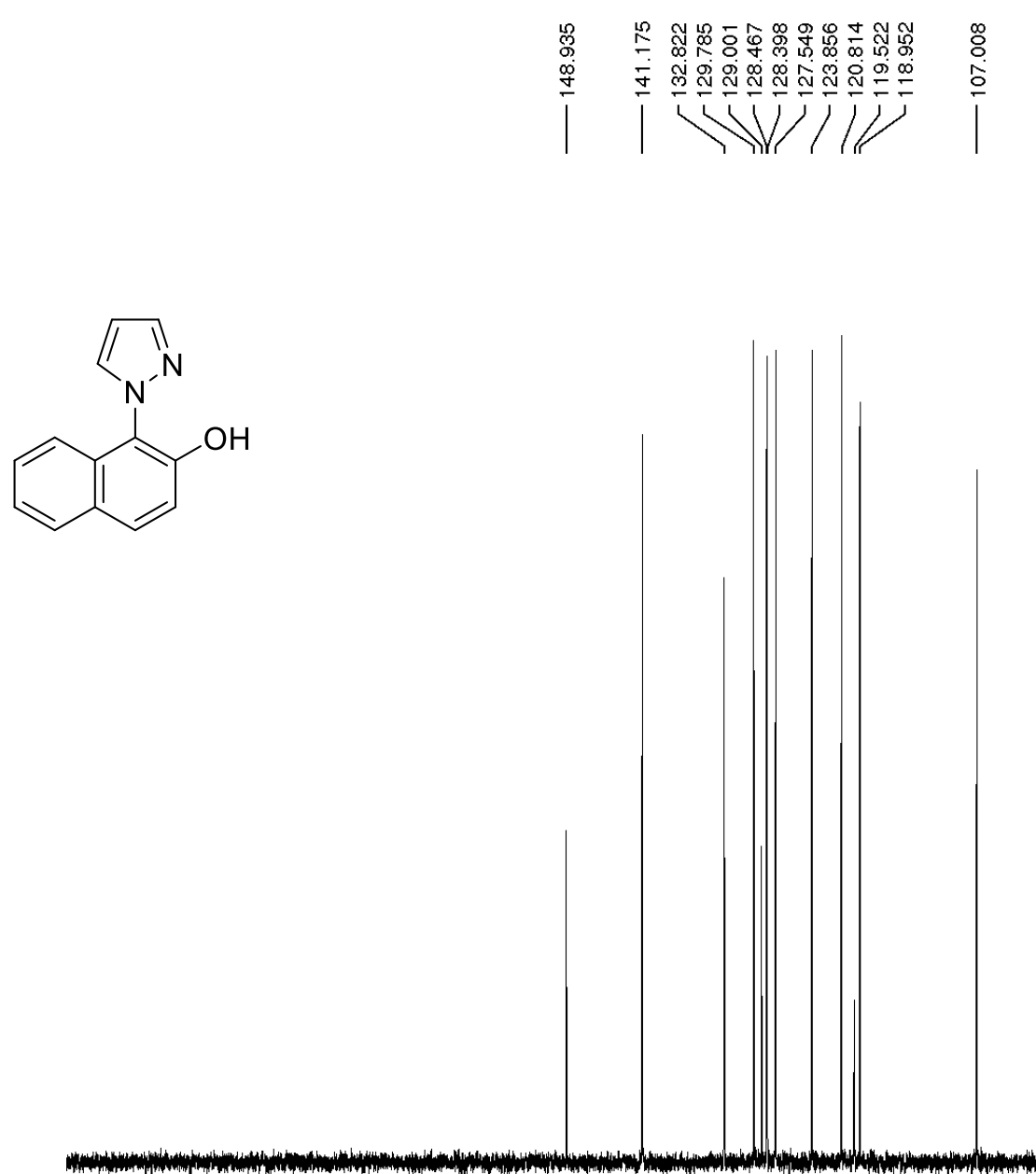

$\begin{array}{llllllllll}190 & 180 & 170 & 160 & 150 & 140 & 130 & 120 & 110 & 100\end{array}$

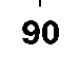

80

7060

50

40

30

20

10

0 ppm 
${ }^{1} \mathrm{H}$ NMR $\left(\mathrm{CDCl}_{3}, 400 \mathrm{MHz}\right)$ of 2-\{[Benzyl(methyl)amino]methyl $\}$ phenol (6a)

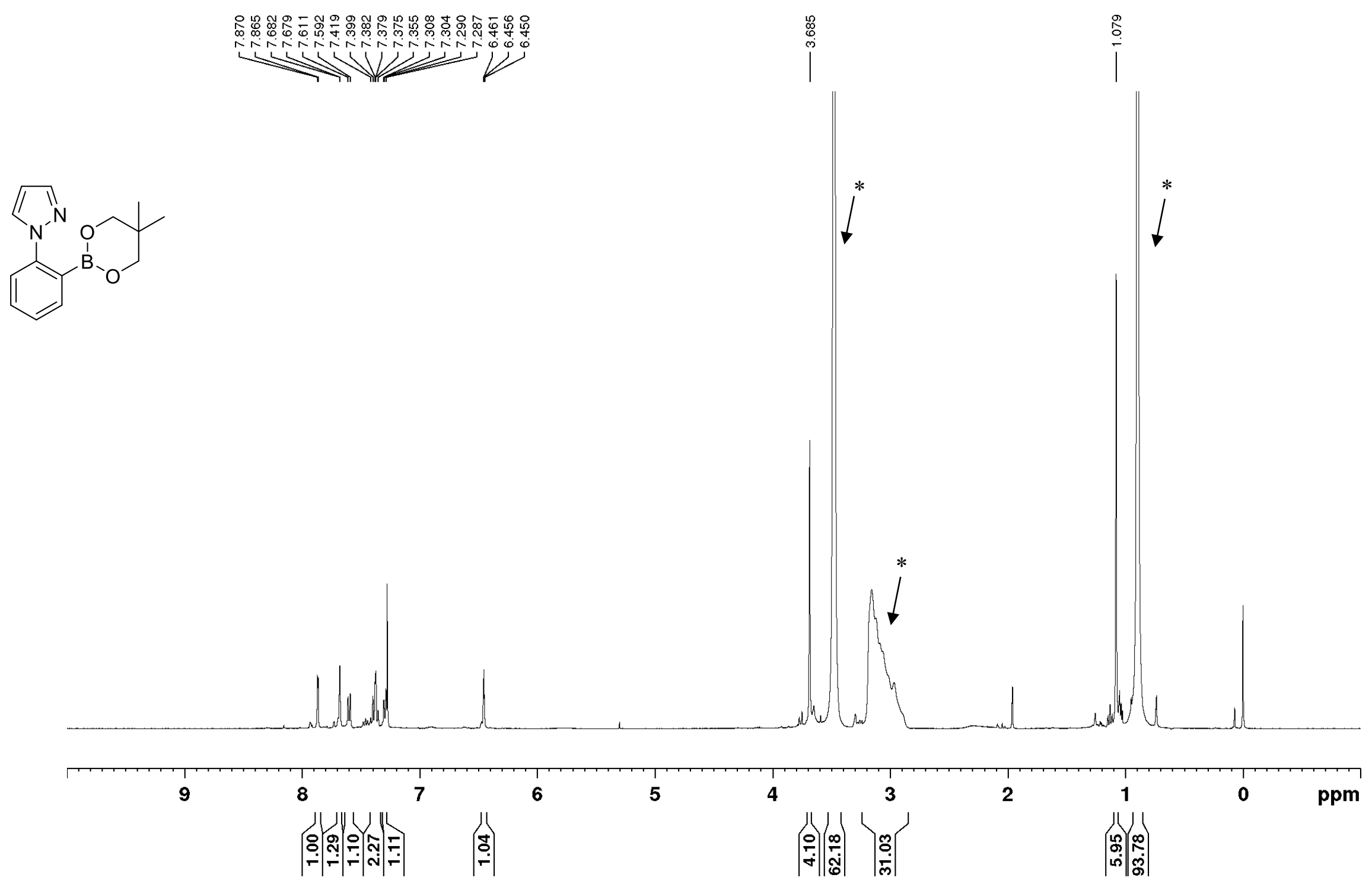


${ }^{13} \mathrm{C}$ NMR $\left(\mathrm{CDCl}_{3}, 100 \mathrm{MHz}\right)$ of 2-\{[Benzyl(methyl)amino]methyl $\}$ phenol (6a)

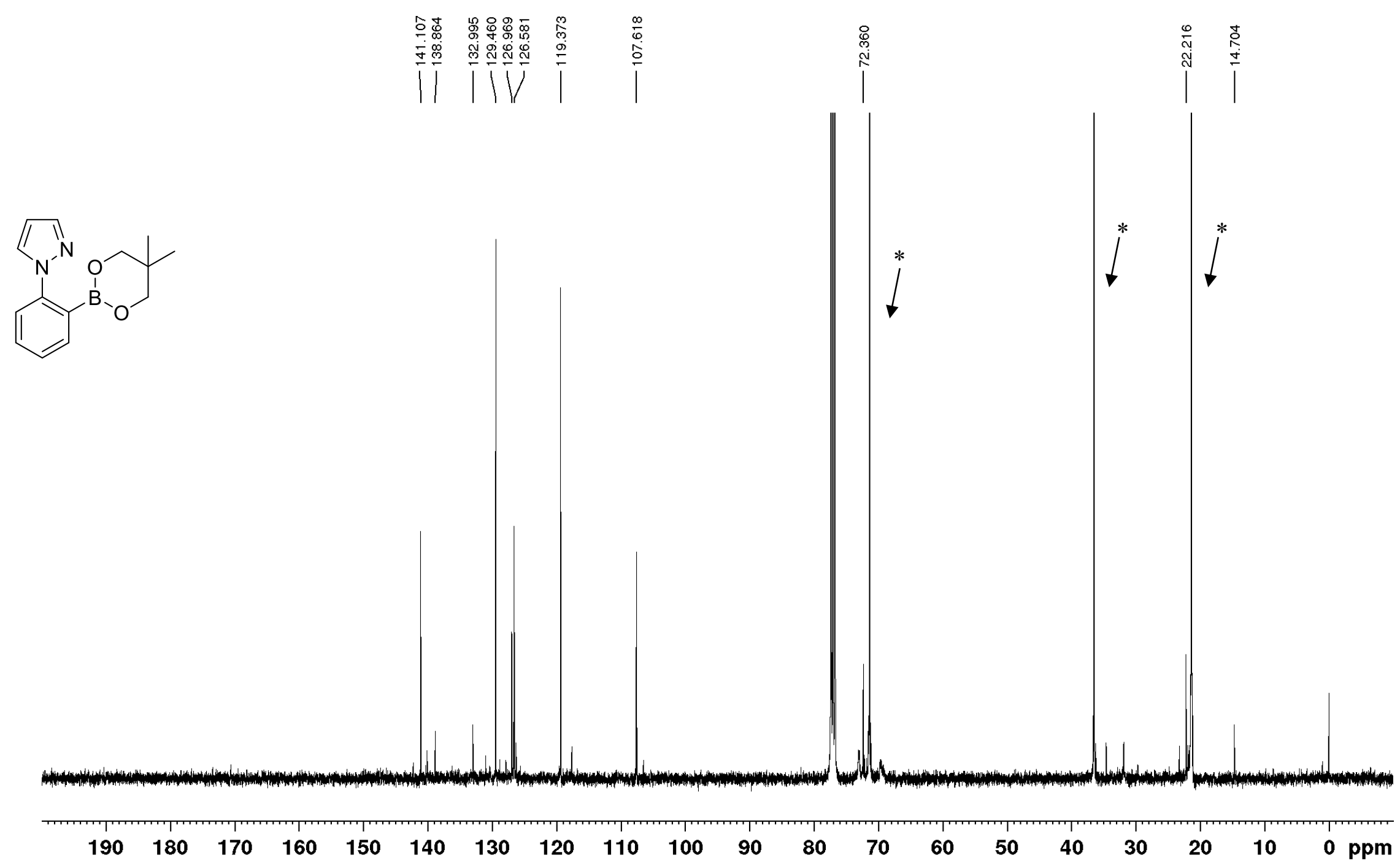


${ }^{1} \mathrm{H}$ NMR $\left(\mathrm{CDCl}_{3}, 400 \mathrm{MHz}\right)$ of Methyl 4-(1H-pyrazol-1-yl)-3-(4,4,5,5-tetramethyl-1,3,2-dioxaborolan-2-yl)benzoate (8)

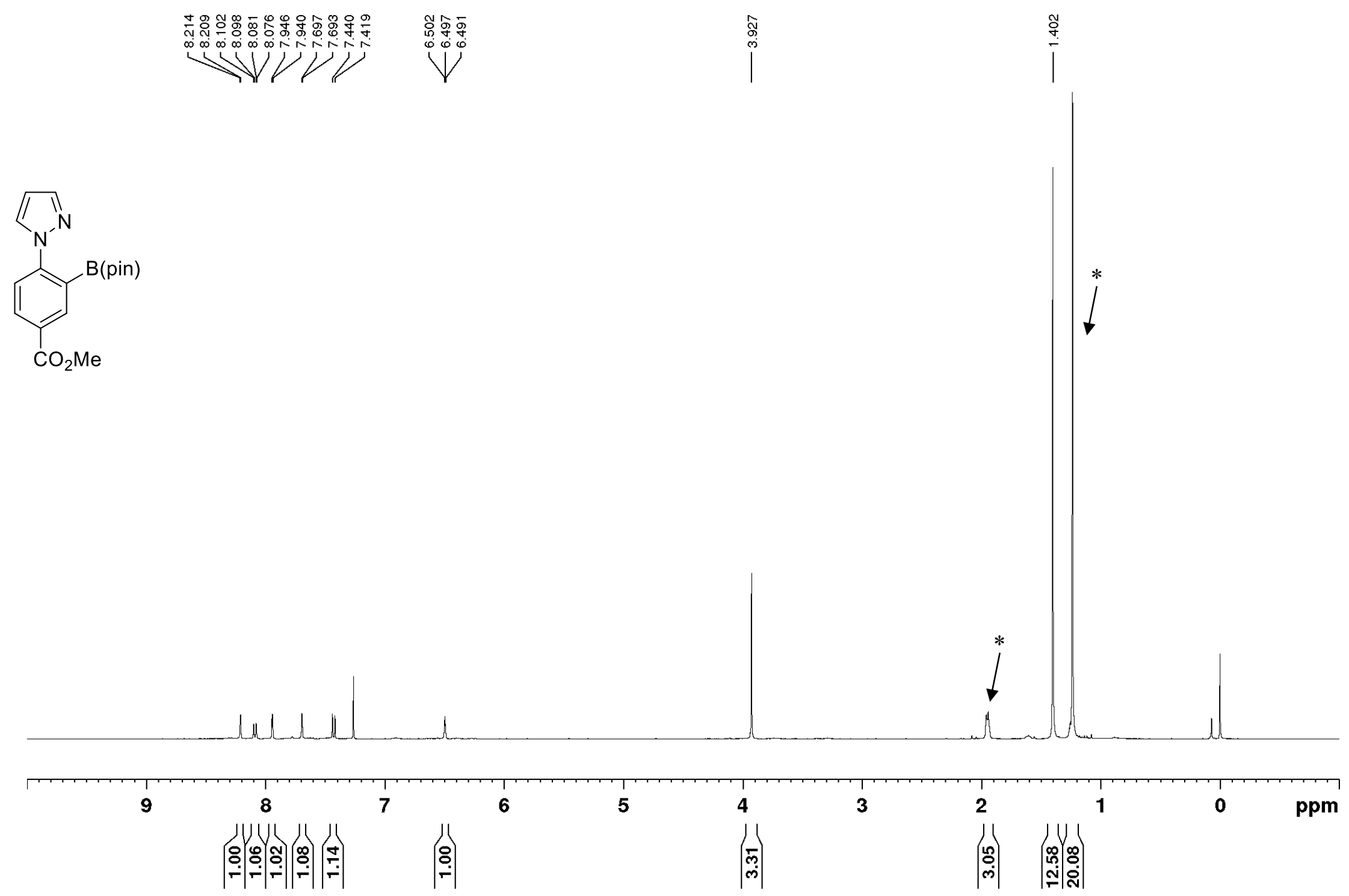


${ }^{13} \mathrm{C}$ NMR $\left(\mathrm{CDCl}_{3}, 100 \mathrm{MHz}\right)$ of Methyl 4-(1H-pyrazol-1-yl)-3-(4,4,5,5-tetramethyl-1,3,2-dioxaborolan-2-yl)benzoate (8)

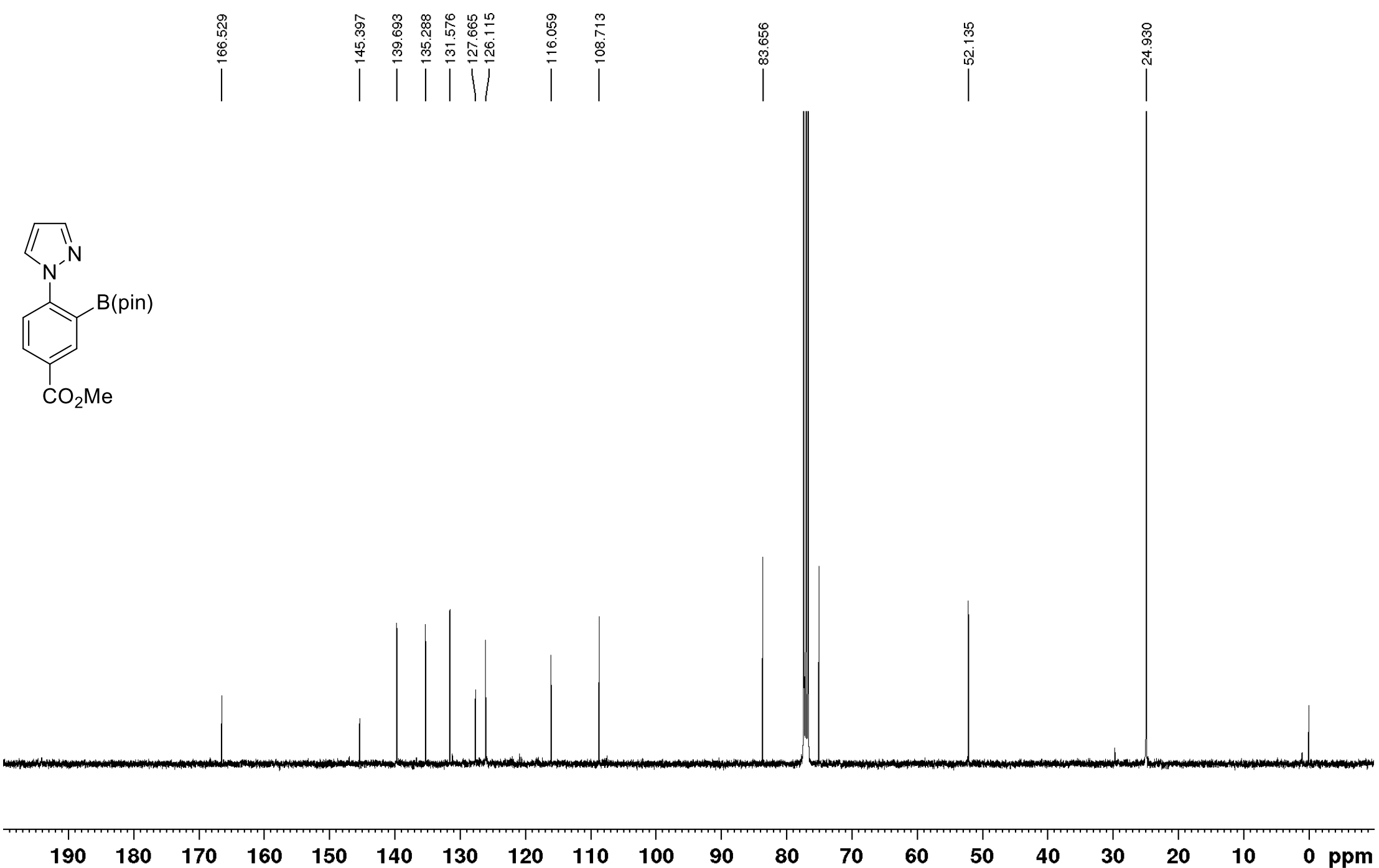


${ }^{1} \mathrm{H}$ NMR $\left(\mathrm{CDCl}_{3}, 400 \mathrm{MHz}\right)$ of 1-(2-Chlorophenyl)-3,5-dimethyl-1H-pyrazole (9b)
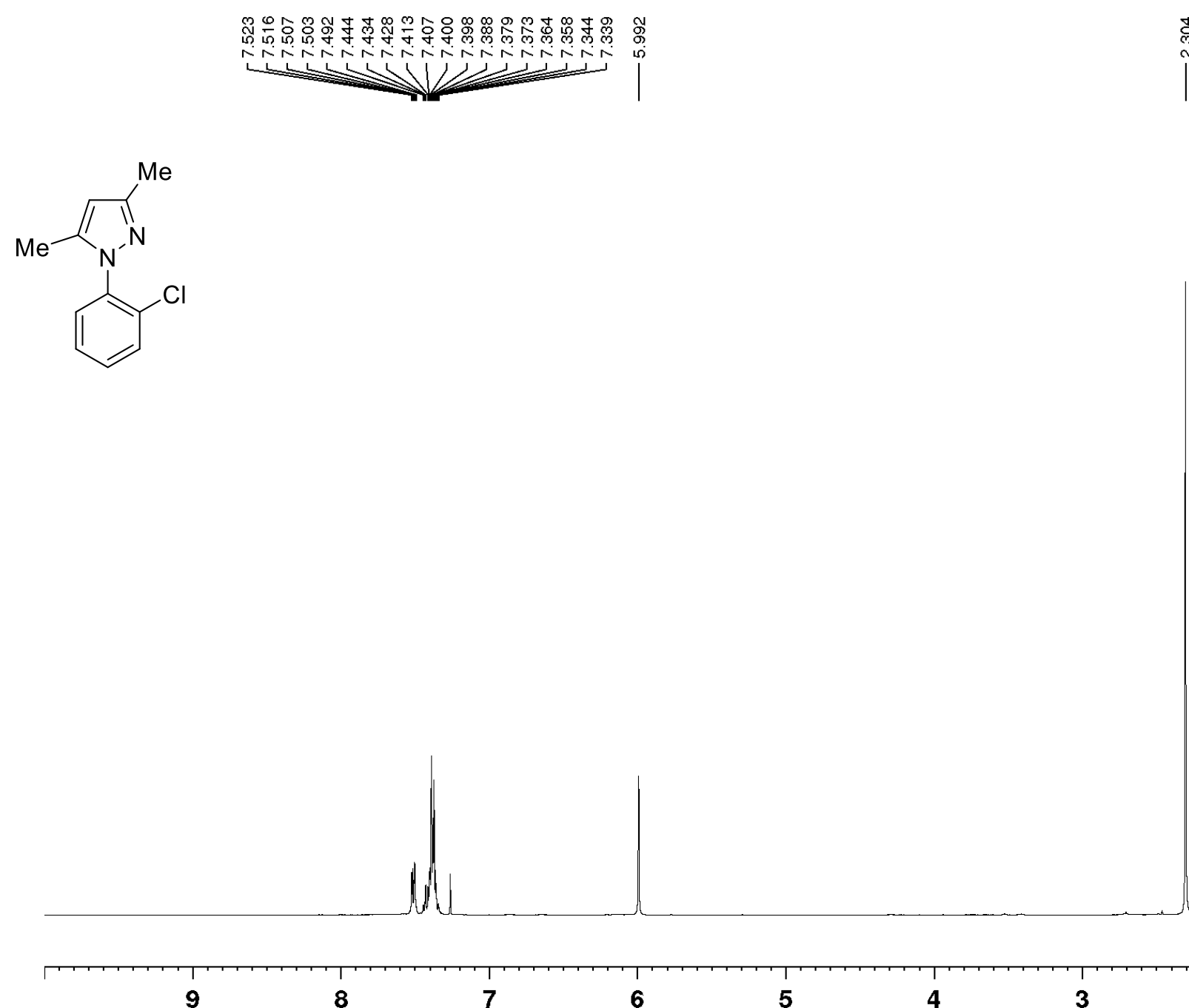

8

7

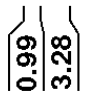

6

5

$\left|\begin{array}{l}0 \\ 0 \\ \hdashline\end{array}\right|$

4

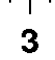

2

oो

ले

ppm 
${ }^{13} \mathrm{C}$ NMR $\left(\mathrm{CDCl}_{3}, 100 \mathrm{MHz}\right)$ of 1-(2-Chlorophenyl)-3,5-dimethyl-1H-pyrazole (9b)

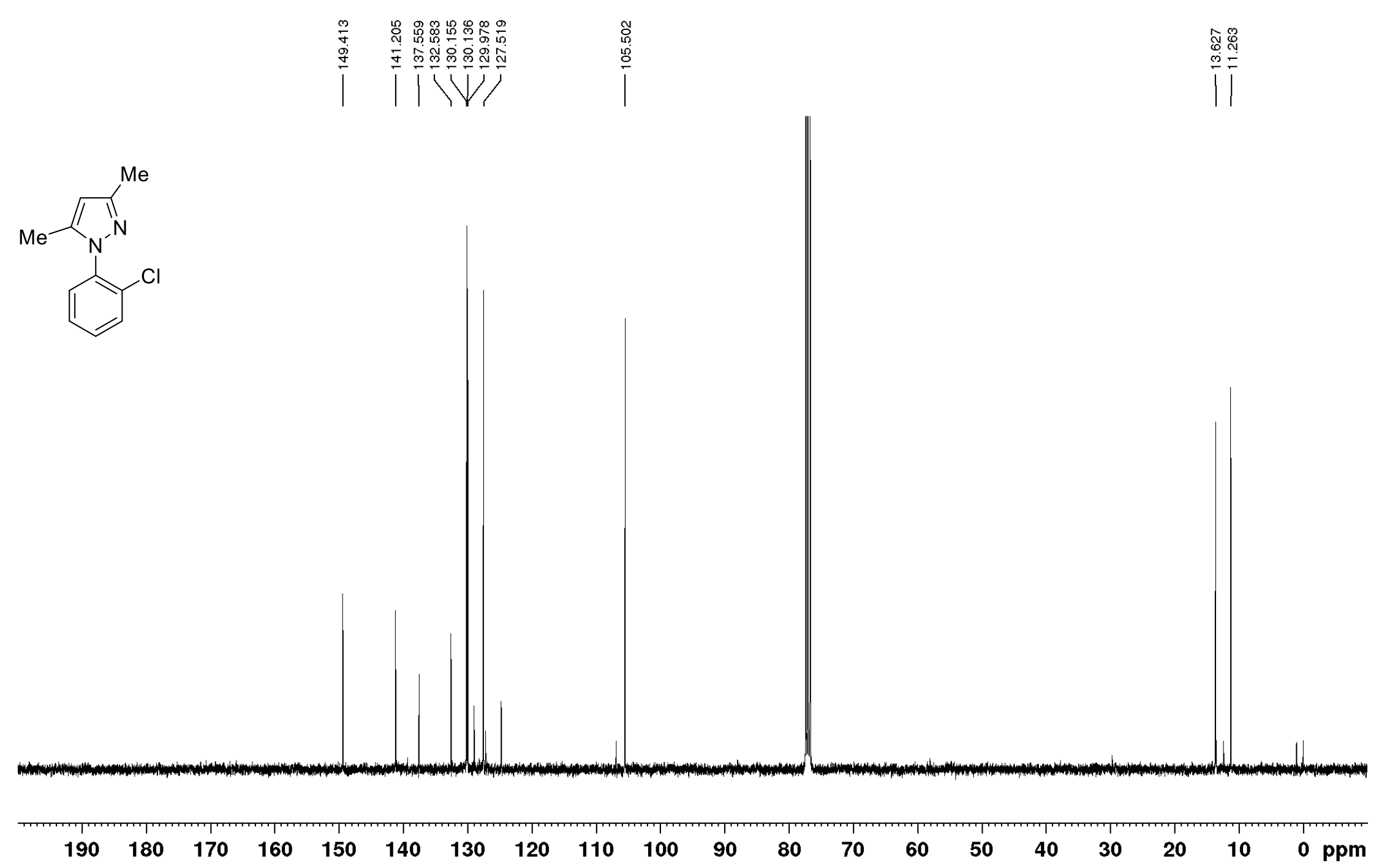


${ }^{1} \mathrm{H}$ NMR $\left(\mathrm{CDCl}_{3}, 400 \mathrm{MHz}\right)$ of 3,5-Dimethyl-1-(phenyl-2- $\left.d\right)$-1H-pyrazole (10b)
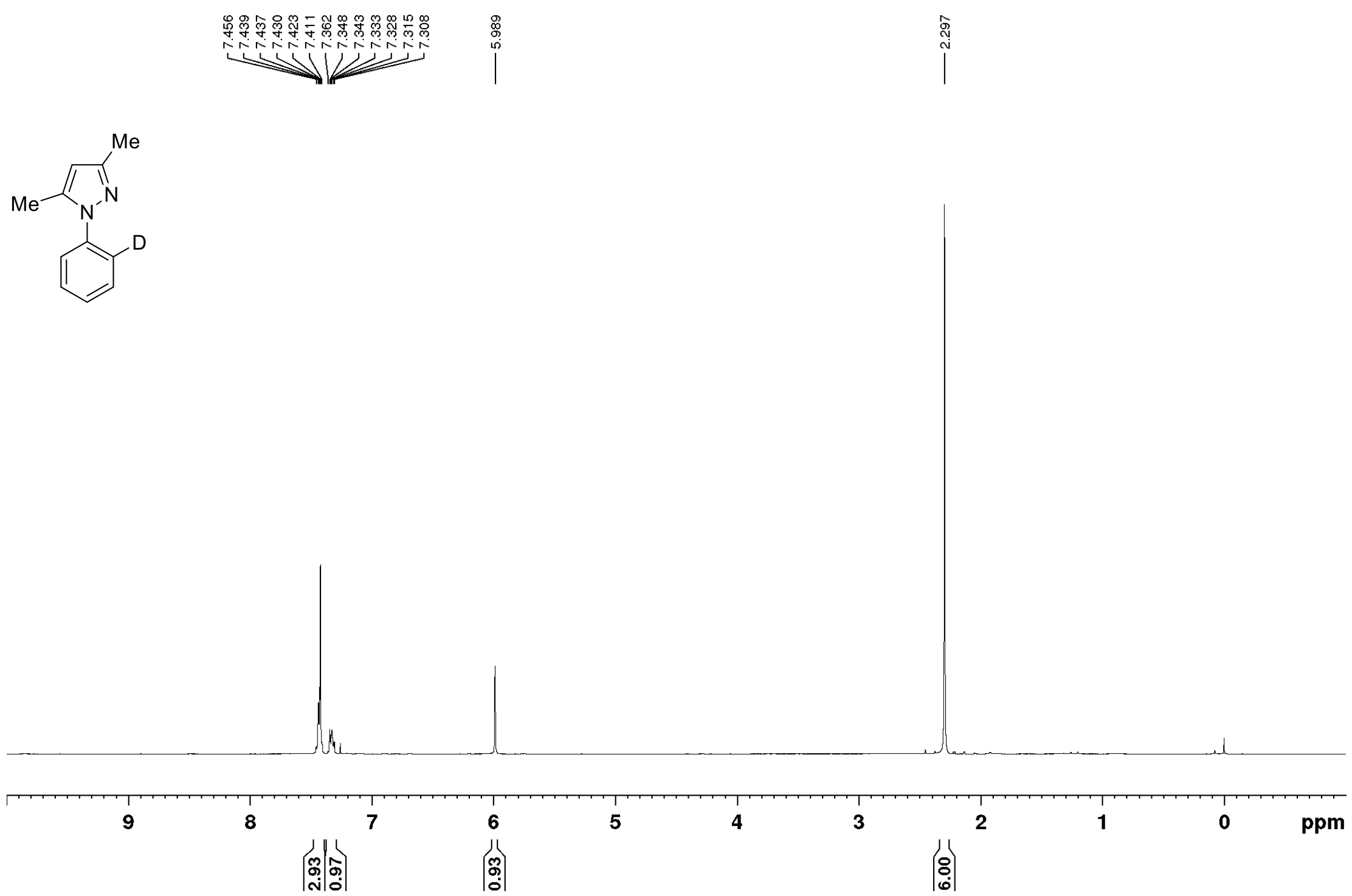
${ }^{13} \mathrm{C}$ NMR $\left(\mathrm{CDCl}_{3}, 100 \mathrm{MHz}\right)$ of 3,5-Dimethyl-1-(phenyl-2- $d$ )- $1 \mathrm{H}$-pyrazole (10b)
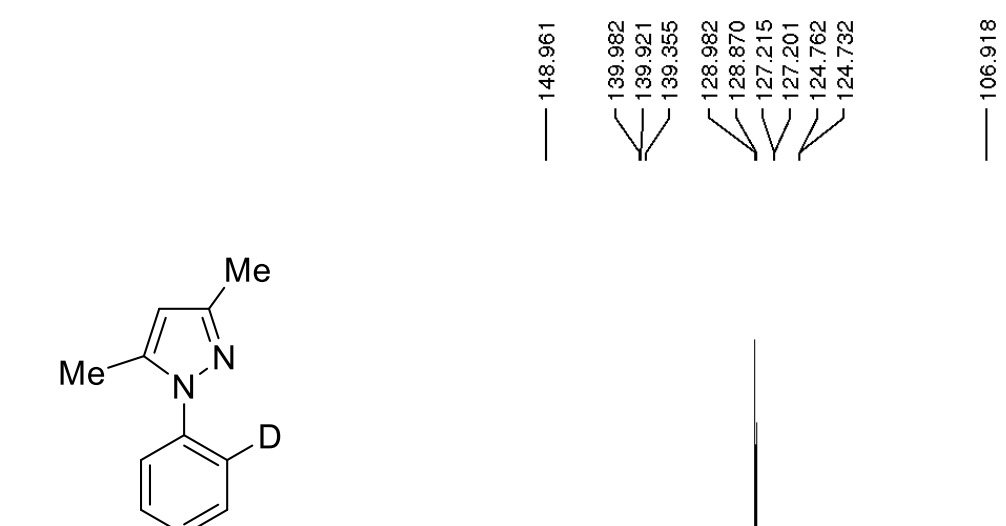

$\left.\right|^{\infty}$

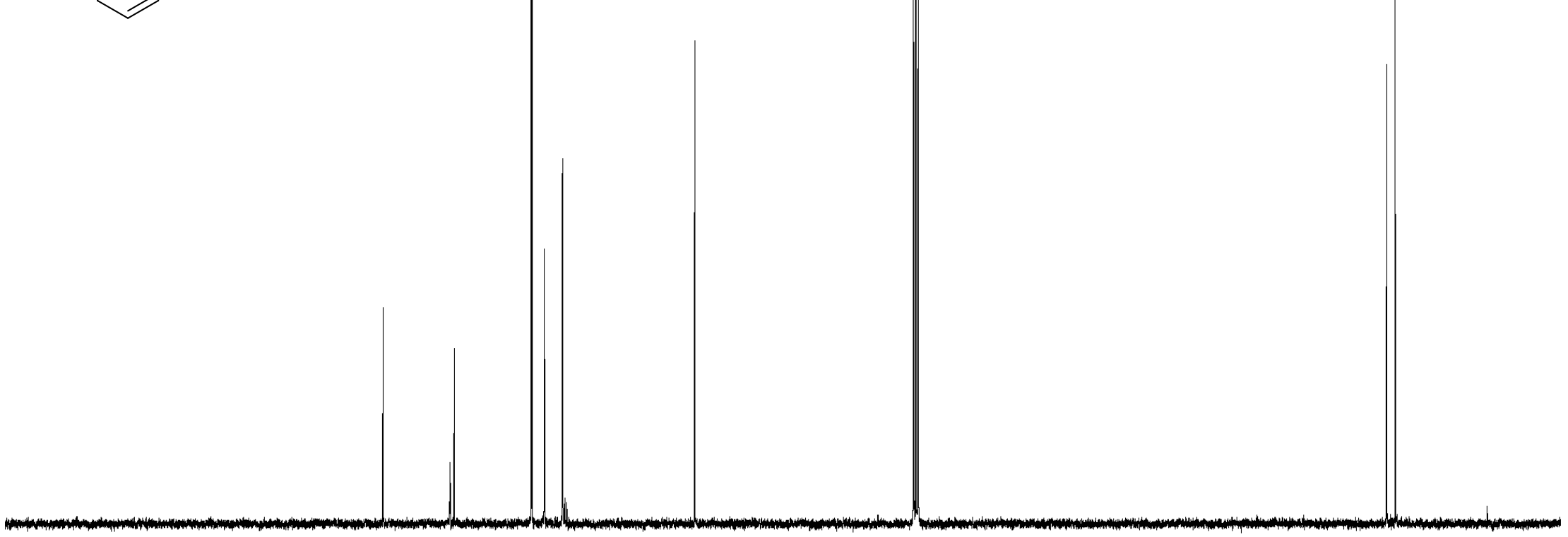

$\begin{array}{llllllllll}190 & 180 & 170 & 160 & 150 & 140 & 130 & 120 & 110 & 100\end{array}$

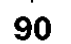

80

7060

50

40

30

20

10

0 ppm 
${ }^{1} \mathrm{H}$ NMR $\left(\mathrm{CDCl}_{3}, 400 \mathrm{MHz}\right)$ of 3,5-Dimethyl-1-\{2-[(triisopropylsilyl)ethynyl]phenyl $\}-1 \mathrm{H}$-pyrazole (11b)

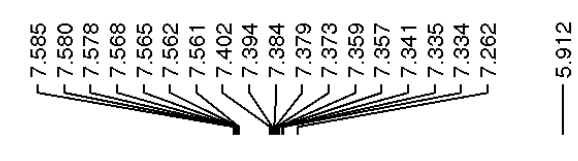

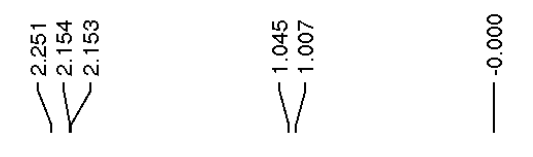

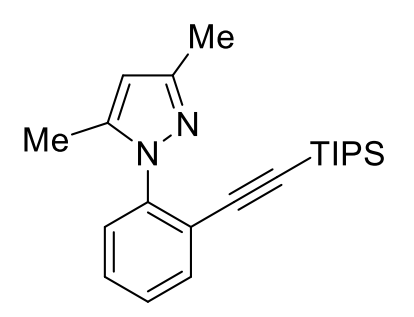

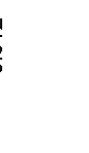

(n)

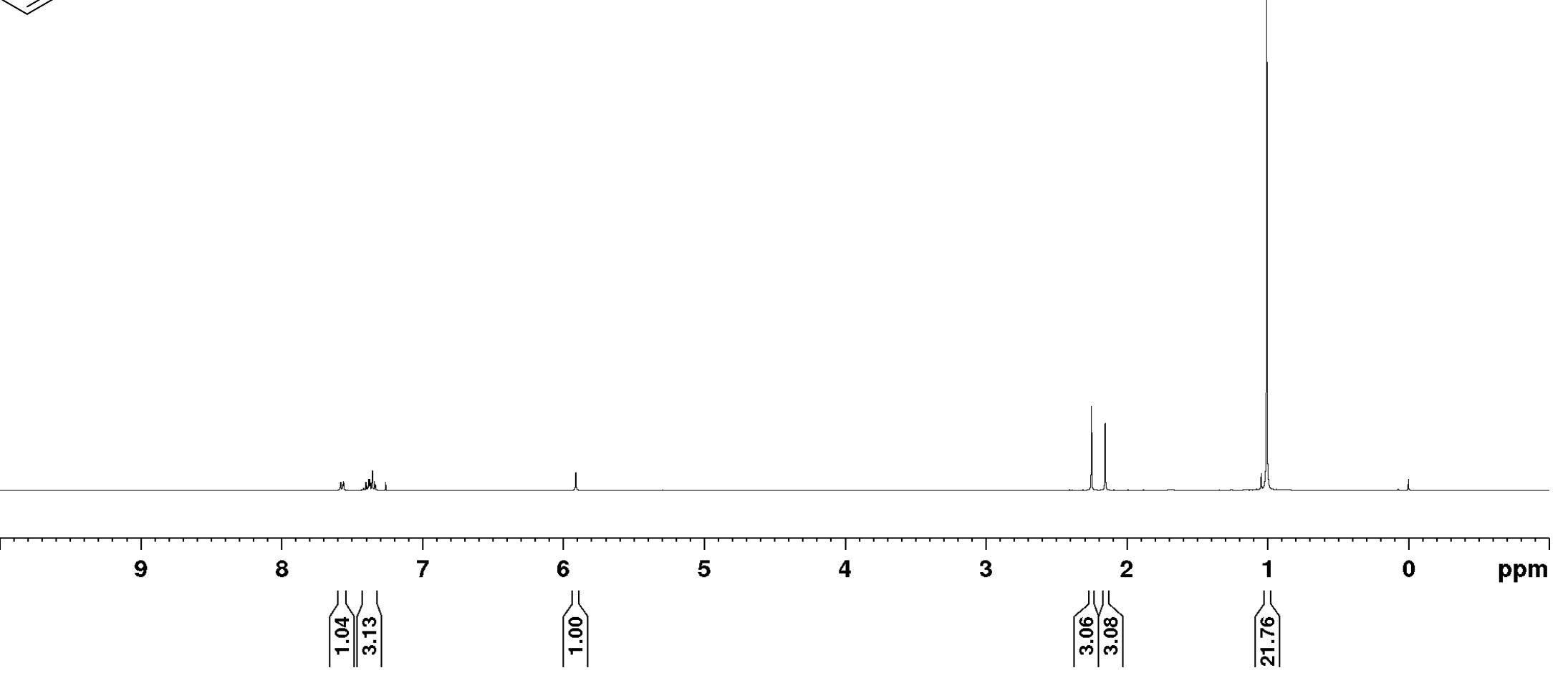


${ }^{13} \mathrm{C}$ NMR $\left(\mathrm{CDCl}_{3}, 100 \mathrm{MHz}\right)$ of 3,5-Dimethyl-1-\{2-[(triisopropylsilyl)ethynyl]phenyl $\}$ - $1 \mathrm{H}$-pyrazole (11b)
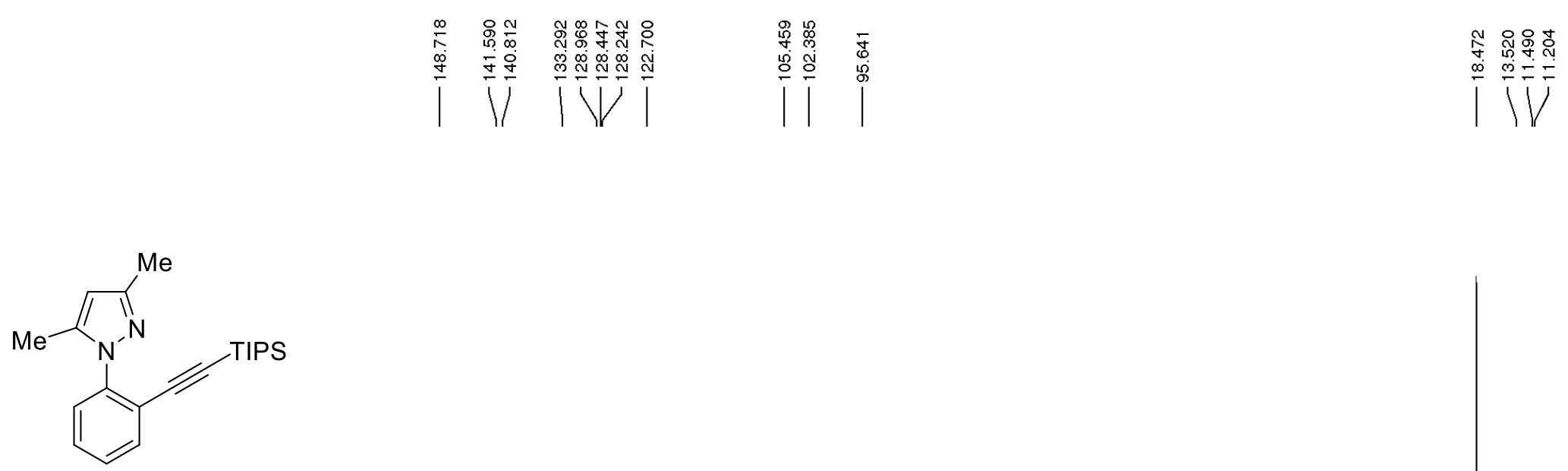

$\mid$

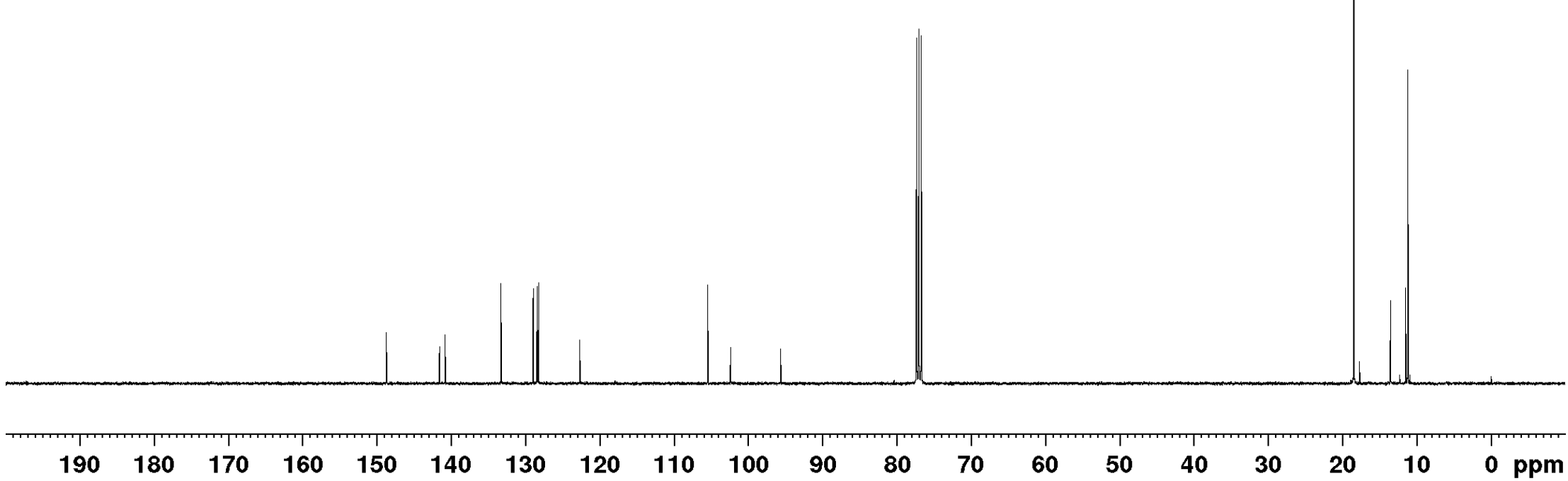


${ }^{1} \mathrm{H}$ NMR $\left(\mathrm{CDCl}_{3}, 400 \mathrm{MHz}\right)$ of 1-\{4'-Methoxy-[1,1'-biphenyl]-2-yl\}-3,5-dimethyl-1H-pyrazole (12b)

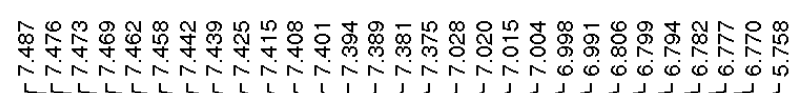

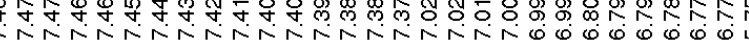

(1)
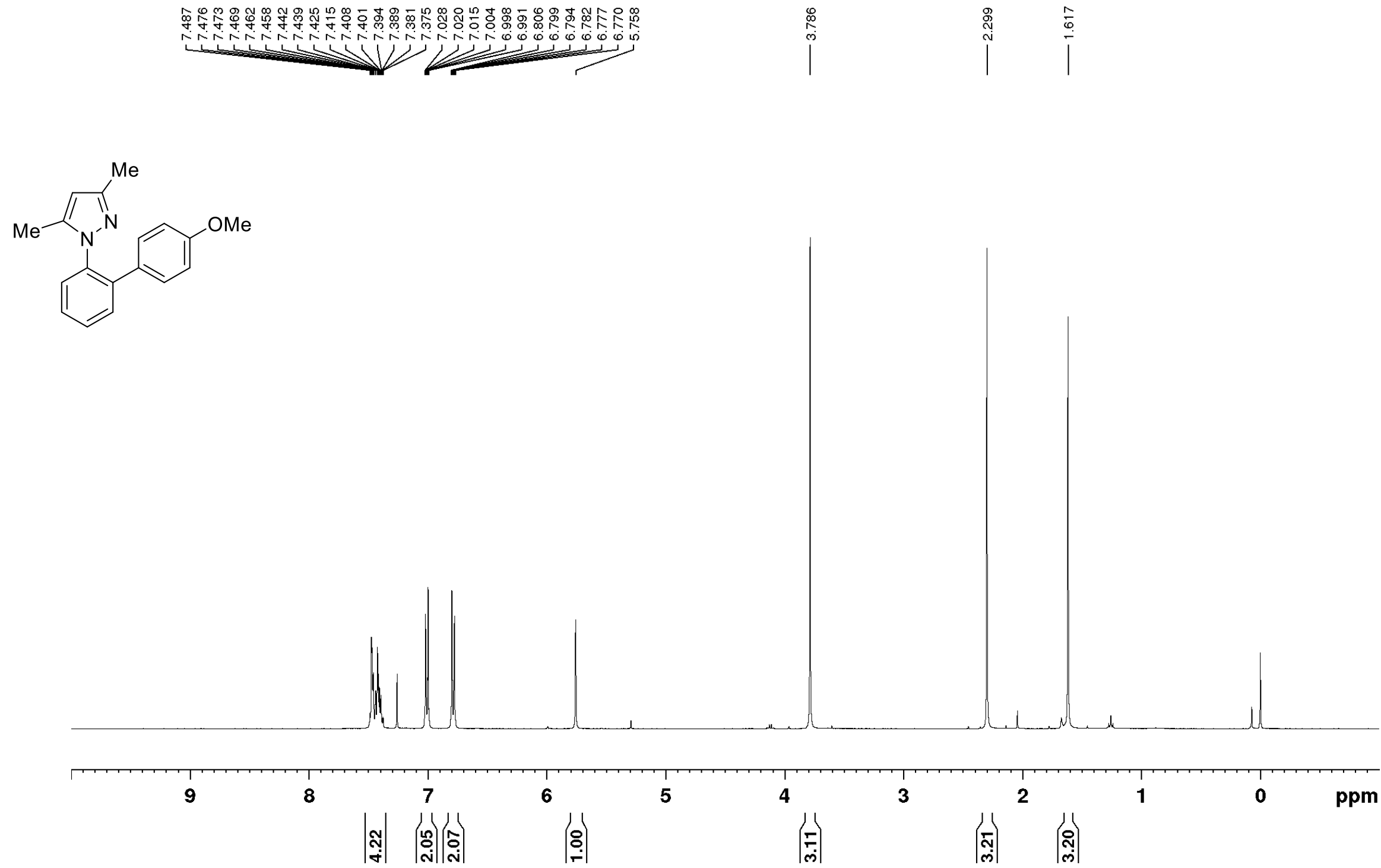
${ }^{13} \mathrm{C}$ NMR $\left(\mathrm{CDCl}_{3}, 100 \mathrm{MHz}\right)$ of 1-\{4'-Methoxy-[1,1'-biphenyl]-2-yl\}-3,5-dimethyl-1H-pyrazole (12b)

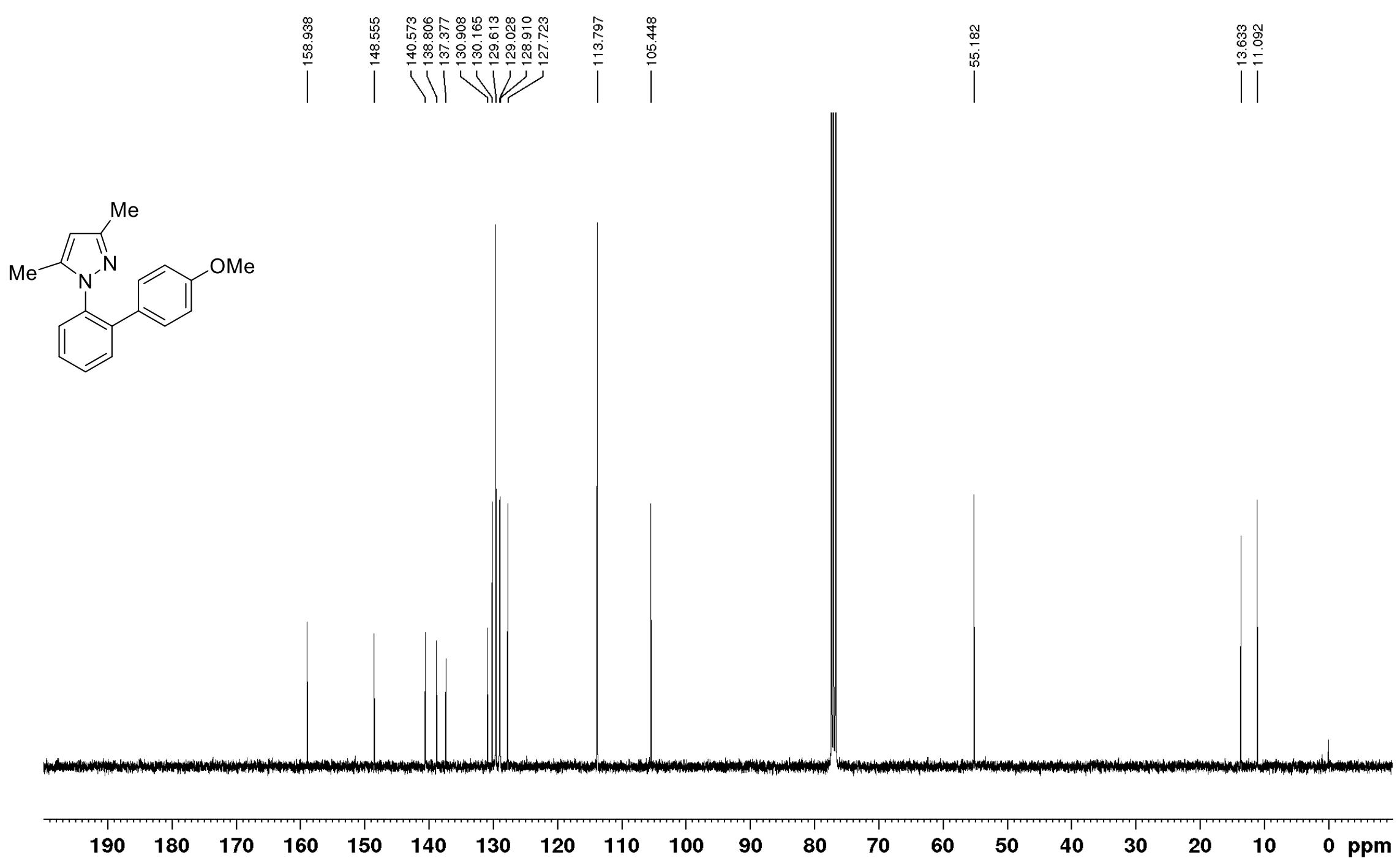


${ }^{1} \mathrm{H}$ NMR $\left(\mathrm{CDCl}_{3}, 400 \mathrm{MHz}\right)$ of 1-(2-benzylphenyl)-3,5-dimethyl-1H-pyrazole (13b)
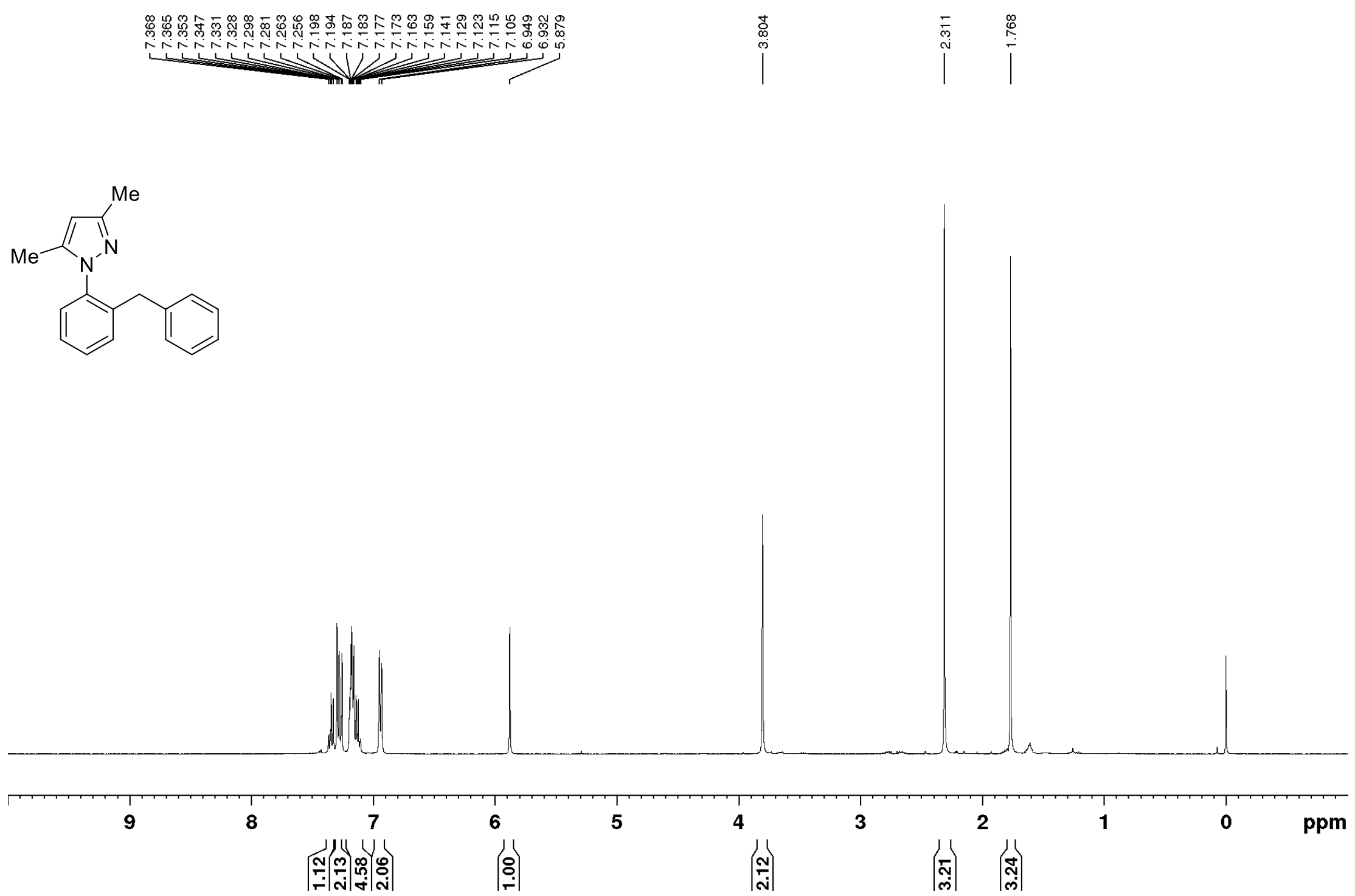
${ }^{13} \mathrm{C} \mathrm{NMR}\left(\mathrm{CDCl}_{3}, 100 \mathrm{MHz}\right)$ of 1-(2-benzylphenyl)-3,5-dimethyl-1H-pyrazole (13b)

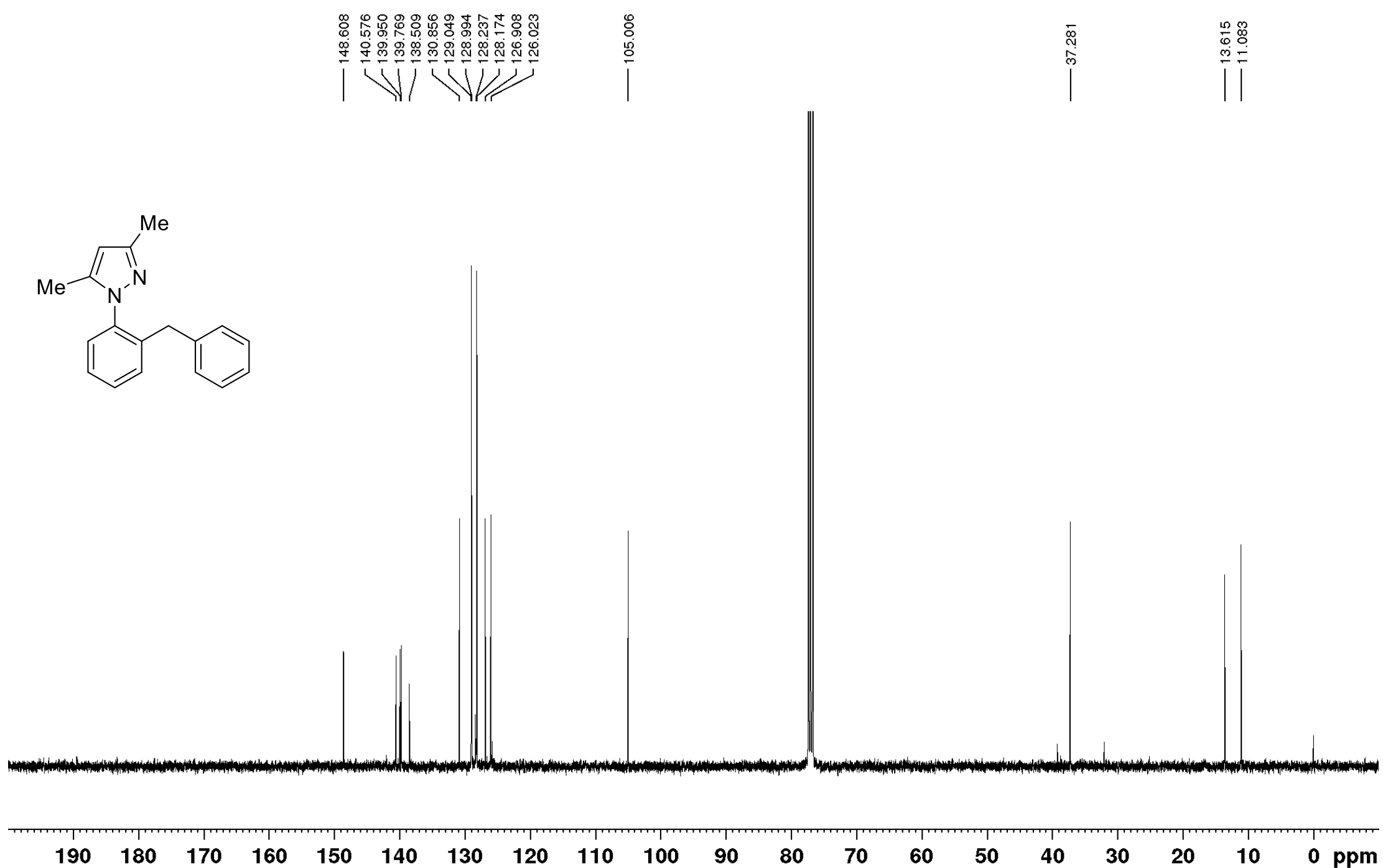


${ }^{1} \mathrm{H}$ NMR $\left(\mathrm{CDCl}_{3}, 400 \mathrm{MHz}\right)$ of 2,2'-Bis(3,5-dimethyl-1H-pyrazol-1-yl)-1,1'-biphenyl (14b)
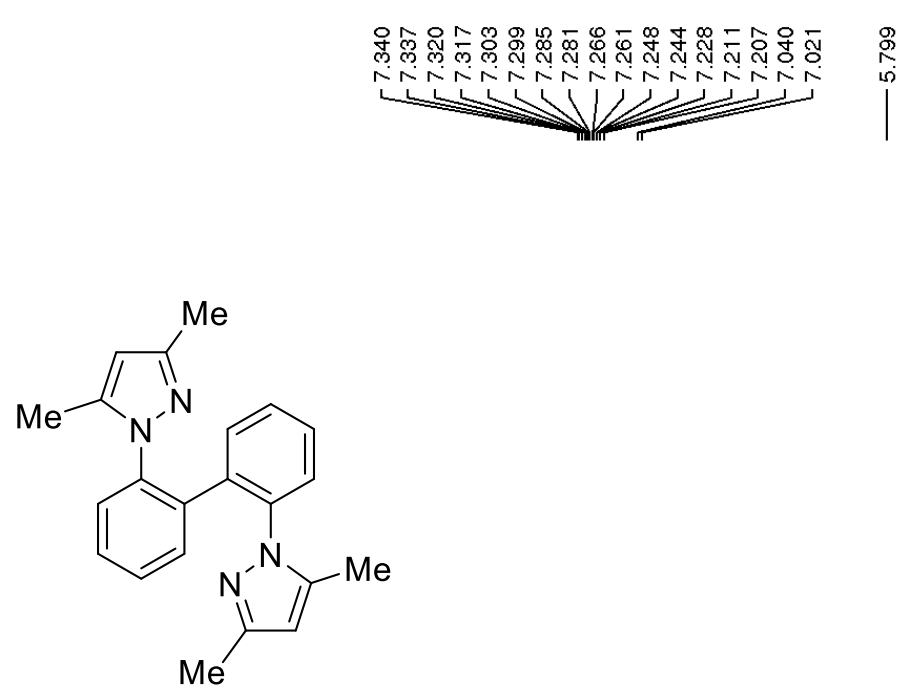

$\mathrm{Me}$

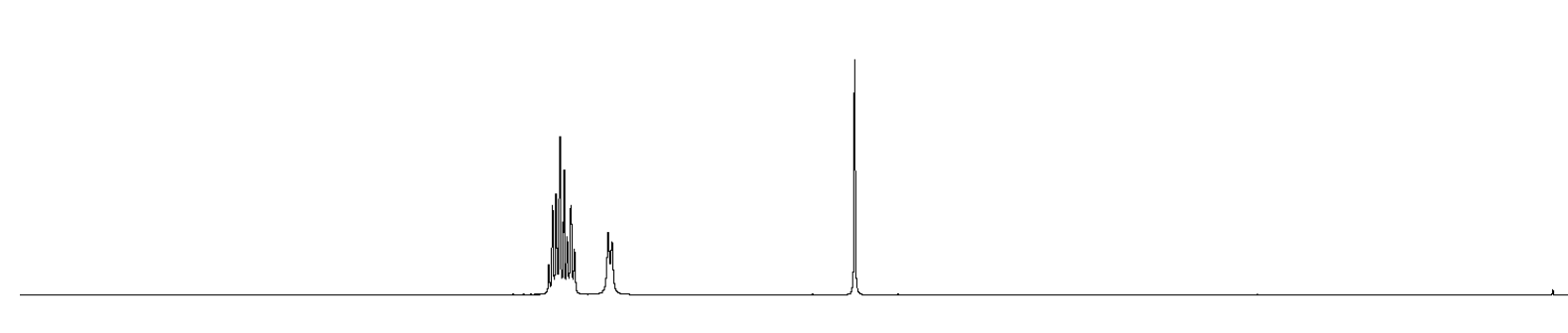

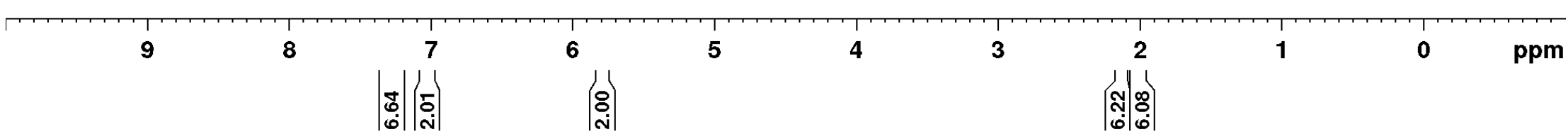


${ }^{13} \mathrm{C}$ NMR $\left(\mathrm{CDCl}_{3}, 100 \mathrm{MHz}\right)$ of 1-(2-Chlorophenyl)-3,5-dimethyl-1H-pyrazole (14b)

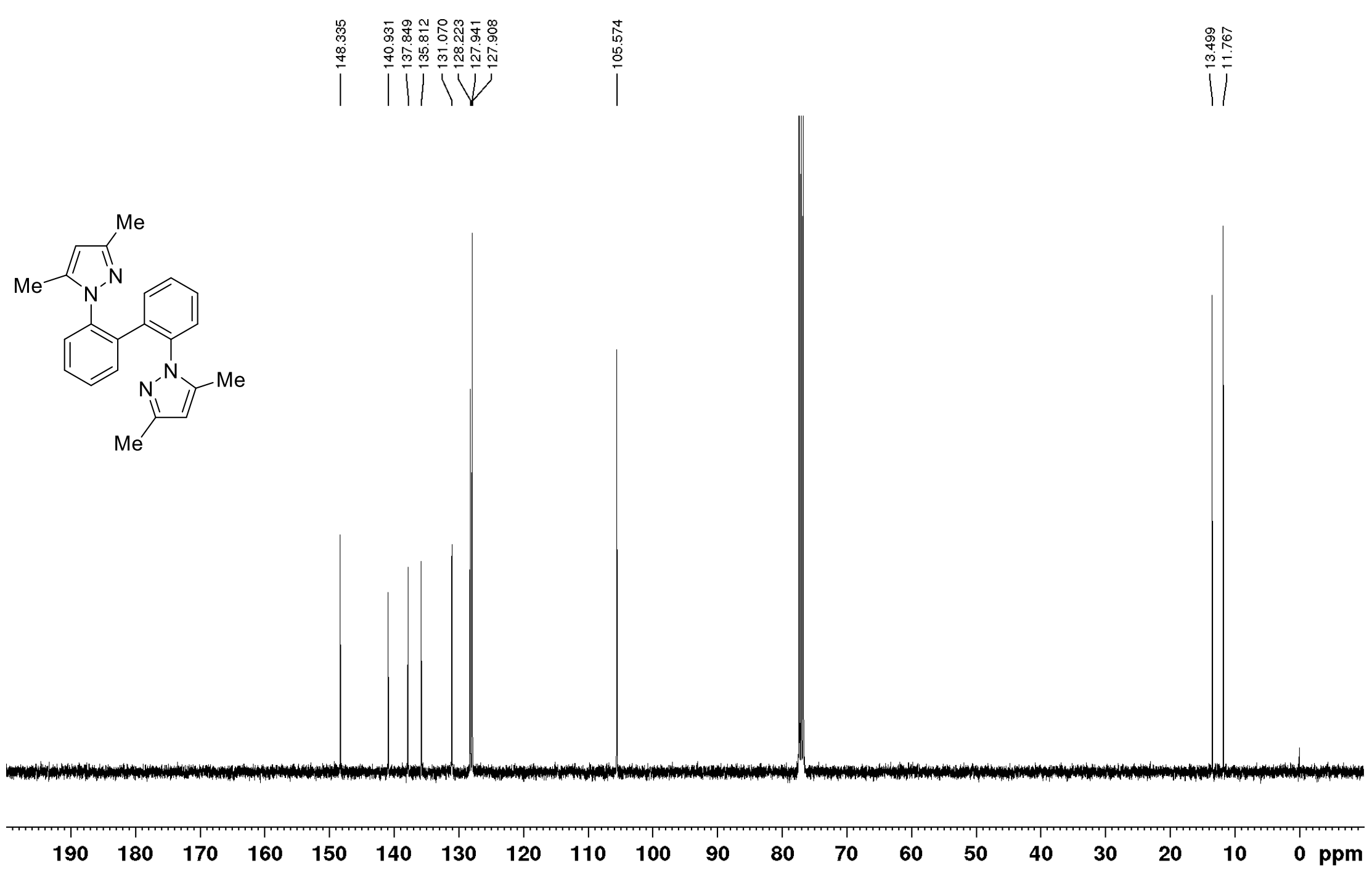


${ }^{1} \mathrm{H}$ NMR $\left(\mathrm{CDCl}_{3}, 400 \mathrm{MHz}\right)$ of 2-\{4,6-Diphenyl-3,5-dihydro-1H-cyclopenta[c]furan-5-yl $\}-N$-(4-methoxyphenyl)acetamide

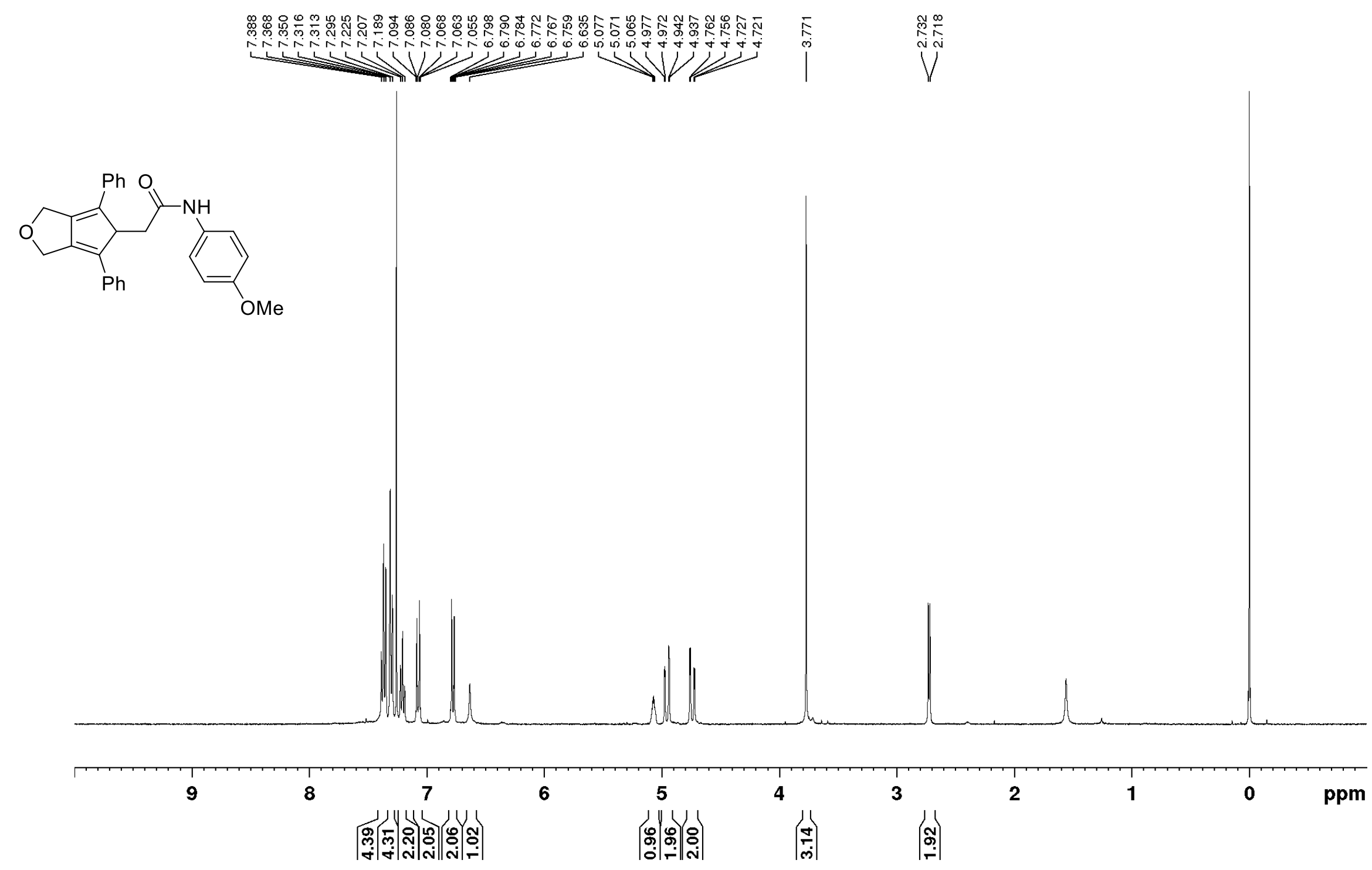


${ }^{13} \mathrm{C}$ NMR $\left(\mathrm{CDCl}_{3}, 100 \mathrm{MHz}\right)$ of 2-\{4,6-Diphenyl-3,5-dihydro-1H-cyclopenta[c]furan-5-yl $\}-N$-(4-methoxyphenyl)acetamide

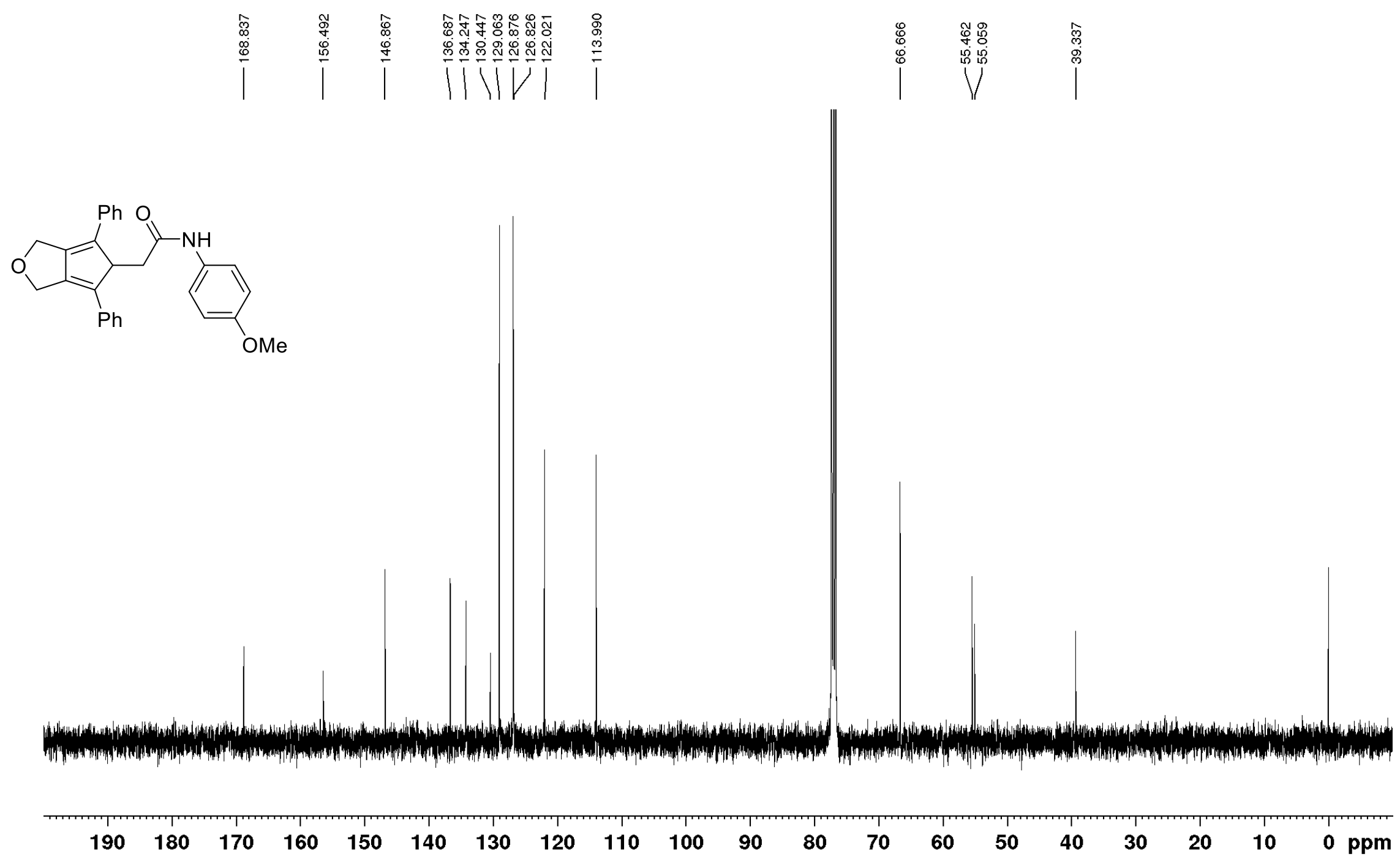


${ }^{1} \mathrm{H}$ NMR $\left(\mathrm{CDCl}_{3}, 400 \mathrm{MHz}\right)$ of $\left[\mathrm{Cp}^{\mathrm{A}} \mathrm{Rh}^{\mathrm{R}}(\mathrm{cod})\right](18)$

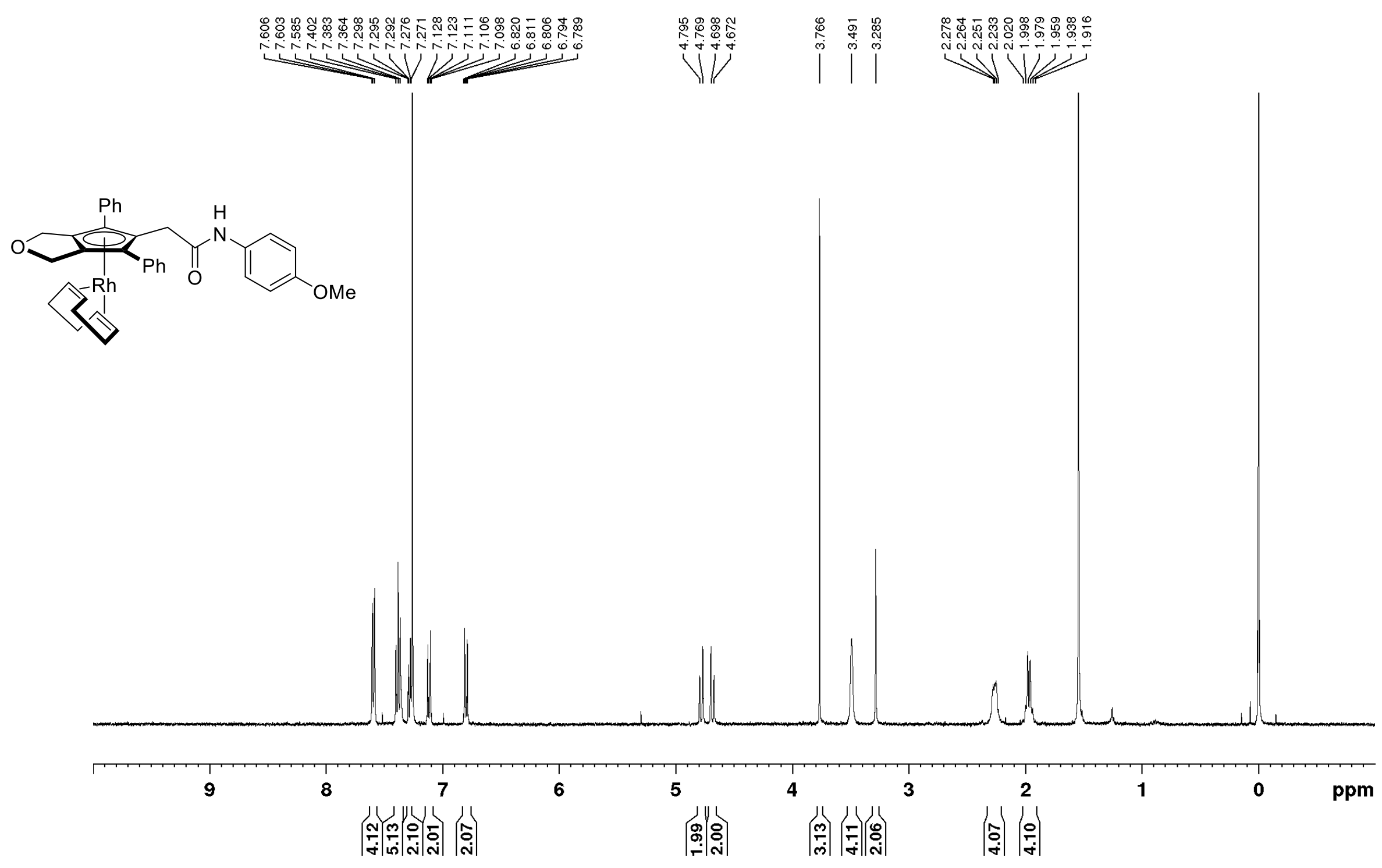


${ }^{13} \mathrm{C} \mathrm{NMR}\left(\mathrm{CDCl}_{3}, 100 \mathrm{MHz}\right)$ of $\left[\mathrm{Cp}^{\mathrm{A} 1} \mathrm{Rh}(\operatorname{cod})\right](18)$

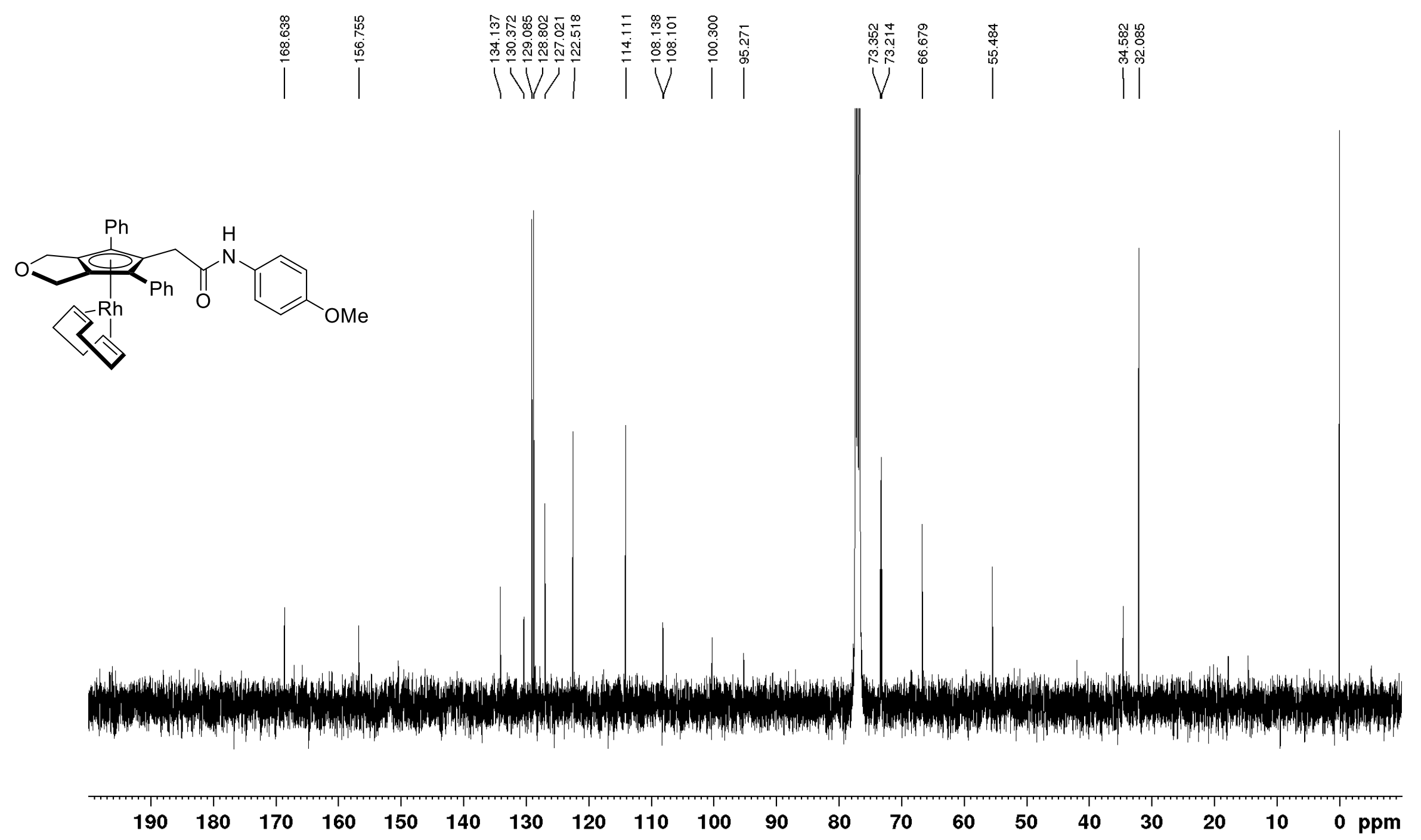


${ }^{1} \mathrm{H}$ NMR $\left(\mathrm{CDCl}_{3}, 400 \mathrm{MHz}\right)$ of [2-(1H-Pyrazol-1-yl)phenyl](pentamethylcyclopentadienyl)rhodium(III) acetate (A1)

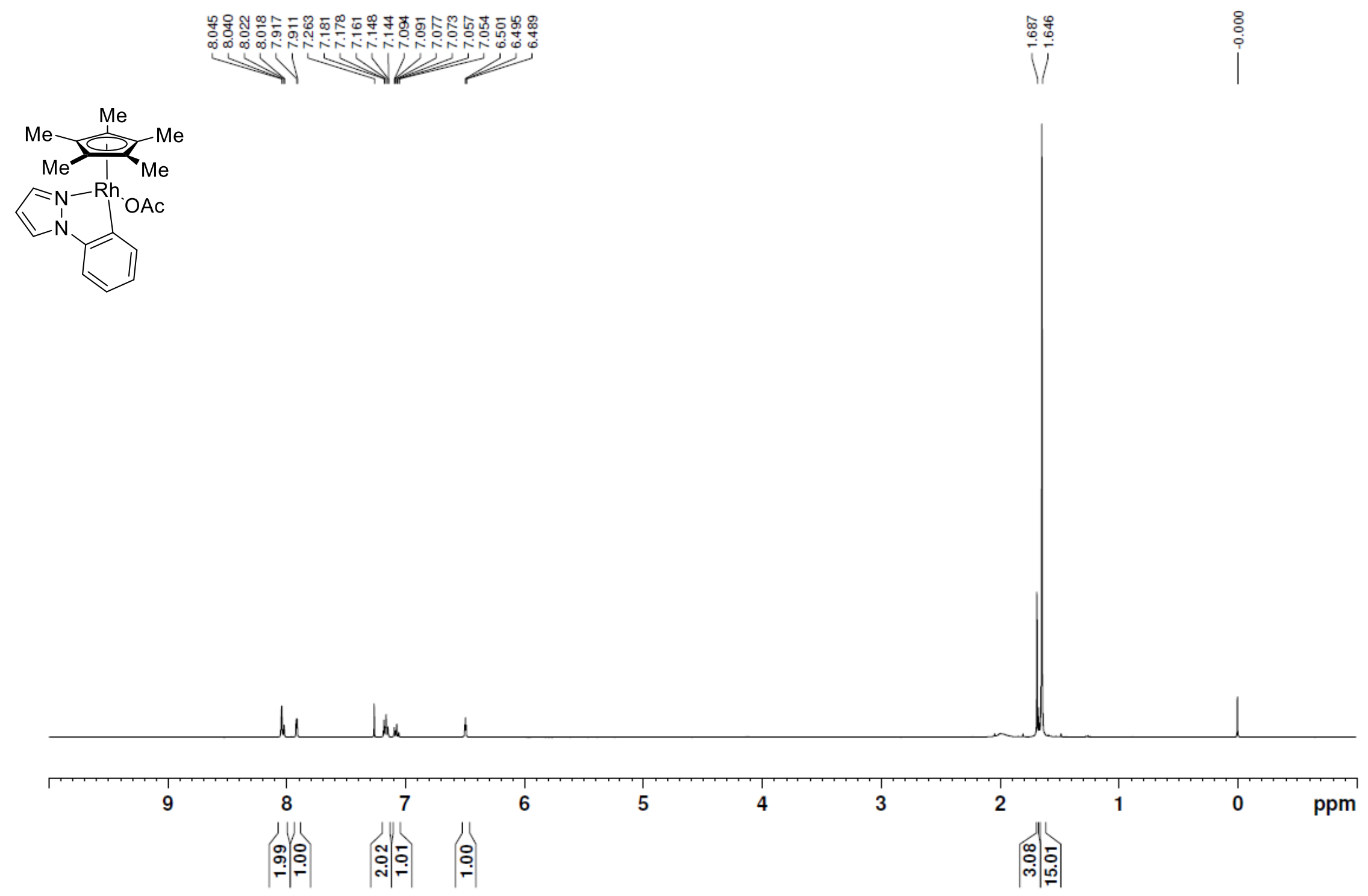


${ }^{13} \mathrm{C} \mathrm{NMR}\left(\mathrm{CDCl}_{3}, 100 \mathrm{MHz}\right)$ of [2-(1H-Pyrazol-1-yl)phenyl](pentamethylcyclopentadienyl)rhodium(III) acetate (A1)

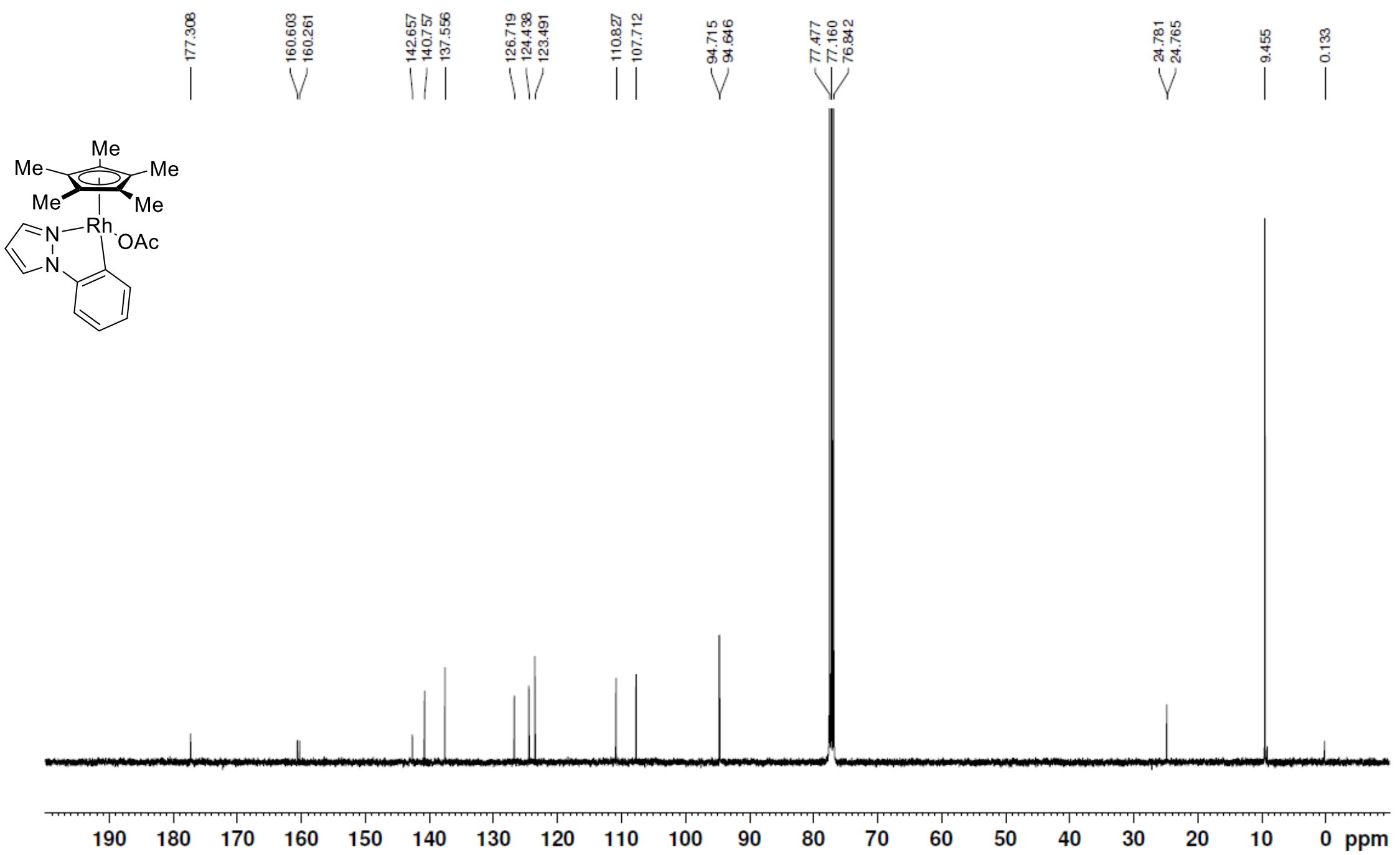


${ }^{1} \mathrm{H}$ NMR ( $\left.\mathrm{CDCl}_{3}, 400 \mathrm{MHz}\right)$ of [2-(2-pyridinyl)phenyl](pentamethylcyclopentadienyl)rhodium(III) acetate (A3)

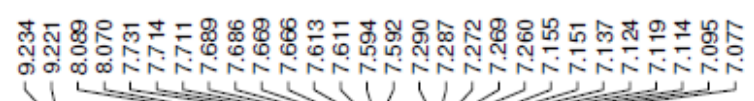
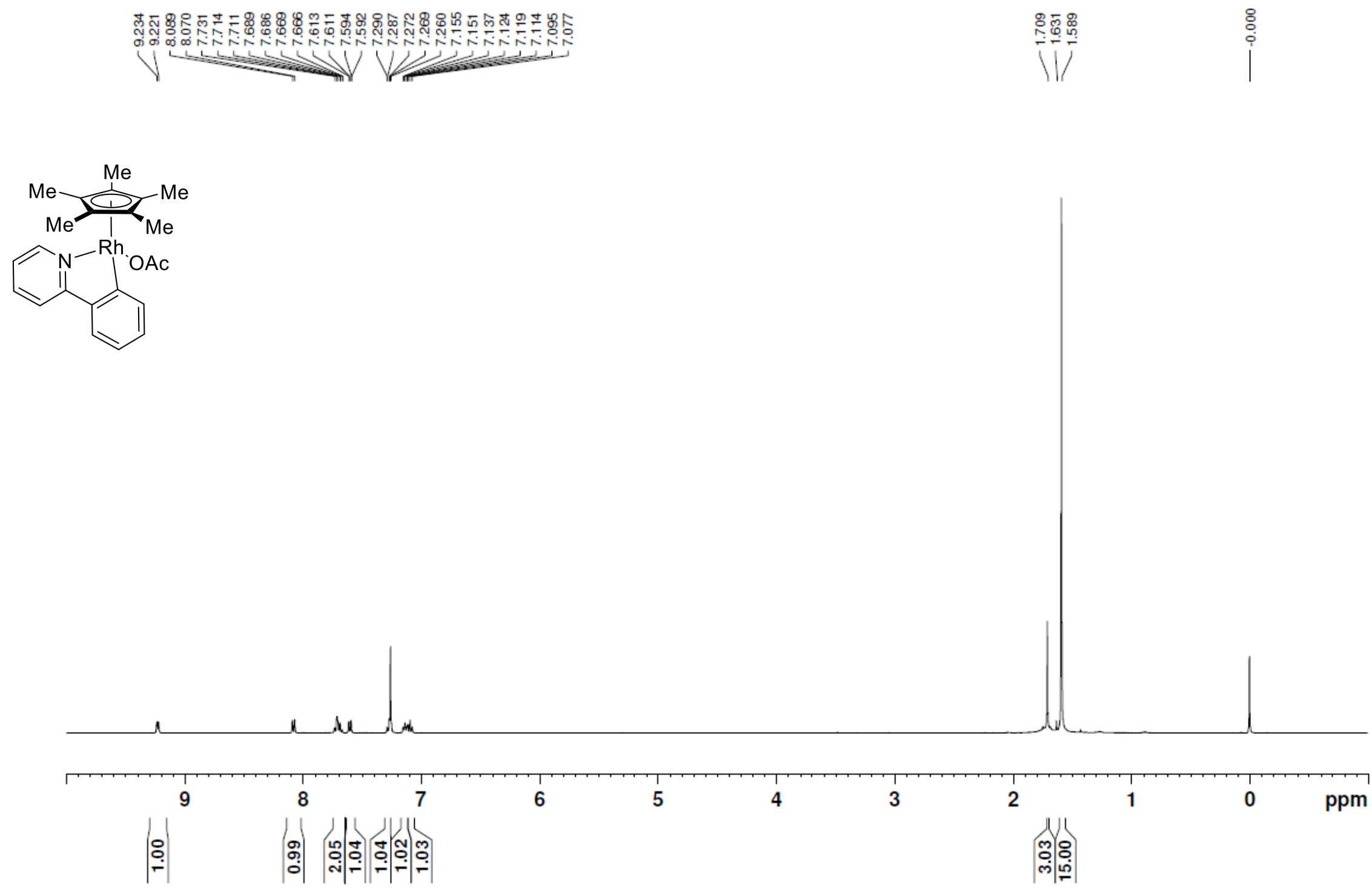


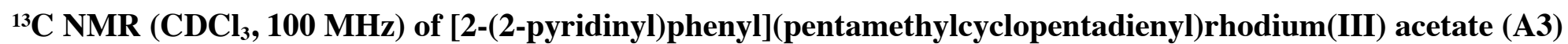

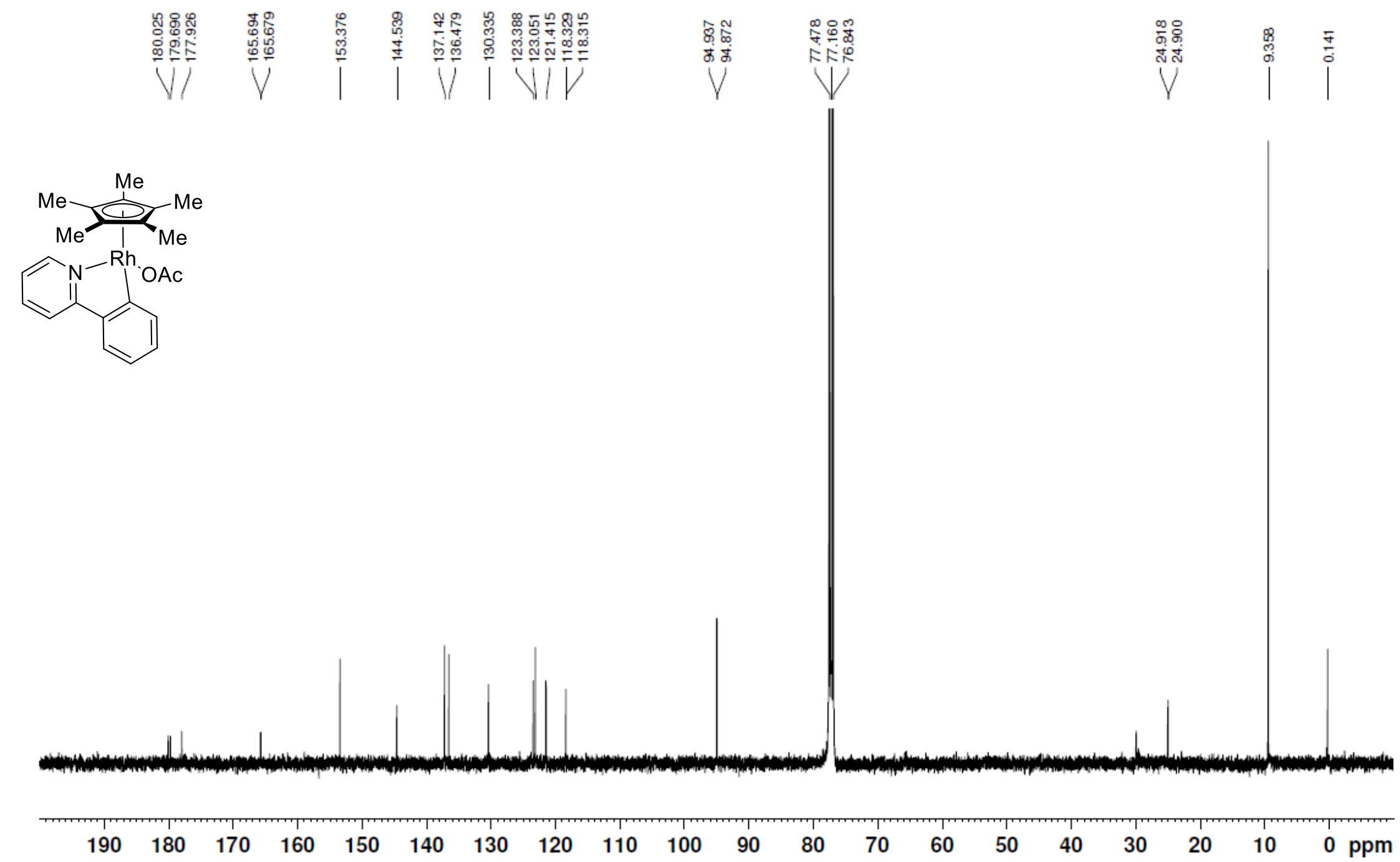

